\title{
Belief Change without Compactness
}

\author{
Jandson Santos Ribeiro Santos \\ A Thesis SUBMiTTED \\ TO THE \\ Institute of Mathematics And Statistics \\ OF \\ University of SÃo PAUlO \\ IN FULFILMENT OF THE REQUIREMENTS \\ FOR THE DEGREE \\ OF \\ DOCTOR OF SCIENCE
}

\author{
Supervisor: Prof. Dr. Renata Wassermann \\ Co-supervisor: Prof. Dr. Abhaya Nayak
}

The author was supported by a scholarship from CAPES and a scholarship from Macquarie University for visiting Macquarie University.

São Paulo, September 2019 


\section{Belief Change without Compactness}

This is the original version of the Thesis written by the candidate Jandson Santos Ribeiro Santos and submitted to the examination committee. This thesis was submitted to Macquarie University and University of São Paulo (USP) in accordance with the Cotutelle agreement dated 6th June 2017. 


\section{Acknowledgements}

I thank my supervisors A/Prof. Renata Wassermann and A/Prof. Abhaya Nayak. I also would like to thank my friends, and the administrative staff at both University of São Paulo (USP) and Macquarie University. I acknowledge the financial support of Macquarie University and the CAPES Foundation (Brazil). 


\section{Resumo}

Um dos principais objetivos da Inteligência Artificial (IA) é desenvolver agentes capazes de tomar decisões racionais de forma autônomo. Para isso, é essencial criar mecanismos para a representação do conhecimento que um agente tem sobre o mundo. Tais mecanismos devem possibilitar ao agente raciocinar sobre o conhecimento adquirido. No entanto, o conhecimento de um agente não é estático - ele é atualizado à medida que o agente adquire novas informações. Um dos grandes desafios que envolvem a representação do conhecimento é como um agente deve gerir seu corpo de conhecimento em resposta a novas informações que adquire: problema conhecido como Mudança de Crença.

As abordagens padrão de Mudança de Crença apresentam duas formas: um conjunto de postulados da racionalidade que prescrevem comportamentos epistêmicos para um agente; e uma coleção de construções ou funções para executar tais mudanças racionais. Os dois principais paradigmas da Mudança de Crenças são o paradigma AGM (para mudança de crença em um ambiente estático) e o paradigma KM (para mudança de crença em um ambiente dinâmico). Ambos os paradigmas fazem fortes suposições sobre a lógica subjacente usada para expressar as crenças de um agente, como por exemplo a Supraclassicalidade e a Compacidade. Depender dessas suposições, no entanto, é bastante restritivo, pois muitas lógicas importantes para aplicações de IA e de Ciência da Computação não as possuem. Esta tese tem como foco estender a Mudança de Crenças para o domínio das lógicas sem compacidade.

Um dos efeitos colaterais em dispensar a Compacidade é que as construções padrões dos paradigmas da AGM e da KM não se conectam mais com os respectivos postulados de racionalidade. Neste trabalho, identificamos as razões por trás dessa falha. Por sua vez, isso nos ajuda a identificar algumas condições mínimas sob as quais a existência de operações racionais de mudança de crença nos paradigmas AGM e KM é garantida. Posteriormente, fornecemos operações de Mudança de Crença para ambos paradigmas AGM e KM sem a suposição da Compacidade.A principal diferença entre a nossa abordagem e as abordagens padrões está na forma como a preferência epistêmica de um agente é representada: em vez de Remainders e Sistemas de Esferas de Grove, consideramos teorias completas e relações parciais genuínas sobre mundos.

Além disso, também consideramos a conexão entre a revisão de crenças AGM e os Sistemas Lógicos Não-Monotônico (SLNM), geralmente vistos como dois lados da mesma moeda. Demonstramos que a ponte entre a revisão de crenças e SLNMs se rompe na ausência da Compacidade. Em seguida, identificamos a causa do problema, e apresentamos um novo sistema não-monotônico que se conecta adequadamente aos postulados de revisão AGM, mesmo na ausência de compacidade. Significativamente, essa conexão com o paradigma AGM é independente de quaisquer construções específicas (como sistemas de esferas) e é diretamente estabelecida entre os postulados AGM e os axiomas do sistema não-monotônico proposto.

Keywords: Revisão de Crenças, Atualização de Crenças, Compacidade, Sistemas Não Monotônicos. 


\section{Abstract}

One of the main goals of Artificial Intelligence (AI) is to build rational agents that are capable of taking rational decisions autonomously. For this, it is essential to devise mechanisms to properly represent knowledge, and reason about the knowledge that an agent has about the world. However, an agent's knowledge is not static - it gets updated as the agent acquires new information. One of the big challenges involving knowledge representation is how an agent ought to change its own knowledge and beliefs in response to any new information it acquires. This, in short, is the problem of belief change.

Standard approaches of Belief Change come in two flavours: a set of rationality postulates that prescribes epistemic behaviours for an agent, and a collection of constructions, or functions, to perform such rational changes. The two foremost paradigms of Belief Change are the AGM paradigm (for belief change in a static environment) and the KM paradigm (for belief change in a dynamic environment). Both these paradigms make strong assumptions about the underlying logic used to express an agent beliefs, such as Supraclassicality and Compactness. Relying on these assumptions, however, is rather restrictive, since many logics that are important for both AI and Computer Science applications do not have them. This thesis focuses on extending Belief Change to the realm of non-compact logics.

One of the side effects of dispensing with compactness is that standard constructions of both the AGM and the KM paradigms no longer nicely connect with the respective rationality postulates. In this work, I identify the reasons behind this breakdown. This in turn helps us identify some minimal conditions under which the existence of rational AGM and KM belief change operations is guaranteed. Subsequently we provide constructive accounts of AGM- and KM-rational belief change operations without the compactness assumption, and we offer full accounts of belief change for both the paradigms. The main difference of our approach from the standard ones relies on the way epistemic preference of an agent is represented: instead of remainders and Grove's Systems of Spheres, we consider maximal complete theories and genuine partial relations over worlds.

Furthermore, we also consider the connection between AGM revision and non-monotonic reasoning (NMR) systems, often viewed to be two sides of the same coin. We demonstrate that the bridge between belief revision and NMR breaks down in the absence of compactness. We then identify the basis of this breakdown, and present a new non-monotonic system that appropriately connects with the AGM revision postulates even in absence of compactness. Significantly, this connection with the AGM paradigm is independent of any specific constructions (such as systems of spheres), and is directly established between the AGM postulates and the axioms of the proposed non-monotonic system.

Keywords: Belief Revision, Belief Update, Compactness, Non-Monotonic reasoning. 


\section{Contents}

$\begin{array}{ll}\text { List of Figures } & \text { ix }\end{array}$

List of Tables $\quad$ xi

1 Introduction $\quad 1$

1.1 Belief Change minus Compactness . . . . . . . . . . . . . . . . . . 3

1.2 Non-Monotonic Reasoning minus Compactness . . . . . . . . . . . . . . . 3

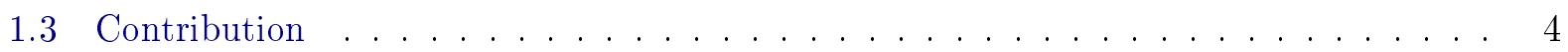

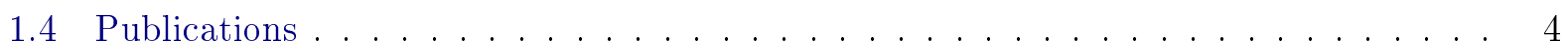

1.5 Related Works . . . . . . . . . . . . . . . . . . 5

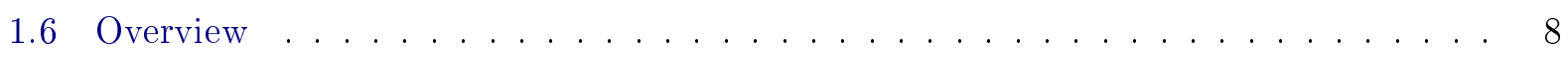

1.7 Notation . . . . . . . . . . . . . . . . . . . . . 8

2 Belief Systems and the AGM Paradigm $\quad 11$

2.1 Epistemic states, inputs and changes . . . . . . . . . . . . . . . . 11

2.2 AGM Paradigm . . . . . . . . . . . . . . . . . . . . . . . . . . . . . . . . . . . . . . . . . . . . . . . . .

2.2 .1 Contraction . . . . . . . . . . . . . . . . . . 13

2.2 .2 Revision . . . . . . . . . . . . . . . . . . . . 18

2.3 AGM Compliance . . . . . . . . . . . . . . . . . . . . 21

2.3.1 Decomposability and AGM Compliance . . . . . . . . . . . . . 21

3 Dispensing with Compactness in the AGM Paradigm 25

3.1 AGM compliance without compactness . . . . . . . . . . . . . 26

3.2 Construction: AGM Contraction Basic Rationality . . . . . . . . . . . . . . . . . 30

3.3 Construction: Full AGM Contraction Rationality . . . . . . . . . . . . . . . 35

3.4 AGM Revision Rationality . . . . . . . . . . . . . . . . . . . . . . 42

3.4.1 Exhaustive Revision Function: AGM basic rationality . . . . . . . . . . . 43

3.4.2 Constellar Revision Function: Full AGM Revision Rationality . . . . . . . . . 45

3.5 Levi Identity and Harper Identities . . . . . . . . . . . . . . . . . . 51

4 KM Update and Non-Finitary Languages without Compactness $\quad 53$

4.1 KM Update . . . . . . . . . . . . . . . . . . . . . . . 54

4.2 The Problem of Update in Non-finitary logics . . . . . . . . . . . . . . 56

4.3 Reconstructing Rational Update Functions . . . . . . . . . . . . . . . . . . . . . . . . . . . . 57

4.3 .1 KM Basic Rationality . . . . . . . . . . . . . . . . . . . . 57

4.3.2 Revisiting the Supplementary Postulates . . . . . . . . . . . . . 60 
4.4 Looking back at CUP functions . . . . . . . . . . . . . . . . . 66

4.5 Are total relations really needed $\ldots \ldots \ldots \ldots \ldots \ldots$

4.6 Discussion . . . . . . . . . . . . . . . . . . . . . . . 69

$5 \quad$ AGM Belief Revision and Non-Monotonic Systems $\quad 71$

5.1 Non-monotonic reasoning . . . . . . . . . . . . . . . . . 73

5.2 From ${ }^{*}$ to $\sim$ without Compactness . . . . . . . . . . . . . . . 74

5.3 From $\sim \sim$ to ${ }^{*}$ without Compactness . . . . . . . . . . . . . . . 75

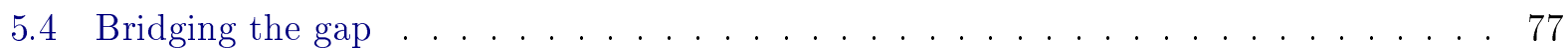

5.4 .1 Capturing Supplementary Postulates . . . . . . . . . . . . . . . 80

5.5 Summary and the Highlights . . . . . . . . . . . . . . . . . . . 81

6 Concluding Remarks $\quad 83$

6.1 Main Contributions . . . . . . . . . . . . . . . . . . . . . . 83

6.2 Discussion and Future Work . . . . . . . . . . . . . . . . . . . 85

$\begin{array}{ll}\text { A Proofs for Chapter 3, Section } 3.1 \text { to } 3.3 & 91\end{array}$

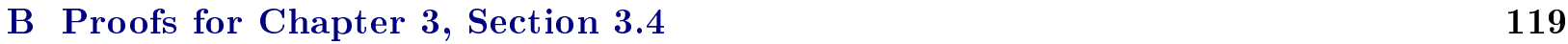

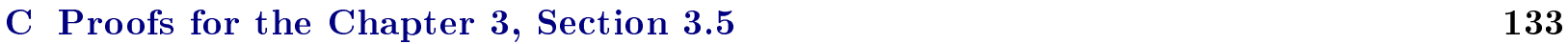

D Proofs for Chapter $4 \quad 139$

$\begin{array}{ll}\text { E Proofs for Chapter } 5 & 157\end{array}$

$\begin{array}{ll}\text { Bibliography } & 165\end{array}$ 


\section{List of Figures}

2.1 An epistemic entrenchment relation. . . . . . . . . . . . . . . . 17

2.2 A System of Spheres. . . . . . . . . . . . . . . . . . . . . . . . 19

2.3 A system of Spheres for the function of Example $5 \ldots \ldots \ldots \ldots \ldots$

3.1 A schemata to construct a relative complement $K^{\prime}$ for a theory $K$ and $A$. . . . . . 26

3.2 A schema to find a theory in a contraction operation. . . . . . . . . . . . 33

3.3 Mirroring. A is preferred to $\mathrm{C}$, hence to D. . . . . . . . . . . . . . . 35

3.4 A depiction of Example 8. . . . . . . . . . . . . . . . . 37

3.5 Three relations: $<_{\varphi},<_{\psi}$ and $<_{\varphi \wedge \psi} \ldots \ldots \ldots \ldots \ldots \ldots \ldots \ldots \ldots$

3.6 A sovereign relation for Example 10 . . . . . . . . . . . . . . . . 47

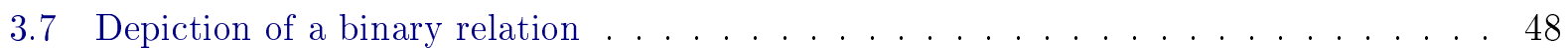

3.8 A binary relation $<$ for the $K$-royal coalition function in Example 11 . . . . . . . . 50

4.1 A preorder for the function in Example 13. . . . . . . . . . . . . 56

4.2 A Sovereign Relation. . . . . . . . . . . . . . . . . . . . . . 60 


\section{List of Tables}

2.1 Some logics and their relation with AGM and relevance-compliance . . . . . . . . . 23 


\title{
Chapter 1
}

\section{Introduction}

\begin{abstract}
[...] Alice ran with all speed back to the little door: but, alas! the little door was shut again, and the little golden key was lying on the glass table as before, 'and things are worse than ever,' thought the poor child - Lewis Carroll, Alice in Wonderland.
\end{abstract}

Representing knowledge and reasoning about that knowledge are fundamental to Artificial Intelligence (AI). In devising intelligent and autonomous systems (or agents), the knowledge of an agent needs to be represented in a way that the agent can manipulate it in order to take decisions.

Knowledge, however, is not static and keeps changing. In the quote of Lewis Caroll above, for instance, Alice had to change her beliefs about the little room she has left behind just after passing through the door. There are many other examples in which we, humans, have to change our beliefs. For instance, until the 15th century, people used to believe in the geocentrism, which puts Earth as the center of the Solar System. This changed, however, after the 16th century with the heliocentrism, which puts the sun at the center instead. Earth was taken to be flat until it was found to be round.

Since we, humans, often change our knowledge, it is expected that artificial intelligent systems also need to change their knowledge from time to time. This is essentially true when an agent needs to interact with the environment and acquire new information from it, and therefore deal with the dynamics involved with its tasks that might affect its beliefs. These changes might come from its own actions, from an external source, or even from a conversation involving two, or more, rational agents. Regardless of the way that an agent acquires new information, the fact is that it is necessary to manage its body of knowledge and deal with the changes accordingly.

The field in AI that studies the dynamics involved in keeping an agent body of knowledge up to date is known as Belief Change (Gärdenfors, 1988). The two most influential theories of Belief Change are the AGM paradigm, initially proposed in (Alchourrón et al., 1985) and further developed in works such as (Hansson, 1999; Rott, 1992); and the KM paradigm (Katsuno and Mendelzon, 2003). Both approaches conceptualise the notion of rational change motivated by the idea that the changes involved should be minimised. This minimality principle is formalised by rationality postulates which prescribe good behaviours for belief change operators. Besides the postulates, both AGM and KM paradigms present classes of belief change operators that behave accordingly to their rationality postulates.

The beliefs of an agent are expressed as formulae specified in the classical propositional logic. Relying solely on proposition logic to represent knowledge, however, is too restrictive. There are 
many other kind of knowledge that needs to be expressed in AI systems. For instance, conceptual knowledge which can be expressed via Description Logics (Baader et al., 2003), Horn Logic (Horn, 1951) which are used for rule-based systems, and a variety of Modal Logics, such as Temporal Logics (Gabbay et al., 1994), which are widely used in planning. To this end, considering belief change in the context of non-classical logics is essential to develop more realistic intelligent systems.

This thesis is an effort to extend belief change to a bigger class of non-classical logics. Specifically, we intend to make belief change more independent of assumptions about the underlying logic.

Extending belief change beyond the scope of propositional logic, however, is not a trivial task. There are two main reasons for the hurdle: whereas in some logics, the standard belief change operators do not satisfy the rationality postulates, in other logics it is not even possible to define belief change operators that behave accordingly to the rationality postulates. The latter is the case of logics such as Horn Logic, Intuitionist Logics and classes of Descriptions Logics (Ribeiro, 2013). The issue of belief change for non-classical logics has also been examined by many such as (Restall and Slaney, 1995) and (Wassermann, 2011).

One of the most relevant kind of knowledge used in AI is temporal knowledge. Among the many fields in AI in which Temporal Logics are used to represent knowledge, we can cite planning, reasoning about actions, and sensing actions. In all these applications, temporal logics are used to represent the knowledge an agent has about the environment it is in, and it has also to deal with changes. Although, one can represent such kind of knowledge, dealing with the dynamics of beliefs is still a challenge, and approaches to deal with changes is usually ad-hoc.

Given the relevance of temporal logics in AI, understanding the dynamics involved in the management of knowledge in these logics will allow the construction of enhanced and more realistic intelligent systems. To this end, as for classical logics, a general framework capable of explaining and guiding belief change in these logics would be ideal. This, however, is not as easy as it may seem. The standard approaches of Belief change make some strong assumptions about the background logic used to represent an agent's knowledge in terms of what properties they satisfy. Such assumptions may excessively limit when and how belief change can be performed. One of these assumptions is that the logic in question satisfies compactness, which states that if a formula $\varphi$ is entailed by a set of formulae $K$, then $\varphi$ must also be entailed by a finite subset of $K$.

Example of non-compact logics include Hybrid logics, such as PDL which are used to represent the dynamics of computational systems; and more expressive temporal logics, such as CTL and LTL, which are among the most widely used logics in AI. As investigated by (Ribeiro et al., 2013), compactness has an important role in belief change theory, and dispensing with it has serious consequences. For instance, in the AGM paradigm the connection between the standard constructions of belief change and the postulates is lost. Even worse, (Guerra, 2016) has shown that it is not possible to define such functions in logics without compactness. Regardless of how we intend to extend belief change to temporal logics, it is clear we have to deal with the problem of discarding compactness. This is the main purpose of this thesis: how to extend belief change for non-compact logics. Addressing compactness will not only allow us to apply belief change for a huge class of logics, but also to understand why the traditional mechanisms of belief change depend so strongly on this property and, therefore, how to understand better belief change itself. In addition, it will provide us to assume very little about the underlying logic, and look at belief change more freely, 
focusing on the aspect of rationality change rather than on properties from the underlying logic.

\subsection{Belief Change minus Compactness}

This thesis is an effort to extend belief change for logics without compactness. There are a few questions that one may naturally raise:

1. Suppose we construct a belief change function analogous to the way it is constructed in the AGM tradition, such as Partial Meet Contraction, Transitively Relational Partial Meet Contraction, assuming a non-compact logic as the background logic. Will the standard AGM postulates of contraction still be appropriate? If not, what alternative postulates will characterise such belief contraction functions?

(a) The same issue, but with respect to Belief update in the KM tradition, mutatis mutandis.

2. Suppose we agree upon a set of belief change postulates that are arguably rational in the context of a background logic that is non-compact. Can we construct a belief change function that satisfies some, or all, of those postulates? If so, how?

3. Since AGM (respectively, KM) Postulates have survived the trial of time, we may assume that they are appropriate even if the background logic is non-compact. What other assumptions must we make about the background logic? Can we construct a belief change function that will satisfy them, and if so, how?

Addressing all these issues is perhaps too ambitious, so I focus on the thirs issue in this thesis. The first question to pose if it is even possible to define belief change functions according to AGM and KM paradigms rationality postulates. Toward this end, we identify the properties a logic must posses in order to guarantee the existence of such functions, without depending on compactness. We do this for both AGM paradigm and KM paradigm. For the latter, we also identify the rationality postulates that emerge from the standard constructions of the KM paradigm in the absence of compactness.

\subsection{Non-Monotonic Reasoning minus Compactness}

Classical logics are monotonic: the acquisition of new information does not expel the old information. There are scenarios, however, in which monotonicity needs to be abandoned, as in the following example.

Example 1. John works on the city centre and goes to his office by train. He takes a train at 11:00 AM every morning from Monday to Friday, and he always reaches his office at 11:40 AM. John has a meeting on Wednesday at 11:45 AM. He knows, therefore, that if he takes the train at 11:00 $A M$ as usual he will arrive in time for his meeting. On Wednesday morning, however, arriving at the train station, John is warned that the train of 11:00 AM will be delayed for 15 minutes, due to weather issues. John does not believe any more that he will arrive in time for his meeting. 
In the example above, after knowing that the train will be delayed, John cannot sustain his belief any more that he will arrive in time for his meeting. This process of deactivating old beliefs in the presence of new ones is no-monotonic. From this perspective, non-monotonicity and belief change seem very similar. Indeed, it has been argued that both non-monotonic reasoning and belief change are two sides of the same phenomenon. This is essentially true when the background logic is classical. Indeed, the non-monotonic system of expectation (Gärdenfors and Makinson, 1994) and belief revision (one of the AGM forms of belief change) are inter-definable.

As the main purpose of this thesis is to extend belief change for non-compact logics, a natural question to pose is: what happens to the connection between non-monotonic reasoning and belief revision when compactness is discarded? Towards this end, we investigate whether or not the interconnection between belief revision and non-monotonic logics based on expectation still remains when compactness is dropped. We will show that though belief revision can still be translated to non-monotonic reasoning, the converse is not true. We present, then, a new non-monotonic system that reconnects with AGM revision independent of compactness.

\subsection{Contribution}

In summary, the main contributions of this work are:

1. Expansion of AGM and KM paradigms to the realm of non-compact logics.

2. Characterisation of classes of functions that are (fully) AGM rational for both belief contraction and revision.

3. Demonstrating that the inter-correspondence between contraction functions and revision functions via Levi and Harper identities are not affected when dispensing with compactness.

4. Identifying a complete set of rationality postulates that emerges from the standard KM update functions, when compactness is abandoned.

5. New classes of (fully) KM rational update functions.

6. Two main results concerning the connection between AGM revision and non-compact logics:

(a) Proof that the connection between AGM revision and the non-monotonic system based on exception breaks down in the absence of compactness;

(b) A new non-monotonic system that connects with AGM revision independent of compactness.

\subsection{Publications}

1. Belief Update without Compactness in Non-finitary Languages. IJCAI-2019: 1858-1864, with Abhaya Nayak and Renata Wassermann.

2. Belief Change and Non-monotonic Reasoning sans Compactness. AAAI 19: 3019-3026, with Abhaya Nayak and Renata Wassermann. 
3. Towards Belief Contraction without Compactness. KR 2018:287-296, with Abhaya Nayak and Renata Wassermann. This paper was awarded the Marco Cadolli prize of best student paper.

\subsection{Related Works}

Some previous works have considered belief change in the context of some specific non-classical logics. In this section we discuss these works and their limitations. We split these related works in two main groups: belief change as dynamic logics, and belief change in formal methods.

\section{Belief Change as Dynamic Logics}

Belief change studies the dynamics of beliefs and propose how to change an agent's beliefs in the presence of such dynamisms. It does not consider, however, the problem of reasoning about belief change. Consider for instance the following example adapted from (Van Ditmarsch et al., 2007).

Example 2. Alice and John are playing a card game. The goal is to find out the pair of cards that the opponent has after swapping some cards between them. The card stack has precisely 5 cards, numbered from 1 to 5. Each player takes 2 cards each from the pile. After seeing her cards, Alice realises that she has the cards with numbers 2 and 4. So she believes that John does not have these cards, but also believes that he does not know which cards she has, since there is yet one card on the pile. On the first round John and Alice swap one card. Alice got the card of number 1 and John the card of number 2. What Alice now knows about John's beliefs and what John knows about Alice's beliefs after such an action?

In the example above, conceptualisation of the change operators for Alice and John is far from obvious. One is concerned to reason about what each one of them know about each other's beliefs, and the effects that their actions have on each other's beliefs, in order to play the game. To make it clearer, what Alice and John know about each other's beliefs after swapping cards on the first round? And which information will John get if Alice reveals her card of number 4 in the next round? In this scenario, one is more interested in reasoning about the knowledge flow between the players rather than conceiving belief change operators for Alice and John.

Dynamic logics (Van Ditmarsch et al., 2007), such as Dynamic Epistemic Logic (DEL) and Dynamic Doxastic Logic (DDL) can be used for this purpose. These logics encapsulate belief change operators in the object-language as modal operators. In DDL, for instance, it is possible to write sentences such as $[* \varphi] B_{A} \psi$ which means that an agent $A$ believes in $\psi$ after revising its beliefs by $\varphi$. Writing sentences of such a kind allows to reason about the information flow of an agent and even reason about the belief dynamics involved in the interaction among two or more agents. The dynamic operators in DDL are designed to behave accordingly to the AGM rationality postulates. Precisely, a subset of it, since not all of these postulates can be captured in a modal logic due to Gärdenfors impossibility result (Gärdenfors, 1988). Dynamic logics, however, have a limitation. Consider that an agent's knowledge is grounded in propositional logics, and apart from higher levels of nesting modal operators, such as $B_{A} B_{C} \varphi$ which means agent $\mathrm{A}$ believes that agent $\mathrm{C}$ believes in $\varphi$, it is not possible to represent more expressive knowledge in these logics. 
Alchourrón (1995) proposed another way to represent a belief change operator into the language. His approach is based on defeasible condiational in which conditionals can behave in a non-monotonic way. For instance, in Example 1 taking the train at 11:00 AM (t) implies that John arrives at the office at 11:40 AM (m), this conditional can be expressed as $t \rightarrow m$. When the train gets delayed, however, John does not believe any more that he will arrive on time at the office. It is more likely to say that John would arrive at office on time, unless the train gets delayed. Alchourrón (1995) gives an interpretation of belief change over defeasible conditions in his DFT system. The main idea is that a sentence " $\varphi$ implies $\psi$ " means that $\psi$ is believed after revising the current belief state by a sentence $\varphi$. The system consists of a belief change operator $f$ inspired by the AGM belief revision postulates. It is assumed that an agent has a set of premisses $A$, and the sentence $f(p)$ combines $A$ and $p$. In the case that $p$ and $A$ are jointly inconsistent, $f$ behaves accordingly to some of the AGM revision postulates to deal with such inconsistencies. A defeasible conditional $f(p)>\varphi$ means that $\varphi$ is believed after revising the premisses $A$ in light of $p$. The implication $>$ is taken to be an alethic implication, interpreted in a modal system in order to avoid the problems of material implication. As DDL, DFT also vulnerable to the impossibility result of Gärdenfors.

Another problem that revolves around belief change its the iterative belief change (Darwiche and Pearl, 1997; Nayak, 1994; Nayak et al., 2003) that concerns the problem of how an agent changes its beliefs over time. In this case, the order in which an agent receives information and changes its beliefs is important. Bonanno (2009) has proposed to represent the iterative process of belief change in a temporal framework. His system consists of a doxastic operator $B_{A} \varphi$, meaning that an agent $\mathrm{A}$ Believes that $\varphi$ is true, and information operator which informs an agent about a new input, and a next-time operator to model the time feature of belief change. The main idea of Bonanno system is to capture in a natural way the temporal aspects of information flow aspect in iterative belief change .It behaves similarly to DDL, with the advantage of explicitly representing the iterative belief change naturally in the system via temporal operators.

Epistemic entrenchment and System of Spheres (Gärdenfors, 1988; Grove, 1988) are among the standard systems used to give semantics to AGM belief change. These systems are founded on total relations to represent the epistemic preferences an agent has about its own beliefs. The main idea is that when changing its beliefs an agent should maintain those most preferable ones. Requiring these structures to be total has been criticised by Lindström and Rabinowicz (1991) and Rott (1992). Lindström and Rabinowicz has considered to drop the total requirement imposed in these structures. One of the most drastic consequences of such relaxation is that the correspondence between these systems is lost. Cantwell (2000) has investigated the issue of using hypertheories, a System of Spheres without the totality requirement, to give semantics to the belief change operators in DDL. He shows that the emerging DDL systems defined in this way are not compact. Unlike us, Cantwell does not consider that the knowledge of an agent is given in a non-compact logic but rather he gives semantics of the belief change operators in non-compact systems. The knowledge of an agent is still constrained to be represented in propositional logics via the doxastic modal operators of DDL.

In this work, we are not interested in represent the knowledge of an agent and define modal operators of belief change in the object language of the logic. We aim to understand the aspect of belief change on knowledge/beliefs expressed in non-compact logics. We expect that our study 
will guide future research in extending DDL and DEL for more general logical systems such as temporal logics, and will have impact on applications for reasoning about action, sensing actions and planning.

\section{Belief Change in Formal Methods}

One of the most relevant logics in AI are temporal logics. These logics are used to represent the knowledge an agent has about the environment, to represent its actions over time and to trace plans in order to achieve its goals. Main areas that use temporal logics are planning and reasoning about actions. Temporal logics are also very important in Formal Methods for formal specification and verification of systems. Some works have considered applying belief change to automatize the process for repairing a system specification when it fails to cope with its requirements. We discuss here these works and their limitations.

In formal specification and verification of systems, one of the most prominent techniques are Model Checking (Clarke et al., 2001) in which a system is modelled via a formal structure, as Kripke structures, used to give semantics to a logic under which the requirements that the system must cope with will be specified. In case of not satisfying its requirements, the model must be repaired.

Zhang and Ding (2008) proposed to apply KM update to repair a single Kripke structure. This approach is called model update. They proposed a set of five primitive change operations to modify a model and define an admissible minimal change criteria. Nonetheless, as pointed by Guerra and Wassermann (2010), model update can lead to significant loss of information in some cases, and it is argued that AGM belief revision presents a suitable apparatus to revise models in static contexts where the requirements do not change, but the model that does not cope with its own specification is revised.

Guerra (2016) has discussed two main aspects involving AGM paradigm regarding temporal logics. First, he showed that the standard constructions for AGM contraction, in particular the partial meet functions, cannot be defined in CTL and LTL, two non-compact logics widely used in model checking. He then proposed how to repair a single Kripke structure in order to maintain some desirable structures and properties of the model. Although Guerra has shown that partial meet functions cannot be defined in such logics, he has not investigated if this implies that no function that satisfies the AGM postulates can be defined on these logics, or only that partial meet functions are too weak in these logics. We address this issue in this thesis, and we show that there are AGM rational contraction functions in these logics.

Both works of Guerra and Zhang and Ding have assumed that the knowledge of an agent is given by a single kripke structure. Relying in this kind of representation is too restrictive, as it imposes an agent to consider a complete knowledge about the environment. Often, however, an agent has only a partial knowledge of the events and circumstances of the environment. For instance, in Example 2, although both Alice and John know their own cards, they are unable to determine which cards his/her opponent is holding.

Gabbay et al. (2008) propose an approach for applying AGM revision in non-classical logics that can be expressed in first-order logics. They consider the modal system $K$, Belnap's four valued logic, and Łukasiewicz's many-valued logic. Their approach consists of a translation from these logics to classical logics, where the revision is performed. 
The rationality postulates of the AGM paradigm have proved to be too strong in some logics. Indeed, it is not even possible to define rational AGM belief change function in logics such as Horn Logic and some classes of Description Logics. To workaround this limitation, Ribeiro (2013) has devised an alternative theory of belief change for logics that do not cope with AGM postulates, precisely classes of Description Logics that are not closed under classical negation.

All these attempts of applying belief change on non-classical logics have considered only compact logics as formalisms for knowledge representation and reasoning.

\subsection{Overview}

We give a brief overview of the subsequent chapters.

In Chapter 2, we review the AGM paradigm, its rationality postulates and its classical constructions, such as partial meet functions, epistemic entrenchment and Grove's System of Spheres. We also review the difficulties involved in applying the AGM paradigm in logics without compactness and why without compactness the classical construction are not AGM rational. We basically review the concept of AGM compliance, introduced by Flouris (2006), which presents necessary and sufficient conditions for the existence of AGM rational contraction functions in a logic. In the subsequent chapter, Chapter 3, we identify a class of non-compact logics that are AGM compliant, and we devise new constructions that are AGM rational for both AGM contraction and AGM revision. We also address the issue of the Levi and Harper identities in the absence of compactness.

In Chapter 4, we review the KM paradigm and the consequences of dispensing with compactness in this System. In this chapter, we take two path in order to cover non-compact logics: we devise class of functions that are fully KM rational, and we identify some issue with the original KM rationality postulates regarding non-finitary languages.

In Chapter 5, we bring to light the connection between AGM revision and non-monotonic reasoning and we devise a new non-monotonic system that reconnects with the AGM revision.

In the final chapter of this thesis, we summarise the main results obtained in this work and their significance. We end by discussing some research directions worth pursuing in future, and some relavant applications,

The proofs in this work have been moved to the appendices. For convenience, we sketch some of the proofs when appropriate in the chapter itself.

In the next section, we introduce the notation employed, as well as some useful definitions and concepts that we will need throughout this thesis.

\subsection{Notation}

Given a set $A$, the power set of $A$ will be denoted as $2^{A}$. We use the terms formula and sentence interchangeably. We will use upper case Roman letters (A, B, X, Y ...) to denote sets, and lower case Greek letter $(\varphi, \psi, \alpha, \beta, \ldots)$ will be used to denote formulae. We will reserve the upper case letter $K$ for a special kind of sets called belief sets, and the Greek lower case letter $\gamma$ to denote a kind of function called selection function. The upper case letter $\Gamma$ is reserved to denote a collection of sets. Propositional symbols will be denoted by lower case Roman letters (p, q, r, ...). The letter 
$M$ will denote a model. The maximal elements of a set $A$ w.r.t a Partial Order $<$ are given by $\max _{<}(A)=\{a \in A \mid \forall b \in A, a \nless b\}$.

We consider a logic as a pair $\langle L, C n\rangle$, where $L$ is a language and $C n: 2^{L} \rightarrow 2^{L}$ is a logical consequence operator that maps a set of formulae to the set of all its inferred formulae. For readability, for any formula $\varphi$, the set $C n(\{\varphi\})$ will often simply be written as $C n(\varphi)$. We will often pretend that the consequence operation $C n$ itself represents a logic when no confusion is imminent. We limit ourselves to logics that are Tarskian, that is, logics whose consequence operator satisfies the following three properties:

1. (Monotonicity): $A \subseteq B$ iff $C n(A) \subseteq C n(B)$;

2. (Idempotence): $C n(A)=C n(C n(A))$;

3. (Inclusion): $A \subseteq C n(A)$;

Apart from being Tarskian, the consequence operation is often granted some other properties in the AGM belief change literature, and they are often dubbed AGM Assumptions.

- (deduction): $\varphi \in C n(A \cup\{\psi\})$ iff $\psi \rightarrow \varphi \in C n(A)$;

- (supraclassicality): if $\varphi$ is a logical consequence of $A$ in classical propositional logic, then $\varphi \in C n(A)$;

- (compactness): if $\varphi \in C n(A)$ then there is a finite subset $A^{\prime}$ of $A$ such that $\varphi \in C n\left(A^{\prime}\right)$.

We will say that a logic $\langle L, C n\rangle$ is closed under classical negation iff the language $L$ is closed under the negation operator, for each formula $\varphi \in L, C n(\varphi) \cap C n(\neg \varphi)=C n(\emptyset)$, and $C n(\{\varphi, \neg \varphi\})=$ $L$. In other words, the negation is interpreted classically. Analogously, the logic is closed under the disjunction if the language is closed under such a connective and it is also classically interpreted, that is, if $\varphi \in C n(X)$ then $\varphi \vee \psi \in C n(X)$, for every $\psi \in L$ and $X \subseteq L$.

One of the inconveniences of dealing with non-compact logics is that it is not possible to define a finite proof system for these logics. We exploit the semantics of the logical consequence operator $C n$ as a remedial measure. Given a logic $\langle L, C n\rangle$ and a set of structures $\mathcal{I}$, an interpretation or a model is an element of $\mathcal{I}$ that gives meaning to the formulae of $L ; \mathcal{I}$ is called an interpretation domain of that logic, whereas each subset of $\mathcal{I}$ is called an interpretation set. A satisfaction relation $\models$ binds each pair in $\mathcal{I} \times L$ to a truth value $T$ (true) or $F$ (false), meaning that a formula is respectively true or false in that model. For instance, an interpretation domain for Propositional Logic is the power set of the propositional symbols of the language.

In Tarskian logics, the consequence operator can be semantically defined as: a formula $\varphi \in$ $C n(X)$ iff every model that satisfies all formulae in $X$ also satisfies $\varphi$. A theory or belief set is a set of formulae logically closed under the consequence operator $C n$, that is, a theory $A$ is a set of formulae such that $A=C n(A)$. We will reserve the letter $K$ to denote theories. Let $\mathcal{I}$ be an interpretation domain of a logic $\langle L, C n\rangle$, and $M$ a model in $\mathcal{I}$. The set of all formulae of $L$ satisfied by $M$ is the theory $T h(M)=\{\varphi \in L \mid M \models \varphi\}$. Generalizing, given a set of models $A, T h(A)=$ $\{\varphi \mid \forall M \in A, M=\varphi\}$ is the theory of the formulae satisfied by all models in $A$. Moreover, given a 
theory $X \subseteq L$, the set of models that satisfy all formulae in $X$ is $\llbracket X \rrbracket=\{M \in I|\forall \varphi \in X, M|=\varphi\}$. For simplicity, given a set of formulae $X$ and a model $M$, we will write $M \models X$ to mean that $M$ satisfies every formulae in $X$. Furthermore, we will call a theory $T$ a complete theory if and only if, for every sentence $\varphi$, either $\varphi \in T$ or $\neg \varphi \in T$, and use $T_{L}$ to denote all such complete theories. Notice that, in logics closed under classical negation, the existence of complete theories is trivial.

A theory is said to be consistent if it does not entail some formula $\varphi$ and its negation $\neg \varphi$. The set of all consistent complete theories that entail a formula $\varphi$ is given by the set $\omega(\varphi)=\left\{T \in T_{L} \mid\right.$ $\varphi \in T\}$. Conversely the set of all consistent complete theories that do not entail a formula $\varphi$ is given by $\bar{\omega}(\varphi)=\left\{T \in T_{L} \mid \varphi \notin T\right\}$. A consistent complete theory from $\bar{\omega}(\varphi)$ will be called a complement of $\varphi$. In logics closed under classical negation the complement of $\varphi$ coincides with the consistent complete theories of its negation, that is, $\bar{\omega}(\varphi)=\omega(\neg \varphi)$. This will prove useful to define the classes of (fully) AGM rational contraction/revision functions.

Observation 1.1. In logics closed under classical negation, $\bar{\omega}(\varphi)=\omega(\neg \varphi)$.

Unlike in Propositional Logic, in non-classical logics, e.g. modal logics, two different models, say $M$ and $M^{\prime}$, may satisfy exactly the same set of formulae, that is, $M \neq M^{\prime}$ but $T h(M)=T h\left(M^{\prime}\right)$. We say that such models are semantically equivalent.

Some of the functions we will define in this work will rely on binary relations over the class of all consistent complete theories. The idea behind these functions is that they will pick, for each formula $\varphi$, the best elements of either $\omega(\varphi)$ or $\bar{\omega}(\varphi)$ according to a binary relation $<\subseteq T_{L} \times T_{L}$. We will call it relational functions. 


\section{Chapter 2}

\section{Belief Systems and the AGM Paradigm}

This thesis is about belief change, but before we proceed to such matter, we shall first discuss about how to represent an agent's body of knowledge and what causes an agent to change its beliefs. This matter is discussed in the section 2.1. Subsequently, in Section 2.2, we review one of the most influential belief change frameworks: the AGM paradigm.

\subsection{Epistemic states, inputs and changes}

Since we want to talk about belief change, we will first need a model capable of talking about beliefs and their dynamics, independent of technicalities. The model should be general enough to allow one to consider many different aspects of knowledge representation and their relation with events that cause an agent (or agents) to change its (their) beliefs.

We can start by imagining that in each instant of time we have a corpus of beliefs (we do not aim to discuss now how these beliefs are represented but rather conceptualise this notion). Let us consider the following illustrative example.

Example 3. At 7:00 AM, Alice has checked the weather forecast which informs that the whole day will be sunny. She decides, then, to commute to her office with her bicycle. At 7:15 AM, however, after finishing her breakfast, Alice looks through the windows and realises that it has just started raining. She immediately changes her mind and decides to take a bus to the office instead.

Alice has two different bodies of knowledge at the example above. First, at 7:00 AM she considers that the whole day will be sunny, and that she will ride a bike to go to the office. Fifteen minutes later, her beliefs has suddenly changed: it is raining, and she will take a bus to the office. The collection of all the beliefs an agent has in an instant of time is called an epistemic state. The notion of epistemic state does not concern how an agent's beliefs are represented, or stored, but rather provide an idealized model of the information it believes in a point of time. The structure used to represent an epistemic state is dubbed an epistemic state model. For instance, in this work we shall constrain ourselves to representing epistemic states as a set of sentences expressed in a suitable language. Other models exists as, for instance, Bayesian models where each piece of information is associate with a probability that measures its level of reliability.

As an agent interacts with the external forces of an environment, it will receive new pieces of information and will be forced to confront them with its own beliefs. For instance, suppose 
that at 7:05 AM Alice receives a phone call from her friend John telling her to take an umbrella before leaving home because he suspects that will rain soon. At that time, Alice simply disregards his advice, since she considers the weather-forecast more reliable than John's self-predictions. In this scenario, Alice's attitude is to reject the new input. The judgement an agent makes about information it confront with its own beliefs is called an epistemic attitude. For instance, suppose a model in which the epistemic states are represented as set of sentences written in a propositional language. There are three different attitudes one may have regarding a sentence $\alpha$ and a epistemic state $A$ : (i) accept $\alpha$, if $\alpha \in A$; reject $\alpha$, in the case that its negation $\neg \alpha$ is in $A$; or be ignorant about the new information, when neither $\alpha$ nor $\neg \alpha$ is in $A$.

The new input an agent receives is called an epistemic input and may cause different effects on the current epistemic state of an agent. For instance, in Example 3 after looking through the windows, Alice realises that it is raining. This input has two main effects on her epistemic state: (i) she adds the new piece of information (raining) and (ii) drops both her beliefs about the weather (from sunny to raining) and the way she will commute to the office (from bike to bus). The new piece of information makes Alice go from one epistemic state to another one. This is called an epistemic action or an epistemic change. According to the adopted model of epistemic state, and epistemic input may have different epistemic attitudes and type of changes.

A system which contains exactly an epistemic state model, a set of epistemic attitudes, a class of epistemic inputs and a group of change operations is called a belief system (Gärdenfors, 1988). In this work, we will focus on the belief systems of the AGM paradigm and KM paradigm. The belief system of the AGM paradigm is reviewed in the following section and the KM belief system is presented in Chapter 4.

\subsection{AGM Paradigm}

The AGM (Alchourrón, Gärdenfors and Makinson) paradigm is the foremost theory of belief change. It was first proposed in (Alchourrón et al., 1985) and further developed in works such as (Gärdenfors, 1988; Hansson, 1999).

In this paradigm, an agent's beliefs are represented via formulae written in the propositional language of an underlying logic $\langle L, C n\rangle$. An epistemic state is given by a belief set (or theory) $K$. We recall from the introductory section that a theory $K$ is a set of formulae logically closed under a consequence relation $C n$, i.e. $K=C n(K)$. The consequence relation is explicitly assumed to be Tarskian, closed under all boolean connectives, compact, and satisfy the supraclassicality and deduction theorem. These properties are often dubbed the AGM assumptions, and were discussed in Chapter 1, Section 1.7.

An agent interacts with its own beliefs and external information via formulae written in the propositional language of the underlying logic. An agent presents three epistemic attitudes when it confronts its own beliefs (an epistemic state $K$ ) against an external piece of information $\varphi$ :

1. accept: if $\varphi \in K$.

2. reject: if $\neg \varphi \in K$.

3. indetermined: if $\varphi$ and $\neg \varphi$ are not present in $K$. 
Let $K$ be the collection of all theories from $L$, then a belief change operation is any function $f: \mathbf{K} \times L \rightarrow \mathbf{K}$ that maps a theory $K$ to a new theory $K^{\prime}$ in the light of a epistemic input $\varphi$. A belief change operation is performed according to the epistemic action an agent might have with respect to an epistemic input $\varphi$ :

1. Expansion $(K * \varphi)$ : adds $\varphi$ to $K$.

2. Contraction $(K-\varphi)$ : removes $\varphi$ from $K$.

3. Revision $(K * \varphi)$ : acquire $\varphi$ keeping the new epistemic state consistent, if $\varphi$ is consistent.

The three kinds of operations are ruled by rationality postulates that prescribe rational behaviours for an agent. Although the expansion operation $K+\varphi$ was originally defined via postulates, as the other two operations (contraction and revision), the expansion consists in taking the closure of the belief set $K$ augmented with the new information $\varphi$, that is, $K+\varphi=C n(K \cup\{\varphi\})$. The contraction and revision operation are presented in the following sections.

\subsubsection{Contraction}

A contraction operation is responsible to successfully relinquish a belief from the current epistemic state of an agent. Removal of beliefs, however, is not easy as it might seem in the first glance. This is because in relinquishing a piece of information $\varphi$, one needs also to expel other beliefs that support $\varphi$. For instance, consider that the current epistemic of an agent is given by the theory $K=C n(\{p, q, q \rightarrow p\})$, and that the agent decides to remove $p$; where $p$ and $q$ are atomic propositional symbols and $C n$ is the classical propositional logic. Simply removing $p$ from $K$ would result in $K^{\prime}=C n(\{q, q \rightarrow p\})$ which does not removes $p$, since $q$ and $q \rightarrow p$ entails $p$ back. To successfully relinquish $p$, more beliefs need to be removed from $K$. Clearly, an agent cannot go rogue and start removing everything from its body of knowledge, which would be too drastic. Some criteria to guide the contraction operation should then be granted. These guidelines are given by rationality postulates that prescribe good behaviours an agent must follow: six basic ones, and two supplementary postulates that concern conjunctive formulae.

$$
\begin{array}{lll}
\mathbf{( \mathbf { K } _ { 1 } ^ { - } )} & K-\varphi=C n(K-\varphi) & \text { (closure) } \\
\mathbf{( \mathbf { K } _ { 2 } ^ { - } )} & K-\varphi \subseteq K & \text { (inclusion) } \\
\mathbf{( \mathbf { K } _ { 3 } ^ { - } )} & \text { If } \varphi \notin K, \text { then } K-\varphi=K & \text { (vacuity) } \\
\left(\mathbf{K}_{4}^{-}\right) & \text {If } \varphi \notin C n(\emptyset), \text { then } \varphi \notin K-\varphi & \text { (success) } \\
\left(\mathbf{K}_{5}^{-}\right) & K \subseteq(K-\varphi)+\varphi & \text { (recovery) } \\
\left(\mathbf{K}_{6}^{-}\right) & \text {If } C n(\varphi)=C n(\psi), \text { then } K-\varphi=K-\psi & \text { (extensionality) }
\end{array}
$$

The postulate $\left(\mathrm{K}_{1}^{-}\right)$has the purpose to guarantee that the contraction outcome is still confined to a theory. The postulates $\left(\mathrm{K}_{2}^{-}\right)$and $\left(\mathrm{K}_{3}^{-}\right)$claim respectively that in a contraction operation no beliefs are added and if the formula being contracted is not in the belief set, then there is nothing to be contracted at all. As the epistemic states are represented by belief sets, the tautologies are present in all epistemic states. Therefore, the tautologies are the only formulae which cannot be 
contracted, otherwise it would violate the closure postulate. In this sense, $\left(\mathrm{K}_{4}^{-}\right)$assures that if a formula is not a tautology, then it will not to be present any more after its contraction, i.e, the contraction must be successfully fulfilled. According to $\left(\mathrm{K}_{5}^{-}\right)$, contracting a sentence from a belief set and expanding it with the same belief should result in the original belief set. Postulate $\left(\mathrm{K}_{6}^{-}\right)$ assures that if two formulae are logically equivalent then both contractions must be the same. The main idea is that the contraction should not be affected by syntactical matters.

There are two supplementary postulates regarding conjunctive formulae:

$$
\begin{array}{ll}
\left(\mathbf{K}_{7}^{-}\right) & K-\varphi \cap K-\psi \subseteq K-\varphi \wedge \psi \\
\left(\mathbf{K}_{8}^{-}\right) & \text {If } \varphi \notin K-(\varphi \wedge \psi), \text { then } K-(\varphi \wedge \psi) \subseteq K-\varphi .
\end{array}
$$

Let us suppose that an agent believes in $\varphi$ and $\psi$, so it also believes in $\varphi \wedge \psi$. To contract $\varphi \wedge \psi$, it suffices to give up just one of them, that is, $\varphi$ or $\psi$. Therefore, all the beliefs in the contraction of $\varphi$ and in $\psi$ are in $K-(\varphi \wedge \psi)$, that is, the beliefs in $K-\varphi$ and in $K-\psi$ must be in $K-(\varphi \wedge \psi)$ as well. This is ruled by postulate $\left(\mathrm{K}_{7}^{-}\right)$. According to $\left(\mathrm{K}_{8}^{-}\right)$, if $\varphi$ is removed when contracting $\varphi \wedge \psi$, then any formula withdrawn in such a contraction also must be absent in $K-\varphi$. It follows from these two last postulates that $K-\varphi=K-(\varphi \wedge \psi)$ or $K-\psi=K-(\varphi \wedge \psi)$ or $K-\varphi \cap K-\psi=K-(\varphi \wedge \psi)$ (Alchourrón et al., 1985).

\section{Contraction Functions}

Although the rationality postulates serve as good guide for belief change, it does not tell us hot to construct belief change functions. A belief change operation that satisfies all six basic AGM postulates of contraction is an AGM rational contraction function. If besides the six postulates, it also satisfies the supplementary ones then we say that is fully AGM rational. We shall visit in this section the two most influential AGM rational contraction functions: Partial Meet and Epistemic Entrenchment.

We begin with the class of the Partial Meet functions. These functions are founded on two principal elements: remainder sets and selection functions.

The remainder set of a belief set $K$ and a sentence $\varphi$ consists of all maximal belief sets in $K$ that do not imply $\varphi$.

Definition 2.1. Let $K$ be a belief set, the class of all remainders of $K$ with respect to a sentence $\varphi$ is the set $K \perp \varphi$ such that a set $X \in K \perp \varphi$ iff

- $X \subseteq K$;

- $\varphi \notin C n(X)$;

- for any subset $Y$ of $K$, if $X \subset Y$ then $\varphi \in C n(Y)$.

The belief sets in $K \perp \varphi$ are taken to contract $\varphi$ from $K$. These sets are chosen according to a selection function which picks up the best sets in a remainder.

Definition 2.2. A selection function $\gamma$ maps a remainder set $K \perp \varphi$ to a subset of it such that 


$$
\gamma(K \perp \varphi)=\left\{\begin{array}{cl}
\emptyset \neq \chi \subseteq K \perp \varphi & \text { iff } K \perp \varphi \neq \emptyset \\
\{K\} & \text { otherwise }
\end{array}\right.
$$

Definition 2.3. Given a belief set $K$, a sentence $\varphi$ and a selection function $\gamma$, a operation - is a partial meet contraction operation iff

$$
K-\varphi=\bigcap \gamma(K \perp \varphi)
$$

Given a selection function $\gamma$, we write $-_{\gamma}$ to denote the resulting partial meet contraction function according to Definition 2.3. Let us take as example the belief set $K=C n(\{p\})$ over the set of propositional symbols $A P=\{p, q\}$. There are two remainders for $K$ w.r.t $p$ :

$$
K_{1}=C n\{p \vee q\} \quad K_{2}=C n(\{p \vee \neg q\})
$$

Thus, we have that $K \perp p=\left\{K_{1}, K_{2}\right\}$. Three possible selection functions to contract $p$ from $K$ are:

$$
\gamma_{1}(K \perp p)=\left\{K_{1}\right\} \quad \gamma_{2}(K \perp p)=\left\{K_{2}\right\} \quad \gamma_{3}=\left\{K_{1}, K_{2}\right\}
$$

Each of the three selection functions produces a different contraction result. Following Definition 2.3, we have that:

$$
\begin{aligned}
& \gamma_{1}: K{ }_{\gamma_{1}} p=\bigcap\left\{K_{1}\right\}=C n(\{p \vee q\}) \\
& \gamma_{2}: K-{ }_{\gamma_{2}} p=\bigcap\left\{K_{2}\right\}=C n(\{p \vee \neg q\}) \\
& \gamma_{3}: K{ }_{\gamma_{3}} p=\bigcap\left\{K_{1}, K_{3}\right\}=C n(\emptyset)
\end{aligned}
$$

Every single partial meet contraction function satisfies all the six basic contraction postulates. Indeed, every function satisfying the six basic postulate is a partial meet contraction function. In other words, the class of partial meet contraction functions is characterized by the six basic postulates for contraction.

Theorem 2.1. (Alchourrón et al., 1985) A function - satisfies $\left(K_{1}^{-}\right)$to $\left(K_{6}^{-}\right)$if and only if - is a partial meet contraction function.

There are two special cases of partial meet contraction functions taken to be too drastic: full meet and maxichoice. In a maxichoice operation, the selection function always picks up only one remainder in $K \perp \varphi$, whereas in the full meet, all remainders are selected. The partial meet contraction function generated from the selection functions $\gamma_{1}$ and $\gamma_{2}$ are examples of maxichoice operators, while the one generated from $\gamma_{3}$ is a full meet contraction function. 
The class of partial meet contraction functions, however, does not guarantee the two supplementary postulates $\left(\mathrm{K}_{7}^{-}\right)$and $\left(\mathrm{K}_{8}^{-}\right)$. To achieve a class of functions characterized by all the eight contraction postulates it is necessary to restrict how a selection function chooses remainders. When contracting, an agent may consider some beliefs more reliable than others, meaning there is preference to keep some sentences over those to be eliminated. Therefore, for every sentence $\alpha$, a selection function should pick up the best remainders in $K \perp \alpha$, in the sense it keeps the most plausible sentences an agent wants to retain. Such a preference may be represented through a relation over the maximal subsets of a theory $K$, in order to make the contraction. A selection function that takes a plausibility order into account is called relational. We address the selection functions which consider transitive preference relations.

Definition 2.4. A selection function $\gamma$ over a theory $K$ is transitively relational if and only if there is a binary transitive relation $\leqslant$ over $2^{K}$ such that

$$
\gamma(K \perp \varphi)=\left\{K^{\prime} \in K \perp \varphi \mid K^{\prime \prime} \leqslant K^{\prime}, \text { for all } K^{\prime \prime} \in K \perp \varphi\right\} .
$$

A partial meet contraction operator whose selection function is transitively relational is called a transitive relational partial meet contraction function. The class of these functions characterizes the eight AGM contraction postulates.

Theorem 2.2. (Alchourrón et al., 1985) A function - is a transitive relational partial meet contraction function iff it satisfies $\left(K_{1}^{-}\right)$to $\left(K_{8}^{-}\right)$.

\section{Epistemic Entrenchment}

Besides partial meet contraction functions, there are other constructive models for AGM contraction, as the Epistemic Entrenchment model. It is natural to think that an agent, in an epistemic state, considers some sentences more reliable than others. Therefore, we may assume that an agent has a relation $\leqslant$ over its beliefs depending on the epistemic state. Let $\varphi$ and $\psi$ be two formulae, $\varphi \leqslant \psi$ means an agent considers $\psi$ at least as entrenched as $\varphi$, and $\varphi<\psi$ denotes that $\psi$ is more reliable (or entrenched) than $\varphi$. Such a relation, clearly, must satisfy some minimal requirements:

$$
\begin{array}{lll}
\text { (EE1) If } \varphi \leqslant \psi \text { and } \psi \leqslant \alpha \text { then } \varphi \leqslant \alpha & \text { (Transitivity); } \\
\text { (EE2) if } \varphi \in C n(\psi) \text { then } \psi \leqslant \varphi & \text { (Dominance); } \\
\text { (EE3) if } \varphi, \psi \in K \text {, then } \varphi \leqslant \varphi \wedge \psi \text { or } \psi \leqslant \varphi \wedge \psi & \text { (Conjunctiveness); } \\
\text { (EE4) if } K \text { is consistent, then } \varphi \notin K \text { iff } \varphi \leqslant \psi \text { for all } \psi \in L & \text { (Minimality); } \\
\text { (EE5) if for all } \psi \in L, \psi \leqslant \varphi \text { then } \varphi \in C n(\emptyset) & \text { (Maximality). }
\end{array}
$$

Condition (EE1) states that the entrenchment relation has to be transitive. According to (EE2), the logically stronger a sentence is, the least entrenched it becomes. For instance, let us consider that an agent believes $(\varphi)$ there is ice-cream in the freezer. Therefore, it also believes that there is ice-cream in the freezer or $(\psi)$ there is no cheese in the freezer. However, when contracting $\varphi$ the agent has no reason to remove $\varphi \vee \psi$, which means the latter is at least as entrenched as the former. It follows from (EE1) and (EE2) that the relation $\leqslant$ is a pre-order (that is, transitive and 
reflexive). According to (EE3), if an agent wants to contract $\varphi \wedge \psi$ then it has to remove $\varphi$ or $\psi$, which means $\varphi \wedge \psi$ is at least plausible as $\varphi$ or $\psi$. The two last postulates deal with the most and least entrenched elements of the the relation: (EE4) informs that all formulae not accepted are the least entrenched; and (EE5) postulates the most entrenched formulae are the tautologies, since they cannot ever be contracted.

A pre-order which satisfies $(E E 1)-(E E 5)$ is called an epistemic entrenchment ordering. We may construct a contraction function from an epistemic entrenchment ordering via condition $(C-)$ :

(C-) $\psi \in K-\varphi$ iff $\psi \in K$ and either $\varphi<\varphi \vee \psi$ or $\varphi \in C n(\emptyset)$.

Indeed, any contraction function constructed from an epistemic entrenchment ordering according to condition $(C-)$ satisfies all eight AGM contraction postulates.

Theorem 2.3. (Alchourrón et al., 1985) Let $\langle K, C n\rangle$ be a logic, and $K$ a belief set of L. A preorder $\leqslant$ over $L$ is an epistemic entrenchment ordering iff the contraction function - constructed according to $(C-)$ satisfies $\left(K_{1}^{-}\right)$to $\left(K_{8}^{-}\right)$.

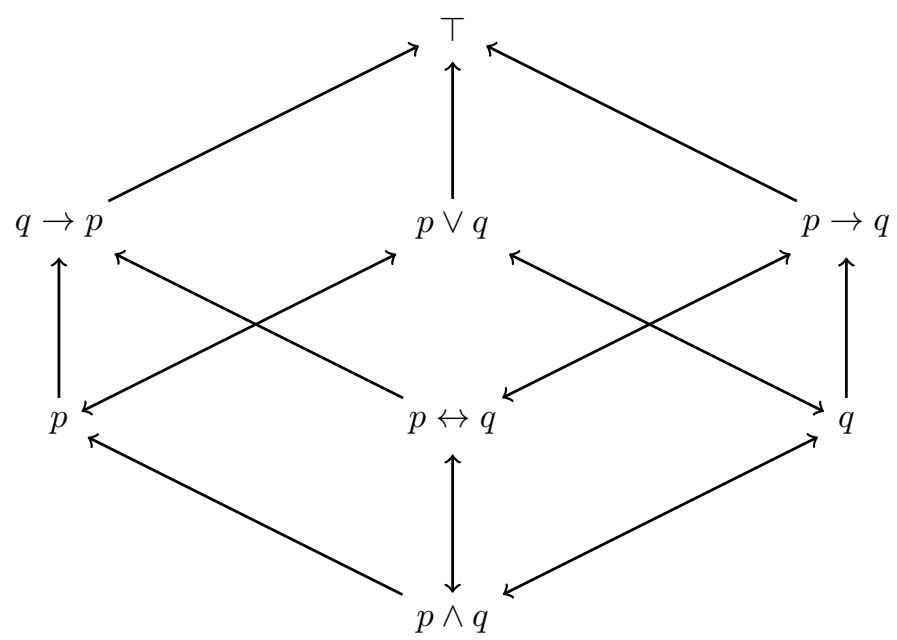

Figure 2.1: An epistemic entrenchment relation.

Example 4. Let $K=\{p, q\}$ be a theory, $\leqslant_{E}$ be the relation illustrated in Figure 2.1 over $K$ such that:

$\left(\leqslant_{E} 1\right)$ for all $\varphi \notin K$ and all $\psi \in L, \varphi \leqslant \psi$.

In Figure 2.1, an arrow $\varphi \rightarrow \psi$ indicates that $\varphi \leqslant_{E} \psi$. Moreover, if $\varphi^{\prime}$ and $\psi^{\prime}$ are respectively logically equivalent to $\varphi$ and $\psi$, then $\varphi^{\prime} \leqslant_{E} \psi^{\prime}$. We can note that $\leqslant_{E}$ satisfies Transitivity (EE1), Dominance(EE2) and Conjunctiveness (EE3), while condition $\left(\leqslant_{E} 1\right)$ ensures (EE4). As the tautological formulae are the maximal ones in $\leqslant_{E}$, it follows from this and (EE4) that $\leqslant_{E}$ satisfies (EE5). Thus, $\leqslant_{E}$ is an epistemic entrenchment relation.

The contraction function $-\leqslant_{E}$ constructed from $\leqslant_{E}$ according to $(C-)$ satisfies $\left(K_{1}^{-}\right)$to $\left(K_{8}^{-}\right)$.

Let us illustrate the contraction of $K$ with $p$ through $-_{\leqslant_{E}}$.

According to $(C-)$, a formula $\varphi \in K-_{\leqslant_{E}} p$ iff $p<_{E}(p \vee \varphi)$ or $p<_{E}(p \vee \varphi)$. The only formulae satisfying this constrain, besides the tautological ones, are those equivalent to $q \rightarrow p, p \rightarrow q$ and $p \leftrightarrow q$. Hence, $K-\leqslant_{E} p=C n(\{q \leftrightarrow p\})$. 


\subsubsection{Revision}

The revision operation consists in adding a new information to a belief set guaranteeing its consistency if the new information is consistent. Six basic postulates rule revision:

$$
\begin{array}{lll}
\left(\mathbf{K}_{1}^{*}\right) & K * \varphi=C n(K * \varphi) & \text { (closure) } \\
\left(\mathbf{K}_{2}^{*}\right) & \varphi \in K * \varphi & \text { (success) } \\
\left(\mathbf{K}_{3}^{*}\right) & K * \varphi \subseteq K+\varphi & \text { (inclusion) } \\
\left(\mathbf{K}_{4}^{*}\right) & \text { If } \neg \varphi \notin K, \text { then } K+\varphi \subseteq K * \varphi & \text { (preservation) } \\
\left(\mathbf{K}_{5}^{*}\right) & K * \varphi=C n(\perp) \text { iff } \neg \varphi \in C n(\emptyset) & \text { (consistency) } \\
\left(\mathbf{K}_{6}^{*}\right) & \text { If } C n(\varphi)=C n(\psi), \text { then } K * \varphi=K * \psi & \text { (extensionality) }
\end{array}
$$

Postulate $\left(\mathrm{K}_{1}^{*}\right)$ informs that the revision must be closed under the logical consequence relation. Postulate $\left(\mathrm{K}_{2}^{*}\right)$ guarantees the new information will be present in the agent's beliefs. Postulates $\left(\mathrm{K}_{3}^{*}\right)$ claims that the agent absorbs no more than the new information and its logical consequences. According to postulate $\left(\mathrm{K}_{4}^{*}\right)$, if the new information is consistent with the previous agent's beliefs then there is nothing to be removed. We highlight that $\left(\mathrm{K}_{4}^{*}\right)$ and $\left(\mathrm{K}_{3}^{*}\right)$, together, imply that if the new information is consistent with the belief set then the revision corresponds to the expansion operation. Postulate $\left(K_{5}^{*}\right)$ has the purpose to keep the belief set consistent. The only exception is when the input information is inconsistent. The postulates $\left(\mathrm{K}_{6}^{*}\right)$ assures that if two formulae are logically equivalent then the revision results must be the same.

There are also two supplementary postulates regarding conjunctive formulae:

$$
\begin{aligned}
& \left(\mathbf{K}_{7}^{*}\right) K *(\varphi \wedge \psi) \subseteq(K * \varphi)+\psi \\
& \left(\mathbf{K}_{8}^{*}\right) \text { If } \neg \psi \notin K * \varphi \text {, then } K * \varphi+\psi \subseteq K *(\varphi \wedge \psi)
\end{aligned}
$$

Revision and contraction can be defined in terms of each other via Levi Identity and Harper Identities. According to Levi identity, revising with a formula $\varphi$ consists in contracting its negation and expanding by $\varphi$. From Harper identity, contracting a belief set is equivalent to intersect the original belief set with the revision by the negation of the input.

(Levi's Identity) $K * \varphi=(K-\neg \varphi)+\varphi$

(Harper's Identity) $K-\varphi=K \cap(K * \neg \varphi)$

This means that, contraction operations as partial meet and epistemic entrenchment may be used to construct revision operators. Any revision function constructed from a partial meet function via Levi Identity is called partial meet revision function. Analogously, we call epistemic entrenchment revision functions those constructed from epistemic entrenchment contraction functions, though Levi Identity.

Theorem 2.4. (Gärdenfors, 1988) If a contraction function - satisfies $\left(K_{1}^{-}\right)$to $\left(K_{4}^{-}\right)$and $\left(K_{6}^{-}\right)$, then the revision function produced through Levi Identity satisfies $\left(K_{1}^{*}\right)$ to $\left(K_{6}^{*}\right)$. 
Theorem 2.5. (Alchourrón et al., 1985) Let - be a contraction function - satisfying $\left(K_{1}^{-}\right)$to $\left(K_{4}^{-}\right)$and $\left(K_{6}^{-}\right)$, and $*$ a revision function constructed via Levi Identity. If - satisfies $\left(K_{7}^{-}\right)$, then $*$ satisfies $\left(K_{7}^{*}\right)$; and if - satisfies $\left(K_{8}^{-}\right)$then $*$ satisfies $\left(K_{8}^{*}\right)$.

Theorem 2.6. (Gärdenfors, 1988) If a revision function satisfies $\left(K_{1}^{*}\right)$ to $\left(K_{6}^{*}\right)$, then the contraction function - constructed from - through Harper Identity satisfies $\left(K_{1}^{-}\right)$to $\left(K_{6}^{-}\right)$.

Theorem 2.7. (Gärdenfors, 1988) Let $*$ be a revision function satisfying $\left(K_{1}^{*}\right)$ to $\left(K_{6}^{*}\right)$, and contraction function constructed from * through Harper Identity. If * $\left(K_{7}^{*}\right)$, then - satisfies $\left(K_{6}^{-}\right)$, and if $*$ satisfies $\left(K_{8}^{*}\right)$ then - satisfies $\left(K_{8}^{-}\right)$.

Example 5. Let $*$ be the revision function constructed through Levi Identity from the contraction epistemic entrenchment function $-_{\leqslant_{E}}$ in Example 4, for the theory $K=C n(\{p, q\})$. We have

$$
\begin{aligned}
K * \neg p & =\left(K-\leqslant_{E} \neg \neg p\right)+\neg p \\
& =\left(K-\leqslant_{E} p\right)+\neg p \\
& =C n(\{q \leftrightarrow p\})+\neg p \\
& =C n(\{\neg p, \neg q\}) .
\end{aligned}
$$

\section{Grove's System of Spheres}

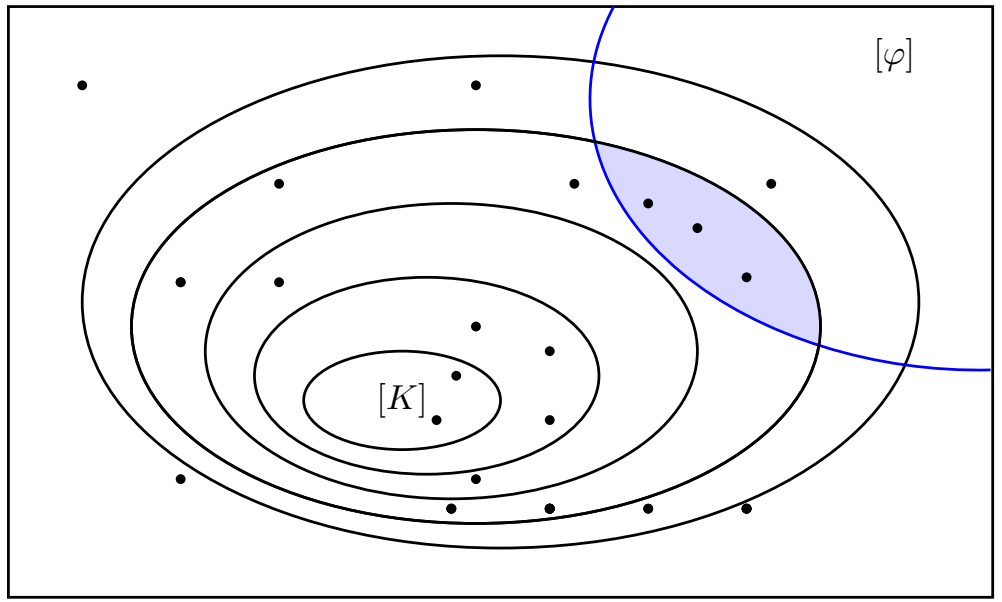

Figure 2.2: A System of Spheres.

The System of Spheres is a constructive model for AGM revision functions proposed by Grove in (Grove, 1988). As in epistemic entrenchment, a system of spheres consists of a preorder. However, the ordered elements are sets of maximal theories, instead of sentences. The main ideia is that there is a plausibility ordering over the theories in an agent's belief set, and such an ordering may be used to revise the belief set with the new information. Before introducing the system of spheres, we present and review some basic definitions and notations which will be used to present the model.

Definition 2.5. Let $\langle L, C n\rangle$ be a logic such that $L$ has the classical negation. A theory $K$ of $L$ is complete iff for all sentence $\varphi \in L, \varphi$ or $\neg \varphi$ is in $K$. Moreover, $K$ is a complete consistent theory if and only if its is complete and for any sentence $\varphi \in L, \varphi$ or $\neg \varphi$ is not in $K$. 
We denote by $\mathbb{M}_{L}$ the set of all consistent complete theories of a logic $\langle L, C n\rangle$. A sphere is any set of complete theories in $\mathbb{M}_{L}$, and the sphere of a theory $K$ is given by the set

$$
[K]=\left\{M \in \mathbb{M}_{L} \mid K \subseteq M\right\}
$$

In order to simplify, given a sentence $\varphi$ we simply write $[\varphi]$ to represent the sphere of the theory $C n(\{\varphi\})$ instead of $[C n(\{\varphi\})]$.

Definition 2.6. Let $K$ be a theory, a system of spheres centred on $[K]$ is a collection $S \subseteq \mathbb{M}_{L}$ such that:

(S1) $S$ is totally ordered w.r.t $\subseteq$;

(S2) $[K]$ is the smallest sphere in $S$, that is, $[K] \in S$ and if $V \in S$ then $[K] \subseteq V$;

(S3) $\mathbb{M}_{L}$ is the largest element in $S$;

(S4) for every sentence $\varphi \in L$, if there is a sphere in $S$ intersecting $[\varphi]$ then there is a smallest sphere in $S$ intersecting $[\varphi]$.

Postulate (S1) restricts the order to be total, which means any two spheres are comparable in terms of plausibility. According to (S2), all spheres in the system are at least as plausible as $[K]$. From (S3), all possible theories must also be considered in the system. Finally, postulate (S4) guarantees that there is always a smallest sphere in the intersection between a system of spheres and the sphere of a consistent formula. It also informs that in a system of spheres there is not an infinity decreasing chain between two spheres.

A system of spheres can be illustrated as in Figure 2.2. Each ellipse represents a sphere, and the points are consistent complete theories. The painted area represents the theories in the intersection of the smallest sphere intersecting $[\varphi]$.

From $S 4$ there is always a smallest sphere in any sphere system which intersects $[\varphi]$, so let $C_{K}(\varphi)$ denote the smallest sphere in a sphere system centred in $[K]$ intersecting $[\varphi]$. To construct a revision function we may consider the most plausible spheres in a sphere system. Thus, we can construct a revision function $*$ as defined in $(S *)$ :

$$
K * \varphi=\left\{\begin{array}{cl}
\bigcap\left(C_{K}(\varphi) \cap[\varphi]\right) & \text { if } \varphi \text { is consistent } \\
L & \text { otherwise }
\end{array}\right.
$$

Any sphere system together with $(S *)$ produces only revision functions that obey $\left(\mathrm{K}_{1}^{*}\right)$ to $\left(\mathrm{K}_{8}^{*}\right)$. Theorem 2.8 ((Grove, 1988)). Let $K$ be a theory, and $S$ a system of spheres centred on $[K]$. A revision function $*$ satisfies $\left(K_{1}^{*}\right)$ to $\left(K_{8}^{*}\right)$ iff there is a system of spheres $S$ centred on $[K]$ such that * respects $S *$ according to the system $S$.

Figure 2.3 illustrates a system of spheres centred on $K=C n(\{p, q\})$ intersecting the sphere $[\neg p]$. According to $S *$, the resulting revision function for $K * \neg p$ is $C n(\{\neg p, \neg q\})$ which coincides with the revision function of Example 5. 


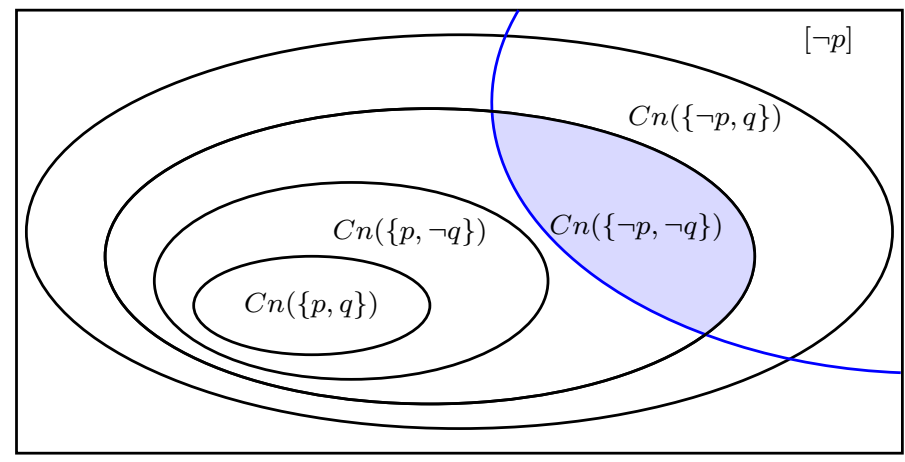

Figure 2.3: A system of Spheres for the function of Example 5.

\subsection{AGM Compliance}

The AGM theory dictates what revisions and contraction functions must satisfy and was originally proposed for logics which satisfy the AGM assumptions - we discussed the AGM assumptions in Section 2.2. However, as shown in (Ribeiro, 2013), in some interesting logics, such as Description Logics (Baader et al., 2003), Horn Logic (Horn, 1951) and Intuitionistic Logic (Heyting, 1956), there is no contraction and revision function satisfying all the AGM postulates. Indeed, these logics are not closed under classical negation (one of the AGM assumptions). In (Ribeiro, 2013) an adaptation of the AGM theory is proposed for these three non-classical logics, dropping some AGM postulates and inserting new ones.

One question which may arise is: does the existence of contraction and revision functions in a logic really require the AGM assumptions? This question was answered by Flouris (2006), in which it is shown that actually the presence of AGM contraction functions is intimately related with the logical property called decomposability, which is introduced in the same work. Flouris called logics for which there exists an operation satisfying the AGM postulates for contraction as AGM-compliant.

To the best of our knowledge, all logics so far proved to be AGM compliant are compact. In this work we are interested in applying AGM paradigm to logics without compactness. Therefore, we have to verify if it is possible to construct revision AGM rational functions.

\subsubsection{Decomposability and AGM Compliance}

We say that a logic complies with the AGM theory if there is at least one contraction function satisfying $\left(\mathrm{K}_{1}^{-}\right)$to $\left(\mathrm{K}_{6}^{-}\right)$. As a consequence, there is also a revision function satisfying $\left(\mathrm{K}_{1}^{*}\right)$ to $\left(\mathrm{K}_{6}^{*}\right)$, via Levi Identity (see Section 2.2.2), if there is negation in such a logic.

Definition 2.7. A logic is AGM-compliant iff there is a contraction operation in such a logic that satisfies $\left(K_{1}^{-}\right)$to $\left(K_{6}^{-}\right)$. Moreover, a logic is fully AGM-compliant iff there is a contraction operation that satisfies $\left(K_{1}^{-}\right)$to $\left(K_{8}^{-}\right)$.

If a logic is AGM-compliant or fully AGM-compliant, than we expect to construct AGM revision functions through Levi's identity. As shown by Flouris (2006), AGM-compliance concerns the existence of relative complementary sets in the logic. 
Definition 2.8. Let $\langle L, C n\rangle$, and $A, K \in 2^{L}$ such that $K=C n(K)$ and $C n(\emptyset) \subset A \subset K$. The complement of $A$ relative to $K, K^{-}(A)$, is the collection of all sets $K^{\prime}$ such that

- $C n\left(K^{\prime}\right) \subset C n(K)$;

- $C n\left(K^{\prime} \cup A\right)=C n(K)$.

To illustrate the intuition of the relative complement concept, let us take the theory $K=$ $C n(\{p, q\})$ over the set of atomic symbols $\{p, q\}$, and $A=C n(\{p\})$. Clearly, $C n(\emptyset) \subset A \subset K$. If we take the set $K^{\prime}=C n(\{p \rightarrow q\}) \subset C n(K)$, then $C n\left(K^{\prime} \cup A\right)=K$. Thus, $K^{\prime} \in K^{-}(A)$, that is, it is in a complement of $A$ relative to $K$. Other sets as $C n(\{q\})$ and $C n(\{p \leftrightarrow q\})$ are also in $K^{-}(A)$.

Definition 2.9. A logic $\langle L, C n\rangle$ is decomposable iff for every $A, K \in 2^{L}$ such that $K=C n(K)$ and $C n(\emptyset) \subset A \subset K, K^{-}(A) \neq \emptyset$.

As stated in Theorem 2.9, decomposability has a straightforward relation with AGM-compliance.

Theorem 2.9. (Flouris, 2006) A logic $\langle L, C n\rangle$ is decomposable iff it is AGM-compliant

In (Ribeiro et al., 2013), the authors studied the connection between some logical properties and AGM-compliance. They also showed that some non-classical logics do not satisfy the AGM theory due to the controversial recovery postulate which is criticised in (Fuhrmann, 1991) and (Hansson, 1991). To notice why, let us take the Example 6 from (Hansson, 1999).

Example 6. My friend John believes that Cleopatra had a son $(\varphi)$ and that Cleopatra had a daughter $(\psi)$. Thus, John also believes that Cleopatra had a child $(\alpha=\varphi \vee \psi)$. Let $K$ be John's belief set. Then he receives an information that makes him relinquish $\alpha$, that is, $K-\alpha$. Some hours later, Johns receives from a reliable source that Cleopatra indeed has a child. Then it is perfectly reasonable for John to add $\varphi \vee \psi$ without reintroducing $\psi$ or $\varphi$ again to his beliefs which contradicts the recovery postulate.

As the recovery postulate presents some problems, it seems reasonable to change it for an weaker postulate, such as the relevance postulate.

$(R-)$ if $\varphi \in K \backslash(K-\psi)$ then there is a $K^{\prime}$ such that $K-\psi \subseteq K^{\prime} \subseteq K, \psi \nsubseteq C n\left(K^{\prime}\right)$ and $\psi \subseteq C n\left(K^{\prime} \cup\{\varphi\}\right)$ (Relevance).

The intuition behind relevance is that in contracting a theory $K$ by a sentence $\psi$, a formula $\varphi$ cannot be removed unless it contributes to entail $\psi$. When changing recovery by relevance, other contractions functions emerge and it is useful to identify in which logics these functions can be defined. We call these logics relevance-compliant.

Definition 2.10. A logic is relevance-compliant if and only if for every theory $K$ in such logic there is a contraction operator that satisfies $\left(K_{1}^{-}\right)$to $\left(K_{4}^{-}\right),\left(K_{6}^{-}\right)$and $(R-)$.

Logics such as Horn Logic and Intuitionistic Logic are relevance-compliant, though they are not AGM-compliant. Indeed, it only requires a logic to be compact in order to be relevance-compliant.

Theorem 2.10. (Ribeiro et al., 2013) If a logic is compact, then it is also relevance-compliant. 


\begin{tabular}{lccc} 
Logic & AGM-compliant & Compact & Relevance-compliant \\
\hline Horn & no & yes & yes \\
Intuitionistic logic & no & yes & yes \\
ALC-ALCIQ & yes & yes & yes \\
SROIQ & no & yes & yes \\
\hline
\end{tabular}

Table 2.1: (Ribeiro et al., 2013) Some logics and their relation with AGM and relevance-compliance.

Table 2.1 presents some logics which are AGM-compliant and some which are not. The logics $S R O I Q$ and $A L C-A L C I Q$ are examples of Description Logics. The proofs that the logics in Table 2.9 are AGM-compliant can be found in (Ribeiro et al., 2013). We highlight that all these logics are compact, unlike CTL. As a consequence, all of them are relevance-compliant. Moreover, alternative belief revision theories devised so far take into account only compact logics (Ribeiro, 2013; Ribeiro et al., 2013). 


\section{Chapter 3}

\section{Dispensing with Compactness in the AGM Paradigm}

Firsts attempts considering belief change in the context of non-compact logics were made by (Guerra, 2016) and (Ribeiro et al., 2013). Both works have considered the connection between Partial Meet functions and the AGM rationality postulates in logics that are not compact. Whereas Ribeiro et al. (2013) show that the representation theorem between Partial Meet functions and the basic AGM contraction postulates cease, Guerra (2016) shows something even worse: Partial meet functions cannot be defined in non-compact logics such as CTL and LTL. These impossibility results, however, bring more questions than answers. They tell us that extending AGM paradigm for logics without compactness is harder than it seems, but they do not give us any hint of the causes for these impossibility results. Precisely, it does not answer a fundamental question: why the connection between the standard constructions for AGM contraction and AGM rationality postulates cease to exist in the absence of compactness? There are two main possible answers for this:

(i) either compactness is so strong that it is not possible to define AGM rational contraction functions without compactness, or

(ii) maybe compactness works as a cornerstone that sustains Partial Meet functions and other equivalent constructions, such as Grove's System of Spheres and Epistemic Entrenchment, to connect with the AGM rationality postulates.

In this chapter, we shall answer such a question. Precisely, we will show that AGM rational contraction functions can still be defined independently of compactness, as long as some few conditions are satisfied. We then provide a complete account of AGM belief change for logics without compactness. In Section 3.1, we identify classes of AGM compliant logics independently of compactness. In the subsequent sections, Section 3.2 and 3.4, we show (fully) AGM rational functions for both contraction and revision. In the last section, we revisit Levi and Harper Identities, and we show that the inter-determinability of contraction and revision functions do not depend on compactness. The full proofs of this chapter were moved to the appendices. Due to the deep length of these proofs, we decided to split them in three chapters of the appendices: Appendix A contains the proofs of Sections 3.1 to 3.3 related to AGM contraction; Appendix B comprises the proofs of Section 3.4, and finally Appendix $\mathrm{C}$ has the proofs of Section 3.5. The results of this Chapter partially appear in (Ribeiro et al., 2018). 


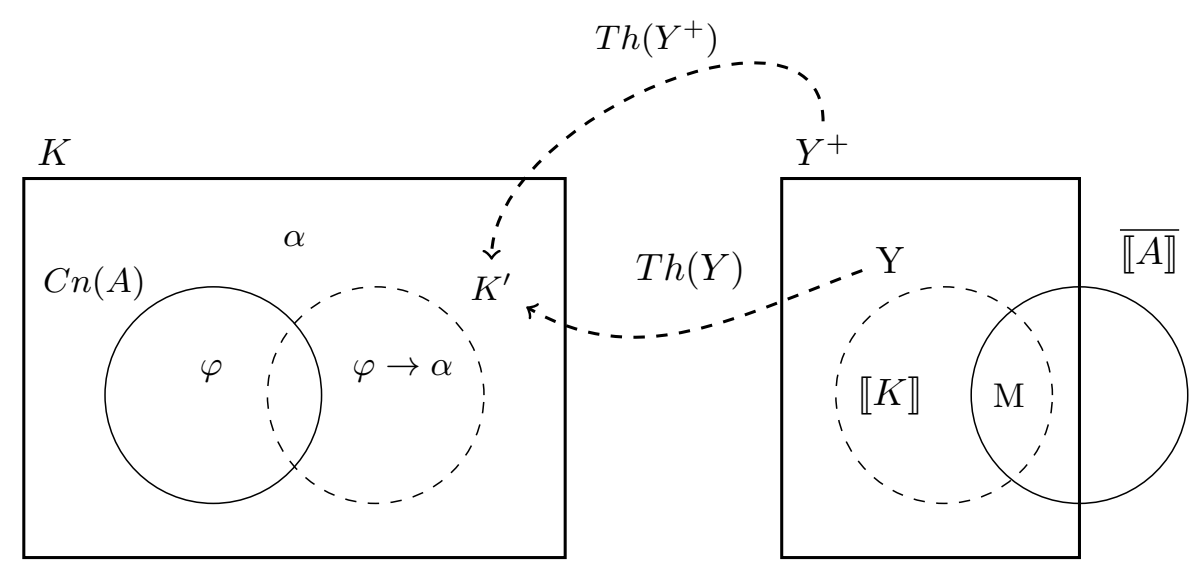

Figure 3.1: A schemata to construct a relative complement $K^{\prime}$ for a theory $K$ and $A$.

\subsection{AGM compliance without compactness}

The purpose of this section is to show that AGM compliance does not depend on compactness. We will only require a logic to be Tarskain and closed under classical negation and disjunction. We recall the functions $\llbracket \cdot \rrbracket$ and $T h(\dot{)}$ introduced in Section 1.7 .

To show that a logic is AGM compliant, we will need to show that it is decomposable. In a broad way, this requires us to show that given a theory $K$ and proper subset $A$ of it. There is a theory $K^{\prime}$ such that the theory that emerges by assembling $A$ and $K^{\prime}$ together matches with $K$, that is, $K=C n\left(A \cup K^{\prime}\right)$. The set $K^{\prime}$, as discussed in Chapter 2 is called a relative complement. The big issue here is to find a way to construct a relative complement $K^{\prime}$. There are another technical details, such as requiring that $A$ is not formed solely by tautologies, and $K^{\prime}$ being a subset of $K$. For now, these technicalities are not important and we will focus only in a general way to achieve a relative complement. We will return later for these technicalities and deal with them properly.

Our main idea, to construct a relative complement, is to resort to the realm of the interpretations of the logic. The idea is as follow, and we use the schemata depicted in Figure 3.1 to support the intuition. We take the models of a theory $K$ and we insert a model $M$ that does not satisfies $A$ (a model from $\overline{\llbracket A \rrbracket}$, for short). Let such a set be $Y=\llbracket K \rrbracket \cup\{M\}$. We argue that the theory $T h(Y)$ is a relative complement of $K$ w.r.t $A$. Showing that $T h(Y)$ is a relative complement is a little bit tricky and we will need some tools of immediate assistance for this. The first one is exhibited in the following observation.

Observation 3.1. Let $K$ be a theory, and $A$ a proper subset of $K$ such that $C n(A) \subset C n(K)$. Moreover, let $Y=[K] \cup\{M\}$, where $M$ is an interpretation. If $M \not \models \varphi$, then for every formula $\alpha \in K$, there is some $\varphi \in A$ such that $\varphi \rightarrow \alpha \in T h(Y)$.

Proof Sketch. Let $\alpha$ be a formula from $K$, we will show that $\varphi \rightarrow \alpha \in T h(Y)$, for some formula $\varphi \in A$. As $Y=\llbracket K \rrbracket \cup\{M\}$, where $M \not \models \varphi$, we get that for some formula $\varphi \in A, M \not \models \varphi$. Let $M^{\prime} \in Y$, we have then that either (i) $M^{\prime} \in \llbracket K \rrbracket$ or $M^{\prime}=M$. In either case, $M^{\prime} \models \varphi \rightarrow \alpha$. Therefore, $\varphi \rightarrow \alpha \in T h(Y)$.

The observation above states that for each formula $\alpha$ of $K$, the theory of $Y$ contains an arrow $\varphi \rightarrow \alpha$, where $\varphi$ is a formula from $A$. In the observation above $Y$ stands for the set of interpretations 
which we are using to construct the relative complement of $K$ w.r.t $A$, as depicted in Figure 3.1. Broadly speaking, this observation tell us that assembling $T h(Y)$ and $A$ gives us $K$ back. Observation 3.1 alone, however, does not guarantee that we do not get more formulae than those in $K$. For this, we will need to explore one more property: the reverse-monotonicity

Reverse-monotonicity: given a function $f$, if $X \subseteq Y$ then $f(Y) \subseteq f(X)$.

Reverse-monotonicity is the opposite of monotonicity. Given a function $f$, it basically states that the bigger a set $A$ is, the smaller will be the outcome of $f(A)$. A good example of a reversemonotonic function is the Th function. This is because, as we can see in the following proposition, the more models a set $A$ has, the smaller is its theory $T h(A)$.

Proposition 3.1. Let $X$ and $Y$ be two sets of interpretations. If $X \subseteq Z$ then $T h(Z) \subseteq T h(X)$.

The following useful observation is straightforward. It basically states that if one departs from a theory $K$ to the set of all its interpretation $\llbracket K \rrbracket$, the theory yielded by such a set of interpretations corresponds exactly to $K$ itself. For instance, let $Y^{+}$in Figure 3.1 stand for $\llbracket K^{\prime} \rrbracket$, thus we would get $K^{\prime}=T h\left(\llbracket Y^{+} \rrbracket\right)$.

Observation 3.2. Given a theory $K, T h(\llbracket K \rrbracket)=K$.

Now we have all the ingredients to show the main result of this section:

Theorem 3.1. Every Tarskian logic closed under classical negation and disjunction is AGMCompliant.

Proof Sketch. Let $K$ be a theory and $A$ a proper subset of $K$ such that $C n(A) \neq C n(\emptyset)$. We will show that there is a relative complement $K^{\prime}$ of $K$ w.r.t A. Precisely, we will show that (i) $C n\left(K^{\prime}\right) \subset K$ and (ii) $C n\left(A \cup K^{\prime}\right)=K$.

As $A \neq C n(\emptyset)$, we get that $A$ has at least one non-tautological formula $\psi$. Thus, there is at least a model $M$ that does not satisfies $\psi$ and therefore $M$ does not satisfies $A$. We proceed with our schemata explained in this chapter (respectively illustrated in Figure 3.1). Let us make

$$
Y=\llbracket K \rrbracket \cup\{M\},
$$

and let $K^{\prime}=T h(Y)$. We proceed to show that we have the items (i) and (ii):

(i) $C n\left(K^{\prime}\right) \subset K$. As $Y \subseteq \llbracket K \rrbracket$, we get from Proposition 3.1 and Observation 3.2 that $T h(Y) \subseteq$ $K$. Since $M$ does not satisfy $A$, we have that for at least one formula $\varphi \in A, M \not \models \varphi$ which implies that $\varphi \notin T h(Y)$. Thus, $T h(Y) \subset K$, and as $T h(Y)=K^{\prime}$, we get $C n\left(K^{\prime}\right) \subset K$.

(ii) $C n\left(A \cup K^{\prime}\right)=K$. We will show (a) $K \subseteq C n\left(A \cup K^{\prime}\right)$ and (b) $C n\left(A \cup K^{\prime}\right) \subseteq K$. The case (b) is straightforward from the monotonicity of $C n$. For the case (a), let $\alpha$ be a formula from $K$, we need to show that $\alpha \in C n\left(K^{\prime} \cup A\right)$. From Observation 3.1 we get that there is a formula $\varphi \in A$, such that $\varphi \rightarrow \alpha \in T h(Y)=K^{\prime}$. Thus, both $\varphi$ and $\varphi \rightarrow \alpha$ are in $\operatorname{Cn}\left(K^{\prime} \cup A\right)$ which implies that $\alpha \in C n\left(K^{\prime} \cup A\right)$. 
From (i) and (ii) we have that $T h(Y)=K^{\prime}$ is a relative complement. Therefore, logics closed under classical negation and disjunction are AGM compliant.

On the next sections, we will devise new classes of contraction and revision functions that are AGM rational. In order to devise these classes we will need some strong tools which we introduce in the rest of this section. The most important one is to find a way to navigate freely between theories and sets of interpretation. For instance, from Proposition 3.1, we know that given two set of interpretations $X$ and $Y$, the theory of $X$ is bigger than the theory of $Y$, as long as $X \subseteq Y$. There is no guarantee, however, that the converse is true. Precisely, we cannot affirm that from $T h(Y) \subseteq T h(X)$ we have $X \subseteq Y$. To see why, suppose two different models $M$ and $M^{\prime}$ such that $T h(M)=T h\left(M^{\prime}\right)$. In this case, though $T h(M) \subseteq T h\left(M^{\prime}\right)$, we do not have $\left\{M^{\prime}\right\} \subseteq\{M\}$. This is precisely what happens in modal logics: two different models can satisfy exactly the same set of formulae.

To be able to navigate back and forth from theories and interpretation sets, we will need to restrict ourselves to a specific set of interpretations. What we need is the density property below.

Definition 3.1. Let $D$ be a domain, and $C_{D}$ be a contra-domain such that $C_{D}$ is a collection of sets. Moreover, let $\Theta: 2^{D} \rightarrow C_{D}$ be a map from the sets within $D$ to the sets of $C_{D}$. A set $A$ in $D$ is $\Theta$-dense iff $\Theta(A \cup\{S\}) \subset \Theta(A)$, for every $S \notin A$.

In a simple way, a set $A$ is dense if it is overfilled with all collections which assembled conserve a desired property $\Theta$. To make it clearer, let us consider for instance the operation $T h$ and a collection $A$ of interpretation sets. Additionally, let us assume that $A$ contains all and only the models that satisfy a formula $\varphi$. Therefore, $\operatorname{Th}(A)=C n(\varphi)$. This means that a model $S$ that is not in $A$ will not satisfy $\varphi$. Therefore, $T h(A \cup\{S\}) \subset T h(A)$. This means that $A$ is $T h$-dense. In a simpler way, $A$ is overfilled with all those models that entail $\varphi$, which means that addition of any model not in $A$ will remove some formula from $T h(A)$. We say that $A$ is dense w.r.t the $T h$ operation within the realm of interpretations. An interpretation set that is $T h$-dense will be called a Complete Interpretation Set (CIS). One can see a CIS as the biggest set of models that satisfies a set of formulae. Notice that, for each set of formulae $X$, the set of all its models, which is given by $\llbracket X \rrbracket$, is a CIS. From this,

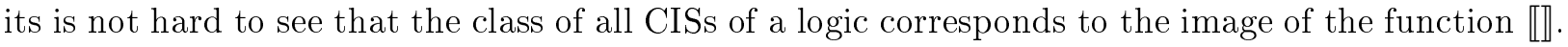

Observation 3.3. Every $\llbracket X \rrbracket$ is a CIS, where $X$ is a set of formulae.

Proposition 3.2. An interpretation set $A$ is a CIS iff $A=\llbracket X \rrbracket$, for some set of formulae $X$.

Density is not constrained for only interpretation sets and the $T h$ operation. In this thesis, we will explore density over other operations and domains, as for instance the intersection operation on the realm of complete consistent theories.

An interesting aspect about density is that it conserves reverse-monotonicity strictly.

Corollary 3.1. Given two $\Theta$-dense sets $A$ and $B$. If $\Theta$ is reverse-monotonic and $A \subset B$, then $\Theta(B) \subset \Theta(A)$.

An immediate property that we gain from density is that we can define a function that maps each theory $K$ to exactly a single CIS, and the theory that follow from such a CIS coincides exactly 
with $K$. In other words, we are able to define a bijection between the realm of theories and CISs. This bijection will be useful to define AGM rational functions on the next sections.

Theorem 3.2. Let $\mathcal{T}$ be the the set of all theories from a logic $\langle L, C n\rangle$, and $\mathcal{I}_{C}$ the class of all complete interpretation sets of that logic. Then the function $\tau: \mathcal{T} \rightarrow \mathcal{I}_{C}$ as $\tau(K)=\llbracket K \rrbracket$ is a bijection.

The bijection we just defined will provide us with some properties that will be useful to construct AGM rational functions for non-compact logics in the next sections. These properties are shown in the observations, corollaries, and propositions below, and their proofs are quite straightforward.

We know, so far, that CISs are $T h$-dense. In the converse direction, the theories are dense w.r.t the function $\llbracket \rrbracket$ which in turn is reverse-monotonic.

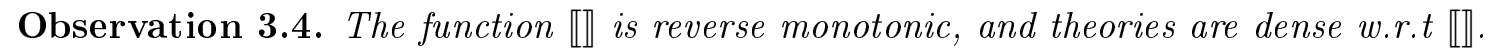

A straightforward consequence from the observation above is that the function $\tau$ that we defined on Theorem 3.2 is reverse-monotonic:

Corollary 3.2. The function $\tau$ from Theorem 3.2 is reverse-monotonic.

The next property is a directly consequence of the observation and corollary above and regards the connection of reverse-monotonicity between theories and CISs. Putting in a simple way, the following proposition claims that the bigger a theory is the smaller is its CISs, and vice versa.

Proposition 3.3. Given two theories $K$ and $K^{\prime}, K \subseteq K^{\prime}$ iff $\llbracket K^{\prime} \rrbracket \subseteq \llbracket K \rrbracket$.

An important consequence of the reverse-monotonicity between CISs and theories (given by the bijection $\tau$ ) is that since a CIS is a dense set, addition of models will immediately imply in the loss of formulae in the corresponding theory. Analogously, going from a theory to a bigger one will imply in loss of models.

Proposition 3.4. Let $A$ and $B$ be two $C I S s$, and $K$ and $K^{\prime}$ two theories:

(1) $K \subset K^{\prime}$ iff $\llbracket K^{\prime} \rrbracket \subset \llbracket K \rrbracket ;$

(2) $\operatorname{Th}(A) \subset T h(B)$ iff $B \subset A$.

Notice that the condition (2) on the proposition above is equivalent to (1), it only makes use of the bijection between CISs and theories to make it explicitly that the strictly bigger is a CIS the strictly smaller is its theory.

The reverse-monotonicity between theories and models yield some interesting behaviours interconnecting the intersection and union operations between these two realms. The first three properties we explore are presented on the following proposition.

Observation 3.5. Given two theories $K$ and $K^{\prime}$, and a model $M$ :

1. $M \models K \cup K^{\prime}$ iff $M \in \llbracket K \rrbracket \cap \llbracket K^{\prime} \rrbracket ;$

2. $\llbracket K \cup K^{\prime} \rrbracket=\llbracket K \rrbracket \cap \llbracket K^{\prime} \rrbracket$; 
3. $\llbracket C n\left(K \cup K^{\prime}\right) \rrbracket=\llbracket K \rrbracket \cap \llbracket K^{\prime} \rrbracket$.

A straightforward consequence from the condition (3) of the observation above and the bijection between CISs and theories is presented in the following proposition. It basically states that the closure taken from assembling two theories $K$ and $K^{\prime}$ corresponds to the theory obtained by intersecting the CISs of $K$ and $K^{\prime}$. Conversely, the intersection of two theories corresponds to theory of assembling the CISs of such two theories.

Proposition 3.5. Given two theories $K$ and $K^{\prime}$,

1. $T h\left(\llbracket K \rrbracket \cap \llbracket K^{\prime} \rrbracket\right)=C n\left(K \cup K^{\prime}\right)$;

2. $T h\left(\llbracket K \rrbracket \cup \llbracket K^{\prime} \rrbracket\right)=K \cap K^{\prime}$.

An immediate consequence of Proposition 3.5 is that the intersection of a set of theories corresponds to the union of the CIS of the theories being intersected:

Corollary 3.3. $\llbracket \cap X \rrbracket=\bigcup_{Y \in X} \llbracket Y \rrbracket$, for all sets of theories $X$.

The following corollary is a trivial property from Tarskian logics, but it will be worth highlighting here as it will come in handy throughout this chapter.

Corollary 3.4. Let $A$ and $B$ be two set of formulae, then $C n(A \cup B)=C n(A \cup C n(B))$.

\subsection{Construction: AGM Contraction Basic Rationality}

In the previous section we showed that Tarskian Logics closed under classical negation and disjunction are AGM-Compliant. A question arises: which class of contraction functions is characterised by the AGM postulates in such logics? Unfortunately, we can no longer rely on partial meet contraction functions for this purpose since, as pointed out in (Ribeiro et al., 2013) partial meet functions do not satisfy the AGM postulates in some non-compact logics. We need to devise a new class of functions.

In this section, we construct a new contraction function which satisfies the postulates $\left(\mathrm{K}_{1}^{-}\right)$to $\left(\mathrm{K}_{6}^{-}\right)$. Recall that $T_{L}$ denotes the set of all consistent complete theories of a logic $\langle L, C n\rangle$, an agent's belief set $K$ is closed under the consequence operation $C n$, and $\bar{\omega}(\varphi)$ is the set of all consistent completes theories that do not entail $\varphi$ (the complements of $\varphi$ ). In the AGM approach the partial meet contraction depends on remainder sets whose existence is guaranteed by the compactness property of the background logic. But since we do not have the compactness property to fall back upon, the contraction function we define will depend on a selection of complete consistent theories which will be intersected with the belief set $K$. Accordingly we assume a Choice Function (CF) $\delta: L \rightarrow 2^{T_{L}}$ that maps each formula $\varphi$ of $L$ to a set of complete theories $\delta(\varphi)$, subject to the following conditions:

1. $\delta(\varphi) \neq \emptyset$;

2. if $\varphi \notin C n(\emptyset)$, then $\delta(\varphi) \subseteq \bar{\omega}(\varphi)$

3. for any formulas $\varphi$ and $\psi$, if $\varphi \equiv \psi$ then $\delta(\varphi)=\delta(\psi)$. 
The purpose of the CF is to pick the best complete theories that do not entail a non-tautological formula $\varphi$ (condition 2). In the presence of a tautology, an agent keeps it body of knowledge untouched. In this case, the complete theories picked by a CF will simply be disregarded. For this reason, we impose no restriction over the complete theories a CF may select for a tautology. Condition 1 dictates that for any formula, at least one complete theory has to be selected. The last condition assures that a $\mathrm{CF}$ is not syntax-sensitive. The main difference between this choice function and the selection function used in the construction of the partial meet contraction is that while the latter picks remainders for a given theory (belief set) and formula pair, the former picks complete theories for a given formula, and the role of the belief set is postponed to the construction stage of the contracted set. In this sense, our approach better corresponds to the semantic counterpart of the AGM partial meet contraction function: $\llbracket K-\varphi \rrbracket=\llbracket K \rrbracket \cup \delta^{\prime}(\llbracket \neg \varphi \rrbracket)$ where $\delta^{\prime}$ is an appropriate choice function that picks models from an input set.

Definition 3.2. Let $K$ be a theory, $\varphi$ a formula, and $\delta$ a choice function. An operation ${ }_{-} \delta$ is an Exhaustive Contraction Function (ECF) iff

- $K{ }_{-\delta} \varphi=K \cap \bigcap \delta(\varphi)$, if $C n(\emptyset) \subset K \cap C n(\varphi)=C n(\varphi)$ and either $\neg \varphi \notin C n(\emptyset)$ or $\perp \in K$;

- $K{ }_{\delta} \varphi=K$, otherwise.

We explain the constraints imposed on the ECF by the above definition. A formula $\varphi$ must only be retracted from a belief set $K$ when: (1) $K$ is not simply the set of all the tautological formulas, (2) $\varphi$ is not a tautology and (3) $\varphi$ is in $K$. These constrains are jointly expressed as $C n(\emptyset) \subset K \cap C n(\varphi)=C n(\varphi)$. Besides, $\varphi$ has to be consistent, that is $\neg \varphi \notin C n(\emptyset)$, in order to be retracted from $K$; or $K$ is inconsistent. Otherwise, $K$ is left untouched.

For illustration purposes, let us contract a formula from a theory expressed in a non-compact logic. The Linear Temporal Logic (Clarke et al., 2001) is a good candidate for it. For simplicity, we will consider only two temporal operators of that logic: $G$ and $X$. The former means Globally (always) in the future, and $X$ means in the "neXt" time instant. We will keep the disjunction and negation of that logic which are interpreted classically. For our example, it will suffice to know two properties regarding these two operators. First, $C n\left(\left\{G p, p, X p, X^{2} p, \ldots, X^{n} p, \ldots\right\} \subseteq C n(G p)\right.$. In other words, $G p$ implies that $p$ is true in the current time instant and in all next future instants. As disjunction and negation are interpreted classically, we note that formulas such as $\neg X p \vee G p$ and $\neg \neg p$ also belong to $C n(G p)$. The second point we need to know is that $C n\left(\left\{p, X p, \ldots, X^{n} p, \ldots\right\}\right)=$ $C n(G p)$. For more details of this logic and its whole semantics, see (Clarke et al., 2001).

Example 7. Consider the theory $K=C n(G p)$ and we wish to contract Xp from it. Let our choice function be $\delta_{1}$ where:

1. If $C n(\psi)=C n(X p)$, then $\delta_{1}(\psi)=C n\left(\left\{p, \neg X p, X p \rightarrow G p, \neg X^{2} p,\left(X^{2} p\right) \rightarrow G p, \ldots\right\}\right) ;$

2. Else, if $C n(\psi)=C n(\emptyset)$ then $\delta_{1}(\psi)=T_{L}$;

3. Else, $\delta_{1}(\psi)=\left\{S \in T_{L} \mid \psi \notin S\right\}$. 
If $\psi$ is a tautology, we just let $\delta_{1}(\psi)=T_{L}$. The first constrain above regards the complete theories chosen to contract the formula Xp. As this is the only formula we are interested in retracting, for all other non-tautological formulas $\psi$ we let $\delta_{1}$ choose all the complete theories that do not imply $\psi$ (third constrain). So, $K-_{\delta_{1}} X p=K \cap \delta_{1}(X p)=C n\left(\left\{p, X p \rightarrow G p, X^{2} p \rightarrow G p, \ldots\right\}\right)$.

It is easy to notice that ${ }_{\delta_{1}}$ satisfies $\left(K_{1}^{-}\right)$to $\left(K_{4}^{-}\right)$. The extensionality postulate $\left(K_{6}^{-}\right)$follows from condition 3 of the definition of CF. For Recovery $\left(K_{5}^{-}\right)$, it suffices to note that $X p \rightarrow G p$ is in both $\delta_{1}(X p)$ and $K$, whereby, $\left(K-\delta_{1} X p\right)+X p=K$.

Now we want to prove our first representation result, jointly consisted of Theorems 3.3 and 3.4 below, that every ECF satisfies the six basic AGM contraction postulates, and conversely that the basic AGM contractions postulates characterise the ECF class.

Proposition 3.6. If a non-tautological formula $\varphi$ belongs to a theory $K$ then $C n(\emptyset) \subset K \cap C n(\varphi)=$ $C n(\varphi)$, and either $\varphi$ is consistent or $K$ is inconsistent.

At this point, we are ready to establish the first half of the representation result:

Theorem 3.3. Every ECF satisfies $\left(K_{1}^{-}\right)$to $\left(K_{6}^{-}\right)$.

Proof Sketch. Postulates $\left(K_{1}^{-}\right)$to $\left(K_{4}^{-}\right)$are easy to check. Postulate $\left(K_{6}^{-}\right)$is straightforward from the condition that a choice function cannot be syntax sensitive. So we focus on $\left(K_{5}^{-}\right)$. We need to show that $K \subseteq K \dot{-} \varphi+\varphi$. The case that $K \dot{-} \varphi=K$ is trivial, so we proceed to the case $K \dot{-} \varphi=K \cap \delta(\varphi)$. This means that $\varphi$ is not a tautology and is in $K$. So, $\llbracket K \rrbracket \subseteq \llbracket \varphi \rrbracket$ and $\delta(\varphi) \subseteq \bar{\omega}(\varphi)$. This implies that $\neg \varphi \in \bigcap \delta(\varphi)$. Therefore, $\llbracket K \rrbracket \cap \llbracket \bigcap \delta(\varphi) \rrbracket=\emptyset$. We then resort to the bijection between CISs and theories. We get $\llbracket K \dot{-} \varphi+\varphi \rrbracket=\llbracket K \dot{-} \varphi \rrbracket \cap \llbracket \varphi \rrbracket$, and $\llbracket K \dot{-} \varphi \rrbracket=\llbracket K \rrbracket \cup \llbracket \cap \varphi \rrbracket$. Substitution of the second in the first one gives us

$$
\llbracket K \dot{-} \varphi+\varphi \rrbracket=(\llbracket K \dot{-} \varphi \rrbracket \cap \llbracket \varphi \rrbracket) \cup(\llbracket \bigcap \delta(\varphi) \rrbracket \cap \llbracket \varphi \rrbracket) .
$$

As $\llbracket K \rrbracket \subseteq \llbracket \varphi \rrbracket$, we have that $\llbracket K \rrbracket \cap \llbracket \varphi \rrbracket=\llbracket K \rrbracket$. Moreover, $\llbracket \cap \delta(\varphi) \rrbracket$ has no models from $\varphi$ which means that $\llbracket \cap \delta(\varphi) \rrbracket \cap \llbracket \varphi \rrbracket=\emptyset$. Therefore, $\llbracket K \dot{-} \varphi+\varphi \rrbracket=\llbracket K \rrbracket$ which means that $K \dot{-} \varphi=K$. Thus $K \subseteq K \dot{-} \varphi+\varphi$.

The next step is to prove the second half of this representation result (Theorem 3.4), which consists in showing that every AGM rational contraction function is an ECF. One of the trickiest part will be to show that in some specific cases, precisely when a non-tautological formula $\varphi$ belongs to a theory $K$, a contraction $K \dot{-} \varphi$ can be indeed determined by intersecting $K$ with a set of complete theories (which is given by a choice function $\delta$ ). Precisely, the case: $K \dot{-} \varphi=K \cap \bigcap \delta(\varphi)$.

The first immediate task would be to find a way to determine the choice function $\delta$. Instead, we first propose to look at this task from another perspective, which shall then exhibit $\delta$ in a easy way. Notice that $\bigcap \delta(\varphi)$ is a theory, since $\delta(\varphi)$ is composed only by theories. So we can, initially, reduce our first task to find a theory, let us say $K_{Z}$ (which will play the role of $\bigcap \delta(\varphi)$ ) such that, $K \dot{-} \varphi=K \cap K_{Z}$. The left end of Figure 3.2 illustrates this idea. We have now two main issues to solve:

(i) how to find $K_{Z}$ ? and 


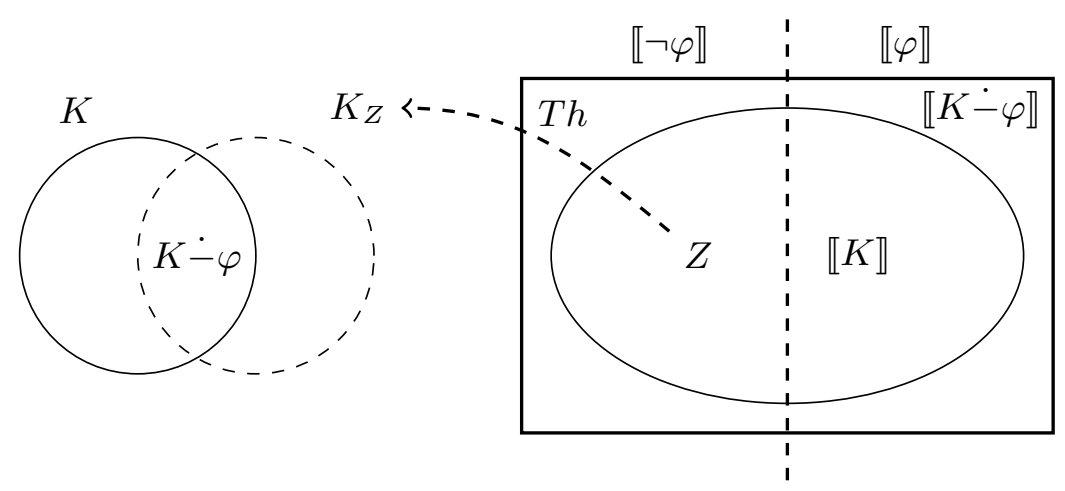

Figure 3.2: $A$ schema to find a theory $K_{Z}$ that can stand for $\bigcap \delta(\varphi)$ in the contraction $K \dot{-} \varphi=K \cap \bigcap \delta(\varphi)$.

(ii) how to find a set of complete theories whose intersection coincides with $K_{Z}$ ?

Determining $K_{Z}$ is not so easy as it may seem at a first glance. Mainly because there are two conditions to be satisfied: (1) $K \dot{-} \varphi=K \cap K_{Z}$, and (2) $\neg \varphi \in K_{Z}$. The second condition is not so straightforward and deserves some explanation. It is related to the fact of $\delta$ being a choice function, and to understand it we need to look back at the second condition of choice function definition which states that when $\varphi \in K$, the complete theories piked by $\delta$ must be only from $\omega(\neg \varphi)$, that is, $\delta(\varphi) \subseteq \omega(\neg \varphi)$. This implies that $\neg \varphi \in \bigcap \delta(\varphi)$ therefore in $\neg \varphi \in K_{Z}$.

To determine $K_{Z}$ we resort to the realm of CISs, and we explore the connection between theories and CISs and some properties that emerges from this connection regarding the intersection and union operations. We take the CIS $\llbracket K \dot{-} \varphi \rrbracket$ and the complement of it with the CIS of $K$, that is, we make $Z=\llbracket K \dot{-} \varphi \rrbracket \backslash \llbracket K \rrbracket$ (this is illustrated at the left hand of Figure 3.2). Then, we make $K_{Z}=T h(Z)$.

To see why it works, we need to look carefully at some properties we know about AGM contraction functions and the connection between theories and CISs that we have explored in the beginning of this chapter. First, we know that $K \dot{-} \varphi \subseteq K$ (due to the postulate ( $\mathrm{K}_{2}^{-}$) of inclusion), this implies that $K \subseteq \llbracket K \dot{-} \varphi \rrbracket$, as the function $\llbracket K \rrbracket$ is reverse-monotonic. The important point that follows from this is $\llbracket K \dot{-} \varphi \rrbracket=\llbracket K \rrbracket \cup Z$ (since $Z$ is the complement of $\llbracket K \dot{-} \varphi \rrbracket$ and $\llbracket K \rrbracket$ ). Recall from Proposition $3.5^{1}$ that $T h(\llbracket K \rrbracket \cup Z)=K \cap K_{Z}$ which means that $K \dot{-} \varphi=K \cap K_{Z}$. This corresponds to condition (1). The trickiest part is to prove that $\neg \varphi \in K_{Z}$. This can be achieved by showing that $Z \subseteq \llbracket \neg \varphi \rrbracket$ which is given in the following proposition (in which $K^{\prime}$ stands for $K \dot{-} \varphi$ and $Z$ for $\llbracket K \dot{-} \varphi \rrbracket \backslash \llbracket K \rrbracket)$.

Lemma 3.1. Let $K$ and $K^{\prime}$ be two theories such that $K^{\prime} \subseteq K$ and $\varphi$ a formula that it is in $K$ but not in $K^{\prime}$. If $K=K^{\prime}+\varphi$ then $\left(\llbracket K^{\prime} \rrbracket \backslash \llbracket K \rrbracket\right) \subseteq \llbracket \neg \varphi \rrbracket$.

Our results towards answering question (i) are summarized in the following observation.

Observation 3.6. Given an AGM rational function $\dot{-}$, a theory $K$ and a non-tautological formula $\varphi \in K$. Moreover, let $Z=\llbracket K \dot{-} \varphi \rrbracket \backslash \llbracket K \rrbracket$. The following are true,

1. $K \dot{-} \varphi=K \cap T h(Z)$ and

\footnotetext{
${ }^{1}$ Notice that application of Proposition 3.5 would require $Z$ to be a CIS. This is a technicality easy to solve which we leave to the proof at Appendix A.
} 
2. $\neg \varphi \in T h(Z)$.

Let us focus now on question (ii), and break it down into small pieces to make it easier to follow. First, why slice $K_{Z}$ into a set of complete theories? If we go back to the role of $K_{Z}$, we should recall that $K_{Z}$ is the theory that matches the intersection of the complete theories of $\delta(\varphi)$, that is, $K_{Z}=\bigcap \delta(\varphi)$. As our main task is indeed to determine who is $\delta$, splitting $K_{Z}$ in a set of complete theories would put us on the first path towards $\delta$. Precisely, if we find a set of consistent complete theories, let us say $A$, such that $K_{Z}=\bigcap A$, then such set is a candidate to $\delta(\varphi)$. In other words, we could make $\delta(\varphi)=A$. Basically, what we want is to decompose $K_{Z}$ in a collection of consistent complete theories $A$ (consistent because $\delta$ considers only consistent complete theories) such that $K^{\prime}$ can be reconstructed by intersecting the theories of $A$. Such a decomposition is given by the map dec $: 2^{L} \rightarrow 2^{T_{L}}$ defined as:

$$
\operatorname{dec}(K)=\left\{S \in T_{L} \mid K \subseteq S\right\}
$$

As expected, every theory $K$ can be reconstructed by intersecting the consistent complete theories given by $\operatorname{dec}(K)$.

Lemma 3.2. Let $K$ be a consistent theory, then $K=\bigcap \operatorname{dec}(K)$.

Now that we have the answers for questions (i) and (ii), it seems, at a first glance, that we have everything we need to construct a choice function $\delta$ for an AGM rational contraction function $\dot{-}$. It would suffice to make $\delta(\varphi)=\operatorname{dec}(T h(Z))$, where $Z$ stands for $\llbracket K \dot{-} \varphi \rrbracket \backslash \llbracket K \rrbracket$. This, however, satisfies only one of the conditions a choice function should obey (precisely condition 2), whereas condition (1), which states that $\delta(\varphi)$ cannot ever be empty, is not satisfied.

To see how this would violate condition (1), let us suppose that $K \dot{-} \varphi=K$. Then, we would get $Z=\llbracket K \dot{-} \varphi \rrbracket \backslash \llbracket K \rrbracket$ would give us $Z=\emptyset$. Following our naive strategy we then would make $\delta(\varphi)=\operatorname{dec}(\operatorname{Th}(Z))$, that is

$$
\delta(\varphi)=\operatorname{dec}(T h(\emptyset))=\operatorname{dec}(L)
$$

Notice, however, that $\operatorname{dec}(L)$ is empty, since $L$ is inconsistent and dec return only complete consistent theories. Therefore, $\delta(\varphi)=\emptyset$ violating, then, condition (1). This tell us that we need to deal accordingly with the case that $K \dot{-} \varphi=K$. The proposition below tell us exactly when this occurs: when either $\varphi$ is not in $K$ or $\varphi$ is a tautology.

Proposition 3.7. Let - be a function that satisfies $\left(K_{1}^{-}\right)$to $\left(K_{6}^{-}\right)$. Then, $K-\varphi \neq K$ iff $C n(\emptyset) \subset$ $K \cap C n(\varphi)=C n(\varphi)$, and either $\neg \varphi \notin C n(\emptyset)$ or $\perp \in K$.

A simple strategy then, is to construct $\delta$ in a piecewise way with two other conditions: make $\delta(\varphi)=T_{L}$ when $\varphi$ is a tautology; and $\delta(\varphi)=\bar{\omega}(\varphi)$, for when $\varphi \notin K$, which guarantees both condition (1) and (2). Condition (3) is trivially satisfied and simply states that a choice funciton is not syntax sensitive. The construction of $\delta$ is formalized in the following following proposition which states that $\delta$ is a choice function and that, as desired, intersecting $K$ with $\delta(\varphi)$ coincides with $K \dot{-} \varphi$. 


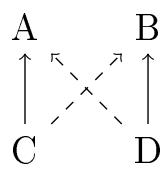

Figure 3.3: Mirroring. $A$ is preferred to $C$, hence to $D$.

Proposition 3.8. Let $\dot{-}$ be an $A G M$ rational contraction function, and $K$ a theory. Moreover, let $K^{\prime} \rrbracket K$ stand for $\llbracket K^{\prime} \rrbracket \backslash \llbracket K \rrbracket$. We construct the following function

$$
\delta(\varphi)=\left\{\begin{array}{cl}
T_{L} & \text { if } \varphi \in C n(\emptyset) \\
\operatorname{dec}(T h(K \dot{-} \varphi \rrbracket K) & \text { if } K \dot{-} \varphi \neq K \\
\bar{\omega}(\varphi) & \text { otherwise. }
\end{array}\right.
$$

The following are true,

1. $\delta$ is a choice function, and

2. if $\varphi \in K$ and $\varphi$ is not a tautology, then $K \dot{-} \varphi=K \cap \bigcap \delta(\varphi)$.

At this point we have all we need to our final representation result of this section.

Theorem 3.4. If a contraction function - satisfies the six basic contraction postulates, then there exists ECF $\dot{-}$ such that for any theory $K$ and formula $\varphi, K-\varphi=K \dot{-} \varphi$.

Proof. We need to show that there is a choice function $\delta$ such that

(i) $K \dot{-} \varphi=K \cap \delta(\varphi)$, if $C n(\emptyset) \subset K \cap C n(\varphi)=C n(\varphi)$, and either $\neg \varphi \notin C n(\emptyset)$ or $\perp \in K$

(ii) $K \dot{-} \varphi=K$, otherwise.

Item (ii) is straightforward from Proposition 3.7. So, we focus on item (i). Let us suppose that $C n(\emptyset) \subset K \cap C n(\varphi)=C n(\varphi)$, and either $\neg \varphi \notin C n(\emptyset)$ or $\perp \in K$. So $\varphi$ is not a tautology and $\varphi \in K$. Let $\delta$ be the choice function constructed according to the Proposition 3.8. So, we have that $K \dot{-} \varphi=K \cap \bigcap \delta(\varphi)$. Therefore, $\dot{-}$ is an ECF.

Our representation result for this section that involves the basic contraction postulates and exhaustive contraction functions immediately follows from Theorems 3.3 and 3.4.

\subsection{Construction: Full AGM Contraction Rationality}

In this section, we introduce a class of contraction functions that satisfy all eight AGM contraction postulates, the Blade Contraction. The main idea is to constrain the class of ECFs in a way that the choice function employed by each such ECF can be represented by a binary relation which respects two conditions, to be named $\bar{\omega}$-Maximal Cut and Mirroring.

Given a theory $K$ and a formula $\varphi$, a way to contract $K$ by $\varphi$ is to intersect $K$ with some consistent complete theories that do not imply $\varphi$. In a logic closed under classical negation, these consistent complete theories entail $\neg \varphi$. Recall that a consistent complete theory that does not imply 
a formula $\varphi$ is called a complement of $\varphi$, and the class of all complements of $\varphi$ is given by the set $\bar{\omega}(\varphi)=\left\{S \in T_{L} \mid \varphi \notin S\right\}$, and therefore $\bar{\omega}(\varphi)=\omega(\neg \varphi)$. We highlight that, due to the classical negation, $\bar{\omega}(\varphi \wedge \psi)=\bar{\omega}(\varphi) \cup \bar{\omega}(\psi)$. Also note that, since the logic is closed under classical negation, $\bar{\omega}(\varphi)$ is empty if and only if $\varphi$ is a tautology.

The basic idea is that the agent's choice function $\delta$ does not behave arbitrarily; its behaviour is "rationalised" by the agent's preference: the agent's preference is revealed by its choice behaviour. We represent this preference as a binary relation < over complements of $\varphi$. The two constraints we impose on $<$ are:

( $\bar{\omega}$-Maximal Cut) for every non-tautological formula $\varphi \in L, \bar{\omega}(\varphi)$ has a maximal element w.r.t. $<$;

(Mirroring) if $S_{1} \not \leq S_{2}$ and $S_{2} \not \leq S_{1}$; then for any $S^{\prime} \in T_{L}$, if $S_{1} \leq S^{\prime}$ then $S_{2} \leq S^{\prime}$.

The first condition on $<, \bar{\omega}$-maximal cut, is similar to the Limit Assumption of Lewis (1976) and the Finite Stopperedness of Gärdenfors and Makinson (1994). It guarantees that for every formula $\varphi$, an agent chooses at least one complement theory of $\varphi$. The purpose of the $\bar{\omega}$-Maximal cut is to ensure that every formula to be dropped will be successfully relinquished, that is, the theory being contracted will be intersected by complements of $\varphi$.

The second condition, Mirroring, is similar to the modular relation defined in (Meyer et al., 2000) which was based on modular partial orders of (Ginsberg, 1986) and (Lehmann and Magidor, 1992). Though the concept of modular relation is confined to be a partial order, we impose no such restriction. The intuition behind mirroring is that if an agent has no preference between two theories $A$ and $B$, then those that are preferable to $A$ should also be preferable to $B$ and vice versa. For instance, when dropping a formula $\varphi$ an agent may choose among the four complements of $\varphi$ : $A, B, C$ and $D$. It prefers $A$ to $C$ and $B$ to $D$, that is, its preference relation is $\{(C, A),(D, B)\}$ which is depicted in Figure 3.3 by solid arrows. So, it will choose both $A$ and $B$ to contract $\varphi$. However, there is no preference between $C$ and $D$. According to mirroring, all theories that are preferable to $C$ are also preferable to $D$ (and vice versa). Thus, the pairs $(C, B)$ and $(D, A)$ also need to be present in the relation (depicted by dashed arrows in Figure 3.3).

The rest of this section will proceed as follow. First, we introduce a relational choice function whose elements are picked according to a binary relation that satisfies maximal cut and show that contraction functions defined via such relational choice functions satisfy the postulate $\left(\mathrm{K}_{7}^{-}\right)$. Then we show that if the binary relation in question also satisfies mirroring, then the contraction functions defined via it satisfies both the postulates $\left(\mathrm{K}_{7}^{-}\right)$and $\left(\mathrm{K}_{8}^{-}\right)$.

From now on, unless otherwise specified, we consider only relations that satisfy $\bar{\omega}$-maximal cut. We will refer to it simply as maximal-cut, and we call a binary relation that satisfies maximal-cut contra-headed. First, we explain the idea of relational choice functions. Let $\delta$ be a choice function and $\varphi$ a formula. Moreover, let $<$ be a binary relation over the class of all complete theories. Recall that, given a set of complete theories $A, \max _{<}(A)$ is the set of all maximal theories in $A$ w.r.t. $<$. The choice function $\delta(\varphi)$ is restricted to pick only from the complement theories of $\varphi$, that is, $\delta(\varphi) \subseteq \bar{\omega}(\varphi)$. Thus, we will say that $\delta$ is relational if there is a binary relation $<$ over the set of all complete theories such that the elements picked by $\delta(\varphi)$ coincide with the maximal elements of 


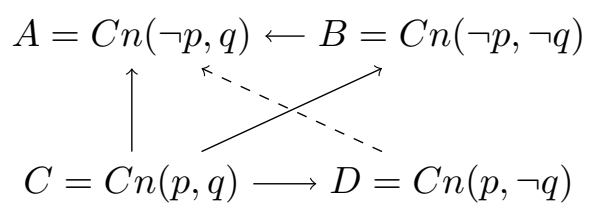

Figure 3.4: A depiction of Example 8.

$\bar{\omega}(\varphi)$ ordered by $<$, that is, $\delta(\varphi)=\max _{<}(\bar{\omega}(\varphi))$. We note that $\delta(\varphi)$ has to pick some complements of $\varphi$, for every non-tautological formula $\varphi$. In the case that $\delta$ is relational, it follows that there is a maximal element in $\bar{\omega}(\varphi)$ w.r.t. the corresponding binary relation $<$; that is, $<$ respects the maximal cut property, and so is contra-headed. A relational choice function whose corresponding binary relation is contra-headed will be called an annulment. We will use $\mu$ instead of $\delta$ to denote annulment functions.

Definition 3.3. Let $<$ be a contra-headed relation over $T_{L}$. An annulment is a function $\mu_{<}: L \rightarrow$ $2^{T_{L}}$ such that

$$
\mu_{<}(\varphi)= \begin{cases}\max _{<}(\bar{\omega}(\varphi)) & \text { if } C n(\varphi) \neq C n(\emptyset) \\ \text { some } \emptyset \neq X \subseteq T_{L} & \text { otherwise }\end{cases}
$$

Note that, for the tautologies, an annulment is free to chose any element, since tautologies do not have any complement. As expected an annulment is indeed a choice function.

Lemma 3.3. Every annulment is a choice function.

Now we proceed to define a new contraction function that we call the Blade Contraction Function $(B C F)$. The idea is similar to an ECF, we simply restrict the choice function to be an annulment function. This will ensure that every BCF satisfies the postulate $\left(\mathrm{K}_{7}^{-}\right)$.

Definition 3.4. Let $\mu_{<}$be an annulment w.r.t. a contra-headed relation $<$. A Blade Contraction Function $(B C F) \dot{-}<$ is constructed from $<$ as follows:

1. $K \dot{-}_{<} \varphi=K \cap \bigcap \mu_{<}(\varphi)$, if both

(i) $C n(\emptyset) \subset K \cap C n(\varphi)=C n(\varphi)$, and

(ii) either $\neg \varphi \notin C n(\emptyset)$ or $\perp \in K$;

2. $K \dot{-}_{<} \varphi=K$, otherwise.

Notice that the definitions of BCF and ECF are quite similar, the only difference is that a BCF involves an annulment, and an ECF involves a choice function. It trivially follows from Definition 3.4 and Lemma 3.3 that:

Corollary 3.5. Every Blade Contraction Function is an Exhaustive Contraction Function.

Example 8. Let $K=C n(p, q)$ be a propositional theory, and $<$ the binary relation depicted by solid arrows in Figure 3.4. Moreover, let $\mu_{<}$be the respective annulment function. The complements of $p, q$ and $p \wedge q$ are respectively $\bar{\omega}(p)=\{A, B\}, \bar{\omega}(q)=\{B, D\}$ and $\bar{\omega}(p \wedge q)=\{A, B, D\}$. According to $<$ which is contra-headed: $\mu_{<}(p)=\{A\}, \mu_{<}(q)=\{B, D\}$ and to $\mu_{<}(p \wedge q)=\{A, D\}$. Thus we have: 


$$
\begin{array}{lll}
K \dot{-}_{<p} & =K \cap \bigcap \mu(p) & =C n(q) \\
K \dot{-}_{<q} & =K \cap \bigcap \mu_{<}(q) & =C n(\neg q \vee p) \\
K \dot{-}_{<p} \wedge q & =K \cap \bigcap \mu_{<}(p \wedge q) & =C n(p \vee q)
\end{array}
$$

A blade contraction behaves similarly to an ECF, the difference is that the choice function picks the complements of a formula $\varphi$ according to a binary relation. The binary relation guarantees that a BCF satisfies postulate $\left(\mathrm{K}_{7}^{-}\right)$.

Theorem 3.5. Every BCF satisfies $\left(K_{1}^{-}\right)$to $\left(K_{7}^{-}\right)$.

Though blade contractions satisfy postulate $\left(\mathrm{K}_{7}^{-}\right)$, maximal cut alone is not enough to satisfy postulate $\left(\mathrm{K}_{8}^{-}\right)$. This can be seen on the blade function of the Example 8. First, from the example we have that $K \dot{-}{ }_{<} q=C n(\neg q \vee p)$ and $K \dot{-}{ }_{<} p \wedge q=C n(p \vee q)$. Now, notice that $q \notin K \dot{-}<p \wedge q$ and that $C n(p \vee q) \nsubseteq C n(\neg q \vee p)$ which implies that $K \dot{-}_{<} p \wedge q \nsubseteq K \dot{-}_{<} q$. This means that $\dot{-}_{<}$does not satisfy postulate $\left(\mathrm{K}_{8}^{-}\right)$.

To capture $\left(\mathrm{K}_{8}^{-}\right)$it will suffice to consider contra-headed relations that also satisfy mirroring. Lemma 3.6 will help explain how postulates $\left(\mathrm{K}_{7}^{-}\right)$and $\left(\mathrm{K}_{8}^{-}\right)$dictate the way complements of a conjunction $\varphi \wedge \psi$ are chosen in an ECF. To understand why mirroring is related to postulate $\left(\mathrm{K}_{8}^{-}\right)$, we will need the condition $\mathrm{C} 2$ of that lemma.

According to condition $\mathrm{C} 2$ of Lemma 3.6, if some complement $A$ of a formula $\varphi$ is chosen to contract a conjunction $\varphi \wedge \psi$, then all theories chosen to contract $\varphi$ must also be picked to contract the conjunction $\varphi \wedge \psi$. Another way of reading this is: if a complement $K^{\prime}$ of $\varphi$ is not chosen in the contraction of $\varphi$, the complements of $\varphi$ that were not chosen for the contraction of this formula are not chosen in the contraction of $\varphi \wedge \psi$. In terms of a relation, this is equivalent to say that if a complement is not maximal in $\bar{\omega}(\varphi)$ then it is also not maximal in $\bar{\omega}(\varphi \wedge \psi)$.

To see how mirroring guarantees condition $\mathrm{C} 2$, let us first look at the contraction function of Example 8. Let $\mu_{<}$be the annulment function of that example. We see that though the theory $C n(\neg p \wedge q)$ belongs both to $\mu_{<}(p)$ and $\mu_{<}(p \wedge q)$, the theory $C n(p \wedge \neg q)$ is not in $\mu_{<}(p \wedge q)$. So, let us see what modifications are necessary in order to turn $<$ into a relation that satisfies $\mathrm{C} 2$. First, we notice that the only reason why $<$ does not satisfy mirroring is because though $C n(p, \neg q)$ and $C n(\neg p \wedge \neg q)$ are not comparable, $C n(p, \neg q)$ is not less preferable than $C n(\neg p, q)$. Note that this is the same reason why $\mu_{<}$does not satisfy $\mathrm{C} 2$ : though $C n(p, \neg q)$ and $C n(\neg p \wedge \neg q)$ are chosen to contract q, only $C n(\neg p \wedge \neg q)$ was chosen to contract $p \wedge q$. As $C n(p \wedge \neg q)$ is already less preferable than $C n(\neg p \wedge q)$, the theory $C n(p \wedge \neg q)$ cannot be chosen to contract $p \wedge q$. So, an option to make it satisfy $\mathrm{C} 2$ is to make $C n(p \wedge \neg q)$ also less preferable then $C n(\neg p \wedge q)$. This new relation $<_{1}$ is depicted in Figure 3.4 as arrows. So we have that,

$$
\begin{aligned}
& K \dot{-}_{<1} p \quad=K \cap C n(\neg p \wedge q) \quad=C n(q) \\
& K \dot{-}_{<1} q \quad=K \cap \bigcap \mu_{<}(q) \quad=C n(\neg q \vee p) \\
& K \dot{-}_{<1} p \wedge q=K \cap C n(\neg p \wedge q) \quad=C n(q)
\end{aligned}
$$

It is easy to see that the $\dot{-}_{<1}$ satisfies $\mathrm{C} 2$ and also $\left(\mathrm{K}_{8}^{-}\right)$.

Now we proceed to prove that every Blade Contraction function whose annulment respects mirroring satisfies postulate $\left(\mathrm{K}_{8}^{-}\right)$. This claim is proven as Theorem 3.6 which brings us to Lemma 3.4 and Lemma 3.5. 
Lemma 3.4. Let $A$ and $B$ be two sets ordered by a contra-headed relation $<$ that satisfies mirroring. If some maximal element of $A$ is also a maximal element of $A \cup B$, then $\max _{<}(A) \subseteq \max _{<}(A \cup B)$.

Lemma 3.5. Let $\mu_{<}$be an annulment. Every complete theory $S$ in $\mu_{<}(\varphi \wedge \psi)$ is such that either $S \in \mu_{<}(\varphi)$ or $S \in \mu_{<}(\psi)$.

Theorem 3.6. Let $\dot{-}$ be a $B C F$ w.r.t. an annulment $\mu_{<}$. If $<$satisfies mirroring, then $\dot{-}$ satisfies $\left(K_{8}^{-}\right)$.

So, from Theorem 3.5 and Theorem 3.6, we conclude that every BCF, whose annulment is based on a relation satisfying mirroring, satisfies $\left(\mathrm{K}_{7}^{-}\right)$and $\left.\left(\mathrm{K}_{8}^{-}\right)\right)$.

The next step is to prove that every contraction function that satisfies all eight AGM contraction postulates is a BCF. First we recall that every function that satisfies the six basic AGM postulates is an ECF (Theorem 3.4), whereby every function that satisfy the eight AGM postulates is also an ECF. Now, it will be sufficient to show that for every ECF $\dot{-}_{\delta}$ that satisfies $\left(\mathrm{K}_{7}^{-}\right)$and $\left(\mathrm{K}_{8}^{-}\right)$, the choice function $\delta$ is in fact an annulment, that is, $\delta(\varphi)=\max _{<}(\bar{\omega}(\varphi))$ for some binary relation $<$ that satisfies mirroring. This is a bit tricky. Note that we are considering only contra-headed binary relations. From here onwards, unless stated otherwise, any choice function mentioned is taken to concern an ECF that satisfies all eight AGM postulates. We will need to show how to construct a contra-headed relation for a choice function.

We start from a basic idea. Given a choice function $\delta$, and a formula $\varphi$ we will need a relation $<_{\varphi}$ such that the maximal elements of $\bar{\omega}(\varphi)$ ordered via $<_{\varphi}$ coincide with $\delta(\varphi)$, that is, $\delta(\varphi)=$ $\max _{<\varphi}(\bar{\omega}(\varphi))$. This is easy, and it suffices to make $<_{\varphi}=\{(A, B) \in \bar{\omega}(\varphi) \times \bar{\omega}(\varphi) \mid A \notin \delta(\varphi)$ and $B \in$ $\delta(\varphi)\}$

The tricky bit is to construct the general relation for the whole class of complete theories, because simply compiling every $<_{\varphi}$ together as a relation $<=\bigcup_{\varphi \in L}<_{\varphi}$ will not satisfy mirroring. To notice that, let us take an example. Let us take a choice function $\delta$, such that for a formula $\varphi$, it chooses a complete theory $A$ among the set of complete theories $A, B$ and $C$, whereas for a formula $\psi$ it chooses the theory $D$ over the set of complete theories $D, E$ and $F$. Moreover, for the conjunction $\varphi \wedge \psi$ it chooses only $D$. Summarizing, we have

$$
\begin{aligned}
\delta(\varphi) & =\{A\} \subset \bar{\omega}(\varphi)=\{A, B, C\} \\
\delta(\psi) & =\{D\} \subset \bar{\omega}(\psi)=\{D, E, F\} \\
\delta(\varphi \wedge \psi) & =\{D\} \subset \bar{\omega}(\varphi \wedge \psi)=\{A, B, C, D, E, F\} .
\end{aligned}
$$

The relations $<_{\varphi},<_{\psi}$ and $<_{\varphi \wedge \psi}$ are illustrated in Figure 3.5. The relation $<_{\varphi \wedge \psi}$ consists of all solid arrows in the figure, whereas the relations $<_{\varphi},<_{\psi}$ are depicted by the arrows inside its respective spheres. It is easy to see that $<_{\varphi \wedge \psi}$ is a contra-headed relation for $\delta$. This relation, however, does not satisfy mirroring: observe that $B \nless E$ and $E \nless B$, however $E<D$ and $B \nless D$ violating mirroring. Hence more is needed. This example suggests that if we relate all the non maximal elements of $<_{\varphi}$ to the maximal elements of $<_{\psi}$, that is, add the pairs $(B, D)$ and $(C, D)$, the resulting relation does satisfy mirroring. This operation that relates the non-maximal elements of a relation $<_{\varphi}$ to the maximal ones of another relation $<_{\psi}$ will be called a triangulation. 


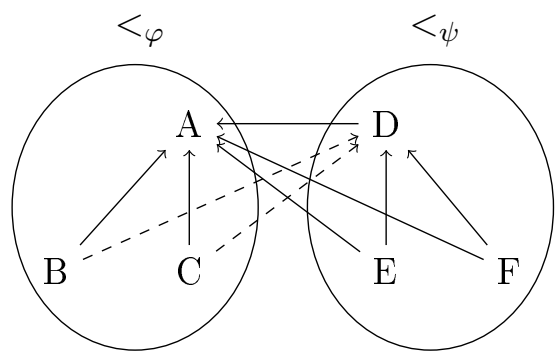

Figure 3.5: Three relations: $<_{\varphi},<_{\psi}$ and $<_{\varphi \wedge \psi}$.

Definition 3.5 (Triangulation). Let $\delta$ be a choice function, $\varphi$ and $\psi$ be two formulas. The triangulation of $\varphi$ and $\psi$ w.r.t. $\delta$ is the relation $\nabla(\varphi, \psi)=\{(A, B) \in \bar{\omega}(\varphi) \times \bar{\omega}(\psi) \mid A \in \bar{\omega}(\varphi) \backslash(\delta(\varphi) \cup$ $\bar{\omega}(\psi))$, and $B \in \delta(\psi)\}$.

For instance, the triangulation for $\varphi$ and $\psi$, in the example of Figure 3.5, is $\nabla(\varphi, \psi)=\{(B, D),(C, D)\}$ which is depicted by dashed arrows in that same figure.

Our intention is to show that every ECF that satisfies $\left(\mathrm{K}_{7}^{-}\right)$and $\left(\mathrm{K}_{8}^{-}\right)$is a blade contraction. Hence we need to show that there exists a binary relation $<$ satisfying mirroring such that $\delta(\varphi)=$ $\max _{<}(\bar{\omega}(\varphi))$, that is, $\delta$ is an annulment. Employing triangulation, we can construct such a relation that we will call the shadow of $\delta$.

Definition 3.6. Let $\delta$ be a choice function. Then the shadow of $\delta$ is a relation $<\subseteq T_{L} \times T_{L}$ such that $(A, B)$ is in $<$ iff, for some formulas $\varphi$ and $\psi$ :

$$
\begin{aligned}
& \text { either }(A, B) \in\left(\bigcup_{\varphi \in L}<_{\varphi}\right) \\
& \text { or }(A, B) \in \nabla(\varphi, \psi) \cup \nabla(\psi, \varphi) .
\end{aligned}
$$

In the definition above, the first condition compiles all the relations $<_{\varphi}$ together, while the second one employs the triangulation. To show that an ECF $\dot{-}_{\delta}$ is an annulment, we will need more then its shadow. The tricky part is to show how the choice function $\delta$ behaves as an annulment. For this, we will explore an interesting class of choice functions: the saturated $K$-grounded choice functions. These functions will provide us with the last properties we need for the representation theorem.

Definition 3.7. Given a theory $K$, a choice function $\delta$ is a saturated $K$-grounded if and only if it satisfies the two following conditions:

Saturation: $\delta(\varphi)=\operatorname{dec}(\bigcap \delta(\varphi)$, if $\varphi$ is not a tautology;

K-ground: $\delta(\varphi)=\operatorname{dec}(K) \cap \bar{\omega}(\varphi)$, if $\varphi \notin K$.

We explain the intuition behind the saturated $K$-grounded choice functions. We start by the saturation condition. For this, we first need to recall how choice functions help in devising contraction functions. An ECF $\dot{-}_{\delta}$ only resorts to a choice function when a non-tautological formula $\varphi$ belongs to a theory $K$, and the contraction boils down to $K \dot{-} \varphi=K \cap \bigcap \delta(\varphi)$. We can look at it from the following perspective: the role of $\delta$ is to provide a theory $K^{\prime}$ to be intersected with 
$K$. However, instead of given $K^{\prime}$ explicitly it gives a set of complete theories whose intersection corresponds to the desired theory $K^{\prime}$, that is, $K^{\prime}=\bigcap \delta(\varphi)$. From this perceptive, a saturated choice function behaves in a strict way picking all the complete consistent theory that contains the theory $K^{\prime}$, that is, $\delta(\varphi)=\operatorname{dec}\left(K^{\prime}\right)$.

On the another side, when a formula $\varphi$ is not in $K$, a choice function is not consulted. In this case, the $K$-grounded condition impose that a choice function should pick only the complete consistent theories of $K$ that do not entails $\varphi$, that is, the complements of $\varphi$, which is given by $\bar{\omega}(\varphi)$, within $\operatorname{dec}(K)$.

An interesting property is that every choice function $\delta$ can be swapped by a saturated Kgrounded choice function $\delta^{\prime}$ without inflicting any damage to $\dot{-}_{\delta}$. In other words, $\dot{-}_{\delta}$ is equivalent to $\dot{-}_{\delta^{\prime}}$, as pointed on Observation 3.7 below.

Observation 3.7. Let $K$ be a theory, and $\dot{-}_{\delta}$ be an ECF, there is a saturated $K$-grounded choice function $\delta^{\prime}$ such that $K \dot{-}_{\delta} \varphi=K \dot{-}_{\delta^{\prime}} \varphi$, for every formula $\varphi$.

For now on, unless otherwise specified, we will assume that a K-grounded choice function is also saturated. The next step is to show that every $K$-grounded choice function $\delta$ is indeed an annulment. This involves showing that $\delta(\varphi)=\max _{<}(\bar{\omega}(\varphi))$, for some contra-headed relation $<$. Such a relation, as we shall see, is the shadow of $\delta$.

To achieve the representation theorem, we also need to show that the shadow also satisfies mirroring. Theorem 3.7 shows that every $K$-grounded choice function from an ECF that satisfies $\left(\mathrm{K}_{7}^{-}\right)$and $\left(\mathrm{K}_{8}^{-}\right)$is an annulment, and Theorem 3.8 shows that the shadow of $\delta$ satisfies mirroring.

Lemmas 3.6, 3.7 and Corollary 3.6 below are of assistance to prove Theorem 3.7. Lemma 3.6 correlates how a choice function $\delta$ behaves in the presence of the two supplementary postulates. Given two formulas $\varphi$ and $\psi$, according to $\left(\mathrm{K}_{7}^{-}\right)$, in order to contract the conjunction of the formulas $\varphi$ and $\psi$, the function $\delta$ can choose only from the complete theories picked to individually contract $\varphi$ and $\psi$, that is, from $\delta(\varphi) \cup \delta(\psi)$. For the postulate $\left(\mathrm{K}_{8}^{-}\right)$, if some complete theory picked to contract $\varphi \wedge \psi$ was also picked to contract $\varphi$, then all complete theories chosen to contract $\varphi$ must also be picked to contract the conjunction $\varphi \wedge \psi$. One immediate consequence of these two conditions, as shown in Corollary 3.6, is that if a complete theory is chosen either to contract $\varphi$ or to contract $\psi$, then the set of complete theories picked to contract the conjunction $\varphi \wedge \psi$ is precisely the complete theories chosen to contract both $\varphi$ together with those picked to contract $\psi$, that is, the set $\delta(\varphi) \cup \delta(\psi)$. Lemma 3.7 exhibits a similar property of Corollary 3.6 but in terms of the shadow relation of $\delta$. It states that the maximal elements of $<_{\varphi}$ and $<_{\psi}$ are also maximal elements of $<_{\varphi \wedge \psi}$, when at least one theory from the intersection of $\delta(\varphi) \cap \delta(\psi)$ is chosen. This is because the elements chosen by each $\delta(\varphi)$ are preserved as maximal elements of the shadow of $\delta$.

Lemma 3.6. Let $\dot{-}_{\delta}$ be an $E C F$, and $\varphi$ and $\psi$ be two non-tautological formulas. If $\delta$ is K-grounded, then

(C1) If $\dot{-}_{\delta}$ satisfies $\left(K_{7}^{-}\right)$, then $\delta(\varphi \wedge \psi) \subseteq \delta(\varphi) \cup \delta(\psi)$;

(C2) If $\dot{-}_{\delta}$ satisfies $\left(K_{8}^{-}\right)$, then $\delta(\varphi) \subseteq \delta(\varphi \wedge \psi)$, given there is some theory $S$ of $\delta(\varphi)$ in $\delta(\varphi \wedge \psi)$. 
Corollary 3.6. Let $\dot{-}_{\delta}$ be an ECF that satisfies $\left(K_{7}^{-}\right)$and $\left(K_{8}^{-}\right)$, and $\varphi$ and $\psi$ be two non-tautological formulae. If $\delta$ is K-grounded and saturated, and $\delta(\varphi) \cap \delta(\psi) \neq \emptyset$ then $\delta(\varphi \wedge \psi)=\delta(\varphi) \cup \delta(\psi)$.

Proposition 3.9. Let $\dot{-}_{\delta}$ be an ECF, $\delta$ be K-grounded, and $\varphi$ and $\psi$ be two non-tautological formulas. If $C \notin \delta(\varphi), C \in \delta(\psi)$ and $C \in \delta(\varphi \wedge \psi)$, then $\dot{-}_{\delta}$ does not satisfy (K$\left.K_{7}^{-}\right)$or ( $\left.K_{8}^{-}\right)$.

Lemma 3.7. Given an $E C F \dot{-}_{\delta}$ satisfying $\left(K_{7}^{-}\right)$and $\left(K_{8}^{-}\right)$, and non-tautological formulas $\varphi$ and $\psi$ such that $\delta$ is K-grounded. If $\delta(\varphi) \cap \delta(\psi) \neq \emptyset$ then $<_{\varphi} \cup<_{\psi} \subseteq<_{\varphi \wedge \psi}$.

Theorem 3.7. If an ECF $\dot{-}_{\delta}$ satisfies $\left(K_{7}^{-}\right)$and $\left(K_{8}^{-}\right)$, and $\delta$ is $K$-grounded, then the choice function $\delta$ is an annulment.

So far, we have shown that every function that satisfies all the eight postulates is an ECF whose choice function is an annulment, that is, a Blade Contraction function. The only other thing we need to finish off the representation result is to show that the shadow of such a choice function respects mirroring.

Theorem 3.8. Let $\dot{-}_{\delta}$ be an ECF that satisfies $\left(K_{7}^{-}\right)$and $\left(K_{8}^{-}\right)$. If $\delta$ is $K$-grounded, then the shadow $<$ of $\delta$ satisfies mirroring.

From Theorem 3.5 and Theorem 3.6, together with Theorem 3.8, the desired representation theorem between the eight AGM contraction postulates and the Blade Contraction function class easily follows:

Theorem 3.9. Every contraction function that satisfies all the eight postulates is equivalent to a $B C F$.

\subsection{AGM Revision Rationality}

In the previous sections most was said about AGM contraction rationality: we have shown that Tarskian logics closed under classical negation and disjunction are AGM-compliant, and we devised contraction functions characterized by both basic postulates and supplementary postulates. This section is devoted for the another branch of AGM theory: the AGM revision.

In the presence of the AGM assumptions, the Grove's system of spheres (Grove, 1988) yields (fully) AGM rational revision functions. The main idea of this system is that an agent considers a preference relation < over all complete theories (called spheres), and chooses the best spheres (modulo $<$ ) to perform the revision. In a semantic approach, the spheres can be seen as the interpretations (or worlds) of the underlying logic. The preference relation dictates an ordering of proximity among the worlds of the underlying logic. The revision output is the theory satisfied by the closest worlds of $\varphi$ that intersects the words of $K$. Though the system of spheres yields fully AGM rational revision functions, the representation result between these functions and the AGM revision postulates relies strongly on compactness. Therefore, we propose new classes of AGM rational revision functions that have no dependency on compactness.

We first devise in Section 3.4.1 a class of functions for the basic AGM revision postulates, and then in the Section 3.4.2 we extend such class of functions to cover the supplementary postulates. As in the AGM contraction branch, the AGM revision supplementary postulates will be captured by a binary relation over complete consistent theories. 


\subsubsection{Exhaustive Revision Function: AGM basic rationality}

The idea to construct a revision function that captures the six first AGM postulates is similar to the the intuition behind an ECF: in the light of a formula $\varphi$, some complete consistent theories will be chosen, and the intersection of these selected theories will correspond to the revision output. To decide which of these consistent complete theories should be chosen, we will resort to a choice function whose role intuitively is to pick consistent complete theories deemed the best ones for the revision. Unlike an Exhaustive Contraction Function, which relies on a choice function to select only complete theories that do not entail a formula $\varphi$, for revision we will impose that a choice function selects only complete theories that do entail a formula $\varphi$. This difference is because as a contraction function wants to expel a formula $\varphi$, it looks for theories whose intersection does not entail $\varphi$; on the other hand, as a revision function wants to incorporate $\varphi$, it asks a choice function to look for consistent complete theories that do entail $\varphi$. Recall that the set of all consistent complete theories that entail a formula $\varphi$ is given by $\omega(\varphi)$. To avoid misinterpretation, a "choice" function $\delta: L \rightarrow 2^{T_{L}}$ for revision purposes will be called a casting function subject of the following constraints:

(1) $\delta(\varphi) \neq \emptyset$

(2) if $C n(\varphi) \neq C n(\perp)$, then $\delta(\varphi) \subseteq \omega(\varphi)$;

(3) for every formulas $\varphi$ and $\psi$, if $\varphi \equiv \psi$ then $\delta(\varphi)=\delta(\psi)$.

The constraints put upon a casting function are similar to the constraints of a choice function. The only difference is condition (2) which requires the casting function to choose complete theories that entail a formula $\varphi$.We highlight that as a casting function $\delta$ can choose only among consistent complete theories, the restriction to pick complete consistent theories that entail a formula $\varphi$ only occurs when $\varphi$ is consistent. In the case that $\varphi$ is inconsistent, $\delta$ is free to choose whatever it wants to. Conditions (1) and (3) are respectively the same as in a choice function definition, and no further explanation is required.

With the casting functions in hand, we can proceed to define a class of revision functions that satisfy the AGM basic postulates which we shall call Exhaustive Revision Function (ERF). The intuition behind an ERF is that given a theory $K$ and a formula $\varphi$ : if $\varphi$ is inconsistent, or expanding $\mathrm{K}$ with $\varphi$ does not lead to a inconsistent theory (this corresponds to K being consistent with $\varphi$ ), then such an expansion is the revision result. On the other hand, if $\varphi$ is inconsistent with $K$, then the revision function can choose any theory that entails $\varphi$. Such a theory is constructed by the intersection of the theories provided by a casting function.

Definition 3.8. Let $K$ be a theory, $\varphi$ a formula and $\delta$ a casting function. An Exhaustive Revision Function $(E R F) *_{\delta}$ is defined as

$$
K *_{\delta} \varphi= \begin{cases}K+\varphi & \text { if } K+\varphi \neq C n(\perp) \text { or } C n(\varphi)=C n(\perp) \\ \bigcap \delta(\varphi) & \text { otherwise }\end{cases}
$$

For illustration purposes, let us make use of an ERF on a non-compact logic: the LTL which we also have used in Section 3.2 to illustrate the ECFs. We recall that at Example 7, we have used two temporal operators of this logic: $X$ and $G$ which means respectively "neXt" time instant and 
"Globally" (always) in the future. Now, we will make use of another temporal operator of LTL: the $F$ operator which means "eventually". For our purpose, it will suffice to know three properties of this operator: $F \neg p$ is consistent, whereas $C n(G p)+F \neg p$ is inconsistent, and $F \neg p \in C n(X \neg p)$. The idea behind the last property is that if in the next time instant $\neg p$ occurs, then $\neg p$ eventually occurs.

Example 9. Consider the theory $K=C n(G p)$ and we wish to revise it by $F \neg p$. Let our casting function be:

1. $\delta_{2}(F \neg p)=\left\{C n\left(\left\{p, X \neg p, F \neg p, X^{2} \neg p, \ldots\right\}\right)\right\} ;$

2. Else, if $C n(\varphi)=C n(\perp)$ then $\delta_{2}(\varphi)=T_{L}$;

3. Else, $\delta_{2}(\varphi)=\omega(\varphi)$.

If $\varphi$ is an inconsistent formula, then we just make $\delta_{2}(\varphi)=T_{L}$. The first constraint of $\delta_{2}$ above regards the consistent complete theories chosen to revise the formula $F \neg p$. As this is the only formula we have interest to make a revision, for all other formulae $\psi$ we just take all the complete consistent theories that entail $\psi$ (condition 3 above).

As $C n(G p)+F \neg p$ is inconsistent and $F \neg p$ is consistent, we get that

$$
\begin{aligned}
K *_{\delta 2} F \neg p & =\bigcap\left\{C n\left(\left\{p, X \neg p, F \neg p, X^{2} \neg p, \ldots\right\}\right)\right\} \\
& =C n\left(\left\{p, X \neg p, F \neg p, X^{2} p, \ldots\right\}\right) .
\end{aligned}
$$

Clearly, $K *_{\delta 2} F \neg p$ is a theory, and $F \neg p \in K *_{\delta 2} F \neg p$. This means that ( $\left.K_{1}^{*}\right)$ and ( $\left.K_{2}^{*}\right)$ are satisfied. It is easy to check that $*_{\delta 2}$ satisfies $\left(K_{3}^{*}\right)$ to $\left(K_{5}^{*}\right)$. Postulate ( $\left.K_{6}^{*}\right)$ follows directly from condition (3) of the definition of a casting function.

Having seen how an ERF works, we can proceed to show the representation theorem between the AGM postulates and the ERFs. Our first step toward the representation theorem is given on Theorem 3.10 and 3.11 below that state respectively that every ERF satisfies the AGM basic postulates and conversely that every function that satisfies the basic AGM revision postulates is an ERF.

Theorem 3.10. Every ERF satisfies $\left(K_{1}^{*}\right)-\left(K_{6}^{*}\right)$.

The next step toward our first representation result is to show that every rational AGM revision function is equivalent to some ERF. First, according to the ERF definition, $K * \varphi=K+\varphi$ when either $\varphi$ is inconsistent or $K+\varphi$ is consistent. It turns out that this feature is inherent not only to ERFs, but for every AGM rational revision function:

Proposition 3.10. If a revision function $*$ satisfies $\left(K_{1}^{*}\right)-\left(K_{6}^{*}\right)$, then $K+\varphi \neq C n(\perp)$ or $C n(\varphi)=$ $C n(\perp)$ iff $K * \varphi=K+\varphi$.

The second piece of the puzzle regards the other condition of the ERF definition, that is, when $K * \varphi=\bigcap \delta(\varphi)$. This tells us we need to construct a casting function $\delta$ whose intersection of the complete theories cast for a formula $\varphi$ results in $K * \varphi$. In summary, $K * \varphi=\bigcap \delta(\varphi)$ when 
$K * \varphi \neq K+\varphi$. If we get to construct such a casting function, then we will have all the missing pieces to solve the puzzle. We can start by looking for a way of splitting every theory $K$ in terms of a collection $A$ of consistent complete theories, and then reconstruct $K$ by intersecting all the theories in $A$. Such a decomposition is given by the function dec $: 2_{L} \rightarrow 2^{T_{L}}$ we defined in Section 3.2. For convenience, we recall its definition here:

$$
\operatorname{dec}(K)=\left\{S \in T_{L} \mid K \subseteq S\right\}
$$

The decomposition function dec will be used as a foundation to construct the so desired casting function for the case that $K * \varphi \neq K+\varphi$. Recall from Lemma 3.2 that, as expected, every theory $K$ can be reconstructed by intersecting the consistent complete theories given by $\operatorname{dec}(K)$.

Now we have all we need to achieve the last piece of the representation result:

Theorem 3.11. If a revision function satisfies the six basic postulates, then it is equivalent to some ERV.

Proof Sketch: Given a theory $K$ and a revision function $*$, we need to show that for some $E R F *_{\delta}, K * \varphi=K *_{\delta} \varphi$, for every formula $\varphi$. From proposition 3.10, we already have that both $K * \varphi$ and $K *_{\delta} \varphi$ results in $K+\varphi$, when either $C n(\varphi)=C n(\perp)$ or $K+\varphi=C n(\perp)$. Therefore, we just need to proceed to the case that $\varphi$ is consistent and both $K * \varphi$ and $K *_{\delta} \varphi$ are different of $K+\varphi$. In this case, we need to show that $K * \varphi=K *_{\delta} \varphi=\bigcap \delta(\varphi)$. Putting in a simple way, we have to show that it is possible to decompose $K * \varphi$ in terms of a collection $A$ (given by a decomposition function $f(\varphi)$ ) of complete theories and then reconstruct $K * \varphi$ by intersecting all the complete consistent theories in $A$ (i.e. $\bigcap f(\varphi)$ ). We already know that this can be done via the decomposition function dec. Furthermore, the reconstruction process is guaranteed by Lemma 3.2. From dec we can construct a casting function $\delta$ as follows

$$
\delta(\varphi)=\left\{\begin{array}{cl}
\operatorname{dec}(K * \varphi) & \text { if } C n(\varphi) \neq C n(\perp) \\
T_{L} & \text { otherwise. }
\end{array}\right.
$$

It is easy to check that $\delta$ is a casting function. The only thing left to show is that $K * \varphi=\bigcap \delta(\varphi)$. Recall that we are dealing with the case that $C n(\varphi) \neq C n(\perp)$. Thus, $\delta(\varphi)=\operatorname{dec}(K * \varphi)$ which implies that $\bigcap \delta(\varphi)=\bigcap \operatorname{dec}(K * \varphi)$. Therefore, as $K * \varphi=\bigcap \operatorname{dec}(K * \varphi)$, we get that $K * \varphi=\bigcap \delta(\varphi)$. Thus, as $K *_{\delta} \varphi=\bigcap \delta(\varphi)$, we get $K *_{\delta} \varphi=K * \varphi$.

The representation result of the ERF class with the basic AGM revision postulates comprehends Theorems 3.10 and Theorem 3.11.

\subsubsection{Constellar Revision Function: Full AGM Revision Rationality}

Although the ECFs satisfy the basic AGM postulates, they are not strong enough to capture the supplementary postulates. In this section, we define a class of revision functions that satisfies the AGM revision supplementary postulates. The main idea is to constrain the class of ERFs in way that the choice function of each ERF picks its consistent complete theories according to a binary relation. We will impose two constraints on it: Mirroring, which was introduced in Section 3.3, and

( $\omega$-Maximal cut) for every consistent formula $\varphi \in L, \omega(\varphi)$ has a maximal element w.r.t $<$. 
At this point, we need to stress the differences and similarities between the $\bar{\omega}$-Maximal cut property introduced in Section 3.3 and the $\omega$-Maximal cut above. It turns out that both forms of maximal cut have more similarity than differences. Intuitively, both properties enforce a binary relation $<$ to ensure the existence of maximal elements in some desired subsets of complete consistent theories (either $\omega(\varphi)$ or $\bar{\omega}(\varphi)$ ). The interesting part is that in logics closed under classical negation, both properties mean the same thing.

Observation 3.8. In logics closed under classical negation, $\omega$-Maximal cut and $\bar{\omega}$-maximal cut are equivalent.

Proof Sketch. Under classical negation we have that $\bar{\omega}(\varphi)=\omega(\neg \varphi)$. Therefore, $\bar{\omega}$-maximal cut could be rewritten as: for every non-tautological formula $\varphi, \omega(\neg \varphi)$ has a maximal element w.r.t $<$. However, as $\neg \neg \varphi$ is a well-formed formula and therefore not inconsistent, since $\neg \varphi$ is not a tautology, we can rewrite $\bar{\omega}$-maximal cut once more as: for every consistent formula $\varphi, \omega(\varphi)$ has a maximal element w.r.t $<$. Note that this is actually $\omega$-Maximal Cut. Showing the converse direction (i.e. from $\omega$-Maximal cut to $\bar{\omega}$-maximal cut) is analogous.

In this section, unless otherwise specified, we will refer to $\omega$-maximal cut as simply maximalcut, and a binary relation that satisfies maximal-cut will be called a sovereign. Moreover, a casting function whose relation is a sovereign will be called a coalition. It is worth highlighting that though sovereign and contra-headed are equivalent definitions (as we are dealing with logics closed under classical negation), we use these two different terms to stress their difference in nature: contraheaded relations work over complements, whereas in sovereign relations the focus is on the opposite direction. In this section, we will focus only on sovereigns.

Definition 3.9. Let $<$ be a sovereign over $T_{L}$. A coalition is a function $\nu_{<}: L \rightarrow 2^{T_{L}}$ such that

$$
\nu_{<}(\varphi)= \begin{cases}\max _{<}(\omega(\varphi)) & \text { if } \operatorname{Cn}(\varphi) \neq C n(\perp) \\ \text { some } \emptyset \neq X \subseteq T_{L} & \text { otherwise }\end{cases}
$$

It is easy to notice that a coalition function is indeed a casting function.

Observation 3.9. Every coalition is a casting function.

It turns out that a coalition is all we need to capture both $\left(\mathrm{K}_{7}^{*}\right)$ and $\left(\mathrm{K}_{8}^{*}\right)$. A revision function constructed over a coalition function will be called a Constellar Revision Function.

Definition 3.10. Let $\nu_{<}$be a coalition w.r.t a sovereign $<$. A Constellar Revision Function (CRF) $*_{<}$is constructed from $<$as follows:

$$
K *<\varphi=\left\{\begin{array}{cl}
K+\varphi & \text { if } K+\varphi \neq L \text { or } C n(\varphi)=L \\
\bigcap \nu_{<}(\varphi) & \text { otherwise }
\end{array}\right.
$$

As a coalition function is a casting function, one can easily notice from the definition above that Constellar Revision Functions are ERFs, this means that every Constellar Revision Function satisfies the basic AGM revision postulates.

Observation 3.10. Every Constellar Revision Function is an Exhaustive Revision Function. 


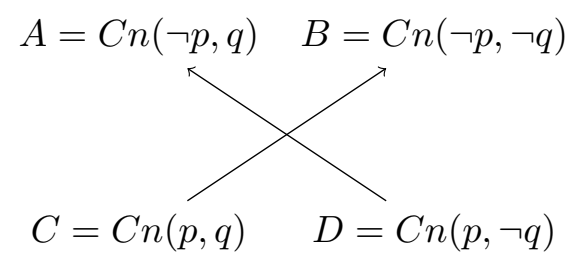

Figure 3.6: A sovereign relation for Example 10.

Proof. It is straightforward from the definition of Constellar function and ERFs.

Example 10 illustrates a Constellar revision function.

Example 10. Let $K=C n(\neg q)$ be a propositional theory, $<$ be the binary relation depicted in Figure 3.6, and $\nu_{<}$be the respective coalition function. The collection of consistent complete theories of the formulae $\neg p \vee q, p \vee q$ and $q$ are respectively $\omega(\neg p \vee q)=\{A, B, C\}, \omega(p \vee q)=\{A, C, D\}$, and $\omega(q)=\{A, C\}$. According to $<$ which is a sovereign: $\nu_{<}(\neg p \vee q)=\{A, B\}, \nu_{<}(p \vee q)=\{A, C\}$ and $\nu_{<}(q)=\{A, C\}$. Therefore,

$$
\begin{array}{lll}
K *<\neg p \vee q & =\bigcap \nu_{<}(\neg p \vee q) & =C n(\neg p) \\
K *<p \vee q & =\bigcap \nu_{<}(p \vee q) & =C n(q) \\
K *<q & =\bigcap \nu_{<}(q) & =C n(q) .
\end{array}
$$

Before we head to prove that every CRF satisfies the supplementary postulates, we will need first some properties regarding a theory and its complete consistent theories. We know that every consistent theory $K$ can be decomposed in terms of consistent complete theories, and that $\operatorname{dec}(K)$ is one of these decompositions. As a result, the theory $K+\varphi$, as long as it is consistent can also be decomposed in terms of consistent complete theories. Though we know that one of its decomposition is $\operatorname{dec}(K+\varphi)$, this does not give any construction rule or hint of how to reach $\operatorname{dec}(K+\varphi)$ departing from $\operatorname{dec}(K)$. This is a crucial property we will need in order to prove that Constellar Revision Functions satisfy $\left(\mathrm{K}_{7}^{*}\right)$. The proposition below answers exactly this question. It shows that the decomposition of $K+\varphi$ corresponds exactly to taking $\operatorname{dec}(K)$ and then intersecting it with the $\omega(\varphi)$ : the class of all consistent complete theories that entail $\varphi$. In a simpler way, $\operatorname{dec}(K+\varphi)=\operatorname{dec}(K) \cap \omega(\varphi)$. This property actually does not depend on the function dec and can be extended to any decomposition of $K$. Precisely, if $A$ is a decomposition of $K$, then $A \cap \omega(\varphi)$ is a decomposition of $K+\varphi$.

Proposition 3.11. Let $K$ be a theory, $\psi$ a formula and $A$ a set of complete theories such that $K=\bigcap A$. If $K+\psi$ is consistent, then $K+\psi=\bigcap(A \cap \omega(\psi))$.

Our first step towards our representation result comprehends the Theorem 3.12 and 3.13 below. Due to the sovereigns, a CRF is strong enough to capture postulate $\left(\mathrm{K}_{7}^{*}\right)$, however in order to capture $\left(\mathrm{K}_{8}^{*}\right)$ we need to enforce one more constraint upon a sovereign: mirroring. We will say that a CRF whose sovereign satisfies mirroring is a mirrored CRF.

Theorem 3.12. Every CRF satisfies $\left(K_{7}^{*}\right)$.

Theorem 3.13. Every mirrored CRF satisfies ( $\left.K_{8}^{*}\right)$. 
Now we will show that every fully rational AGM revision function is equivalent to some Constellar Revision Function. As every Constellar Revision Function satisfies the six basic AGM revision postulates, we know they are ERFs. Therefore, the only thing left to prove is that the casting function of a fully rational AGM revision function is indeed a coalition. In other words, given a casting function $\delta$ from an ERF that satisfies the supplementary postulates, the first step is to show that there is a sovereign $<\operatorname{such}$ that $\delta$ is equivalent to the coalition $\nu_{<}$. The first and most intuitive option is to construct for each formula $\varphi$, a relation $<_{\varphi}$ such that the maximal elements of $\omega(\varphi)$ ordered via $<_{\varphi}$ coincide with $\delta(\varphi)$, that is, $\delta(\varphi)=\max _{<\varphi}(\omega(\varphi))$. This can be done making

$$
<_{\varphi}=\{(X, Y) \in \omega(\varphi) \times \omega(\varphi) \mid Y \in \delta(\varphi) \text { and } X \notin \delta(\varphi)\}
$$

It is worth noting that when $\varphi$ is inconsistent, $<_{\varphi}=\emptyset$, as $\omega(\perp)=\emptyset$. Basically, $<_{\varphi}$ binds every element of $\omega(\varphi)$ not chosen by $\delta(\varphi)$ with every element of $\delta(\varphi)$. For instance, let us suppose that the set of all complete consistent theories for a given formula $\varphi$ is $\omega(\varphi)=\{A, B, C, D\}$. Moreover, let us take a casting function $\delta$ that picks $\{A, B\}$ for $\varphi$. The not chosen elements are $C$ and $D$, whereas the chosen ones are $A$ and $B$, therefore both $A$ and $B$ are preferable over $C$ and $D$. Thus, $<_{\varphi}=\{(C, A),(C, B),(D, A),(D, B)\}$ which is illustrated in Figure 3.7.

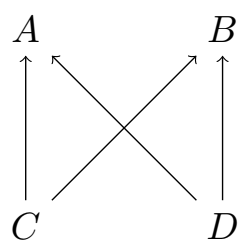

Figure 3.7: Depiction of a binary relation

This simply defines a relation upon $\omega(\varphi)$ whose maximal elements are the complete consistent theories of $\delta(\varphi)$. The tricky part is to construct the general relation, and simply putting every $<_{\varphi}$ together as a relation $<=\bigcup_{\varphi \in L}<_{\varphi}$ will not suffice. To achieve this, we will resort to the same trick we did for Blade Contraction Functions: resort to a kind of triangulation. We recall that a triangulation $\nabla(\varphi, \psi)$ relates non-maximal elements of a relation $<_{\varphi}$ to the maximal ones of a relation $<_{\psi}$. The only difference here is that in a blade contraction function, the relation $<_{\varphi}$ concerns elements of $\bar{\omega}(\varphi)$, whereas here for Constellar Revision Functions, a relation $<_{\varphi}$ concerns elements of $\omega(\varphi)$. Therefore, we will need to slightly change the definition of triangulation as follows:

Definition 3.11. Given a casting function $\delta$, and two formulae $\varphi$ and $\psi$. The positive-triangulation of $\varphi$ and $\psi$ w.r.t $\delta$ is the relation

$$
\boldsymbol{\nabla}(\varphi, \psi)=\{(A, B) \in \omega(\varphi) \times \omega(\psi) \mid A \in \omega(\varphi) \backslash(\delta(\varphi) \cup \delta(\psi)) \text { and } B \in \delta(\psi)\}
$$

The intuition behind the positive-triangulation is the same as in a triangulation, with the only difference that the latter looks for the complements of both $\varphi$ and $\psi$, i.e. $\bar{\omega}(\varphi)$ and $\bar{\omega}(\psi)$, whereas positive triangulation looks for complete consistent theories that entail either $\varphi$ or $\psi$, i.e. $\omega(\varphi)$ and $\omega(\psi)$. Employing positive-triangulation we can construct a relation which we will call the projection of $\delta$. A projection will be used to show that every fully rational AGM revision function is a Constellar Revision Function. 
Definition 3.12. Let $\delta$ be a casting function. Then the projection of $\delta$ is a relation $<_{\delta} \subseteq T_{L} \times T_{L}$ such that $(A, B)$ is in $<$ iff, for some formulas $\varphi$ and $\psi$ :

$$
\begin{aligned}
& \text { either }(A, B) \in\left(\bigcup_{\varphi \in L}<_{\varphi}\right) \\
& \text { or }(A, B) \in \mathbf{\nabla}(\varphi, \psi) \cup \mathbf{\nabla}(\psi, \varphi) .
\end{aligned}
$$

The role of a projection is to show that every fully AGM revision function $*_{\delta}$ is indeed a Constellar Revision Function. For this, we need to show such that the casting function $\delta$ from $*_{\delta}$ is actually a coalition, that is, there is a relation $<_{\delta} \operatorname{such}$ that $\delta(\varphi)=\max _{<\delta}(\varphi)$. To archive this, we can simply show that $<_{\delta}$ is actually the projection of $\delta$. The tricky part will be to prove that the projection is a sovereign, that is, it satisfies mirroring. To show this, we will need the properties $\mathbf{R} \mathbf{1}$ and $\mathbf{R 2}$ from Lemma 3.8, and restrict ourselves to saturated casting functions. A saturated casting function is a casting function $\delta$ that satisfies the saturation property:

Saturation: $\delta(\varphi)=\operatorname{dec}(\bigcap \delta(\varphi))$, if $\varphi$ is not a tautology.

Saturation was introduced in the Section 3.3 for saturated choice function. As for the BCF, restricting to saturated casting function do not exclude ERFs, that is, every casting function can be swapped by a saturated casting function and yet they will yield the same ERF, as pointed on Observation 3.11 below. For now on, unless otherwise specified, every casting function will be assumed to be saturated.

Observation 3.11. For every casting function $\delta$, there is a saturated casting function $\delta^{\prime}$ such that $K *_{\delta} \varphi=K *_{\delta^{\prime}} \varphi$.

Lemma 3.8. Let $*_{\delta}$ be an ERF, $K$ a theory and $\varphi$ and $\psi$ two consistent formulae such that $K *_{\delta} \varphi=\bigcap \delta(\varphi)$ and $K *_{\delta} \varphi \wedge \psi=\bigcap \delta(\varphi \wedge \psi)$. If $\delta$ is saturated then

(R1) if $*_{\delta}$ satisfies $\left(\boldsymbol{K}_{7}^{*}\right)$ then $\delta(\varphi) \cap \omega(\psi) \subseteq \delta(\varphi \wedge \psi)$; and

(R2) if $*_{\delta}$ satisfies $\left(\boldsymbol{K}_{8}^{*}\right)$ and $\delta(\varphi) \cap \omega(\psi) \neq \emptyset$ then $\delta(\varphi \wedge \psi) \subseteq \delta(\varphi) \cap \omega(\psi)$.

The conditions $\mathbf{R} 1$ and $\mathbf{R 2}$ follow from the postulates $\left(\mathrm{K}_{7}^{*}\right)$ and $\left(\mathrm{K}_{8}^{*}\right)$. The intuition behind $\mathbf{R} 1$ is that if a complete theory $S$ entails a formula $\psi$ and $S$ is chosen for the revision of a theory $K$ by a formula $\varphi$, then ( $\mathbf{K}_{7}^{*}$ ) compels $S$ to be chosen on the revision of $K$ by $\varphi \wedge \psi$. Regarding $\mathbf{R} \mathbf{2}$, for logics with negation if $\neg \psi$ is not in $K * \varphi \wedge \psi$ (in terms of consistent complete theories this reads as $\delta(\varphi \wedge \psi) \cap \omega(\psi) \neq \emptyset)$, then every complete consistent theory chosen for the revision of $K$ by $\varphi \wedge \psi$ must also be chosen to revise $K$ by $\varphi$.

Now we are ready to prove that every fully rational AGM revision function is a Constellar Revision Function. Since every rational AGM revision function is an ERF, our first step is to show that when an ERF satisfies the supplementary postulates, its casting function $\delta$ is a coalition. To show this, we take the projection $<_{\delta}$ of $\delta$ and we show that, except for the inconsistent formulae, the coalition $\nu_{<}$matches $\delta$. This is shown on the Proposition 3.12 below. The reason to let inconsistent formulae out of the game is because casting functions are not consulted in the presence of these formulae. We only need to enforce that for consistent formulae, a casting function of a fully AGM 
rational function chooses its consistent complete theories according to some sovereign (which is in this case its projection).

Proposition 3.12. Let $*_{\delta}$ be an Exhaustive Revision Function that satisfies $\left(K_{7}^{*}\right)$ and $\left(K_{8}^{*}\right), \delta$ be saturated, and $<_{\delta}$ the projection of $\delta$. For every consistent formula $\varphi, \delta(\varphi)=\nu_{<\delta}(\varphi)$.

As every casting function of a fully AGM rational revision function is a coalition, we can easily prove that it is a CRF:

Theorem 3.14. Let $*_{\delta}$ be an ERF that satisfies both $\left(K_{7}^{*}\right)$ and $\left(K_{8}^{*}\right), \delta$ be saturated and let $<_{\delta}$ be the projection of $\delta$. The $C R F *_{<\delta}$ is equivalent to $*_{\delta}$.

So far we have shown that every fully AGM revision function change function is a CRF and its casting function is indeed a coalition. The only thing missing is to prove that the projection of such a function satisfies mirroring. For this, we will explore an interesting sub-class of casting functions given in the following definition.

Definition 3.13. Let $K$ be a theory. A casting function $\delta$ is $K$-royal iff for every formula $\varphi$, if $K+\varphi \neq C n(\perp)$ then $\delta(\varphi)=\operatorname{dec}(K+\varphi)$.

The main idea behind a $K$-royal casting function is that whenever a formula $\varphi$ is consistent with a theory $K$, and therefore the revision of $K$ by $\varphi$ becomes $K+\varphi$, the complete theories chosen by $\delta$ coincide with the decomposition of $K+\varphi$, that is, $\delta$ selects all complete theories that contain $K+\varphi$.

Example 11. Let $K=C n(q)$, be a propositional theory, $<$ be the binary relation depicted in Figure 3.8, and $\nu_{<}$be the respective coalition function. This coalition function is $K$-royal. To see this, notice that the only formulae that are consistent with $K$ are those logically equivalent to $q, q \vee p, q \vee \neg p, q \wedge p$ and $q \wedge \neg p$. Moreover, let $\varphi$ be one of these formulae. Therefore, $K+\varphi \neq C n(\perp)$. It is easy to check that $\mu_{<}(\varphi)=\operatorname{dec}(K+\varphi)$, which means that $\mu_{<}$is $K$-royal.

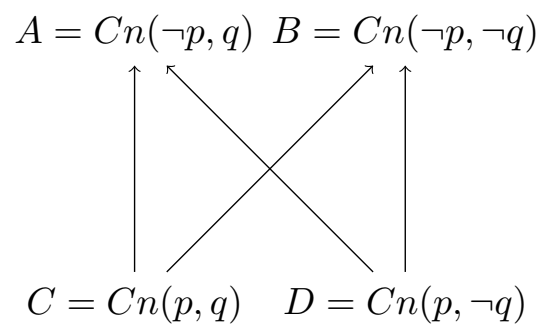

Figure 3.8: A binary relation < for the K-royal coalition function in Example 11.

The reason why we resort to $K$-royal casting functions is because they make it easier to show that their projections satisfy mirroring. The most important aspect of a $K$-royal casting function is the property given on the following corollary. Basically, it states that, as long as a formula is consistent, the result of the revision of $K$ by $\varphi$ corresponds to simply intersecting all the complete theories selected by $\delta(\varphi)$. The only exception is when $\varphi$ is inconsistent, which clearly corresponds to $C n(\perp)$ regardless of the theory $K$. 
Corollary 3.7. Given a theory $K$ and an $E R F *_{\delta}$. If $*_{\delta}$ is $K$-royal and saturated then for every consistent formula $\varphi, K *_{\delta} \varphi=\bigcap \delta(\varphi)$.

From the corollary above, it is easy to see that restricting to royal casting functions inflicts no damage. In other words, whatever casting function $\delta$ an ERF chooses to revise a theory $K$, there is a $K$-royal casting function $\delta^{\prime}$ such that a revision function constructed over either casting functions produces the same result:

Observation 3.12. Let $K$ be a theory, and $*_{\delta}$ an $E R F$, there is a saturated $K$-royal casting function $\delta^{\prime}$ such that $K *_{\delta} \varphi=K *_{\delta^{\prime}} \varphi$, for every formula $\varphi$.

As a coalition is a casting function, we can restrict ourselves to $K$-royal coalition functions in the sense that every coalition of a constellar revision function corresponds to a $K$-royal casting function. We will refer to these casting functions as $K$-royal coalition functions.

Theorem 3.15. Let $K$ be a theory, and $*_{\delta}$ an an ERF that satisfies both ( $\left.K_{7}^{*}\right)$ and ( $\left.K_{8}^{*}\right)$. If $\delta$ is saturated and $K$-royal then its projection $<_{\delta}$ satisfies mirroring.

Corollary 3.8. If an ERF $*_{\delta}$ satisfies both ( $\left.K_{7}^{*}\right)$ and $\left(K_{8}^{*}\right)$ and $\delta$ is saturated, then there is a CRF $*_{<}$equivalent to $*_{\delta}$ such that $<$ satisfies mirroring.

As every fully AGM rational revision function is an ERF, it is straightforward from Corollary 3.8 that every fully AGM rational revision function is equivalent to a CRF whose sovereign satisfies mirroring. Therefore, as from Theorems 3.12 and 3.13, mirrored CRF satisfy both $\left(\mathrm{K}_{7}^{*}\right)$ and $\left(\mathrm{K}_{8}^{*}\right)$, the so desired representation theorem between the eight AGM revision postulates and the mirrored CRF easily emerges:

Theorem 3.16. Every fully AGM rational revision function is a CRF $*_{<}$such that $<$satisfies mirroring.

\subsection{Levi Identity and Harper Identities}

An interesting aspect about contraction and revision functions is that they can be defined in terms of each other via the Levi and Harper identities:

$$
\begin{array}{ll}
\text { (Levi Identity) } & K * \varphi=(K \dot{-} \neg \varphi)+\varphi \\
\text { (Harper Identity) } & K \dot{-} \varphi=K \cap(K * \neg \varphi) .
\end{array}
$$

This interdefinability, however, is known to be established in the presence of the AGM assumptions which include compactness. Since we have shown that AGM-like contraction and revision functions can be defined in logics that are not compactness, we also investigate if this connection remains in logics without compactness. Indeed, Levi and Harper identities will only require a logic to be Tarskian and closed under classical negation and disjunction.

Theorem 3.17. If a contraction function $\dot{-}_{\delta}$ satisfies $\left(\boldsymbol{K}_{1}^{-}\right)$to $\left(\boldsymbol{K}_{4}^{-}\right)$and $\left(\boldsymbol{K}_{6}^{-}\right)$, then the revision operator $*$ obtained from $\dot{-}_{\delta}$ via Levi Identity satisfies the six AGM basic revision postulates. 
It is worth noticing that the recovery postulate $\left(\mathbf{K}_{5}^{-}\right)$plays no role to produce an AGM rational revision function via Levi identity. This connection also produces fully AGM rational revision functions, when the underlying contraction function satisfies the supplementary postulates. For this to happen, however, recovery cannot be dispensed. This is shown on Theorem 3.18, and the following observation will come in handy to prove that theorem.

Observation 3.13. If $\varphi \in K+\psi$ then $\psi \rightarrow \varphi \in K$, for every theory $K$.

Proof. As $\varphi \in K+\psi$, it follows that every model of $K$ that satisfies $\psi$ also satisfies $\varphi$. This means that $\psi \rightarrow \varphi \in K$.

Theorem 3.18. If a contraction function $\dot{-}_{\delta}$ is fully AGM rational, then the revision function * obtained via Levi identity is fully AGM rational.

While the Levi identity allows us to go from contraction function to revision function, the Harper identity allows us to go on the converse direction. As expected, the Harper identity produces AGM rational contraction functions from AGM rational revision functions. It is worth noticing that recovery is pulled back by Harper identity, unlike Levi identity which may push recovery out of the game.

Observation 3.14. If $\neg \psi \notin K+\varphi$, then $\varphi \rightarrow \neg \psi \notin K$.

Proof. Trivial: if we suppose that $\varphi \rightarrow \neg \psi \in K$, then $\neg \psi \in K+\varphi$ which is a contradiction.

Theorem 3.19. If a revision function $*$ satisfies $\left(\boldsymbol{K}_{1}^{*}\right)-\left(\boldsymbol{K}_{6}^{*}\right)$ then the contraction function $\dot{-}_{\delta}$ obtained via Harper identity satisfies $\left(\boldsymbol{K}_{1}^{-}\right)$to $\left(\boldsymbol{K}_{6}^{-}\right)$.

Similarly, if a contraction function is fully AGM rational, then the revision function obtained via Harper identity is also fully AGM rational. Observation 3.15 and Lemma 3.9 will be of immediate assistance to prove this in Theorem 3.20 below.

Observation 3.15 simply stress that if we remove $\varphi$ when retracting a conjunction $\varphi \wedge \psi$, then $\varphi$ will not return simply by adding the negation of this conjunction (i.e. $\neg \varphi \vee \neg \psi$ ) right after. Lemma 3.9 points that contracting a conjunction $\varphi \wedge \psi$ from a theory that does not posses $\varphi$ is equivalent, when using Harper Identity, to revise it by the negation of this conjunction (i.e. by $\neg \varphi \vee \neg \psi$ ).

Observation 3.15. If $\varphi \notin K \dot{-}(\varphi \wedge \psi)$ then $\varphi \notin K \dot{-}(\varphi \wedge \psi)+(\neg \varphi \vee \neg \psi)$.

Proof. Let us suppose for contradiction that $\varphi \in K \dot{-}(\varphi \wedge \psi)+(\neg \varphi \vee \neg \psi)$. Thus the formula $(\neg \varphi \vee \neg \psi) \rightarrow \varphi \in K \dot{-}(\varphi \wedge \psi)$. However $(\neg \varphi \vee \neg \psi) \rightarrow \varphi$ is equivalent to $\varphi \wedge \psi$. Thus, $\varphi \wedge \psi \in K \dot{-} \varphi \wedge \psi$ which is a contradiction.

Lemma 3.9. If $\varphi \notin K$, then $(K \cap K *(\neg \varphi \vee \neg \psi))+\neg \varphi \vee \neg \psi=K *(\neg \varphi \vee \neg \psi)$.

Theorem 3.20. If a revision function is fully AGM rational, then the contraction function obtained via Harper Identity is also fully AGM rational. 


\section{Chapter 4}

\section{KM Update and Non-Finitary Languages without Compactness}

The AGM paradigm, which we reviewed in Chapter 2, is undoubtedly the most influential theory of Belief Change. As we are about to see, this theory, however, is not strong enough to deal with all the aspects of belief change. To illustrate this, consider the following example.

Example 12. On Saturdays, John goes either to the ice rink ( $r$ ) or to the beach $(\neg r)$. John always go to the beach riding a bike (b), and to the ice rink by bus $(\neg b)$. His domestic robot, Troy, needs to prepare his lunch before he reaches back home. It knows that on Saturdays, John eats either fish and chips when coming from the beach, or pasta after figure skating. Troy receives a message from John informing the he has taken the bike due to a bus strike on that morning. Just after hanging up the phone, John steps into the ice rink and imagines that later when he reaches home Troy will be cooking his favourite pasta dish.

In the example above, Troy, the robot, believes that John is either on the ice rink ( $\mathrm{r}$ ) or on the beach. As John cannot be at two different places at the same time, we will represent the sentence "John is on the beach" by $\neg r$. Troy knows that John is on the ice rink if and only if John has not ridden his bike that morning. So, we can represent Troy epistemic state as $r \leftrightarrow \neg b$. When John informs Troy that he has taken the bike, Troy needs to change its beliefs. An AGM belief revision operation will make Troy conclude that John is on the beach. This, however, is not the case, as John is calling Troy from the ice rink.

This example shows that AGM belief revision does not deal accordingly with belief dynamics in all the circumstances. Winslett (1988) was the first to identify this issue in the context of reasoning about actions. She has then proposed a new change operation called the Possible Model Approach (PMA) to deal with this issue. Further, Katsuno and Mendelzon (2003) have extended the PMA to a more general class of belief change operations, and identified the rationality postulates that emerged from such constructions. This approach would be later on known as the KM paradigm due to the authors name.

The KM paradigm was initially designed for the classical propositional logics which makes its belief change constructions strongly dependent of propositional logics. In this work, we propose to take a step further, and we pose the following question: What happens to the standard KM update constructions when compactness is discarded? Interestingly, as in the case of AGM contraction 
Ribeiro et al. (2018), the correspondence between the standard KM constructions and the KM rationality postulates cease to exist. We identified that the reason for this is beyond compact. Precisely, the one to blame is the non-finatary property that usurps compactness in its absence:

non-finitary: a logic that has an infinity number of not logically equivalent formulae.

We call such logics (or languages) non-finitary since no assumption is being made as to if the alphabet in question is finite or not. For instance, the Propositional modal logic $\mathbf{K}$ is non-finitary and yet compact, where as temporal logics like LTL and CTL are neither finitary nor compact. Accordingly, instead of only dispensing with compactness, we shall also address the issue of belief update for non-finitary logics.

The first issue in bringing the KM paradigm to the realm of non-finitary logics concerns the representation of epistemic states. In the KM paradigm, an epistemic state is usually represented as a finite formula from propositional logic. The reason for this is only to give a finite representation of all the beliefs an agent has. When dealing with non-finitary logics, however, representing epistemic states in this way may be inconvenient. Mainly because in these logics not every belief set can be represented via a single formula or a finite set of formulae. For this reason, we shall translate here the KM paradigm in terms of epistemic states as theories. As in the AGM paradigm, an epistemic input is given as a formula $\varphi$, and there are three epistemic attitudes concerning an epistemic state $K$ and a formula $\varphi$ :

1. accept: if $\varphi \in K$;

2. reject: if $\neg \varphi \in K$, and $\varphi \notin K$;

3. ignore (or indeterminate): if neither $\varphi$ nor $\neg \varphi$ are in $K$.

The epistemic operations are

Update $(K \diamond \varphi)$ : adds $\varphi$ keeping the new epistemic state consistent, when both $K$ and $\varphi$ are consistent;

Erasure $(K \odot \varphi)$ : removes $\varphi$ from $K$, as long as $K$ is consistent.

We review in the next section the KM update operation. We translate the rationality postulates and the standard constructions of update considering epistemic states as theories. We will not cover the erasure operation in this work, which shall be left for future works.

The results of this chapter were partially published in (Ribeiro et al., 2019b)

\subsection{KM Update}

An update change concerns how an agent ought to change its beliefs in order to accommodate a new piece of information. This epistemic change is governed by a set of rationality postulates. We present these postulates translated in terms of epistemic states as theories: 
$(\mathrm{K} \diamond 1) \quad K \diamond \varphi$ is a theory;

$(\mathrm{K} \diamond 2) \quad \varphi \in K \diamond \varphi$;

$(\mathrm{K} \diamond 3) \quad$ If $\varphi \in K$, then $K \diamond \varphi=K$;

$(\mathrm{K} \diamond 4) \quad K \diamond \varphi$ is consistent, if both $K$ and $\varphi$ are consistent;

$(\mathrm{K} \diamond 5) \quad$ If $C n(\varphi)=C n(\psi)$, then $K \diamond \varphi=K \diamond \psi$;

$(\mathrm{K} \diamond 6) \quad K \diamond(\varphi \wedge \psi) \subseteq K \diamond \varphi+\psi ;$

$(\mathrm{K} \diamond 7) \quad$ If $\psi \in K \diamond \varphi$ and $\varphi \in K \diamond \psi$, then $K \diamond \varphi=K \diamond \psi$;

$(\mathrm{K} \diamond 8) \quad$ If $K$ is complete, then $K \diamond \varphi \vee \psi \subseteq(K \diamond \varphi) \cup(K \diamond \psi)$;

$(\mathrm{K} \diamond 9) \quad\left(K \cap K^{\prime}\right) \diamond \varphi=(K \diamond \varphi) \cap\left(K^{\prime} \diamond \varphi\right)$.

As we require an epistemic state to be a belief set, the postulate $(\mathrm{K} \diamond 1)$ is added here with the purpose to ensure that every update function goes from a belief set to another one.

Postulates $(\mathrm{K} \diamond 2)$ to $(\mathrm{K} \diamond 5)$ are inspired on the revision postulates $\left(\mathrm{K}_{2}^{*}\right)$ to $\left(\mathrm{K}_{6}^{*}\right)$. Postulate $(\mathrm{K} \diamond 2)$ is the success postulate, and postulate $(\mathrm{K} \diamond 5)$ is the postulate of extensionality, which dictates that update is not syntax sensitive. The other three postulates, however, deserves some explanation. Postulate $(\mathrm{K} \diamond 3)$ simply states that no change will be carried out, in the light of a formula $\varphi$ that is already believed. Postulate $(\mathrm{K} \diamond 4)$ is a weaker version of the consistency postulate of revision. It guarantees that the new epistemic state will be consistent, as long as the current epistemic state and the new formula are consistent. This is one of the main differences between revision and update. Katsuno and Mendelzon justify this weakening on consistency arguing that update should only reflect the changes that occurs in the environment. Therefore, if the epistemic state of an agent is inconsistent, this would be due to inconsistencies on the the description of the world, and such correction should be done via revision.

Postulate $(\mathrm{K} \diamond 6)$ corresponds to the supplementary postulate $\left(\mathrm{K}_{7}^{*}\right)$ of revision. Postulate $(\mathrm{K} \diamond 8)$ is a weak version of postulate $\left(\mathrm{K}_{8}^{*}\right)$ and concerns only complete theories. It states that whenever two formulae $\varphi$ and $\psi$ occur on the update of each other, updating by either $\varphi$ or $\psi$ should have the same effect. Finally, postulate $(\mathrm{K} \diamond 9)$ has the purpose to guarantee that each world will be considered independently.

The rationality postulates give rise to a class of belief change functions, here dubbed the Classical Update (CUP) functions ${ }^{1}$

$$
\text { CUP : } K \diamond \varphi=T h\left(\bigcup_{M \in[K]} \operatorname{Max}_{\leqslant_{M}}([\varphi])\right. \text {. }
$$

Given a theory $K$ and a formula $\varphi$, a CUP operator $\diamond$ behaves as follows. For each model $M$ of $K$, the operator $\diamond$ chooses from the models of $\varphi$ those ones closest to $M$. The notion of distance between models is usually given by a pre-order $\leqslant_{M}$. A model $M_{1}$ is closer to $M$ than a model $M_{2}$, if $M_{2} \leqslant{ }_{M} M_{1}$. These selected models are then assembled, and the theory of these models corresponds to $K \diamond \varphi$. Each model $M$ is assigned to a pre-order $\leqslant_{M}$ subject to the following constrain:

faithfulness: if $M^{\prime} \neq M$, then $M^{\prime}<_{M} M$, for every model $M^{\prime}$.

\footnotetext{
${ }^{1}$ Originally, a CUP function is defined over minimal elements, here for convenience we will resort to maximal elements, as we will need to deal with infinite chains, and maximal elements, in this case, make the reasoning task easier.
} 


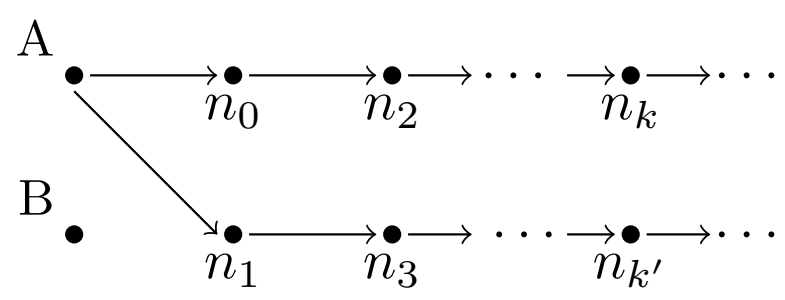

Figure 4.1: A preorder for the function in Example 13.

A function that assigns each model $M$ to a partial pre-order $<_{M}$ that satisfies the above constrains is called a faithful assignment. The intuition behind the faithfulness constrain is that a relation $<_{M}$ assigned to a model $M$ puts $M$ as the most preferable model of that relation.

Katsuno and Mendelzon (2003) show that in Propositional Logic, CUP functions and the KM update postulates are strongly connected.

Theorem 4.1. A belief change function satisfies the KM update postulates iff it is a CUP function.

\subsection{The Problem of Update in Non-finitary logics}

The connection between the KM update postulates and CUP functions cease for non-finitary logics. For instance, for non-finitary logics CUP functions do not satisfy postulate $(\mathrm{K} \diamond 7)$, as illustrated in Example 13.

Example 13. Let $\diamond$ be a CUP function over the pre-order $\leqslant$ depicted in Figure 4.1. An edge $x \rightarrow y$ means that $x \leqslant y$. Reflexive and transitive edges are omitted in the figure for convenience. Moreover, let $K$ be a complete consistent theory, and $\varphi$ and $\psi$ be two formulae such that $[\varphi]=$ $\left\{A, B, n_{0}, n_{2}, \ldots\right\},[\psi]=\left\{A, B, n_{1}, n_{3}, \ldots\right\}$, and $[\varphi \wedge \psi]=\{A, B\}$. According to the relation $\leqslant$, $\operatorname{Max}_{\leqslant}(\varphi)=\{B\}, \operatorname{Max}_{\leqslant}(\psi)=\{B\}$ and $\operatorname{Max}_{\leqslant}(\varphi \wedge \psi)=\{A, B\}$. Therefore,

$$
\begin{aligned}
K \diamond \varphi & =T h\left(\operatorname{Max}_{\leqslant}([\varphi])\right) & & =T h(\{B\}) \\
K \diamond \psi & =T h\left(\operatorname{Max}_{\leqslant}([\psi])\right) & & =T h(\{B\}) \\
K \diamond \varphi \wedge \psi & =T h\left(\operatorname{Max}_{\leqslant}([\varphi \wedge \psi])\right) & & =T h(\{A, B\}) .
\end{aligned}
$$

Though $\leqslant$ is a pre-order, the update function $\diamond$ does not satisfies (K॰7). The reason for this is that $(K \diamond 7)$ requires that $K \diamond \varphi=K \diamond \varphi \wedge \psi$, however this does not occur in this example. To see this, note that $\varphi \wedge \psi \in K \diamond \varphi$, as $B$ is model of both $\varphi$ and $\psi$. Clearly, $\varphi \in K \diamond \varphi \wedge \psi$. However,

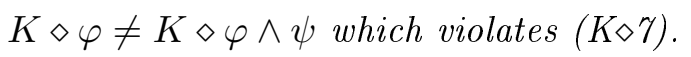

The reason why the function $\diamond$ of Example 13 does not satisfy $(\mathrm{K} \diamond 7)$ is related to the characteristic of non-finitary logics having an infinity number of models. In this case, a pre-order may induce an infinite chain in which it is not possible to determine a maximal (resp. minimal) element. To see how this issue is related to the infinity chains, let us see what happens to the $\diamond$ function of Example 13 if there were no infinity chain. For this, we can easily slightly change that example to consider a finite subset of elements: $n_{0}$ to $n_{3}$ besides $A$ and $B$. This gives us that $[\varphi]=\left\{A, B, n_{0}, n_{2}\right\}$ and $[\psi]=\left\{A, B, n_{1}, n_{3}\right\}$. Thus, $\left.\operatorname{Max} \leqslant(\varphi)=\left\{B, n_{2}\right\}\right)$ and $\left.\operatorname{Max}_{\leqslant}(\psi)=\left\{B, n_{3}\right\}\right)$. As $n_{2}$ is a model of $\varphi$ 


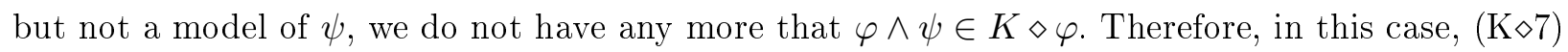
is trivially satisfied.

Example 13 shows us that the connection between KM update postulates and CUP functions breaks down in non-finitary logics. It is natural, then, to pose the following question: how can one perform belief update in non-finitary logics? Two natural answers are:

1. one can devise a new class of functions characterised by the KM update postulates in nonfinitary logics;

2. one can identify which set of postulates follow from CUP functions in non-finitary logics.

We pursue in this work both paths. We will propose a new class of functions that satisfy all the KM update postulates, and the results we obtain throughout this investigation will prove useful to show that CUP functions, in non-finitary logics, are characterised by a proper subset of the KM update postulates.

\subsection{Reconstructing Rational Update Functions}

In this section, we provide complete new accounts of rational KM update functions. For convenience, we split the KM postulates in two categories: basic postulates which comprises postulates $(\mathrm{K} \diamond 1)$ to $(\mathrm{K} \diamond 5)$ and $(\mathrm{K} \diamond 9)$; and supplementary postulates which consists of postulates $(\mathrm{K} \diamond 6)$ to $(\mathrm{K} \diamond 8)$. We will call a belief change function that satisfies the basic KM postulates a belief update function.

\subsubsection{KM Basic Rationality}

The functions we will devise here will operate over complete consistent theories rather than models, following our approach in Chapter 3. This will help up avoid technical issues. For instance, in modal logics, unlike in Propositional logic, two different models may satisfy exactly the same set of formulae.

Without compactness pre-orders are not strong enough to properly indicate the best elements to be used in order to update a theory $K$ by a formula $\varphi$, as illustrated in Example 13. This means we will need another way to define the 'closeness' criterion between consistent complete theories. We will assume that, instead of a pre-order, an agent has an appointee function that judges which complete theories of a formula $\varphi$ are closest to a complete theory $K$. This appointee function is used to perform the update of $K$ by $\varphi$. An appointee considers only consistent complete theories. We recall, from the Introductory chapter, Section 1.7, that $T_{L}$ stands for the set of all complete theories.

Definition 4.1. An appointee is a function $\delta: T_{L} \times L \rightarrow 2^{T_{L}}$ that maps each consistent complete theory and formula $\varphi$ to a set of consistent complete theories subject to:

(D1) $\delta(S, \varphi) \neq \emptyset$, if $S$ and $\varphi$ are consistent;

(D2) $\delta(S, \varphi) \subseteq \omega(\varphi)$ 
(D3) $\delta(S, \varphi)=\{K\}$, if $\varphi \in S$;

(D4) $\delta(S, \varphi)=\delta(K, \psi)$, if $C n(\varphi)=C n(\psi)$.

The purpose of an appointee is to select the best complete consistent theories that satisfy a formula $\varphi$ (condition D2). This criterion is given locally, and it depends on the fixed complete theory $S$ as background. An appointee is compelled to choose at least one consistent complete theory that contains $\varphi$, as long as $\varphi$ is consistent (condition D1). If a formula $\varphi$ belongs to a complete theory $S$, then there is no need to go after another theory, that is, the best theory for $\varphi$ is $S$ itself (condition D3). Finally, condition (D4) simply determines that an appointee is syntax independent.

The main purpose of defining an appointee function is that it captures the concept of distance between complete consistent theories in a general way. With an appointee in hand, it will become easier to explore which conditions we need to capture all the KM update postulates. We start by defining a new class of update functions, the splinter functions which is similar in spirit to CUP functions:

Definition 4.2. Given an appointee $\delta$, a splinter is a function $\diamond_{\delta}$ such that

1. if $K$ and $\varphi$ are consistent, then

$$
K \diamond_{\delta} \varphi=\bigcap_{S \in \omega(K)}(\bigcap \delta(S, \varphi))
$$

2. otherwise, $K \diamond_{\delta} \varphi=C n(\perp)$.

We recall from the introduction chapter that $\omega(K)$ corresponds to the set of all complete consistent theories that contains a theory $K$.

To understand how a splinter works, we will first need a property that follows from the converse of postulate $(K \diamond 4)$ which states that when $K \diamond \varphi$ is consistent both $K$ and $\psi$ are consistent. To see this, first notice that $\varphi$ cannot be inconsistent since is required to be in $K \diamond \varphi$. On the other hand, if $K$ were inconsistent, then we would have $\varphi \in K$, in which case traps $K \diamond \varphi$ in the inconsistent case. So the only options remaining is that both $K$ and $\varphi$ are consistent. Conversely, if either $K$ or $\varphi$ are inconsistent, we get that $K \diamond \varphi$ is inconsistent.

This case is captured by the second condition of splinter function definition. For the other cases, a theory $K$ is split into its decomposition of complete consistent theory. An appointee then is used to select for each of these consistent theories the best complete theories from $\varphi$. Thereafter they are all intersected and it results in $K \diamond \varphi$.

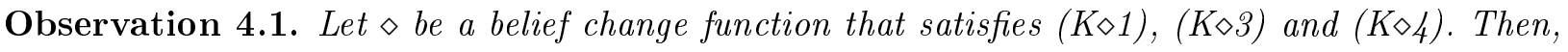
$K \diamond \varphi=C n(\perp)$ iff either $\varphi$ or $K$ is inconsistent.

Example 14. Let $K=C n(p)$ be a theory such that its decomposition has only two complete consistent theories: $K_{1}$ and $K_{2}$. Let an appointee $\delta$ be as follows: $\delta\left(K_{1}, \neg p\right)=\{C n(\neg p, q)\}$ and 
$\delta\left(K_{2}, \neg p\right)=\{C n(\neg p, \neg q)\}$. Thus,

$$
\begin{aligned}
K \diamond_{\delta} \neg p & =\delta\left(K_{1}, \neg p\right) \cap \delta\left(K_{2}, \neg p\right) \\
& =C n(\neg p, q) \cap C n(\neg p, \neg q) \\
& =C n(\neg p) .
\end{aligned}
$$

A splinter function behaves similarly to a CUP function, with the difference of resorting to an appointee instead of pre-orders to choose elements for the update. It is easy to check that every splinter function satisfies the basic KM postulates.

Theorem 4.2. Every splinter function $\diamond_{\delta}$ satisfies $(K \diamond 1)$ to $(K \diamond 5)$ and $(K \diamond 9)$.

Not surprisingly, splinter functions not only satisfy the basic KM update postulates, but every update function is a splinter function. To show this, first we will need some properties that follow from the basic KM postulates.

Another interesting property of update functions is that they are monotonic, that is, if $K \subseteq K^{\prime}$ then $K \diamond \varphi \subseteq K^{\prime} \diamond \varphi$. Update functions are monotonic due to postulate $(\mathrm{K} \diamond 9)$.

Proposition 4.1. Every belief change operation $\diamond$ that satisfies $(K \diamond 9)$ is monotonic.

The last piece of the puzzle towards our representation result consists in looking at splinter functions from another perspective. We begin with a special case: given that a theory $K$ is complete and $\varphi$ is a consistent formula, how does $K \diamond_{\delta} \varphi$ look like? As $K$ is a complete consistent theory, the only complete theory that contains $K$ is $K$ itself. Therefore, $\omega(K)=\{K\}$, which gives us a simpler version of splinter:

$$
K \diamond \varphi=\bigcap \delta(K, \varphi)
$$

Now let us proceed for a more general case. Imagine that $K$ is a consistent theory, but not complete. Let $S$ be one of the complete consistent theories from the decomposition of $K$, then have that $S \diamond \varphi=\bigcap \delta(S, \varphi)$. Thus, we can easily see that the update of $K$ by $\varphi$ consists in taking each $S \diamond \varphi$, for $S \in \omega(K)$, and intersecting all of them. This leads us to the following observation.

Observation 4.2. Given a splinter $\diamond_{\delta}$, a theory $K$ and a formula $\varphi$, if both $K$ and $\varphi$ are consistent then

$$
K \diamond_{\delta} \varphi=\bigcap_{S \in \omega(K)} S \diamond_{\delta} \varphi
$$

Observation 4.2 will be useful to prove the Theorem 4.3 below.

Theorem 4.3. Every update function is a splinter.

Our first representation result emerges from Theorems 4.2 and 4.3 which jointly show that splinter functions and the basic KM postulates are equivalent. 


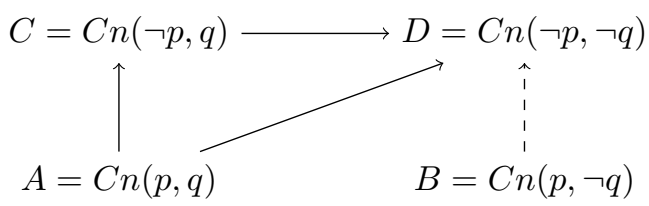

Figure 4.2: A Sovereign Relation.

\subsubsection{Revisiting the Supplementary Postulates}

Although splinter functions satisfy the basic KM postulates, they are not strong enough to capture the supplementary postulates. In this section we introduce a new class of update functions that satisfies the supplementary postulates. The main idea is to make the appointees to resort to binary relations in order to pick the consistent complete theories.

An appointee $\delta$ will assign, for each consistent complete theory $K$, a binary relation $\leqslant_{K}$. The appointee chooses the maximal elements within $\omega(\varphi)$ modulo $\leqslant_{K}$. These relations will need to satisfy some requirements in order to yield splinter functions that satisfies the supplementary postulates. The first one is

Maximal Cut: for every consistent formula $\varphi, \omega(\varphi)$ has a maximal element w.r.t $\leqslant$.

We recall that Maximal Cut was introduced in Chapter 3with the purpose to guarantee that for every formula $\varphi$, as long as it is consistent, the agent chooses at least one consistent complete theory of $\varphi$. This ensures that $\varphi$ will be successfully incorporated through the update process. We recall that a binary relation that satisfies maximal-cut is called a sovereign.

Appointee functions that rely strictly on sovereigns will be called relational appointees.

Definition 4.3. An appointee $\mu$ is relational iff for every consistent complete theory $K$, there is a sovereign $\leqslant_{K}$ such that $\mu(K, \varphi)=\max _{\leqslant K}(\omega(\varphi))$, for every consistent formula $\varphi$.

The new update functions we introduce are the royal splinter functions which consist of splinters functions founded on relational appointees.

Definition 4.4. A splinter $\diamond_{\mu}$ is royal iff its appointee $\mu$ is relational.

Example 15. Consider the complete theories depicted in Figure 4.2 and the respective sovereign $\leqslant$ represented by solid arrows. Let $\mu$ be a relational appointee such that for the theory $A$ it considers the sovereign $\leqslant$. Thus, $\mu(A, \neg q)=\{B, D\}$ which implies that

$$
A \diamond_{\mu} \neg q=B \cap D=C n(\neg q)
$$

At this point we investigate what we gain by restricting ourselves to royal splinters. The first benefit is that sovereigns guarantee satisfaction of postulates $(K \diamond 6)$ and $(K \diamond 8)$ which is shown on Theorem 4.4 below. Lemma 4.1 will be of immediate assistance thought this section.

Lemma 4.1. Let $\diamond_{\delta}$ be a royal splinter, and $K$ a complete consistent theory,

(i) if $\diamond_{\delta}$ satisfies $(K \diamond 6)$ then $\left.\delta(K, \varphi) \cap \omega(\psi) \subseteq \delta(K, \varphi \wedge \psi)\right)$;

(ii) if $\diamond_{\delta}$ satisfies $(K \diamond 8)$ then $\delta(K, \varphi) \cap \delta(K, \psi) \subseteq \delta(K, \varphi \vee \psi)$. 
The conditions (i) and (ii) from Lemma 4.1 concerns properties that follow from royal splinters, precisely the appointees of these functions, and are related respectively to postulates $(\mathrm{K} \diamond 6)$ and $(\mathrm{K} \diamond 8)$. Condition (i) points that if a consistent complete theory is considered on the update of a formula $\varphi$ and this same theory satisfies a formula $\psi$, then it is also selected for the update of the conjunction $\varphi \wedge \psi$. Similarly, condition (ii) states that if a consistent complete theory is taken on the update of both $\varphi$ and $\psi$, then it has to be taken on the update of $\varphi \vee \psi$.

Theorem 4.4. Every royal splinter satisfies $(K \diamond 6)$ and $(K \diamond 8)$.

\section{Strengthening Postulate $(\mathrm{K} \diamond 8)$}

At this point we need to discuss the role of postulate $(\mathrm{K} \diamond 8)$. Postulate $(\mathrm{K} \diamond 8)$ basically states that update of an epistemic state by a disjunction $\varphi \vee \psi$ is limited by the union of the update of its terms, that is, it is limited by the update of $\varphi$ assembled with the update of $\psi$. A more general way of seeing this postulate is that the update of a chain of disjunctions $\varphi_{1} \vee \cdots \vee \varphi_{k}$ is limited by the union of updating each of its terms $\varphi_{i}$, that is,

$$
K \diamond\left(\varphi_{1} \vee \cdots \vee \varphi_{k}\right) \subseteq K \diamond \varphi_{1} \cup \cdots \cup K \diamond \varphi_{k}
$$

In a short way, we can rewrite it as follows

$$
K \diamond(\bigvee \Gamma) \subseteq \bigcup_{\varphi \in \Gamma} K \diamond \varphi, \text { for a set of formulae } \Gamma
$$

The last form presents us with an interesting question: should we restrict $\Gamma$ to be a finite set of formulae? The concern for this is because some logics do not admit infinite formulae, therefore we could not always address to an infinite chain of disjunctions. Instead of constraining $\Gamma$ to be finite, we can swap the chain of disjunction for a even more general way. First, recall that a formula $\alpha$ is logically equivalent to a disjunction $\varphi \vee \psi$ if and only if the theory of $\alpha$ corresponds to the intersection of the theories of $\varphi$ and $\psi$. Precisely, $C n(\alpha)=C n(\varphi) \cap C n(\psi)$. Generalizing, we can then change the infinite disjunction by the intersection of the theories yielded by the formulae in $\Gamma$. This gives rise to the Disjunctive Coherence postulate (DisCo, for short).

DisCo: $K \diamond \psi \subseteq \bigcup_{\varphi \in \Gamma} K \diamond \varphi$, for each set of formulae $\Gamma$; if $C n(\psi)=\bigcap_{\varphi \in \Gamma} C n(\varphi)$, and $K$ is complete.

It is easy to note that for the case that $\Gamma$ is finite, both DisCo and $(\mathrm{K} \diamond 8)$ are equivalent. But, what happens then when $\Gamma$ is infinite? Does DisCo present any stronger behaviour than (K॰8) or not? To answer these questions, we will need to be a little bit more attentive to some technical details. First of all, we should ask what is the consequence of allowing $\Gamma$ to be infinite in propositional logic. Not surprisingly, it presents no difference since in propositional logic, as long as the number of propositional symbols is finite, the number of formulae that are not equivalent is finite. To illustrate this, note that for two propositional symbols, we can have at most four formulae that are not logically equivalent. Therefore, DisCo and $(\mathrm{K} \diamond 8)$ are equivalent in propositional logic, regardless 
of the restriction of $\Gamma$ being infinite or not. This changes, however, when we step off proposition logic to non-finitary logics, such as CTL and LTL, which even for a finite number of propositional symbols it presents an infinity number of distinct formulae. In this case, DisCo is indeed stronger than $(\mathrm{K} \diamond 8)$. To notice this, we illustrate in Example 16 an update function that although it satisfies $(\mathrm{K} \diamond 8)$ it does not capture DisCo.

Example 16. We construct an splinter $\diamond_{\delta}$ whose appointee $\delta$ is defined as follow. Let $\Gamma=\{p, X p$, $\left.X^{2} p, \ldots, X^{k} p, \ldots\right\}$, and $K$ a complete consistent theory. If $F p \in K$, then $\delta(K, \varphi)=\delta^{\prime}(K, \varphi)$. We will only need then to If $\varphi \in K$ then $\delta(K, \varphi)=\{K\}$. Otherwise,

1. $\delta(K, \perp)=T_{L}$;

2. $\delta(K, \varphi)=\omega(\varphi)$, if $G p \models \varphi$ and $\varphi \models(\bigvee S)$, for some some finite subset $S \subset \Gamma$;

3. $\delta(K, \varphi)=(\omega(\varphi) \backslash \omega(F p)) \cup \delta(\varphi \wedge F p)$, if $\varphi \not \models F p$ and $\varphi \wedge F p \not \neq \perp$;

4. $\delta(\varphi)=\omega(\varphi) \backslash \omega(G p)$, otherwise.

First we recall that in LTL $C n(F p)=\bigcap_{\varphi \in \Gamma} C n(\varphi)$. That is, the intersection of all formulae from $\Gamma$ yields the theory $C n(F p)$. It is important to highlight that $G p$ entails all formulae in $\Gamma$ and Fp itself. We split the intuition of how $\delta$ works into two main cases: when a formula $\varphi$ entails Fp and when it does not entail Fp.

Regarding a formula $\varphi$ that entails Fp, the appointee $\delta$ will behave either choosing all the theories from $\omega(\varphi)$ - case (2) - or taking $\omega(\varphi)$ and expelling the theories from $G p$ - case (4). If $\varphi$ is equivalent to a finite disjunction of the formulae in $\Gamma$, as for instance $p \vee X p$, then $\delta$ will keep all theories of $\varphi$; otherwise, it will exclude the theories from Gp. Note that the latter applies for Fp itself, as it is not equivalent to any finite disjunction of formulae in $\Gamma$. This is the reason why the splinter defined

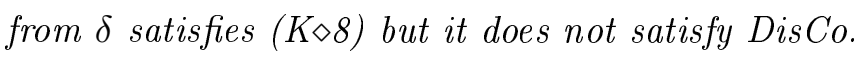

On the other cases, if $\varphi$ does not imply Fp, then we have two options: either its conjunction with Fp results in an inconsistent formula (which means that $\varphi$ and Fp shares no theory) or the conjunction of them is consistent. In the first case, we get the case (4). However, as Gp is not part of $\omega(\varphi)$, it turns out that we keep $\omega(\varphi)$ itself. In the latter case, we will keep the theories of $\omega(\varphi)$

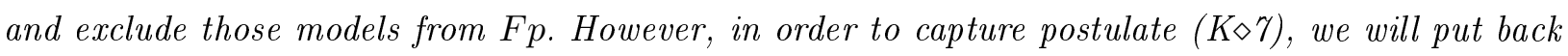
those theories selected from the conjunction $\varphi \wedge F p$ which clearly implies Fp. This case has a purely technical role to satisfy $(K \diamond 7)$. Since an appointee cannot choose an empty set, when formula is inconsistent we simply make $\delta$ picks all the consistent complete theory.

In order to show that the appointee from Example 16 yields a splinter that satisfies $(\mathrm{K} \diamond 8)$ but fails Disco, we will need the following Lemma of immediate assistance. It basically translates DisCo behaviour in terms of how an appointee behaves. Broadly speaking, it states that if an appointee $\delta$ chooses a theory $A$ for all formulae in a set $\Gamma$ then $A$ also should be picked for a formula $\alpha$ that is equivalent to the intersection of all theories of $\Gamma$.

Lemma 4.2. Given a set of formulae $\Gamma$ such that $\bigcup_{\varphi_{i} \in \Gamma} \omega\left(\varphi_{i}\right)=\omega(\psi)$. A splinter $\diamond_{\delta}$ satisfies DisCo iff $\bigcap_{\varphi_{i} \in \Gamma} \delta\left(\varphi_{i}\right) \subseteq \delta(\psi)$ 
Proof. It is straightforward from DisCo definition.

From this, we get that the update function $\diamond_{\delta}$ constructed from the appointee of Example 16 does not satisfy DisCo.

Observation 4.3. Let $\delta$ be the appointee from Example 16. The update function $\diamond_{\delta}$ does not satisfies DisCo.

Proof. It is straightforward from the definition of $\delta$. First notice that $\delta(K, F p)=\omega(F p) \backslash \omega(G p)$. To satisfy DisCo, it is necessary that $\bigcap_{\varphi \in \Gamma} \delta(K, \varphi) \subseteq \delta(K, F p)$. From the construction of $\delta$, we have that $\omega(G p) \in \delta(K, \varphi)$, for every $\varphi \in \Gamma$. This implies that $\omega(G p) \subseteq \bigcap_{\varphi \in \Gamma} \delta(K, \varphi)$. However, $\omega(G p) \nsubseteq \delta(K, F p)$. Therefore, $\bigcap_{\varphi \in \Gamma} \delta(K, \varphi) \nsubseteq \delta(K, F p)$ which means that $\diamond_{\delta}$ does not satisfy DisCo.

As for finite disjunction the appointee of Example 16 behaves as usual, it is easy to show that it satisfies $(\mathrm{K} \diamond 8)$. Not only this, but it also satisfies $(\mathrm{K} \diamond 6)$.

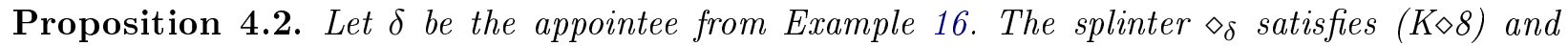
$(K \diamond 6)$.

As DisCo is stronger than $(\mathrm{K} \diamond 8)$, we argue that to deal with logics without compactness the most suitable is to swap $(\mathrm{K} \diamond 8)$ by DisCo. It is also important to highlight that even in the presence of $(\mathrm{K} \diamond 6)$, the postulate $(\mathrm{K} \diamond 8)$ does not capture $\mathrm{DisCo}$, as the update function from Example 16 satisfies $(\mathrm{K} \diamond 6)$. Also notice that every update function that satisfies DisCo also satisfies $(\mathrm{K} \diamond 8)$. For this reason, we will take DisCo as a suitable postulate to non-finitary logics rather than $(\mathrm{K} \diamond 8)$. Note, however, that this still ensures that $(\mathrm{K} \diamond 8)$ is satisfied.

Since we swap $(\mathrm{K} \diamond 8)$ for DisCo, the next task is to identify a class of splinters that capture DisCo. It turns out, that royal splinters are already strong enough for this.

Proposition 4.3. Every royal splinter satisfies DisCo.

From now own, we will say that an update function is fully KM rational if it satisfies the basic KM postulates, $(\mathrm{K} \diamond 6),(\mathrm{K} \diamond 7)$ and DisCo.

The next step is to capture the postulate $(\mathrm{K} \diamond 7)$. For this, we will need to impose one more restriction over the relations considered by a relational appointee:

Quasi-reflection: if $A \nless B$ and $B \not A$ but $A \leqslant C$ then

(i) either $B \leqslant C$, or

(ii) if $C \leqslant C^{\prime}$, then $B \leqslant C^{\prime}$.

To explain quasi-reflection, we will consider conditions (i) and (ii) separately, though very similar. The main intuition is that if an agent has no preference between two elements $A$ and $B$, then all elements preferable to $A$ should be preferable to $B$ and vice versa (i). The second condition elevates this mimicking process by one step. For instance, consider the relation $\leqslant$ depicted in Figure 4.2 by both solid and dashed arrows. In that relation, there is no preference between $A$ and $B$. Note that $A \leqslant C$ and $C \leqslant D$. However, $C$ is not preferable to $B$, as condition (i) demands. In this case, 
instead of forcing $B \leqslant C$, condition (ii) pushes $B$ to mimic one of the elements immediately above $A$, in this case mimic the preferences over $C$. Thus, it makes $B \leqslant D$ (depicted as a dashed arrow).

It turns out that quasi-reflection is all we need to capture $(\mathrm{K} \diamond 7)$. A relational appointee that considers only sovereigns that satisfy quasi-refection will be called a quasi-reflected appointee.

To see how quasi-reflection captures $(\mathrm{K} \diamond 7)$, we begin by looking at a special case of $(\mathrm{K} \diamond 7)$ over complete consistent theories.

Lemma 4.3. Let $\diamond_{\mu}$ be a royal splinter, such that $\mu$ is quasi-reflected. Given a complete consistent theory $K$, if $\varphi \in K \diamond_{\mu} \psi$ and $\psi \in K \diamond_{\mu} \varphi$, then $K \diamond_{\mu} \varphi=K \diamond_{\mu} \psi$.

The tricky part is to show that quasi-reflection not only captures $(\mathrm{K} \diamond 7)$ in complete theories, but also in every theory. We make use of the monotonicty property of update functions to show, from lemma above, that $(\mathrm{K} \diamond 7)$ spreads from complete consistent theories throughout over all theories.

Theorem 4.5. Given a royal splinter $\diamond_{\mu}$, if $\mu$ is quasi-reflected then $\diamond_{\mu}$ satisfies $(K \diamond 7)$.

We get the following corollary from Theorems 4.4 and 4.5 .

Corollary 4.1. A royal splinter whose appointee is quasi-reflected is fully KM rational.

We have reached the first piece of our representation result in form of the Corollary 4.1. The next step is to prove that every fully KM update function is a royal splinter function whose appointee is quasi-reflected, in short, a royal reflected splinter.

We begin by showing that fully KM update functions are royal. From Theorem 4.2 we have that every KM update function is a splinter, which means that all of them have an appointee. All we need to show now is that appointees from fully KM splinters are relational.

This is not a trivial task. We will start with a special case and then extend it to a more general case. This special case regards consistent complete theories. Let $K$ be a complete consistent theory, and $\diamond_{\delta}$ a splinter fully KM rational. We want to identify a sovereign $\leqslant_{K}$, such that $\delta(K, \varphi)=$ $\operatorname{Max}_{\leqslant K}(\omega(\varphi))$, for every consistent formula $\varphi$. For now, we will say that $\leqslant_{K}$ is the silhouette of $\delta$ within $K$.

To achieve this, let us assign to each formula $\varphi$ a relation $\leqslant_{\varphi}$ such that the maximal elements of $\omega(\varphi)$ modulo $\leqslant_{\varphi}$ match $\delta(K, \varphi)$. This can be easily achieved by making $\leqslant_{\varphi} \subseteq \omega(\varphi) \times \omega(\varphi)$ such that a pair $(A, B)$ is in $\leqslant_{\varphi}$ iff $A \notin \delta(\varphi)$ and

(i) either $A$ or $B$ are not in $\delta(\psi)$ for all non-tautological and consistent formula $\psi$.

Then, we can assemble all $\leqslant_{\varphi}$ together to reach the general relation $\leqslant_{K}$.

The intuition behind the construction of $\leqslant_{\varphi}$ is as follows. First, we want that the maximal elements of $\omega(\varphi)$ modulo $\leqslant \varphi$ matches $\delta(K, \varphi)$. To achieve this, we can basically pair each $A$ that is not chosen by $\delta$ with a theory $B$ that is chosen by $\delta$. This justify the condition that a pair $(A, B) \in \leqslant_{\varphi}$ if $A \notin \delta(K, \varphi)$. The condition (i) is a little bit tricky to understand. The main idea is that we do not need to pair a theory $A$ that is not in $\delta(K, \varphi)$ with all those that are in $\delta(K, \varphi)$. We need then a safe way to discard some of these unnecessary pairs. A better way to address the issue is to look from another perspective: imagine that for some formula $\psi$, both $A$ and $B$ are in $\delta(K, \varphi)$. In this case, it does not seem plausible to add $(A, B)$ in $\leqslant \psi$, neither is a good idea to add to $\leqslant \varphi$ 
since later on we will assemble $\leqslant_{\varphi}$ with $\leqslant_{\psi}$. Therefore, if we should not add $(A, B)$ to $\leqslant_{\varphi}$ if both are in $\delta(K, \psi)$, for some consistent and non-tautological formula $\psi$. The negation of this condition, then, clearly gives us then a condition of when we can safely add $(A, B)$ which clearly corresponds to the condition (i).

After assigning for each formula $\varphi$ a relation $\leqslant_{\varphi}$ as defined, we assemble all of them together to form a relation $\leqslant_{K}$. Although this seems a good idea, the relation $\leqslant_{K}$ constructed in this way is not strong enough to satisfy quasi-reflection.

In order to capture quasi-reflection, we adopt a variation of the triangulation employed in Chapter 3 .

Definition 4.5. Given a complete consistent theory $K$, an appointee $\delta$ and two consistent formulae $\varphi$ and $\psi$, the $\delta$-triangulation of $\varphi$ and $\psi$ modulo $K$ is the relation $\nabla(\varphi, \psi)=\{(A, B) \in \omega(\varphi) \times \omega(\varphi) \mid$ $A \in \omega(\varphi) \backslash(\delta(\varphi) \cup \omega(\psi))$ and $B \in \delta(K, \psi)\}$.

Triangulation was introduced in Chapter 3 in order to construct relations that satisfy quasireflected relations constrained by condition (i) - dubbed mirroring. The $\delta$-triangulation we employ here is a weaker variation, though the intuition is quite similar. Given a theory $K$, and a appointee $\delta$, a triangulation connects the complete consistent theories of $\varphi$ that were not chosen by $\delta$ to the theories of $\psi$ that were chosen by $\delta(K, \psi)$. This makes all non-chosen elements of $\varphi$ immediately less preferable than all elements chosen by $\psi$. The main difference between the triangulation version we use here from the original one is that we are indifferent about the complete theories that entails both $\varphi$ and $\psi$. For instance, if a complete theory $A$ entails both $\varphi$ and $\psi$ then $A$ will not participate in the triangulation $\nabla(\varphi, \psi)$.

Now we can go back to our problem of finding the silhouette of a complete consistent theory $K$ within an appointee $\delta$. This can be achieved by combining triangulation and our first naive idea of assembling relations $\leqslant \varphi$.

Definition 4.6. Given a complete theory $K$ and an appointee $\delta$, the projection of $\delta$ modulo $K$ is the relation $\leqslant_{K}$ such that $(A, B) \in \leqslant_{K}$ iff for some formulae $\varphi$ and $\psi$

$$
\text { either }(A, B) \in \leqslant_{\varphi} \quad \text { or } \quad(A, B) \in \nabla(\varphi, \psi)
$$

A projection concerns only the relation for a single complete consistent theory. To construct a relational appointee, we take the collection of all the projections of the consistent complete theories modulo $\delta$. We say that this is the shadow of $\delta$.

It turns out that the projection of $\delta$ modulo $K$ is also the silhouette of $\delta$ within $K$, when the splinter function $\diamond_{\delta}$ is fully KM rational.

Proposition 4.4. Consider a splinter $\diamond_{\delta}$, and a consistent complete theory $K$. If $\diamond_{\delta}$ is fully $K M$ rational, then the projection of $\delta$ modulo $K$ is the silhouette of $\delta$ within $K$.

An immediate consequence of this is that appointees of fully KM rational splinters are relational. To see this, notice that from the proposition above $\delta(K, \varphi)=\max _{\leqslant}(\omega(\varphi))$, for each consistent complete theory $K$, when $\diamond_{\delta}$ is fully KM rational.

Theorem 4.6. The appointee of a fully KM rational function $\diamond_{\delta}$ is relational. 
Hence we get that fully KM rational splinters are indeed royal.

Corollary 4.2. Fully KM rational splinters are royal splinters.

To show that fully KM rational functions are royal reflected splinters, we need only to show that fully KM rational splinters are reflected. For this, we need to show that the appointee of fully rational splinters are quasi-reflected. The triangulation used to construct projection gives us this property in an easy way.

Theorem 4.7. Let $\mu$ be the appointee from a fully KM rational splinter. Given a complete consistent theory $K$, the projection $\leqslant_{K}$ of $\delta$ modulo $K$ is quasi-reflected.

Therefore, we have that every fully KM rational belief change function is a royal reflected splinter. This together with Corollary 4.1 constitute the representation result between KM postulates and royal reflected splinters.

Corollary 4.3. An update function is fully KM rational iff it is a royal reflected splinter.

\subsection{Looking back at CUP functions}

At the previous section we also devised functions characterised by all the KM update postulates. In this section, we aim to investigate which class of postulates characterise the CUP functions in non-finitary logics. This question can be answered via some of the results achieved on the previous section.

It is easy to see, from both definitions of CUP and splinters that CUP functions are a special case of splinter functions, in which appointees work over preoreders. However, relying alone in preorders do not guarantee the postulate $(\mathrm{K} \diamond 2)$ of success, since it does not have the maximal-cut condition to guarantee the existence of maximal elements to be picked. Therefore, we will assume a stronger version of the CUP functions: that their preorders are sovereigns. Let us call it $\mathrm{CUP}^{+}$functions. Thus,

We explore the role of transitivity in royal splinters. Precisely, since transitivity does not capture postulate $(\mathrm{K} \diamond 7)$, it is natural to expect that a weaker postulate would emerge to fulfil the role of transitivity over royal splinters. We start by analysing how transitive connects with postulate $(\mathrm{K} \diamond 7)$ when the realm of models of a logic is finite. Thereafter, we extend it to the realm of non-finitary logics, and we identify $(\mathrm{K} \diamond 7)$ behaviour. As a consequence, we are able to identify a new rationality postulate for transitive royal splinters.

We recall the Example 13 that illustrates a $\mathrm{CUP}^{+}$function that fails $(\mathrm{K} \diamond 7)$. We argue that the reason for this is that transitivity captures this postulate when the number of complete consistent theories of a logic is finite. However, this connection cease when we depart to an infinite number of theories. If this is the case, then what is the role of transitivity regardless of the number of complete consistent theories of a logic being finite or not? To answer this, we need first to look at transitivity determines the maximal elements. Imagine that we have three elements $A, B$ and $C$ such that $A<B$ and $B<C$. Clearly, $A$ will never been chosen whenever it appears in a set with $B$. However, if we do not have $B$, it is possible that $A$ will be chosen, as for instance in the set $\{A, C\}$. Imposing transitivity, enforces that $A$ will never been chosen whenever $B$ is not preferable 
over another element, as for $\mathrm{C}$ for instance, even if $B$ is not present in that set. For instance, if we assume that $<$ is transitive, then even at $\{A, C\}, A$ loses its status of best element.

This makes us pose the following question: what happens then if the domain of selection never looks at a subset that contains both $A$ and $C$ ? To make it clearer, suppose that although the relation $<$ is constructed over $\{A, B, C\}^{2}$ a selection function that relies on $<$ is concerned only to the subsets $\{A, B\},\{B, C\}$ and $\{A, B, C\}$ (we will see that something very similar to this scenario happens in non-finitary logics, or in a number of them). In all these sets, transitivity adds no good for selection since whenever $A$ is in a subset, $B$ also is. This means that the transition $(A, C)$ does not remove $A$ from any of the selections. In this case, it makes no sense to enforce transitivity since $\mathrm{A}$ and $\mathrm{C}$ will never be put in consideration to compare preferences. This means that enforcing transitivity in these cases yields no interesting property for the selection criteria. Clearly, this does not happen in proposition logics since the preference order is given over the models of the logics and every subset corresponds to a formula. However, this phenomenon does not happens in every logic. Let us then look closely to how this affects the splinters.

Splinters are concerned to pick complete consistent theories that entail some formula $\varphi$. Therefore, if a set $S$ does not correspond to $\omega(\varphi)$ for some formula $\varphi$, then transitivity through $S$ will not lead any selection criteria. To see this, let $S=\{A, C\}$, and suppose that there is no formula $\varphi$ such that $S=\omega(\varphi)$. Thus, as we saw, transitive in this case will present nothing new. In order to formalize this phenomenon under which transitive is practically dispensed, let us look carefully at the set $S$. As it is a set of complete theories, we have that it induces a theory $K^{\prime}$, that is, the intersection of the theories in $S$ corresponds to $K^{\prime}$. However, such a theory cannot be generated via a single finite formula $\varphi$. We will call this property as $\alpha$-disruptive.

$\alpha$-disruptive: if there is a theory $K$ that entails $\alpha$ but it is not finite representable, that is, there is no formula $\psi$ such that $K=C n(\psi)$.

Our next step is to identify under which conditions transitivity will still be useful. Expanding the intuition of $(\mathrm{K} \diamond 7)$ in terms of infinity sets of theories can be understood as follows. Imagine a set of consistent complete theories $\Gamma$, such that the intersection of all these theories corresponds to the theory of a formula $\alpha$, that is,

$$
C n(\alpha)=\bigcap_{\psi \in \Gamma} C n(\psi)
$$

Moreover, let $\varphi$ be a formula such that the theories in $\delta(\varphi)$ appears in each $\delta(\psi)$, for $\psi \in \Gamma$. Assuming that we have no disruption of transitivity, we would get that $\delta(\varphi) \subseteq \delta(\alpha)$. It is not hard to see that in the case that the number of complete theories is finite this corresponds exactly to $(\mathrm{K} \diamond 7)$. This together with $\alpha$-disruption gives exactly the property we need to translate transitivity in terms of a general selection criteria:

T7 if $\delta(\varphi) \subseteq \omega(\alpha)$ and for all $\psi \in \Gamma, \delta(\varphi) \subseteq \omega(\psi)$ and $\delta(\psi) \subseteq \omega(\varphi)$ then either $\delta(\varphi) \subseteq \delta(\alpha)$ or there is some $S \subseteq \omega(\alpha)$ such that for all $\psi^{\prime}, \omega\left(\psi^{\prime}\right) \neq S$.

Translation of this property in terms of a rationality postulates gives us

$(\mathrm{K} \diamond 7 \mathrm{w})$ If $\alpha \in K \diamond \varphi$, and for all $\psi \in \Gamma, \varphi \in K \diamond \psi$ then either $K \diamond \alpha \subseteq K \diamond \varphi$ or there is some theory $K^{\prime}$ that is $\alpha$-disruptive, for all set of formulae $\Gamma$ such that $C n(\alpha)=\bigcap_{\psi \in \Gamma} C n(\psi)$. 


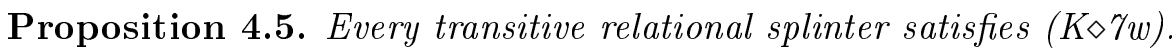

Therefore, transitivity looses its role in non-finitary logic which is seized by quasi-miroring. In this sense, the $\mathrm{CUP}^{+}$functions present as a too weak formalism to capture update functions.

Proposition 4.6. Every royal splinter function that satisfies $(K \diamond 6),(K \diamond 7 w)$ and $(K \diamond 8)$ is a $C U P^{+}$ function.

\subsection{Are total relations really needed?}

Besides the three KM supplementary postulates, the following postulate was proposed to be used in place of postulates $(\mathrm{K} \diamond 7)$ and $(\mathrm{K} \diamond 8)$ :

$(\mathrm{K} \diamond 10) \quad$ if $\mathrm{K}$ is complete and $\neg \psi \notin K \diamond \varphi$ then $K \diamond \varphi+\psi \subseteq K \diamond \varphi \wedge \psi$.

Postulate $(\mathrm{K} \diamond 10)$ is one of the AGM rationality postulate of belief revision. It actually actually corresponds to the postulate $\left(\mathrm{K}_{8}^{*}\right)$ of AGM revision. We rename it here as $(\mathrm{K} \diamond 10)$ only to make distinction of the study of this postulate in the context of Update rather then Revision. It is intuition was discussed in Chapter 3, Section 3.4. It basically dictates a rationality criterion that belief revision operators should follow in the light of conjunctive formulae.

In (Katsuno and Mendelzon, 2003), it is shown that the set of postulates resulted by swapping $(\mathrm{K} \diamond 7)$ and $(\mathrm{K} \diamond 8)$ for $(\mathrm{K} \diamond 10)$ characterises exactly the class of CUP functions defined over total preorders. Use of total pre-orders to represent preference relation of agent over its beliefs has received many criticisms (Lindström and Rabinowicz, 1991; Rott, 1992).

We show a new class of royal splinter functions characterised by the postulates $(\mathrm{K} \diamond 1)$ to $(\mathrm{K} \diamond 6)$ and $(\mathrm{K} \diamond 9)$ and $(\mathrm{K} \diamond 10)$. These functions, unlike in classical the approach, do not depend on total preorders.

As the basic KM postulates and $(\mathrm{K} \diamond 6)$ are satisfied by royal splinter functions, we will still maintain the restriction of appointees to be relational. To capture, $(\mathrm{K} \diamond 10)$, we will replace the quasi-reflection condition by the mirroring condition. Mirroring was introduced in Chapter 3 . We recall it here only for convenience,

(Mirroring) if $S_{1} \nless S_{2}$ and $S_{2} \nless S_{1}$; then for any $S^{\prime} \in T_{L}$, if $S_{1} \leqslant S^{\prime}$ then $S_{2} \leqslant S^{\prime}$.

It is worth highlighting that the mirroring corresponds to quasi-reflection constrained to its condition (i). Thus, every mirrored relation is a quasi-reflected relation.

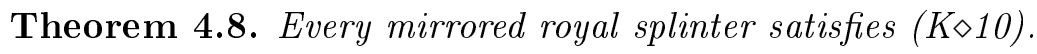

Now, let $\diamond_{\mu}$ be a splinter that satisfies $(\mathrm{K} \diamond 6)$ and $(\mathrm{K} \diamond 10)$. It can be shown that the projection $\leqslant_{K}$ of $\delta$ modulo $K$ is mirrored. This gives us that update functions that satisfies $(\mathrm{K} \diamond 6)$ and $(\mathrm{K} \diamond 10)$ are mirrored royal splinters.

Theorem 4.9. Every update function that satisfies $(K \diamond 6)$ and $(K \diamond 10)$ is a mirrored royal splinter. 


\subsection{Discussion}

In this work, we have addressed the issue of KM update in the context of non-fnitary logics. We have found both negative and positive results. On the negative side, the CUP functions, (fully) characterised by the KM update postulates in Propositional Logic, cannot satisfy all the postulates without compactness. We showed that without compactness CUP functions, and a slightly stronger version of them (the $\mathrm{CUP}^{+}$) are characterised by a proper subset of the KM rationality postulates. Assuming the KM update postulate as a good guide for belief update, we have devised a new class of update functions, the royal splinter functions, which are (fully) characterised by the KM postulates.

Another point we explored in this chapter was the consequences of swapping two of the KM supplementary postulate, precisely $(\mathrm{K} \diamond 7)$ and $(\mathrm{K} \diamond 8)$, by one of the AGM revision postulates: conjuctive preservation $(K \diamond 10)$. We have shown that, unlike in Propositional Logic, the set of postulates that emerges from replacing these postulates do not depend on total preorders. Instead, we replaced the total restriction by the mirroring condition, and obtained a complete characterisation between mirrored royal splinters and these another set of postulates. One advantage of mirroring is that it sets free the relations from the totality constraint which has received many criticisms.

We intend to explore, in the future, another forms of belief change in the context of non-finitary logics: the erasure functions. Our intention is to provide complete accounts of KM belief change for non-finitary logics. 


\section{Chapter 5}

\section{AGM Belief Revision and Non-Monotonic Systems}

Classical logics are monotonic: accumulation of new information (in form of additional premises) does not invalidate old conclusions. For instance, since from All birds fly and Tweety is a bird we can infer Tweety flies, acquiring another piece of information, Tweety is a penguin is not going to invalidate that inference - we will, classically, still be able to infer that Tweety flies. However, commonsense dictates that in light the new piece of information, that Tweety is a penguin, we should no longer be able to infer that Tweety flies since we "know" that penguins, though birds, cannot fly. Commonsense reasoning is non-monotonic: although some set of premises $\Delta$ entails some conclusion $x$, the inference of $x$ from $\Delta^{\prime}$ may not be guaranteed even if $\Delta \subseteq \Delta^{\prime}$. This realisation has led to the development of a number of approaches to non-monotonic logics, including, default logics (Besnard, 2013), defeasible logics (Nute, 1994) and expectation logics (Gärdenfors and Makinson, 1994).

One may attribute the non-monotonic behaviour of the commonsense inference to our tendency to "deactivate" some premises in light of some conflicting information - it is as if the new information, Tweety is a penguin, triggers deactivation of the premise, All birds fly. When viewed from this angle, non-monotonic reasoning appears to be closely connected to accounts of rational belief change, such as the classic AGM account, (Alchourrón et al., 1985), further developed in many works such as (Gärdenfors, 1988; Hansson, 1999; Rott, 1992). Indeed it has been argued that belief dynamics and non-monotonic reasoning are different perspectives on the same phenomenon (Makinson and Gärdenfors, 1991): I am licensed to (common-sensically/non-monotonically) infer sentence y from sentence $x$ if it is rational on my part to believe $y$ after accepting evidence $x$. This idea is concisely captured in the standard notation:

BRNM: $x \sim_{K} y$ if and only if $y \in K * x$

where $K$ represents a contextually fixed background knowledge, $\sim_{K}$ is the non-monotonic inference operation that employs $K$ in the background, and $*$ is a belief revision operation that yields the new "belief set" $K * x$ from the old belief set $K$ in light of evidential input $x$. Oftentimes the subscript ${ }_{K}$ from the relation $\sim_{K}$ is dropped for notational convenience when the intention is clear from the context. 
The connection between belief revision and non-monotonic reasoning captured by BRNM comes out handy in going back and forth between these two systems. Consider for instance a constraint on belief revision, that revision of a set of beliefs $K$ by evidence $x$ should not license one to believe in something that does not follow from $K$ and $x$ together, that is,

Inclusion: $K * x \subseteq C n(K \cup\{x\})$

where the consequence operation $C n$ represents the background (classical) logic. Assuming $C n$ satisfies Deduction, this constraint on belief revision can be rewritten as:

If $y \in K * x$, then $x \rightarrow y \in K$.

Now, since a tautology $\top$ brings in no new information, we can take $K * \top$ to be equivalent to $K$, which allows us to rewrite Inclusion as:

If $y \in K * x$, then $x \rightarrow y \in K * \top$.

Since this version of Inclusion is of the same syntactic form as BRNM, we get

Weak Conditionalisation: If $x \mid \sim y$, then $\top \sim x \rightarrow y$

which says that if $y$ is a non-monotonic consequence of $x$, then the material conditional $x \rightarrow y$ should be a theorem of that non-monotonic system. Principles of non-monotonic logic can similarly be translated to and viewed as principles of belief revision. The translation process is not always as straightforward as in this example; a rigorous recipe for this translation procedure as well as a comprehensive list of such translations can be found in the seminal work (Makinson and Gärdenfors, 1991). This work was later generalised to non-monotonic reasoning based on expectation orderings, ordering over sentences reflecting the extent they are expected to hold true (Gärdenfors and Makinson, 1994). As noted in (Dix and Makinson, 1992), non-monotonic reasoning based on expectation orderings is very similar to the classic approach to non-monotonic reasoning based on preference structures, often called the KLM-system, as propounded in (Kraus et al., 1990).

The belief revision operation $*$ alluded to above cooresponds to the AGM belief revision operator (see Chapter 2, for details).

In this thesis, we have been exploring the issue of belief revision on the context of non-compact logics. Since non-monotonic reasoning and belief revision are strongly connected, the nature of non-monotonic reasoning is worth enquiring when the background logic of the corresponding belief revision is not assumed to be compact. That is precisely the problem we address in this chapter. In particular, we study the connection between the AGM paradigm of belief revision and the nonmonotonic logic based on expectation orderings, and show that when compactness is dropped, it is still possible to walk from belief revision to non-monotonic reasoning. However, the walking back from non-monotonic reasoning to belief revision cannot be assured without further appropriate measures, and we identify such measures.

We review in the next section, Section 5.1, the AGM account of belief revision (Alchourrón et al., 1985) as well as the non-monotonic inference system based on Expectation orderings (Gärdenfors and Makinson, 1994), and indicate the significance of the compactness assumption. This is followed by a demonstration in Section 5.2 that relaxing the compactness assumption does not affect the 
transition from belief revision to non-monotonic reasoning. We show in the next section, Section 5.3 , and explain why, the trip back from non-monotonic systems to belief revision cannot be completed in absence of the compactness assumption. The subsequent Section 5.4 is devoted to identify the required conditions under which the desired connection between the belief revision and nonmonotonic reasoning can be established in the absence of compactness and provide the expected representation results. Finally, in section 5.5, we conclude this chapter with a brief discussion and considerations of future works. The results of this chapter were published at (Ribeiro et al., 2019a).

\subsection{Non-monotonic reasoning}

Unlike in the AGM approach to belief revision, in the non-monotonic reasoning system based on expectation orderings there are seven basic axioms and two supplementary axioms. This separation into two groups, first proposed in (Gärdenfors and Makinson, 1994), makes the alignment of these axioms with the postulates for AGM belief revision explicit.

We will assume that a non-monotonic inference relation, denoted $\sim$, builds up upon to an underlying logic $C n$. We will assume very little about $C n$, except that it is required to be Tarskian. The basic postulates (or axioms) for non-monotonic inference relation $\sim$ are:

$\begin{array}{lll}\text { N1 } & \text { If } \psi \in C n(\varphi) \text {, then } \varphi \sim \psi & \text { (Supraclassicality) } \\ \text { N2 } & \text { If } C n(\varphi)=C n(\psi) \text { and } \varphi \sim \alpha \text { then } \psi \sim \alpha & \text { (Left Logical Equivalence) } \\ \text { N3 } & \text { If } \varphi \sim \psi \text { and } \alpha \in C n(\psi) \text { then } \varphi \sim \alpha & \text { (Right Weakening) } \\ \text { N4 } & \text { If } \varphi \sim \psi \text { and } \varphi \sim \alpha \text { then } \varphi \sim \psi \wedge \alpha & \text { (And) } \\ \text { N5 } & \text { If } \varphi \sim \psi \text { then } \sim \varphi \rightarrow \psi & \text { (Weak Conditionalization) } \\ \text { N6 } & \text { If } \psi \neg \varphi \text { and } \sim \varphi \rightarrow \psi \text {, then } \varphi \sim \psi & \text { (Weak Rational Monotony) } \\ \text { N7 } & \text { If } \varphi \sim \perp \text { then } C n(\varphi)=C n(\perp) & \text { (Consistency Preservation). }\end{array}$

For simplicity, we will call any inference relation that satisfies the above seven (basic) postulates of non-monotonicity a non-monotonic inference relation.

Besides these basic postulates, two supplementary postulates were also introduced for nonmonotonic reasoning:
N8 If $\varphi \wedge \psi \sim \phi$ then $\varphi \sim \psi \rightarrow \phi$
(Conditionalization)
N9 If $\varphi \not \alpha \neg \psi$ and $\varphi \mid \sim \phi$ then $\varphi \wedge \psi \sim \phi$
(Rational Monotony).

Let us, for the time being, assume that $C n$ satisfies Compactness. Then it is easily verified that postulates N3 and N4 jointly imply the following closure axiom:

Closure $_{\mathrm{NM}}$ If $\varphi น \psi$ for all $\psi \in A$, then $\varphi \sim \alpha$ for all $\alpha \in C n(A)$.

named after the closure postulate $\left(\mathbf{K}_{1}^{*}\right)$ of belief revision that it corresponds to (Gärdenfors and Makinson, 1994). On the other hand, postulates N5 through N7 respectively correspond to the AGM revision postulates $\left(\mathbf{K}_{3}^{*}\right)$ through $\left(\mathbf{K}_{5}^{*}\right)$. Furthermore, postulate $\mathbf{N 2}$ corresponds to $\left(\mathbf{K}_{6}^{*}\right)$ (extensionality). Similarly, postulates N8 and N9 correspond respectively to the revision postulates 
$\left(\mathbf{K}_{7}^{*}\right)$ and $\left(\mathbf{K}_{8}^{*}\right)$ - see Chapter 2 for a complete list of the postulates and a discussion about their rationality criteria.

We will see in Section 5.3 that this nice correspondence between the revision operation $*$ and the inference relation $\sim$ breaks down at many points when the compactness assumption is dropped. Here we give an indication of how compactness can have consequences for non-monotonic reasoning systems. Let us consider Closure $_{\mathrm{NM}}$ which follows from axioms N3 and N4 when $C n$ satisfies compactness. However, in absence of compactness, Closure ${ }_{\mathrm{NM}}$ no longer follows from N3 and N4.

Theorem 5.1. If the underlying logic $C n$ is not compact, then $\mathrm{N} 3$ and $\mathrm{N}_{4}$ do not imply Closure $_{\mathrm{NM}}$.

Proof Sketch. Assume that $C n$ is not compact. Also suppose that $\sim$ is a nonmonotonic inference relation that satisfies $\mathbf{N} 3$ and $\mathbf{N} 4$. Let $\varphi$ be such that $\varphi \sim \beta$ for all $\beta \in A$ for some (infinite) set of formulae $A, \alpha \in C n(A)$, but $\alpha \notin C n\left(A^{\prime}\right)$ for any finite subset $A^{\prime}$ of $A$. Satisfaction of Closure $\mathbf{N M}_{\mathrm{NM}}$ would require that $\varphi \sim \alpha$. However, N3 and N4 would jointly allow non-monotonic derivation of $\alpha$ only if $\alpha \in C n\left(A^{\prime}\right)$ for some finite subset $A^{\prime}$ of $A$ contradicting our assumption.

\subsection{From * to $\sim$ without Compactness}

We recall from the introductory section the inter-translatability between revision operation * and non-monotonic inference relation $\sim$ captured by BRNM:

$\psi \in K * \varphi$ if and only if $\varphi \sim \psi$.

This allows us to navigate between a belief revision operator $*$ and an inference relation $\sim$. For convenience, when an inference relation $\mid \sim$ is taken to have been obtained from a revision operation $*$, we say that * induces $\sim$. Conversely, when navigating on the opposite direction, we will say that $\sim$ induces $*$. We will not assume that the background logic $C n$ is compact.

Let us at this point make the following observation, that if we start with a revision operator *, obtain the inference $\sim$ induced by it, and then again obtain the revision operator induced by this inference relation in turn, we will get back the revision operator $*$ we started with. Similarly, if we started with an inference operation $\sim$ and obtain another inference via a revision operation induced by it, we will go back to our original inference operation $\sim$. This simple observation will give us much flexibility with writing the proofs, and we will implicitly assume it throughout.

Observation 5.1. Let $*$ be a belief change operator and $\sim$ an inference relation. Further more, with a bit of notational overloading, let us denote by $\sim(*)$ the inference relation induced from *, and similarly, by $*(\sim)$ the belief revision operator induced by $\sim$. Then,

$$
\begin{aligned}
& \text { (a) } *(\sim(*))=* \text {, and } \\
& \text { (b) } \sim(*(\sim))=\sim \text {. }
\end{aligned}
$$

Proof. Let $K$ be fixed. We sketch the proof of (a) here, and the proof of (b) is analogous. We need to show that

$$
\alpha \in K * \varphi \text { iff } \alpha \in K *(\sim *) \varphi
$$

( $\Rightarrow$.) Suppose $\alpha \in K * \varphi$. Thus, $\varphi \sim(*) \alpha$, which implies that $\alpha \in K *(\mid \sim(*)) \varphi$. 
$(\Leftarrow$.) Suppose $\alpha \in K *(\sim(*))$. Then, $\varphi \sim(*) \alpha$ which implies that $\alpha \in K * \varphi$.

First we show that as long as $*$ satisfies the six basic AGM revision postulates, the induced $\sim$ relation satisfies the seven basic axioms of non-monotonicity (Theorem 5.2). Towards this end we first make the following useful observation which trivially follows from Deduction.

Observation 5.2. If $\varphi \in K+\psi$ then $\psi \rightarrow \varphi \in K$, for every theory $K$.

Theorem 5.2. If a belief change operator $*$ is (basic) AGM rational then its induced inference relation $\sim$ is a non-monotonic inference relation.

As the basic revision postulates translates to the basic non-monotonic axioms, the next step is to investigate if the supplementary postulates also imply the supplementary non-monotonic axioms. In Corollary 5.1 below we show that a fully AGM rational belief revision operator induces a non-monotonic inference relation that satisfies the supplementary axioms of non-monotonicity. Theorem 5.3 below shows a strong alignment, respectively between the revision postulates $\left(\mathbf{K}_{7}^{*}\right)$ and $\left(\mathbf{K}_{8}^{*}\right)$, and the axioms of non-monotonicity $\mathbf{N 8}$ and N9.

Theorem 5.3. Let $*$ be a belief revision operator that satisfies $\left(\boldsymbol{K}_{1}^{*}\right)$, and $\sim \sim$ be its induced inference relation. Then,

(i) if $*$ satisfies $\left(\boldsymbol{K}_{7}^{*}\right)$, then $\sim$ satisfies $\mathbf{N 8}$;

(ii) if $*$ satisfies $\left(\boldsymbol{K}_{8}^{*}\right)$, then $\sim$ satisfies $\mathbf{N g}$.

Putting Theorems 5.2 and 5.3 together tells us that a fully AGM rational belief revision operator induces a non-monotonic inference relation that satisfies the supplementary axioms of nonmonotonicity:

Corollary 5.1. Let $*$ be a fully AGM rational revision operation. The non-monotonic inference relation $\sim$ induced by it satisfies the two supplementary axioms of non-monotonicity.

What remains to be shown is that the supplementary axioms N8 and N9 induce belief change operators that satisfy respectively $\left(\mathbf{K}_{7}^{*}\right)$ and $\left(\mathbf{K}_{8}^{*}\right)$. However, we postpone this discussion to Section 5.4 , since the transition from the basic non-monotonic inference axioms to the basic AGM postulates proves problematic, as we show in the next section.

\subsection{From $\sim$ to $*$ without Compactness}

We have shown that a rational belief revision function behaves as a non-monotonic inference relation. Moreover, we also saw that the supplementary revision postulates imply the supplementary non-monotonic axioms, where: N8 is obtained from $\left(\mathbf{K}_{7}^{*}\right)$, and $\mathbf{N 9}$ from $\left(\mathbf{K}_{8}^{*}\right)$. In this section, we examine the converse problem, that is, if every non-monotonic inference induces a rational AGM revision operator.

As we will see, the basic non-monotonic axioms are too general to capture some of the basic AGM postulates in absence of compactness. We subsequently identify the conditions under which a non-monotonic inference relation behaves in accordance with the belief revision postulates. 
Let us first show that the basic non-monotonic axioms do not correspond to the basic AGM revision postulates. For this purpose we need to construct a non-monotonic inference relation $\downarrow$ such that the revision function $*$ induced by it violates some of the basic AGM postulates. We will conveniently take a revision function $*$ which violates one of the AGM postulates (namely, $\left(\mathbf{K}_{3}^{*}\right)$ ), and then we show that the non-monotonic inference relation $\sim$ induced by it satisfies all the basic non-monotonic axioms. In light of Observation 5.1, this will suffice our purpose. We construct such a belief revision operator in Example 17.

Example 17. Let $\otimes$ be a fully $A G M$ rational revision function, and $p$ an arbitrary formula. The belief revision operation $*$ is constructed as follows:

$$
K * \varphi= \begin{cases}(K+\varphi)+p & \text { if } \varphi \rightarrow \neg p \notin K \\ K \otimes \varphi & \text { otherwise. }\end{cases}
$$

The revision operation from Example 17 behaves in a simple way. It adds both $p$ and a formula $\varphi$ to $K$, if both $\varphi$ and $p$ are jointly consistent with $K$. On the other hand, if $K$ is inconsistent with $\varphi$ or $p$, it resorts to the rational AGM function $\otimes$. It is trivial to show that although it violates postulate $\left(\mathbf{K}_{3}^{*}\right)$, the inference relation $\sim$ induced by it satisfies $\mathbf{N} 1$ to $\mathbf{N} 7$. This example helps us to prove the following interesting result:

Theorem 5.4. Let $\sim$ be the inference relation induced from the belief change function $*$. It is not necessary for * to satisfy $\left(K_{1}^{*}\right)-\left(K_{6}^{*}\right)$ in order that $\sim$ satisfies all the basic axioms of nonmonotonicity.

Which trivially yields the following as a corollary:

Corollary 5.2. There is a non-monotonic inference relation $\sim$ whose induced belief change operator * violates some AGM revision postulates.

One might wonder that although the basic non-monotonic axioms do not lead to the six basic AGM postulates, perhaps the non-monotonic supplementary axioms can play a compensating role. However, the inference relation $\sim$ induced from the belief change function of Example 17 already satisfies these supplementary axioms. This means that even in the presence of the supplementary axioms it is not possible to navigate from a non-monotonic inference relation to the AGM postulates.

Observation 5.3. The belief change operator * used in Example 17 satisfies the supplementary AGM revision postulates.

Theorem 5.5. There is a non-monotonic inference relation that satisfies the supplementary nonmonotonic axioms, but violate some of the basic AGM revision postulates.

Theorem 5.5 shows us that even in the presence of the supplementary axioms, it is not possible to establish the desired connection between AGM revision postulates and non-monotonic inference axioms without the compactness assumption. This naturally leads to the question whether there are conditions under which the revision operation induced by a rational inference relation $\sim$ is AGM-rational in absence of compactness. This is the issue we examine in the next section. 


\subsection{Bridging the gap}

We have seen that the basic axioms of non-monotonicity alone are not strong enough to capture all the basic AGM revision postulates. We also saw that resorting to the supplementary axioms does not help in capturing the basic AGM revision postulates either, let alone the supplementary AGM revision postulates.

In this section, we will show that it is possible to connect AGM revision and non-monotonic inference. We will also show what conditions are needed to establish such a connection. Towards this end let us first identify the largest set of AGM postulates that are induced by the basic axioms of non-monotonicity.

Let us start with the easy ones. Axiom N2 corresponds to $\left(\mathbf{K}_{6}^{*}\right)$ (extensionality), and says that if two formulae are logically equivalent modulo $C n$, then they entail the same set of formulae. The success postulate $\left(\mathbf{K}_{2}^{*}\right)$ is captured by the axiom N1. The following theorem shows that postulate $\left(\mathbf{K}_{5}^{*}\right)$ is jointly captured by axioms $\mathbf{N} 7$ and $\mathbf{N} 1$.

Theorem 5.6. Let $\sim$ be an inference relation and $*$ its induced belief change operator. Then:

(i) if $\sim$ satisfies $\mathbf{N 1}$, then $*$ satisfies $\left(\boldsymbol{K}_{2}^{*}\right)$ (success);

(ii) if $\boldsymbol{\sim}$ satisfies $\mathbf{N} 2$, then $*$ satisfies $\left(\boldsymbol{K}_{6}^{*}\right)$ (extensionality);

(iii) if $\sim$ satisfies both $\boldsymbol{N}^{\boldsymbol{7}}$ and $\mathbf{N} \mathbf{1}$ then $*$ satisfies $\left(\boldsymbol{K}_{5}^{*}\right)$ (consistency).

So far, of the basic AGM postulates, the ones that are missing are $\left(\mathbf{K}_{1}^{*}\right),\left(\mathbf{K}_{3}^{*}\right)$ and $\left(\mathbf{K}_{4}^{*}\right)$. As we will see, the basic axioms of non-monotonicity are not strong enough to capture these three AGM postulates. We start with ( $\left.\mathbf{K}_{1}^{*}\right)$ (the closure).

As we discussed in Section 5.1, when the underlying logic $C n$ is compact, axioms N3 and N4 trivially imply Closure $_{\mathrm{NM}}$. However, when $C n$ is not assumed to be compact, N3 and N4 may no longer guarantee Closure $_{\mathrm{NM}}$. Consequently, a non-monotonic inference may fail to induce a belief change operator $*$ that satisfies $\left(\mathbf{K}_{1}^{*}\right)$. Therefore, satisfaction of $\left(\mathbf{K}_{1}^{*}\right)$ necessitates the presence of the Closure $_{\mathrm{NM}}$ axiom.

Proposition 5.1. An inference relation $\sim$ satisfies Closure $_{\mathrm{NM}}$ if and only if the belief change operator $*$ induced by it satisfies $\left(\boldsymbol{K}_{1}^{*}\right)$.

One question that immediately arises is: Do we still need N3 and N4 in presence of Closure $_{\mathrm{NM}}$ ?

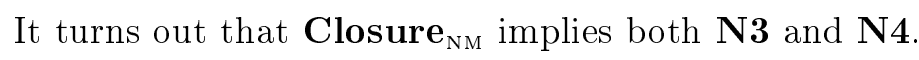

Proposition 5.2. Closure $_{\mathrm{NM}}$ implies N3 and N4.

Now we analyse the other two missing postulates: $\left(\mathbf{K}_{3}^{*}\right)$ and $\left(\mathbf{K}_{4}^{*}\right)$. In Section 5.3, we showed in Example 17 that non-monotonic inference relations may fail to induce postulate $\left(\mathbf{K}_{3}^{*}\right)$. In that example, reasoning from a tautological evidence allowed the inference of new information not previously believed: recall that $C n(\top) * \top$ entailed the propositional symbol $\mathrm{p}$ which is clearly not in $C n(\top)$.

This means that, the axioms do not forbid a non-monotonic inference to augment its body of knowledge in the light of a tautology. In other words, if a formula $\varphi$ does not belong to a theory 
$K$, it is possible that $\top \sim \varphi$. So, our first task is to prevent such inappropriate acquisition of information. Hence we propose the following condition:

(Keeper) if $K$ is consistent and $\sim \varphi$ then $\varphi \in K$.

This allows us to capture postulate $\left(\mathbf{K}_{3}^{*}\right)$.

Proposition 5.3. Let $\sim$ be an inference relation. If it satisfies both $\mathbf{N 5}$ and the Keeper, then the belief change operator $*$ induced by it satisfies $\left(\boldsymbol{K}_{3}^{*}\right)$.

Proof. Let $\sim$ be an inference relation that satisfies both N5 and Keeper. We will show that its induced belief change operator $*$ satisfies $\left(\mathbf{K}_{3}^{*}\right)$, that is, $K * \varphi \subseteq K+\varphi$. Let $\alpha$ be a formula in $K * \varphi$. It follows then that $\varphi \sim \alpha$. Then it follows from N5 that $\sim \varphi \rightarrow \alpha$. Thus, from the Keeper, we get that $\varphi \rightarrow \alpha \in K$. Therefore, $\alpha \in K+\varphi$.

Though Keeper is strong enough to capture $\left(\mathbf{K}_{3}^{*}\right)$, it fails to capture postulate $\left(\mathbf{K}_{4}^{*}\right)$ even in the presence of the seven basic axioms of non-monotonicity. To notice that, let us take the Example 18 below.

Example 18. Let $\otimes$ be a fully AGM rational revision function. We construct the following belief change operation:

$$
K * \varphi=\left\{\begin{array}{lc}
C n(\varphi) & \text { if } \neg \varphi \notin K \\
K \otimes \varphi & \text { otherwise }
\end{array}\right.
$$

Example 18 is similar to Example 17 except that when the input evidence is consistent with the current knowledge, the agent believes the information contained in that evidence alone (and forgoes all the old information). Clearly ${ }^{*}$ so defined violates $\left(\mathbf{K}_{4}^{*}\right)$. We will show that $*$ induces an inference relation $\sim$ that satisfies all basic non-monotonic inference axioms and the Keeper.

Proposition 5.4. Satisfaction of $\left(K_{4}^{*}\right)$ by a revision operation * is not necessary to induce an inference relation $\sim$ which satisfies all basic axioms of non-monotonicity.

The next step deals with Keeper.

Proposition 5.5. The belief change operation used in Example 18 induces an inference relation $\sim$ that satisfies the Keeper.

Proof. Let us suppose that $\sim \varphi$, we need to show that $\varphi \in K$. As $\sim$ is induced from $*$, we have that $\varphi \in K * \top$. We have two cases to consider $K * \top=K \otimes \top$ or $K * \top=C n(\top)$.

For $K * \top=K \otimes \top$, as $\otimes$ satisfies all the basic AGM revision postulates, we have from $\left(\mathbf{K}_{3}^{*}\right)$ that $K * \top \subseteq K$, thus $\varphi \in K$.

For $K * \top=C n(\top)$ we have that $\varphi \in C n(\top)$, which implies that $\varphi \in K$, as $K$ is a theory.

Example 18 helps to show that adding the Keeper is not enough to capture the $\left(\mathbf{K}_{4}^{*}\right)$. In that example, the main reason that $\left(\mathbf{K}_{4}^{*}\right)$ failed is that we are allowing loss of information. This suggests that we should avoid losing formulae when the input formula is consistent with a theory $K$. Hence we introduce a further condition: 
(Rooting) if $K$ is consistent and $\varphi \in K$ then $\sim_{K} \varphi$.

The Rooting axiom is the converse of the Keeper and enforces that the formulae present in a theory $K$ should remain in the non-monotonic inferences by a tautology. The Rooting is the last piece of the puzzle to our first side of the representation theorem. The Rooting together with the Keeper and $\mathbf{N} \mathbf{6}$ captures $\left(\mathbf{K}_{4}^{*}\right)$.

Proposition 5.6. If an inference relation $\sim$ satisfies Keeper, Rooting and $\mathbf{N 6}$, then its induced belief change operator $*$ satisfies $\left(\boldsymbol{K}_{4}^{*}\right)$.

Proof. Let $\sim$ be an inference relation that satisfies Keeper, Rooting and N6, and $*$ its induced belief change operator. We will show that $*$ satisfies $\left(\mathbf{K}_{4}^{*}\right)$. To show this, let us suppose that $\neg \varphi \notin K$, we need to show that $K+\varphi \subseteq K * \varphi$. In other words, for every formula $\alpha \in K+\varphi$ we need to show that $\alpha \in K * \varphi$. By hypothesis, $\neg \varphi \notin K$ which from the contrapositive of the Keeper implies that $\mathcal{L} \neg \varphi$.

As $\alpha \in K+\varphi$, we have that $\varphi \rightarrow \alpha \in K$ from Observation 1. Thus, from Rooting $\sim \varphi \rightarrow \alpha$. Thus we have that both $\mathcal{L} \neg \varphi$ and $\sim \varphi \rightarrow \alpha$. Thus, from $\mathbf{N} 6$ we have that $\varphi \sim \alpha$ which implies that $\alpha \in K * \varphi$.

Now we have all the pieces of the puzzle to show the first result of the representation theorem, that is, the basic non-monotonic axioms augmented with the Keeper and Rooting induces AGM rational revision operators.

Theorem 5.7. Let $\sim$ be a non-monotonic inference relation, if it satisfies the Keeper, Rooting and Closure $_{\mathrm{NM}}$ then its induced belief revision operator $*$ is AGM rational.

Proof. From Propositions 5.3 and 5.6 we have that $*$ satisfies $\left(\mathbf{K}_{3}^{*}\right)$ and $\left(\mathbf{K}_{4}^{*}\right)$. Moreover, from Theorem 5.6 we have $\left(\mathbf{K}_{2}^{*}\right),\left(\mathbf{K}_{5}^{*}\right)$ and $\left(\mathbf{K}_{6}^{*}\right)$. Finally, from Proposition 5.1 we have that Closure ${ }_{\mathrm{NM}}$ implies $\left(\mathbf{K}_{1}^{*}\right)$. This finishes the proof.

So far, we were able to show one direction of the representation theorem. However, as we have included two new axioms to the systems, we need to show that rational AGM revision operators induces non-monotonic inferences that satisfy, besides the basic axioms, the Keeper, Rooting and the Closure $_{\mathrm{NM}}$.

Theorem 5.8. An AGM rational belief revision operator induces a non-monotonic inference that satisfies Rooting, Keeper and Closure $_{\mathrm{NM}}$.

Proof. Let $*$ be an AGM rational operator, that is, it satisfies the six basic AGM revision postulates. We have from Theorem 5.2 that it induces a non-monotonic inference relation $\sim$. We will show that it also satisfies Rooting, Keeper and Closure $_{\mathrm{NM}}$. For Keeper and Rooting, let $K$ be a consistent theory. From $\left(\mathbf{K}_{3}^{*}\right)$ and $\left(\mathbf{K}_{4}^{*}\right)$, we have that

$$
K * \top=K .
$$


As a formula $\varphi \in K * \psi$ iff $\psi \mid \sim \varphi$, we have then from (5.1) that $\varphi \in K * \top$ iff $\sim \varphi$. This means that $\sim$ satisfies both Keeper and Rooting. Furthermore, since ${ }^{*}$ satisfies $\left(\mathbf{K}_{1}^{*}\right)$, it follows from the contrapositive of Proposition 2 that $\sim$ satisfies the Closure $_{\mathrm{NM}}$.

We reached our first representation theorem, in the form of Corollary 5.3 below, which comprises Theorems 5.7 and 5.8. This result is due to that adding Keeper, Rooting and Closure $\mathbf{N M}_{\mathrm{N}}$ axioms to the basic set of non-monotonic inference axioms stablishes a bridge between AGM belief revision operators and non-monotonic logics.

Corollary 5.3. A belief change operator $*$ is AGM rational iff its induced inference relation $\sim$ is a non-monotonic inference relation that satisfies Closure $_{\mathrm{NM}}$, Keeper and Rooting.

Proof. It is straightforward from Theorems 5.7 and 5.8.

\subsubsection{Capturing Supplementary Postulates}

Our first representation result comprises Corollary 5.3, which states that a non-monotonic inference relation augmented with the Keeper and Rooting induce an AGM rational belief revision operators, and AGM rational belief revision operators also induce non-monotonic inference relations that satisfy, besides the basic axioms, the Keeper and Rooting.

The next representation theorem concerns the supplementary postulates and the supplementary axioms. We start showing that axioms N8 and N9 induce belief change operators that satisfy ( $\left.\mathbf{K}_{7}^{*}\right)$ and $\left(\mathbf{K}_{8}^{*}\right)$ respectively.

Theorem 5.9. If a non-monotonic inference relation $\sim$ satisfies $\mathbf{N} 8$ then the belief change function * induced by it satisfies $\left(\boldsymbol{K}_{7}^{*}\right)$.

Proof. Given a formula $\alpha \in K * \varphi \wedge \psi$, we need to show that $\alpha \in K * \varphi+\psi$. As $\alpha \in K * \varphi \wedge \psi$, we have that $\varphi \wedge \psi \sim \alpha$ which from N8 implies that $\varphi \sim \psi \rightarrow \alpha$. This means that $\psi \rightarrow \alpha \in K * \varphi$. Thus, $\alpha \in K * \varphi+\psi$.

Theorem 5.10. If an inference relation $\sim$ satisfies both $\mathbf{N 9}$ and Closure $_{\mathrm{NM}}$, then its induced belief change operation $*$ satisfies $\left(\boldsymbol{K}_{8}^{*}\right)$.

Proof. Let us suppose that $\neg \psi \notin K * \varphi$, the we need to show that $K * \varphi+\psi \subseteq K * \varphi \wedge \psi$. In other words, given a formula $\alpha \in K * \varphi+\psi$, we need to show that $\alpha \in K * \varphi \wedge \psi$. From $\neg \psi \notin K * \varphi$, we have that $\varphi \not \mathcal{} \neg \psi$. Moreover, as $\sim$ satisfies Closure $_{\mathrm{NM}}$, we have that both $K * \varphi$ and $K * \varphi \wedge \psi$ are theories. Therefore, $\alpha \in(K * \varphi)+\psi$ implies Observation 1 that $\psi \rightarrow \alpha \in K * \varphi$, which means that

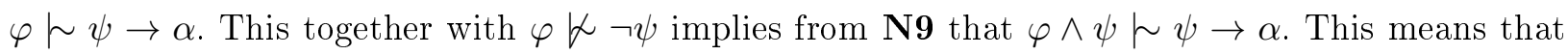
$\psi \rightarrow \alpha \in K * \varphi \wedge \psi$. Thus, as $\psi \in K * \varphi \wedge \psi$, we have that $\alpha \in K * \varphi \wedge \psi$.

This second representation result, an immediate consequence from Corollary 5.3, together with Theorems 5.9 and 5.10, shows that $\mathbf{N} 8$ and $\mathbf{N 9}$ translates respectively into $\left(\mathbf{K}_{7}^{*}\right)$ and $\left(\mathbf{K}_{8}^{*}\right)$, and vice-versa.

Corollary 5.4. A belief change operator $*$ is fully AGM rational iff its induced inference relation $\sim$ satisfies all the basic, and supplementary axioms of non-monotonicity together with Keeper and Rooting. 
We know from Proposition 5.2 that N3 and N4 are superfluous in presence of Closure $_{\mathrm{NM}}$. Therefore, we may assume a shorter set of non-monotonic axioms: N1, N2, N5 to N7 and the Keeper, Rooting and Closure $_{\mathrm{NM}}$. Hence the Corollary 5.3 can be stated more simply as:

Corollary 5.5. A belief change operator $*$ is AGM rational iff its induced inference relation $\sim$ satisfies N1, N2, (N5 - N7), Keeper, Rooting and Closure NM $_{\text {. }}$.

\subsection{Summary and the Highlights}

In this chapter we have addressed the connection between AGM revision operators and nonmonotonic logics. Unlike the previous works, we no longer assume compactness. We showed that dropping compactness has some immediate consequences on the non-monotonic system. For in-

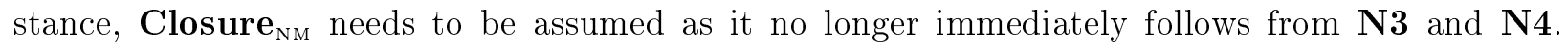
Moreover, in presence of Closure $\mathbf{N M}_{\mathrm{NM}}, \mathbf{N 3}$ and N4 become redundant. Another two consequences is that, though the direction from AGM revision postulates to the non-monotonic system we addressed remains untouched, the converse is not true. We showed that the postulates $\left(\mathbf{K}_{3}^{*}\right)$ and $\left(\mathbf{K}_{4}^{*}\right)$ are violated in this side of the trip. To solve this problem, we studied which axioms remained as a translation of the AGM postulates, and then we proposed two new axioms, the Keeper and Rooting, in order to strength the non-monotonic system to be able to reconnect with AGM paradigm.

At the end, we were able to show a connection between the enhanced non-monotonic system and the AGM belief postulates: both for the basic postulates as well as the supplementary postulates. One more difference between our work and the classical one is that whereas the study between AGM revision and non-monotonic inference rides on specific belief revision operators such as partial meet or other constructions, our proofs navigate directly from postulates to axioms and vice-versa without the need to assume any specific construction. This makes the study more general, as we assume very little about the underlying logics, namely, to be only Tarskian and closed under classical negation and disjunction. 


\section{Chapter 6}

\section{Concluding Remarks}

In this thesis, we have addressed the issue of Belief Change for non-compact logics. For this, we considered the two foremost influential works of belief change: the AGM paradigm of belief revision and the KM paradigm of belief update. Towards this end, we have examined the connection between belief revision and non-monotonic logics when the requirement of the underlying logic to be compact is dispensed with. We outline the main contributions of this work in Section 6.1. In the subsequent section, Section 6.2, we discuss the directly impact of this work has on AI and computer science. We then discuss some research directions we aim to follow in future, as well as some interesting questions to be pursued.

\subsection{Main Contributions}

This work can be split into three main categories based on its results: AGM paradigm, KM paradigm, and non-monotonic reasoning versus AGM belief revision. We outline the main results of this work following this classification.

\section{AGM paradigm and non-compact logics}

Efforts to extend belief change to non-compact logics is difficult. In the AGM paradigm of belief change, for instance, the connection between the standard constructions of belief change and the AGM rationality postulates ceases to exist (Ribeiro et al., 2013). Even worse, Guerra (2016) has shown that it is not even possible to define Partial Meet functions (one of the most fundamental AGM constructions) in some non-compact logics, such as the temporal logics LTL and CTL. Against this backdrop, we posed the following question:

\section{Can we construct AGM rational functions without assuming compactness?}

In answering this question, we were able to identify a class of logics that are AGM compliant independent of compactness: logics that are at least closed under classical negation and disjunction. Thereafter, we proposed classes of (fully) AGM rational contraction and revision functions. We split these functions into two main classes: basic ones, concerned to the basic AGM postulates, and the fully AGM rational functions (considering the supplementary postulates). Contrary to the standard AGM rational constructions, the functions we proposed do not depend on total relations. 
An interesting aspect about AGM contraction and revision is that they are inter-definable via the Levi and Harper identity. We proved that both contraction and revision remain inter-definable via these identities as long as the logic in question is closed under classical negation and disjunction. Summarising, the main results of this work for AGM belief change are:

1. determining an AGM compliant class of logics: logics closed under classical negation and disjunction;

2. new classes of (fully) AGM rational functions (both contraction and revision) for the class of logics identified in item (1);

3. AGM rational functions independent of total relations: the functions are defined over general binary relation that requires only two essential requirements: maximal-cut, and mirroring;

4. proof that the inter-definability between contraction and revision, via the Levi and the Harper identities, does not depend on compactness;

To sum up, we have provided complete accounts of AGM belief change for non-compact logics, assuming very little about the underlying logics, other than closure under classical negation and disjunction.

\section{KM update in non-finatary logics without compactness}

Our investigations towards the AGM paradigm proved very fruitful. In order to provide full accounts of belief change, we decided to investigate what would be the consequences of dispensing with compactness in the KM paradigm of belief update. As expected, the standard constructions of belief update are not strong enough to capture all the KM update rationality postulate. Perhaps some what surprisingly, we found that the source of the problem was not compactness itself, but the non-finitary property. As the lack of compactness implies in non-finitariness, we decided to dispense with both properties. This allowed us to construct a class of KM rational update function for these logics, only requiring the underling logic to be closed under classical negation and disjunction. In the converse direction, we investigated as to what postulates the standard update functions would connect with, leading to a new class of postulates for these functions. These new postulates, which is generalization of the original ones, consider explicitly a side-effect of decomposing formulae in nonfinitary logics: that the decomposition of a finite formula may yield an infinity number of formulae that are not logically equivalent. As it turned out, the original supplementary postulates of KM update depends on a finitary aspect of the background logics, that is, the original postulates are too fragile to deal with non-finitary logics. This is an interesting result, since it tell us more about the behaviour of the standard update functions, and gives us tools to better understand the behaviour of such postulates and their connection with finitariness (or the lack of it). In summary, our main contributions involving KM update are:

1. A new class of fully KM-rational update functions for logics closed under classical negation and disjunction, independent of comapctness or finitariness.

2. identification of the rationality postulates for the standard KM update function for nonfinitary and non-compact logics. 


\section{AGM paradigm and Non-monotonic reasoning}

Non-monotonic reasoning and Belief Change are arguably to be two perspectives of the same phenomenon. Indeed, when the underlying logic satisfies the AGM assumption, the Non-monotonic system based on expectation ordering and the AGM revision are equivalent, in the sense that each AGM rational belief revision function corresponds to a non-monotonic entailment relation based on expectation, and vice-versa. This correspondence relies strongly on the standard AGM constructions. Since these constructions do not behave as usual in the absence of compactness, it is natural to ask what happens to the connection between AGM revision and the non-monotonic systems. On the negative side, we have identified that this connection breaks down. On the positive side, we were able to restore this bridge by defining a new non-monotonic reasoning system. In particular, we have introduced two new axioms to the non-monotonic system based on expectation. The resultant non-monotonic system connects with the AGM revision. An interesting aspect of our result is that we do not depend on a specific class of AGM rational constructions. Instead, our proof depends on a direct connection between the AGM revision postulates and the new non-monotonic system axioms. This is important, since it helps to understand how these two systems behave.

\subsection{Discussion and Future Work}

This thesis provides complete accounts of belief change, both AGM and KM styles, for logics that are not compact, therefore non-finitary. Many different disciplines within AI and computer science will benefit directly from the achievements of this thesis. We explore in the rest of this section such directly impact, as well as some immediate questions we aim to address very soon.

\section{The Relevance Postulate, and the Standard AGM Constructions}

The recovery postulate is argued to be the only one, among the basic AGM contraction postulates, to give a notion of minimal change. However, it is very controversial and has trigged many discussions in the literature; mainly because it behaves in a counter-intuitive way in some scenarios (see Chapter 2.3). The relevance postulate was proposed as an alternative to the recovery postulate. First investigations towards the role of compactness and the relevance postulate was initially address by Ribeiro et al. (2013). However, it is not clear yet how non-compact logics connects with relevance postulate. This is an interesting topic worth exploring in the future. Departing from the results achieved in this thesis and the initial considerations explored by Ribeiro et al., we will investigate the consequences of the relevance postulate by dispensing with compactness, and identify which are the class of relevance-rational construction in non-compact logics.

There is yet another aspect of the AGM paradigm that deserves some attention. With respect to the AGM approrach, we fixed the AGM rationality postulates and found a class of functions that is characterised exactly by such postulates. Although this tell us how to perform belief change on non-compact logics, taking the AGM postulates for granted, it does not tell us how the standard AGM constructions behave in non-compact logics. Towards this end, we intend to identify a set of rationality postulates that emerge from the standard AGM constructions in logics that lack compactness. Moreover, we will also address the consequences of relying in this set of postulates 
and standard constructions. This will facilitate a more complete understanding of AGM belief change without heavy reliance on classical assumptions of the underlying logic.

\section{A New Perspective on KM Paradigm}

There are two main forms of belief change in the KM paradigm: update and eraser. So far, we have considered only KM update in the context of non-compact and non-finitary logics. Although erasure is originally defined via Harper's Identity from the CUP functions (the standard update functions), it is possible that the functions yielded via Harper's identity from splinters functions and CUP functions, in non-compact (and non-finitary) logics, do not satisfy the original KM erasure postulates. There are two reasons that support this point of view: first, the CUP functions do not connect with the original KM update postulates; secondly, the original KM update postulates present some issues to capture their original purpose, since postulate $(\mathrm{K} \diamond 8)$ is not strong enough to sustain various decomposition of finite formulae in the context of non-finitary logics.

We have then two main paths to pursue KM update on non-finitary logics without compactness: (i) apply Harper identity on the Royal Splinter functions we defined, and investigate if, and if so how, the class of functions so obtained connects with the original KM erasure postulates; or (ii) similar to what we did for update, one can analyse the class of the original erasure functions and identify which rationality postulates emerge from them.

We have another issue regarding KM erasure. We showed that the KM update supplementary postulates are too weak in the absence of compactness, identified the reason of such weakness (related to the transitivity of underlying preference relations) and proposed a stronger version. We expect that the supplementary postulate for erasure will suffer from analogous issues. We expect that our finding regarding the supplementary postulates for update can be adapted for erasure.

The KM update was originally proposed to deal with knowledge specified in Proposition Logic. Extending such a paradigm to more expressive logics can be a challenge since the standard constructions do not behave as expected. A natural question to pose then is: can KM rational update functions be defined in every logic, and if not in which logics we can build such functions? This issue occurs in the AGM paradigm, and Flouris (2006) has identified the necessary and sufficient conditions for the existence of AGM rational contraction functions in a logic. It is then worth investigating which logics admits KM rational functions. Due to the similarity between AGM and KM, one would expect that AGM-compliance and KM-compliance would simply collapse to the same set of conditions. However we believe it may not be the case due to Gärdenfors impossibility theorem. This issue merits our future investigation.

\section{Another look at Quasi-reflection}

The class of update functions we defined here triggers some interesting and perhaps deeper questions. The most interesting one is possibly the quasi-reflection property we introduced which poses as the key to capture the supplementary KM update rationality postulates. Quasi-reflection is rather intriguing since it is a relaxation of the mirroring property that we defined in order to capture the AGM rationality postulates (both contraction and revision). This relaxation consists of, so to speak, pushing up the mimicry of two equally preferable elements one level up. This suggests 
that there is a deeper connection between mirroring and quasi-reflection with respect to preference specification.

Quasi-reflection could be used to conceptualise the notion of indifference and ignorance on preference relations. These concepts play important roles in many area of scholarship. For instance, in the context of preference and choice, an agent is indifferent between two items $x$ and $y$ if they are equally preferable,

Equipreference: $x$ ind $y$ if and only if $x \leq y$ and $y \leq x$.

On the other hand, an agent could be indifferent between two items if there is no discernible preference between them - the two items are incomparable as far as the agent's preference is concerned. Under an epistemic reading, incomparability may be interpreted as ignorance.

Ignorance: $x$ ind $y$ if and only if $x \not \leq y$ and $y \not \leq x$.

In the context of probability, the Principle of Indifference essentially says that if we are ignorant about the probability of outcomes in a certain space, we should assign them equal probability. Indeed the Principle of Indifference was historically called the Principle of Insufficient Reason.

Thus the two notions, indifference and ignorance are conflated. However we might be able to establish a more nuanced connection between these two notions by introducing a notion of graded indifference - that there are different levels of indifference. At the strongest level, indifference corresponds to equal preference (analogous to equiprobable events); at the weaker levels, indifference is captured by incomparability (or ignorance) coupled with certain aspects of equal-preference.

A rough definition of graded indifference is given below:

Definition 6.1. GRADED Indifference

$$
\begin{aligned}
& x \text { ind }{ }^{0} y \text { iff } x \leq y \text { and } y \leq x \\
& x \text { ind }{ }^{1} y \text { iff }\left\{\begin{array}{l}
x \not \leq y \text { and } y \not \leq x \\
x \leq y_{1} \text { and } y \leq x_{1} \quad \text { if } x \leq x_{1} \text { and } y \leq y_{1}
\end{array}\right. \\
& x \text { ind }{ }^{n+1} y \text { iff }\left\{\begin{array}{lll}
x \not \leq y^{i} \text { and } y \not \leq x^{i} & \text { for } & 0 \leq i \leq n \\
x \leq y_{n+1} ; y \leq x_{n+1} & \text { if } & x \leq x_{1} \leq \cdots \leq x_{n+1} \\
& & y \leq y_{1} \leq \cdots \leq y_{n+1}
\end{array}\right.
\end{aligned}
$$

It should be clear that indifference of level 0 corresponds to equal-preference, indifference of level 1 corresponds to mirroring, and indifference of level 2 will corresponds to what we call quasireflection. This opens up further avenues for exploring deeper connection between belief change and rational choice theory.

\section{Towards Iterated Belief Change}

The new classes of functions we introduced, precisely the Blade and Constellar functions, present some peculiar properties when compared to the standard AGM rational belief change functions, such as the System of Spheres and the Epistemic Entrenchment functions. The most notable one 
is that the both Blade and Constellar functions do not require the preference relation of an agent to be centred on an belief set $K$ (the current agent's epistemic state), whereas such a centring requirement is present on the standard AGM constructions. Although this can be viewed as a mere technical issue, a good understanding of why such a requirement can be waived could help us better understand the behaviour of AGM rational constructions.

An interesting path to follow here concerns iterated belief change. Since we do not require a preference relation to be centred on a specific epistemic state, can Constellar/Blade functions be used in the context of iterated belief change? Will we need to specify some kind of general preference relation? We already know how to perform one-shot belief change for non-compact and non-finatary logics. It remains to be seen how iterated versions of such change operations behave.

\section{Non-compact Dynamic Logics}

Dynamic logics such as Dynamic Epistemic Logics (DEL) and Dynamic Doxastic Logics (DDL) are used to reason about the dynamics involved in changing an agent's belief or knowledge. Whereas belief change approaches such as AGM and KM paradigm conceptualise the dynamism as an extra logical feature; DDL and DEL capture these dynamics in the object language via modal operators. A limitation of these Dynamic Logics is that they can only conceptualise Knowledge/Beliefs as information expressed in propositional logic. More expressive information, such as temporal reasoning, cannot be expressed in these logics. This represents a severe limitation when we look at some sub-disciplines in AI, such as planning, where conceptualising and modelling temporal knowledge is fundamental.

We are optimistic that the results of this thesis can be extended and adapted to provide a new semantics for Dynamic logics in the context of non-compact logics. Both from KM and AGM that extends belief change for non-compact logics, give us the first apparatus to devise new semantics for Dynamic logics in the context of non-compact logics. This is line of research worth to explore in the future.

\section{Planning and Goal Revision/Update}

Planning is a sub-discipline in AI that focuses in tracing a chain of actions an agent has to perform in order to complete a task. One of the most important component in Planning is goal specification. An agent usually needs to find a plan (a chain of actions) that guarantees to reach the desired goal. In a dynamic world, however, an agent might need to consider the dynamics of the environment in order to choose its actions towards its goal. This leads to two main issues: (1) the environment might change in a way that an agent needs to find another plan to perform its task and keep working towards its goal, or (ii) the change on the environment is so drastic that it is not possible any more for the agent to fulfil the initial goal. The former leads to re-planning its actions, whereas in the latter an agent might consider relaxing it is goal in order to fulfil its tasks. Ideally, an agent should be able to automatically re-plan or revise its goals. We aim to investigate, in the future, techniques of combining belief change and temporal reasoning, which is widely used in planning to express actions and agent's knowledge, in order to provide enhanced techniques for revising goal and plans. Since Dynamic logics also can be used to deal with planning, this new line 
of proposed research aligns with the non-compact dynamic logics that we outlined earlier. 
CONCLUDING REMARKS 


\section{Appendix A}

\section{Proofs for Chapter 3, Section 3.1 to 3.3}

Note: We will write $M=\varphi$ to denote that a model $M$ satisfies a formula $\varphi$, and we will also write, for convenience, $M \models X$ to denote that $M$ satisfies all formulae of a set of sentences $X$.

Observation 3.1. Let $K$ be a theory, and $A$ a proper subset of $K$ such that $C n(A) \subset C n(K)$. Moreover, let $Y=[K] \cup\{M\}$, where $M$ is an interpretation. If $M \forall \varphi$, then for every formula $\alpha \in K$, there is some $\varphi \in A$ such that $\varphi \rightarrow \alpha \in \operatorname{Th}(Y)$.

Proof. Let $\alpha$ be a formula from $K$, we will show that $\varphi \rightarrow \alpha \in T h(Y)$, for some formula $\varphi \in A$. As $Y=\llbracket K \rrbracket \cup\{M\}$, where $M \not \models \varphi$, we get that for some formula $\varphi \in A, M \not \models \varphi$. Let $M^{\prime} \in Y$, we have then that either (i) $M^{\prime} \in \llbracket K \rrbracket$ or $M^{\prime}=M$. Let us consider each case separately:

(i) $M \in \llbracket K \rrbracket$. Thus, $M \models \alpha$ which implies that $M \models \varphi \rightarrow \alpha$.

(ii) $M=M^{\prime}$. Thus, $M \not \models \varphi$ which implies that $M \models \varphi \rightarrow \alpha$.

From (i) and (ii) we conclude that each model $M$ from $Y$ satisfies $\varphi \rightarrow \alpha$. Therefore, $\varphi \rightarrow \alpha \in$ $T h(Y)$.

Observation 3.2. Given a theory $K, T h(\llbracket K \rrbracket)=K$.

Proof. We need to show that a formula $\alpha$ is in $K$ iff $\alpha \in T h(\llbracket K \rrbracket)$. From the definitions of $T h$ and 【』, we have that

(i) $\alpha \in K$ iff $M \models \alpha$, for every $M \in \llbracket K \rrbracket$;

(ii) $\alpha \in T h(\llbracket K \rrbracket)$ iff $M \models \alpha$, for every $M \in \llbracket K \rrbracket$.

Thus, from (i) and (ii) we get that $\alpha \in K$ iff $\alpha \in T h(\llbracket K \rrbracket)$.

Proposition 3.1. Let $X$ and $Y$ be two sets of interpretations. If $X \subseteq Z$ then $T h(Z) \subseteq T h(X)$.

Proof. Let $\alpha$ be a formula in $T h(Z)$, we will show that $\alpha \in T h(X)$. From $T h$ definition, we have that $\alpha \in T h(Z)$ iff $M \models \alpha$, for every model $M \in Z$. Thus, as $X \subseteq Z$, we get $M \models \alpha$, for every $M \in X$. This implies that, $\alpha \in T h(X)$.

Theorem 3.1. Every Tarskian logic closed under classical negation and disjunction is AGMCompliant. 
Proof. Let $K$ be a theory and $A$ a proper subset of $K$ such that $C n(A) \neq C n(\emptyset)$. We will show that there is a relative complement $K^{\prime}$ of $K$ w.r.t $A$. Precisely, we will show that (i) $C n\left(K^{\prime}\right) \subset K$ and (ii) $C n\left(A \cup K^{\prime}\right)=K$.

As $A \neq C n(\emptyset)$, we get that $A$ has at least one non-tautological formula $\psi$. Thus, there is at least a model $M$ that does not satisfies $\psi$ and therefore $M$ does not satisfies $A$. We proceed with our schemata explained in Chapter 3. Let us make

$$
Y=\llbracket K \rrbracket \cup\{M\},
$$

and let $K^{\prime}=T h(Y)$. We proceed to show that we have the items (i) and (ii):

(i) $C n\left(K^{\prime}\right) \subset K$. As $Y \subseteq \llbracket K \rrbracket$, we get from Proposition 3.1 and Observation 3.2 that $T h(Y) \subseteq$ $K$. Since, $M$ does not satisfy $A$, we have that for at least one formula $\varphi \in A, M \forall \varphi$ which implies that $\varphi \notin T h(Y)$. Notice that as $A \subset K$, we get that $\varphi \in K$. Thus, $T h(Y) \subset K$, and since $T h(Y)=K^{\prime}$, we get $K^{\prime} \subset K$.

(ii) $C n\left(A \cup K^{\prime}\right)=K$. We need to show that (a) $K \subseteq C n\left(A \cup K^{\prime}\right)$ and (b) $C n\left(A \cup K^{\prime}\right) \subseteq K$. Since both $K^{\prime}$ and $A$ are subsets of $K$, we get immediately from the monotonicity of $C n$ that (b) $C n\left(K^{\prime} \cup A\right) \subseteq K$. We focus then only on proving item (a). For this, let $\alpha$ be a formula from $K$, we need to show that $\alpha \in C n\left(K^{\prime} \cup A\right)$. From Observation 3.1, we get that there is a formula $\varphi \in A$, such that $\varphi \rightarrow \alpha \in T h(Y)=K^{\prime}$. Thus, both $\varphi$ and $\varphi \rightarrow \alpha$ are in $C n\left(K^{\prime} \cup A\right)$ which implies that $\alpha \in C n\left(K^{\prime} \cup A\right)$.

From (i) and (ii) we have that $T h(Y)=K^{\prime}$ is a relative complement. Therefore, logics closed under classical negation and disjunction are AGM compliant.

Lemma A.1. Given a set of formulae $X, \llbracket X \rrbracket=\llbracket C n(X) \rrbracket$.

Proof. We will show that $M \in \llbracket X \rrbracket$ iff $M \in \llbracket C n(X) \rrbracket$.

Recall that

$$
M \in \llbracket C n(X) \rrbracket \text { iff } M \models C n(X) .
$$

As $C n$ is Tarskian, $\alpha \in C n(X)$ iff $M \models \alpha$, for all $M \in \llbracket X \rrbracket$. Thus,

$$
M \in \llbracket X \rrbracket \text { iff } M \models C n(X) .
$$

Therefore, $M \in \llbracket X \rrbracket$ iff $M \in \llbracket C n(X) \rrbracket$.

Observation 3.3. Every $\llbracket X \rrbracket$ is a CIS, where $X$ is a set of formulae.

Proof. Let $X$ be a set of formulae, we will show that $\llbracket X \rrbracket$ is a CIS. Let us suppose for contradiction that $\llbracket X \rrbracket$ is not a CIS. Therefore, there is a model $M^{\prime} \notin \llbracket X \rrbracket$, such that $T h\left(\llbracket X \rrbracket \cup\left\{M^{\prime}\right\}\right) \not \subset T h(\llbracket X \rrbracket)$.

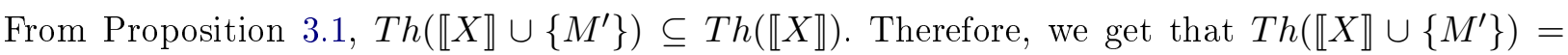


$T h(\llbracket X \rrbracket)$. Moreover, from Lemma A.1

$$
\llbracket X \rrbracket=\llbracket C n(X) \rrbracket,
$$

which implies that $T h\left(\llbracket X \rrbracket \cup\left\{M^{\prime}\right\}\right)=T h(\llbracket C n(X) \rrbracket)$. Thus, from Observation 3.2,

$$
T h\left(\llbracket X \rrbracket \cup\left\{M^{\prime}\right\}\right)=C n(X) .
$$

From the definition of $\llbracket \rrbracket, M \in \llbracket X \rrbracket$ iff $M \models X$. Thus, as $\llbracket X \rrbracket=\llbracket C n(X) \rrbracket$, we get that

$$
M \in \llbracket C n(X) \rrbracket \text { iff } M \models X .
$$

As $M^{\prime} \notin \llbracket X \rrbracket$, we get $M^{\prime} \not \models X$. Thus, as $X \subseteq C n(X)$, we have that

$$
M^{\prime} \not \models C n(X)
$$

From definition of $T h$, we have that

$$
\text { if } M \in Z \text { then } M \models T h(Z) \text {. }
$$

Thus, as $M^{\prime} \in\left(\llbracket X \rrbracket \cup\left\{M^{\prime}\right\}\right)$, it follows that $M^{\prime} \models T h\left(\llbracket X \rrbracket \cup\left\{M^{\prime}\right\}\right)$. Therefore, $M^{\prime} \models C n(X)$ which is a contradiction. Thus, we conclude that $\llbracket X \rrbracket$ is a CIS.

Observation A.1. Let $A$ be a CIS, if $M \notin A$, then $M \not \models T h(A)$.

Proof. Let then $M^{\prime}$ be a model that is not in $A$, we will show that $M \not \models A$. For this we implies in showing that for some formula $\alpha \in A, M \not \models \alpha$. As $A$ is $T h$-dense due to be a CIS, we get

$$
\operatorname{Th}\left(A \cup\left\{M^{\prime}\right\}\right) \subset T h(A) .
$$

From definition of $T h$,

$$
\alpha \in T h(A) \text { iff } M \models \alpha \text {, for all } M \in A \text {. }
$$

As $T h\left(A \cup\left\{M^{\prime}\right\}\right) \subset T h(A)$, it follows that there is some formula $\alpha \in A$ such that $\alpha \notin$ $T h\left(A \cup\left\{M^{\prime}\right\}\right)$. Therefore, for some model $M \in A, M \not \models T h\left(A \cup\left\{M^{\prime}\right\}\right)$. We have from (A.1) that all models of $A$ satisfies $\alpha$, therefore we conclude that the only model that does not satisfies $\alpha$ is $M^{\prime}$. Therefore, $M \not \models \alpha$.

Proposition 3.2. An interpretation set $A$ is a CIS iff $A=\llbracket X \rrbracket$, for some set of formulae $X$.

Proof. $(\Rightarrow)$ Let $A$ be a CIS, and $X=T h(A)$. We will show that $A=\llbracket X \rrbracket$. From Observation A.1 we have that 
We also have from definition of $T h$ that if $M \in A$ then $M \models T h(A)$. This together with the contra-positive of (A.2) above give us that

$$
M \in A \text { iff } M \models T h(A) .
$$

Moreover, from definition of $\llbracket \rrbracket$ we get $M \in \llbracket X \rrbracket$ iff $M \models X$.

As $X=T h(A)$, we get

$$
M \in \llbracket X \rrbracket \text { iff } M \models T h(A),
$$

which implies that $M \in \llbracket X \rrbracket$ iff $M \in A$. Therefore, $A=\llbracket X \rrbracket$.

$(\Leftarrow)$ It is straightforward from Observation 3.3.

Corollary 3.1. Given two $\Theta$-dense sets $A$ and $B$. If $\Theta$ is reverse-monotonic and $A \subset B$, then $\Theta(B) \subset \Theta(A)$.

Proof. We need to show that $\Theta(B) \subset \Theta(A)$. As $A \subset B$, we get there is some $S \in B$, such that $S \notin A$. As $A$ is dense, we have $\Theta(A \cup\{S\}) \subset \Theta(A)$ and $\Theta(A \cup\{S\}) \subseteq B$. Thus, as $\Theta$ is reverse-monotonic we get

$$
\Theta(B) \subseteq \Theta(A \cup\{S\}) \text { and } \Theta(A \cup\{S\}) \subset \Theta(A)
$$

Therefore, $\Theta(B) \subset \Theta(A)$.

Theorem 3.2. Let $\mathcal{T}$ be the the set of all theories from a logic $\langle L, C n\rangle$, and $\mathcal{I}_{C}$ the class of all complete interpretation sets of that logic. Then the function $\tau: \mathcal{T} \rightarrow \mathcal{I}_{C}$ as $\tau(K)=\llbracket K \rrbracket$ is a bijection.

Proof. We have to show that $\tau$ is injective and surjective.

(1) $\tau$ is injective. Let us suppose for contradiction that $\tau$ is not injective, that is, there are theories $K$ and $K^{\prime}$ such that $K \neq K^{\prime}$ and

$$
\tau(K)=\tau\left(K^{\prime}\right)
$$

From definition, we have that for each formula $\varphi$ :

(i) $\varphi \in K$ iff $\tau(K) \models \varphi$, and

(ii) $\varphi \in K^{\prime}$ iff $\tau\left(K^{\prime}\right) \models \varphi$.

As $\tau(K)=\tau\left(K^{\prime}\right)$, we have from (i) that $\varphi \in K$ iff $\tau\left(K^{\prime}\right) \models \varphi$ which together with (ii) implies that $\varphi \in K$ iff $\varphi \in K^{\prime}$. Therefore, $K=K^{\prime}$ which contradicts our assumption. Therefore, $\tau$ is injective. 
(2) $\tau$ is surjective. By definition we have that $\tau(K)=\llbracket K \rrbracket$. Let us suppose for contradiction that $\tau$ is not surjective. Therefore, there is a CIS $A$ such that for all theories $K, \tau(K) \neq A$. In other words, for all theories $K, A \neq \llbracket K \rrbracket$. Let $K=T h(A)$. So, we have

$$
(a) \forall M \in A, M \models K, \quad \text { and } \quad(b) M \models K \text { iff } M \in \llbracket K \rrbracket .
$$

Therefore, from (a) and (b) we get that $A \subseteq \llbracket K \rrbracket$. As from hypothesis $A \neq \llbracket K \rrbracket$, we get that

$$
A \subset \llbracket K \rrbracket .
$$

As $A$ is dense, we have that $T h(\llbracket K \rrbracket) \subset T h(A)$. This implies from Observation 3.2 that $K \subset$ $T h(A)$ which is a contradiction.

Thus, $\tau$ is surjective.

Therefore, from (1) and (2), we conclude $\tau$ is a bijection.

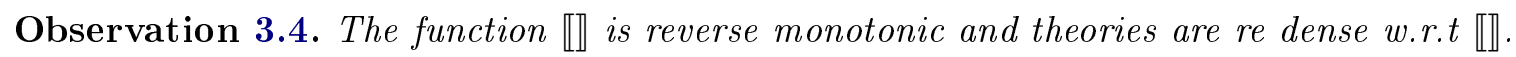

Proof. Recall that the function $\llbracket$ maps each set of formulae (not necessarily a theory) to a set of models. We will proof both statements separately: (i) the function $\llbracket K \rrbracket$ is reverse-monotonic and (ii) theories are dense w.r.t $\llbracket \rrbracket$.

(i) Let $A$ and $B$ be two sets of formulae (not necessarily theories) such that $A \subseteq B$. To show that $\llbracket \rrbracket$ is reverse-monotonic we need to show that $\llbracket B \rrbracket \subseteq \llbracket A \rrbracket$. From definition of $\llbracket \rrbracket$ :

$$
\begin{aligned}
& M \in \llbracket B \rrbracket \text { iff } M=B \text { and } \\
& M \in \llbracket A \rrbracket \text { iff } M \models A .
\end{aligned}
$$

As $A \subseteq B$, we get that if a model $M \models B$ then $M \models A$. This together with the expressions above implies that if $M \in \llbracket B \rrbracket$ then $M \in \llbracket A \rrbracket$. Therefore,

$$
\llbracket B \rrbracket \subseteq \llbracket A \rrbracket .
$$

(ii) To show that theories are dense w.r.t $\mathbb{\rrbracket}$, we need to show that given a theory $K$ and a formula $\varphi \notin K, \llbracket K \cup\{\varphi\} \rrbracket \subset \llbracket K \rrbracket$. From definition of $\llbracket K \rrbracket$ :

$$
\begin{gathered}
M \in \llbracket K \rrbracket \text { iff } M \models K, \text { and } \\
M \in \llbracket K \cup\{\varphi\} \rrbracket \text { iff } M \models K \text { and } M \models \varphi .
\end{gathered}
$$

Let us suppose for contradiction that $\llbracket K \cup\{\varphi\} \rrbracket \not \subset \llbracket K \rrbracket$. As from (i) $\llbracket K \rrbracket$ is reverse-monotonic, we get that $\llbracket K \cup\{\varphi\} \rrbracket \subseteq \llbracket K \rrbracket$. Therefore, $\llbracket K \cup\{\varphi\} \rrbracket=[K]$. As $K$ is a theory, we have that,

$$
\alpha \in K \text { iff } M \models \alpha \text {, for all } M \in \llbracket K \rrbracket \text {. }
$$

As $\varphi \notin K$, it follows that there is a model $M \in \llbracket K \rrbracket$ such that $M \not \models \varphi$. This implies from (A.3) that $M \notin \llbracket K \cup\{\varphi\} \rrbracket$ which is a contradiction. 
Corollary 3.2. The function $\tau$ from Theorem 3.2 is reverse-monotonic.

Proof. Given two theories $K$ and $K^{\prime}$, such that $K \subseteq K^{\prime}$. We need to show that $\tau\left(K^{\prime}\right) \subseteq \tau(K)$. As, from Observation 3.4, the function $\llbracket \rrbracket$ is reverse-monotonic, we get that $\llbracket K^{\prime} \rrbracket \subseteq \llbracket K \rrbracket$. Therefore, as $\tau$ is defined in terms of $\llbracket \mathbb{\rrbracket}$, we get that $\tau\left(K^{\prime}\right) \subseteq \tau(K)$.

Proposition 3.3. Given two theories $K$ and $K^{\prime}, K \subseteq K^{\prime}$ iff $\llbracket K^{\prime} \rrbracket \subseteq \llbracket K \rrbracket$.

Proof. (" $\Rightarrow$ ") We need to show that $K \subseteq K^{\prime}$ implies $\llbracket K^{\prime} \rrbracket \subseteq \llbracket K \rrbracket$ which is straightforward from the reverse-monotonicity of $\llbracket$ 】.

(" $\Leftarrow^{\prime \prime}$ ) Let $\llbracket K^{\prime} \rrbracket \subseteq \llbracket K \rrbracket$. We will show that $K \subseteq K^{\prime}$. From Proposition 3.1, we get that $T h(\llbracket K \rrbracket) \subseteq \llbracket K \rrbracket$. From the bijection between CISs and theories, we have that $T h(\llbracket K \rrbracket)=K$ and $T h\left(\llbracket K^{\prime} \rrbracket\right)=K^{\prime}$. Therefore, $K \subseteq K^{\prime}$.

Proposition 3.4. Let $A$ and $B$ be two CISs, and $K$ and $K^{\prime}$ two theories:

(1) $K \subset K^{\prime}$ iff $\llbracket K^{\prime} \rrbracket \subset \llbracket K \rrbracket$;

(2) $\operatorname{Th}(A) \subset \operatorname{Th}(B)$ iff $B \subset A$.

Proof. We know from Proposition 3.1 and Observation 3.4 that both Th and $\llbracket \rrbracket$ are reversemonotonic. We recall that $\tau$ is defined in terms of $\llbracket \rrbracket$, that is, $\tau(K)=\llbracket K \rrbracket$, for every theory $K$.

(1) ( " $\left.\Rightarrow^{\prime \prime}\right)$ we will show that $K^{\prime} \subset K$ implies $\llbracket K \rrbracket \subset \llbracket K^{\prime} \rrbracket$. As theories are dense with respect to $\llbracket \rrbracket$, and $\llbracket \rrbracket$ is reverse monotonic, it follows from Corollary 3.1 that $\llbracket K \rrbracket \subset \llbracket K^{\prime} \rrbracket$.

( " $\Leftarrow^{\prime \prime}$ ) we will show that $\llbracket K \rrbracket \subset \llbracket K^{\prime} \rrbracket$ implies $K^{\prime} \subset K$. As both $\llbracket K \rrbracket$ and $\llbracket K^{\prime} \rrbracket$ are CISs they are by definition $T h$-dense. Therefore, from Corollary 3.1 we get $T h\left(\llbracket K^{\prime} \rrbracket\right) \subset T h(\llbracket K \rrbracket)$. From the bijection between theories and CISs, we get $T h(\llbracket K \rrbracket)=K$ and $T h\left(\llbracket K^{\prime} \rrbracket\right)=K^{\prime}$. Thus, $K^{\prime} \subset K$.

(2) From (1) we have that $K \subset K^{\prime}$ iff $\llbracket K^{\prime} \rrbracket \subset \llbracket K \rrbracket$. Let $K$ stand for $T h(A)$ and $K^{\prime}$ stand for $\operatorname{Th}(B)$. So,

$$
T h(A) \subset T h(B) \text { iff } \llbracket T h(B) \rrbracket \subset \llbracket T h(A) \rrbracket .
$$

From the bijection between CISs and theories, we have that $\llbracket T h(B) \rrbracket=B$ and $\llbracket T h(A) \rrbracket=A$. Therefore, $T h(A) \subseteq T h(B)$ iff $B \subseteq A$.

Observation 3.5. Given two theories $K$ and $K^{\prime}$, and a model $M$ :

(1) $M \models K \cup K^{\prime}$ iff $M \in \llbracket K \rrbracket \cap \llbracket K^{\prime} \rrbracket$; 
(2) $\llbracket K \cup K^{\prime} \rrbracket=\llbracket K \rrbracket \cap \llbracket K^{\prime} \rrbracket$;

(3) $\llbracket C n\left(K \cup K^{\prime}\right) \rrbracket=\llbracket K \rrbracket \cap \llbracket K^{\prime} \rrbracket$.

Proof. (1) ( " $\Rightarrow$ ") We will show that $M \models K \cup K^{\prime}$ implies $M \in \llbracket K \rrbracket \cap \llbracket K^{\prime} \rrbracket$. From $M \models K \cup K^{\prime}$, we get that $M \models K$ and $M \models K^{\prime}$. Therefore, $M \in \llbracket K \rrbracket$ and $M \in \llbracket K^{\prime} \rrbracket$ which means that $M \in \llbracket K \rrbracket \cap \llbracket K^{\prime} \rrbracket$.

(" $\Leftarrow^{\prime \prime}$ ) We will show that $M \in \llbracket K \rrbracket \cap \llbracket K^{\prime} \rrbracket$ implies $M \models K \cup K^{\prime}$. From $M \in \llbracket K \rrbracket$ and $M \in \llbracket K^{\prime} \rrbracket$ we get that $M \models K$ and $M \models K^{\prime}$. Therefore, $M \models K \cup K^{\prime}$.

(2) We need to show that $M \in \llbracket K \cup K^{\prime} \rrbracket$ iff $M \in \llbracket K \rrbracket \cap \llbracket K^{\prime} \rrbracket$;

From definition, $M \in \llbracket K \cup K^{\prime} \rrbracket$ iff $M \models K \cup K^{\prime}$ which from (1) above gives us:

$$
M \models K \cup K^{\prime} \text { iff } M \in \llbracket K \rrbracket \cap \llbracket K^{\prime} \rrbracket .
$$

Therefore, $M \in \llbracket K \cup K^{\prime} \rrbracket$ iff $M \in \llbracket K \rrbracket \cap \llbracket K^{\prime} \rrbracket$.

(3) We need to show that $M \in \llbracket C n\left(K \cup K^{\prime}\right) \rrbracket$ iff $\llbracket K \rrbracket \cap \llbracket K^{\prime} \rrbracket$.

As $C n$ is Tarskian, a formula $\varphi \in C n\left(K \cup K^{\prime}\right)$ iff $M \models \varphi$, for all $M \in \llbracket K \cup K^{\prime} \rrbracket$. This means that,

$$
M \models C n\left(K \cup K^{\prime}\right) \text { iff } M \in \llbracket K \cup K^{\prime} \rrbracket .
$$

Moreover, from definition we have that $M \in \llbracket C n\left(K \cup K^{\prime}\right) \rrbracket$ iff $M \models C n\left(K \cup K^{\prime}\right)$. Therefore,

$$
M \in \llbracket C n\left(K \cup K^{\prime}\right) \rrbracket \text { iff } M \in \llbracket K \cup K^{\prime} \rrbracket .
$$

This together with (2) above gives us that $M \in \llbracket C n\left(K \cup K^{\prime}\right) \rrbracket$ iff $M \in \llbracket K \rrbracket \cap \llbracket K^{\prime} \rrbracket$.

Proposition 3.5. Given two theories $K$ and $K^{\prime}$,

1. $T h\left(\llbracket K \rrbracket \cap \llbracket K^{\prime} \rrbracket\right)=C n\left(K \cup K^{\prime}\right)$;

2. $T h\left(\llbracket K \rrbracket \cup \llbracket K^{\prime} \rrbracket\right)=K \cap K^{\prime}$.

Proof. We will show items (1) and (2) separately.

(1) We will show that $T h\left(\llbracket K \rrbracket \cap \llbracket K^{\prime} \rrbracket\right)=C n\left(K \cup K^{\prime}\right)$.

From the statement (3) of Observation 3.5, we get $\llbracket K \rrbracket \cap \llbracket K^{\prime} \rrbracket=\llbracket C n\left(K \cup K^{\prime}\right) \rrbracket$. Thus, $T h\left(\llbracket K \rrbracket \cap \llbracket K^{\prime} \rrbracket\right)=T h\left(\llbracket C n\left(K \cup K^{\prime}\right) \rrbracket\right)$.

Moreover, from the bijection between CISs and theories, we have that

$$
T h\left(\llbracket C n\left(K \cup K^{\prime}\right) \rrbracket\right)=C n\left(K \cup K^{\prime}\right) .
$$

Therefore,

$$
T h\left(\llbracket K \rrbracket \cap \llbracket K^{\prime} \rrbracket\right)=C n\left(K \cup K^{\prime}\right)
$$


(2) We will show that (a) $K \cap K^{\prime} \subseteq T h\left(\llbracket K \rrbracket \cup \llbracket K^{\prime} \rrbracket\right)$ and (b) $T h\left(\llbracket K \rrbracket \cup \llbracket K^{\prime} \rrbracket\right) \subseteq K \cap K^{\prime}$.

(a) $K \cap K^{\prime} \subseteq T h\left(\llbracket K \rrbracket \cup \llbracket K^{\prime} \rrbracket\right)$. This proof explore the reverse-monotonicity of the functions $T h$ and $\mathbb{\llbracket}$, as well as the bijection between CISs and theories. As $\llbracket \rrbracket$ is reverse-monotonic and $K \cap K^{\prime}$ are subsets of both $K$ and $K^{\prime}$, we get that $\llbracket K \rrbracket \subseteq \llbracket K \cap K^{\prime} \rrbracket$ and $\llbracket K^{\prime} \rrbracket \subseteq \llbracket K \cap K^{\prime} \rrbracket$. Therefore, $\llbracket K \rrbracket \cup \llbracket K^{\prime} \rrbracket \subseteq \llbracket K \cap K^{\prime} \rrbracket$. This implies, from the reverse-monotonicity of $T h$, that

$$
T h\left(\llbracket K \cap K^{\prime} \rrbracket\right) \subseteq T h\left(\llbracket K \rrbracket \cup \llbracket K^{\prime} \rrbracket\right) .
$$

From the bijection between CISs and theories, we have that,

$$
T h\left(\llbracket K \cap K^{\prime} \rrbracket\right)=K \cap K^{\prime} .
$$

Therefore, $K \cap K^{\prime} \subseteq T h\left(\llbracket K \rrbracket \cup \llbracket K^{\prime} \rrbracket\right)$.

(b) $T h\left(\llbracket K \rrbracket \cup \llbracket K^{\prime} \rrbracket\right) \subseteq K \cap K^{\prime}$. Let us suppose for contradiction that $T h\left(\llbracket K \rrbracket \cup \llbracket K^{\prime} \rrbracket\right) \nsubseteq \subseteq K \cap K^{\prime}$. Thus, there is a formula $\alpha \in T h\left(\llbracket K \rrbracket \cup \llbracket K^{\prime} \rrbracket\right)$ such that $\alpha \notin K \cap K^{\prime}$. This means that either $\alpha \notin K$ or $\alpha \notin K^{\prime}$.

From definition of $T h$, we have

$$
\varphi \in T h\left(\llbracket K \rrbracket \cup \llbracket K^{\prime} \rrbracket\right) \text { iff } M \models \varphi \text {, for all } M \in \llbracket K \rrbracket \cup \llbracket K^{\prime} \rrbracket \text {. }
$$

As $\alpha \in T h\left(\llbracket K \rrbracket \cup \llbracket K^{\prime} \rrbracket\right)$, we get

$$
M \models \alpha \text {, for all } M \in \llbracket K \rrbracket \cup \llbracket K^{\prime} \rrbracket .
$$

Recall that

$$
\varphi \in K \text { iff } M=\varphi \text {, for all } M \in \llbracket K \rrbracket \text {. }
$$

As $\alpha \notin K$ or $\alpha \notin K^{\prime}$, we get from the expression above that either there is a model $M \in \llbracket K \rrbracket$ such that $M \not \models \alpha$, or there is a models $M \in \llbracket K^{\prime} \rrbracket$ such that $M \not \models \alpha$. In either case, we get that there is a models $M \in \llbracket K \rrbracket \cup \llbracket K^{\prime} \rrbracket$ such that $M \not \forall \alpha$ which contradicts (A.4). So, we conclude that $T h\left(\llbracket K \rrbracket \cup \llbracket K^{\prime} \rrbracket\right) \subseteq K \cap K^{\prime}$.

From (a) and (b), we conclude that $T h\left(\llbracket K \rrbracket \cup \llbracket K^{\prime} \rrbracket\right)=K \cap K^{\prime}$.

Corollary 3.3. $\llbracket \cap X \rrbracket=\bigcup_{Y \in X} \llbracket Y \rrbracket$, for all sets of theories $X$.

Proof. From Proposition 3.5, $K \cap K^{\prime}=T h\left(\llbracket K \rrbracket \cup \llbracket K^{\prime} \rrbracket\right)$. This implies

$$
\llbracket K \cap K^{\prime} \rrbracket=\llbracket T h\left(\llbracket K \rrbracket \cup \llbracket K^{\prime} \rrbracket\right) \rrbracket .
$$


Now, we need the bijection $\tau$ from Theorem 3.2 which is a map between theories and CISs. Given a CIS $Y$, this bijection implies that $\llbracket T h(Y) \rrbracket=Y$. Making $Y=\llbracket K \rrbracket \cup \llbracket K^{\prime} \rrbracket$ leads to

$$
\llbracket T h\left(\llbracket K \rrbracket \cup \llbracket K^{\prime} \rrbracket\right) \rrbracket=\llbracket K \rrbracket \cup \llbracket K^{\prime} \rrbracket .
$$

Therefore, this together with (A.5) implies

$$
\llbracket K \cap K^{\prime} \rrbracket=\llbracket K \rrbracket \cup \llbracket K^{\prime} \rrbracket
$$

Now, instead of considering the intersection of only two theories, we take the intersection of a nonempty set of theories $X$. Therefore, we have that $\llbracket \cap X \rrbracket=\llbracket Y_{1} \rrbracket \cup \llbracket Y_{2} \rrbracket \cup \cdots \cup \llbracket Y_{n} \rrbracket \cup \ldots$, where each $Y_{i}$ is a theory of $X$. In other words, $\llbracket \cap X \rrbracket=\bigcup_{Y_{i} \in X} \llbracket Y_{i} \rrbracket$.

Proposition 3.6. If a non-tautological formula $\varphi$ belongs to a theory $K$, then $C n(\emptyset) \subset K \cap C n(\varphi)=$ $C n(\varphi)$, and either $\varphi$ is consistent or $K$ is inconsistent.

Proof. Let $K$ be a theory and $\varphi$ a non-tautological formula, that is, $\varphi \notin C n(\emptyset)$ and that $\varphi \in K$. As the logic is Tarskian and $\varphi \in K$, we have that $C n(\varphi) \subset K$ which implies that, $C n(\varphi) \cap K=C n(\varphi)$. Moreover, as $\varphi$ is not a tautology, we have $C n(\emptyset) \subset C n(\varphi)$. Thus, $C n(\emptyset) \subset K \cap C n(\varphi)=C n(\varphi)$.

If $\varphi$ is not consistent, that is, $\varphi \equiv \perp$ then $\perp \in K$. Therefore, $\mathrm{K}$ is inconsistent or $\varphi$ is consistent.

Theorem 3.3. Every ECF satisfies $\left(K_{1}^{-}\right)$to $\left(K_{6}^{-}\right)$.

Proof. Let ${ }_{\delta}$ be a ECF( ECFs were introduced in Definition 3.2). Moreover, let $K$ be a theory and $\varphi$ a formula:

( $\left.\mathbf{K}_{1}^{-}\right)$There are two cases to consider: $K{ }_{-} \varphi=K$ or $K{ }_{\delta} \varphi=K \cap \bigcap \delta(\varphi)$. As the intersections of theories is also a theory (remember that all sets in $\delta(\varphi)$ are theories), in both cases $K-{ }_{\delta} \varphi$ is a theory. Therefore, it satisfy $\left(\mathrm{K}_{1}^{-}\right)$.

$\left(\mathbf{K}_{2}^{-}\right)$For the case in which $K-\delta \varphi=K$ it is trivial that it satisfies $\left(\mathrm{K}_{2}^{-}\right)$. For the another case, that is, $K{ }_{-\delta} \varphi=K \cap \bigcap \delta(\varphi)$; as $K \cap \bigcap \delta(\varphi) \subseteq K$, we have that $K-{ }_{\delta} \varphi \subseteq K$.

$\left(\mathbf{K}_{3}^{-}\right)$If $\varphi \notin K$, then $C n(\varphi) \nsubseteq K$ which implies that $K \cap C n(\varphi) \neq C n(\varphi)$. Then, we have from definition of ECF that $K-{ }_{\delta} \varphi=K$.

( $\left.\mathbf{K}_{4}^{-}\right)$Let us suppose $\varphi \notin C n(\emptyset)$. Then, we have two cases to take into account: $\varphi \notin K$ and $\varphi \in K$. For the former, we have from $\left(\mathrm{K}_{3}^{-}\right)$that $K{ }_{\delta} \varphi=K$ which implies $\varphi \notin K{ }_{\delta} \varphi$. For the another case in which $\varphi \in K$, it follows from Proposition 3.6 that $C n(\emptyset) \subset K \cap \bigcap(\varphi)=C n(\varphi)$, and either $\varphi$ is consistent (i.e. $\neg \varphi \notin C n(\emptyset)$ ) or $\mathrm{K}$ is inconsistent (i.e. $\perp \in K$ ). Therefore, we have from definition of ECF that $K{ }_{\delta} \varphi=K \cap \bigcap \delta(\varphi)$. As, from condition (1) of CF, $\delta(\varphi)$ has at least one theory and from condition (2), since $\varphi \notin C n(\emptyset)$, its theories do not imply $\varphi$, we have that $\varphi \notin \bigcap \delta(\varphi)$. Hence, $\varphi \notin K \cap \bigcap \delta(\varphi)$, that is, $\varphi \notin K-\delta \varphi$.

$\left(\mathbf{K}_{5}^{-}\right)$We need to show that $K \subseteq K \dot{-} \varphi+\varphi$. The case that $K \dot{-} \varphi=K$ is trivial, so we proceed to the case $K \dot{-} \varphi=K \cap \delta(\varphi)$. This means that $\varphi$ is not a tautology and is in $K$. So, $\llbracket K \rrbracket \subseteq \llbracket \varphi \rrbracket$ and $\delta(\varphi) \subseteq \bar{\omega}(\varphi)$. This implies that $\neg \varphi \in \bigcap \delta(\varphi)$. Therefore, $\llbracket \cap \delta(\varphi) \rrbracket \subseteq \llbracket \neg \varphi \rrbracket$ which implies 
that $\llbracket K \rrbracket \cap \llbracket \cap \delta(\varphi) \rrbracket=\emptyset$. We then resort to the bijection between CISs and theories. We get $\llbracket K \dot{-} \varphi+\varphi \rrbracket=\llbracket K \dot{-} \varphi \rrbracket \cap \llbracket \varphi \rrbracket$, and $\llbracket K \dot{-} \varphi \rrbracket=\llbracket K \rrbracket \cup \llbracket \cap \varphi \rrbracket$. Substitution of the second in the first one gives us

$$
\llbracket K \dot{-} \varphi+\varphi \rrbracket=(\llbracket K \dot{-} \varphi \rrbracket \cap \llbracket \varphi \rrbracket) \cup(\llbracket \bigcap \delta(\varphi) \rrbracket \cap \llbracket \varphi \rrbracket) .
$$

As $\llbracket K \rrbracket \subseteq \llbracket \varphi \rrbracket$, we have that $\llbracket K \rrbracket \cap \llbracket \varphi \rrbracket=\llbracket K \rrbracket$. Moreover, $\llbracket \cap \delta(\varphi) \rrbracket$ has no models from $\varphi$ which means that $\llbracket \cap \delta(\varphi) \rrbracket \cap \llbracket \varphi \rrbracket=\emptyset$. Therefore, $\llbracket K \dot{-} \varphi+\varphi \rrbracket=\llbracket K \rrbracket$ which means that $K \dot{-} \varphi=K$. Thus $K \subseteq K \dot{-} \varphi+\varphi$.

$\left(\mathbf{K}_{6}^{-}\right)$Let $\varphi$ and $\psi$ be two formulae such that $C n(\varphi)=C n(\psi)$.If $\varphi, \psi \notin K$ then from $\left(\mathrm{K}_{3}^{-}\right)$, $K{ }_{\delta} \varphi=K{ }_{\delta} \psi=K$. Similarly, if $\varphi, \psi \in C n(\emptyset)$, then $K \cap C n(\varphi)=K \cap C n(\psi)=C n(\emptyset)$ which implies from definition that $K{ }_{-} \varphi=K$. In both cases $K{ }_{-} \varphi=K{ }_{\delta} \psi=K$.

If $\varphi \in K$ and $\varphi \notin C n(\emptyset)$, then from Proposition 3.6 we have that $C n(\emptyset) \subset K \cap C n(\varphi)=C n(\varphi)$, and either $\neg \varphi \notin C n(\emptyset)$ or $\perp \in K$. Therefore, we have from definition that $K{ }_{\delta} \varphi=K \cap \bigcap \delta(\varphi)$. Analogously, $K{ }_{-} \psi=K \cap \bigcap \delta(\psi)$. As $C n(\varphi)=C n(\psi)$ and $\delta$ is a CF, it follows from condition (3) of CF definition that $\delta(\varphi)=\delta(\psi)$. Thus, $K \cap \bigcap \delta(\varphi)=K \cap \bigcap \delta(\psi)$ which means that $K-\delta \varphi=$ $K-{ }_{\delta} \psi$.

Lemma 3.1. Let $K$ and $K^{\prime}$ be two theories such that $K^{\prime} \subseteq K$ and $\varphi$ a formula that it is in $K$ but not in $K^{\prime}$. If $K=K^{\prime}+\varphi$ then $\left(\llbracket K^{\prime} \rrbracket \backslash \llbracket K \rrbracket\right) \subseteq \llbracket \neg \varphi \rrbracket$.

Proof. Let us suppose for contradiction that $\llbracket K^{\prime} \rrbracket \backslash \llbracket K \rrbracket \nsubseteq \llbracket \neg \varphi \rrbracket$. For readability purpose, let us make $Z=\llbracket K^{\prime} \rrbracket \backslash \llbracket K \rrbracket$. Thus,

$$
Z \cap \llbracket K \rrbracket=\emptyset .
$$

As $Z \nsubseteq \llbracket \neg \varphi \rrbracket$, we get that there is some model from $\llbracket \varphi \rrbracket$ in $Z$, that is,

$$
Z \cap \llbracket \varphi \rrbracket \neq \emptyset .
$$

From hypothesis $K^{\prime} \subseteq K$, which implies from reverse-monotonicity of $\llbracket \rrbracket$ that $\llbracket K \rrbracket \subseteq \llbracket K^{\prime} \rrbracket$. Therefore,

$$
\llbracket K^{\prime} \rrbracket=\llbracket K \rrbracket \cup Z .
$$

So, we have

$$
\llbracket K^{\prime} \rrbracket \cap \llbracket \varphi \rrbracket=(\llbracket K \rrbracket \cap \llbracket \varphi \rrbracket) \cup(Z \cap \llbracket \varphi \rrbracket) .
$$

From hypothesis, $K=K^{\prime}+\varphi$. Therefore, $\llbracket K \rrbracket=\llbracket K^{\prime} \rrbracket \cap \llbracket \varphi \rrbracket$, which together with the expression above gives us:

$$
\llbracket K \rrbracket=(\llbracket K \rrbracket \cap \llbracket \varphi \rrbracket) \cup(Z \cap \llbracket \varphi \rrbracket) .
$$

From hypothesis $\varphi \in K$, which means that that $C n(\varphi) \subseteq K$ which implies from the reversemonotonicity of $\llbracket K \rrbracket$ that $\llbracket K \rrbracket \subseteq \llbracket \varphi \rrbracket$. Therefore, $\llbracket K \rrbracket \cap \llbracket \varphi \rrbracket=\llbracket K \rrbracket$. Substitution of this on the expression above render us:

$$
\llbracket K \rrbracket=\llbracket K \rrbracket \cup(Z \cap \llbracket \varphi \rrbracket) .
$$


This means that $(Z \cap \llbracket \varphi \rrbracket) \subseteq \llbracket K \rrbracket$. From (A.7) $Z \cap \llbracket \varphi \rrbracket \neq \emptyset$. Thus, we get that $Z \cap \llbracket K \rrbracket \neq \emptyset$ which contradicts (A.7).

Lemma A.2. Let $K$ be a theory, $Z$ be an interpretation set, and $Z^{+}=\llbracket T h(Z) \rrbracket$ be the CIS of the theory $T h(Z)$, then $T h(\llbracket K \rrbracket \cup Z)=T h\left(\llbracket K \rrbracket \cup Z^{+}\right)$.

Proof. Let $Z^{+}=\llbracket T h(Z) \rrbracket$, we will show (a) $T h\left(\llbracket K \rrbracket \cup Z^{+}\right) \subseteq T h(\llbracket K \rrbracket \cup Z)$ and (b) $T h(\llbracket K \rrbracket \cup Z) \subseteq$ $T h\left(\llbracket K \rrbracket \cup Z^{+}\right)$. Notice that as $Z^{+}$is a CIS, and both $Z$ and $Z^{+}$have the same theory, we get $Z \subseteq Z^{+}$.

(a) $T h\left(\llbracket K \rrbracket \cup Z^{+}\right) \subseteq T h(\llbracket K \rrbracket \cup Z)$. It is straightforward from the reverse-monotonicity of $T h$.

(b) Let us suppose for contradiction that $T h(\llbracket K \rrbracket \cup Z) \nsubseteq T h\left(\llbracket K \rrbracket \cup Z^{+}\right)$. Therefore, there is a formula $\alpha \in T h(\llbracket K \rrbracket \cup Z)$ such that $\alpha \notin T h\left(\llbracket K \rrbracket \cup Z^{+}\right)$.

From the reverse monotonicity of $T h$, we get that $T h(\llbracket K \rrbracket \cup Z) \subseteq T h(Z)$. This implies that

$$
\alpha \in T h(Z) \text {. }
$$

From the definition of $T h$, we have:

$$
\alpha \in T h(Y) \text { iff } M \models \alpha \text {, for all } M \in Y,
$$

where $Y$ is an interpretation set.

Moreover, as $\alpha \notin T h\left(\llbracket K \rrbracket \cup Z^{+}\right)$, we get that there is a model $M \in \llbracket K \rrbracket \cup Z^{+}$such that $M \not \models \alpha$. This means that, either $M \in \llbracket K \rrbracket$ or $M \in Z^{+}$.

- $M \in \llbracket K \rrbracket$. As $\alpha \in T h(\llbracket K \rrbracket \cup Z)$, we have that $M^{\prime} \models \alpha$, for all $M^{\prime} \in \llbracket K \rrbracket \cup Z$. This implies that $M \models \alpha$, which is a contradiction.

- $M \in Z^{+}$. This means that $\alpha \notin T h\left(Z^{+}\right)$. However, as $T h(Z)=T H\left(Z^{+}\right)$, it follows that $\alpha \notin T h(Z)$ which contradicts (A.8).

Observation 3.6. Given an AGM rational function $\dot{-}$, a theory $K$ and a non-tautological formula $\varphi \in K$. Moreover, let $Z=\llbracket K \dot{-} \varphi \rrbracket \backslash \llbracket K \rrbracket$. The following are true,

1. $K \dot{-} \varphi=K \cap T h(Z)$ and

2. $\neg \varphi \in T h(Z)$.

Proof. As $\dot{-}$ is AGM rational we have that $K \dot{-} \varphi \subseteq K$. Therefore, as the function $\llbracket \rrbracket$ is reversemonotonic it follows that $\llbracket K \rrbracket \subseteq \llbracket K \dot{-} \varphi \rrbracket$. As $Z=\llbracket K \dot{-} \varphi \rrbracket \backslash \llbracket K \rrbracket$, we get $\llbracket K \dot{-} \varphi \rrbracket=\llbracket K \rrbracket \cup Z$.

1. Let $Z^{+}=\llbracket T h(Z) \rrbracket$ be the CIS of the theory $T h(Z)$. So, we have that

$$
T h(Z)=T h\left(Z^{+}\right) .
$$


From $\llbracket K \dot{-} \varphi \rrbracket=\llbracket K \rrbracket \cup Z$ we get $K \dot{-} \varphi=T h(\llbracket K \rrbracket \cup Z)$. This implies from Lemma A.2 that

$$
K \dot{-} \varphi=T h\left(\llbracket K \rrbracket \cup Z^{+}\right) .
$$

From Lemma 3.5 we get

$$
K \dot{-} \varphi=K \cap T h\left(Z^{+}\right)
$$

As $T h(Z)=T h\left(Z^{+}\right)$, it follows that

$$
K \dot{-} \varphi=K \cap T h(Z)
$$

2. We will show that $\neg \varphi \in T h(Z)$. As $\dot{-}$ is AGM rational, we have, from the success and inclusion postulates, that $K \dot{-} \varphi \subset K$ and $\varphi \notin K$. This implies from the recovery postulate that $K \dot{-} \varphi+\varphi=K$. So, we have from Lemma 3.1 that $Z \subseteq \llbracket \neg \varphi \rrbracket$. This means that $\neg \varphi \in T h(Z)$.

Lemma 3.2. Let $K$ be a consistent theory, then $K=\bigcap \operatorname{dec}(K)$

Proof. We have to proof: (i) $K \subseteq \bigcap \operatorname{dec}(K)$ and (ii) $\bigcap \operatorname{dec}(K) \subseteq K$.

(i) $K \subseteq \bigcap \operatorname{dec}(K)$. From definition of $\operatorname{dec}$, a complete consistent theory $S$ is in $\operatorname{dec}(K)$ iff $K \subseteq S$. Thus, $K \subseteq \bigcap \operatorname{dec}(K)$.

(ii) $\bigcap \operatorname{dec}(K) \subseteq K$. Recall that

$$
\alpha \in K \text { iff } M \mid=\alpha \text {, for all } M \in \llbracket K \rrbracket \text {. }
$$

Let us suppose for contradiction that $\bigcap \operatorname{dec}(K) \nsubseteq K$. Thus, there is some formula $\alpha \in \bigcap \operatorname{dec}(K)$ such that $\alpha \notin K$. This gives us from the expression above that there is some model $M \in \llbracket K \rrbracket$ such that $M \not \models \alpha$. It implies from $M \in \llbracket K \rrbracket$ that $M \models K$ which means that $K \subseteq T h(M)$. This implies that

$$
T h(M) \in \operatorname{dec}(K) .
$$

Moreover, as $M \not \models \alpha$, we get

$$
\alpha \notin T h(M) .
$$

As by hypothesis $\alpha \in \bigcap \operatorname{dec}(K)$, we get that $\alpha \in S$, for all $S \in \operatorname{dec}(K)$. Therefore, as $T h(M) \in$ $\operatorname{dec}(K)$, we get $\alpha \in T h(M)$ which is a contradiction.

Proposition 3.7. Let - be a function that satisfies $\left(K_{1}^{-}\right)$to $\left(K_{6}^{-}\right)$. Then, $K-\varphi \neq K$ iff $C n(\emptyset) \subset$ $K \cap C n(\varphi)=C n(\varphi)$, and either $\neg \varphi \notin C n(\emptyset)$ or $\perp \in K$.

Proof. Let - be a contraction function satisfying all six basic postulates. 
' $\Rightarrow$ ': if $K-\varphi \neq K$, then $C n(\emptyset) \subset K \cap C n(\varphi)=C n(\varphi)$, and either $\neg \varphi \notin C n(\emptyset)$ or $\perp \in K$. By the contrapositive, if (i) $C n(\emptyset) \not \subset K \cap C n(\varphi)$, or (ii) $K \cap C n(\varphi) \neq C n(\varphi)$, or (iii) $\neg \varphi \in C n(\emptyset)$ and $\perp \notin K$, then $K-\varphi=K$. So we have to show that (i), (ii) and (iii) implies that $K-\varphi=K$.

(i) Let us suppose that $C n(\emptyset) \not \subset K \cap C n(\varphi)$. As $C n(\emptyset)$ is the set of all tautologies and it is present in every theory, it follows that $C n(\emptyset) \subseteq K \cap C n(\varphi)$ which together with $C n(\emptyset) \not \subset K \cap C n(\varphi)$ implies that

$$
K \cap C n(\varphi)=C n(\emptyset)
$$

We have now two cases to consider w.r.t $\varphi$ : (a) $\varphi \notin C n(\emptyset)$ or (b) $\varphi \in C n(\emptyset)$.

(a) $\varphi \notin C n(\emptyset)$, then $\varphi \notin K$, as $K \cap C n(\varphi)=C n(\emptyset)$. Thus, from $\left(\mathrm{K}_{3}^{-}\right), K-\varphi=K$.

(b) Suppose that $\varphi \in C n(\emptyset)$, that is $\varphi$ is a tautology, then $C n(\varphi)=C n(\emptyset)$. To show that $K-\varphi=K$, let us suppose for contradiction that

$$
K-\varphi \neq K
$$

As $C n(\varphi)=C n(\emptyset)$ and $C n(\emptyset)$ is present in every theory, we have that $C n(\varphi) \subseteq K-\varphi$. Therefore,

$$
\begin{aligned}
(K-\varphi) \cup C n(\varphi) & =K-\varphi \\
C n((K-\varphi) \cup C n(\varphi)) & =C n(K-\varphi) .
\end{aligned}
$$

Then, as $K-\varphi$ is a theory, it follows from idempotence that $C n(K-\varphi)=K-\varphi$. Thus from (A.9):

$$
C n((K-\varphi) \cup C n(\varphi))=K-\varphi \text {. }
$$

From definition of ' + ',

$$
(K-\varphi)+\varphi=C n((K-\varphi) \cup C n(\varphi)) .
$$

This together with (A.10) implies that

$$
(K-\varphi)+\varphi=K-\varphi
$$

As we have assumed that - satisfies all the six basic AGM postulates, we have from $\left(\mathrm{K}_{5}^{-}\right)$that $K \subseteq(K-\varphi)+\varphi$. Notice that this implies,

$$
(K-\varphi)+\varphi \not \subset K .
$$

From $\left(\mathrm{K}_{2}^{-}\right), K-\varphi \subseteq K$. Thus, as we have assumed $K-\varphi \neq K$, we have that $K-\varphi \subset K$ which together with (A.11) implies that $(K-\varphi)+\varphi \subset K$ which contradicts (A.12). So, we conclude $K-\varphi=K$.

(ii) Let us suppose that $K \cap C n(\varphi) \neq C n(\varphi)$. So, $\varphi \notin K$, which implies from $\left(\mathrm{K}_{3}^{-}\right)$that $K-\varphi=K$. 
(iii) Let us suppose $\neg \varphi \in C n(\emptyset)$ and $\perp \notin K$. Notice that $\neg \varphi \in C n(\emptyset)$ means $\varphi \equiv \perp$. Thus, as $\perp \notin K$, we have that $\varphi \notin K$. This implies, from $\left(\mathrm{K}_{3}^{-}\right)$, that $K-\varphi=K$.

Thus, we have from (i), (ii) and (iii) that $K-\varphi=K$. This conclude the proof for " $\Rightarrow$ ".

' $\Leftarrow$ ': Let us suppose that $C n(\emptyset) \subset K \cap C n(\varphi)=C n(\varphi)$ and either $\neg \varphi \notin C n(\emptyset)$ or $\perp \in K$.

As $K \cap C n(\varphi)=C n(\varphi)$, we have that $C n(\varphi) \subseteq K$, which means

$$
\varphi \in K \text {. }
$$

Moreover, from $C n(\emptyset) \subset K \cap C n(\varphi)=C n(\varphi)$ we have $C n(\emptyset) \subset C n(\varphi)$ which means $\varphi \notin C n(\emptyset)$. So, from $\left(\mathrm{K}_{4}^{-}\right), \varphi \notin K-\varphi$. Thus, $K-\varphi \neq K$, since $\varphi \in K$ and $\varphi \notin K-\varphi$.

Proposition 3.8. Let - be an $A G M$ rational contraction function, and $K$ a theory. Moreover, let $K^{\prime} \rrbracket K$ stand for $\llbracket K^{\prime} \rrbracket \backslash \llbracket K \rrbracket$. We construct the following function

$$
\delta(\varphi)=\left\{\begin{array}{cl}
T_{L} & \text { if } \varphi \in C n(\emptyset) \\
\operatorname{dec}(T h(K \dot{-} \varphi \rrbracket K) & \text { if } K \dot{-} \varphi \neq K \\
\bar{\omega}(\varphi) & \text { otherwise. }
\end{array}\right.
$$

The following are true,

1. $\delta$ is a choice function, and

2. if $\varphi \in K$ and $\varphi$ is not a tautology, then $K \dot{-} \varphi=K \cap \bigcap \delta(\varphi)$.

Proof. We will prove each item separately. For convenience let $Z=\llbracket K \dot{-} \varphi \rrbracket \backslash \llbracket K \rrbracket$.

1. We need to show that $\delta$ satisfies all three conditions for a choice function definition. Condition (3) which states that a choice function cannot be syntax sensitive is straightforward, as an AGM rational function is also not syntax-sensitive. We focus only on (1) and (2).

(1) We will show that $\delta(\varphi) \neq \emptyset$. We split it into two cases: either $\varphi$ is a tautology or it is not. In the case of being a tautology, $\delta(\varphi)=T_{L}$ which is not empty. For not being tautological, we get that $\delta(\varphi)$ results in $\bar{\omega}(\varphi)$ or $\operatorname{dec}(\operatorname{Th}(K \dot{-} \varphi \| K))$. The former is trivial, since $\bar{\omega}(\varphi)$ is not empty for non-tautological formulae (as the logic is closed under classical negation). For $\delta(\varphi)=\operatorname{dec}(T h(Z))$, we get from Observation 3.6 that $\neg \varphi \in T h(Z)$ which means that $\operatorname{dec}(T h(Z))$ is not empty.

(2) Let us suppose that $\varphi$ is not a tautology. We will show that $\delta(\varphi) \subseteq \bar{\omega}(\varphi)$. As $\varphi$ is not a tautology, we have from $\delta$ definition that $\delta(\varphi)$ is either $\bar{\omega}(\varphi)$ or $\operatorname{dec}(\operatorname{Th}(Z))$. Thus, we need to deal only with the latter case. From Observation 3.6 we get that $\neg \varphi \in T h(Z)$. This implies that every complete consistent theory in $\operatorname{dec}(\operatorname{Th}(Z))$ has $\neg \varphi$. Therefore, $\operatorname{dec}(\operatorname{Th}(Z)) \subseteq \bar{\omega}(\varphi)$, that is, $\operatorname{dec}(\varphi) \subseteq \bar{\omega}(\varphi)$. 
2. Let us suppose that $\varphi \in K$ and that $\varphi$ is not a tautology. $K \dot{-} \varphi=K \cap \bigcap \delta(\varphi)$. From Propositions 3.7 and 3.6 we have $K \dot{-} \varphi \neq K$. This implies, from the definition of $\delta$ that

$$
\delta(\varphi)=\operatorname{dec}(\operatorname{Th}(Z))
$$

Moreover, from Observation 3.6 we get that

$$
K \dot{-} \varphi=K \cap T h(Z)
$$

From Lemma 3.2, we have that $T h(Z)=\bigcap \operatorname{dec}(T h(Z))$. Therefore,

$$
K \dot{-} \varphi=K \cap \bigcap \operatorname{dec}(T h(Z))
$$

Thus, as $\delta(\varphi)=\operatorname{dec}(T h(Z))$, we get

$$
K \dot{-} \varphi=K \cap \bigcap \delta(\varphi)
$$

Theorem 3.5. Every BCF satisfy $\left(K_{1}^{-}\right)$to $\left(K_{7}^{-}\right)$.

Proof. Let $\dot{-}$ be a BCF w.r.t an annulment $\mu<$. So, $\dot{-}$ satisfies $\left(\mathrm{K}_{1}^{-}\right)$to $\left(\mathrm{K}_{6}^{-}\right)$, as from Corollary 3.5 it is a ECF. Moreover, let $K$ be a belief set, and $\varphi$ and $\psi$ two formulae. There is nothing to prove to the case that $K-\varphi \wedge \psi=K$, as it is trivial. Notice that this case also includes the possibility of both $\psi$ and $\varphi$ be tautologies. So we proceed to the case that one of them is not a tautology. Without loss of generality, let us fix $\psi$ as not being a tautology.

- Let us suppose that $C n(\varphi)=C n(\emptyset)$. So, $K \dot{-} \varphi=K$ and $\varphi \wedge \psi \equiv \psi$ which implies that

$$
K \dot{-} \varphi \cap K \dot{-} \psi=K \cap K \dot{-} \psi
$$

As from $\left(\mathrm{K}_{2}^{-}\right) K \dot{-} \psi \subseteq K$, we have that $K \cap(K \dot{-} \psi)=K \dot{-} \psi$. Thus, from (A.13)

$$
K \dot{-} \varphi \cap K \dot{-} \psi=K \dot{-} \psi
$$

Moreover, as $\varphi \wedge \psi \equiv \psi$, we from $\left(\mathrm{K}_{6}^{-}\right)$that $K \dot{-} \varphi \wedge \psi=K \dot{-} \psi$ which implies from (A.14) that

$$
K \dot{-} \varphi \cap K \dot{-} \psi=K \dot{-} \varphi \wedge \psi
$$

Thus, $K \dot{-} \varphi \cap K \dot{-} \psi \subseteq K \dot{-} \varphi \wedge \psi$.

- Let us suppose that $C n(\varphi) \neq C n(\emptyset)$. As $\max _{<}(\bar{\omega}(\varphi)) \subseteq \bar{\omega}(\varphi)$, and $\max _{<}(\psi) \subseteq \bar{\omega}(\psi)$, we have that $\max _{<}(\varphi) \cup \max _{<}(\psi) \subset \max _{<}(\bar{\omega}(\varphi)) \cup \max _{<}(\bar{\omega}(\psi))$. It well known that in a binary relation, $\max _{<}(A \cup B) \subseteq \max _{<}(A) \cup \max _{<}(B)$. Thus, $\max _{<}(\bar{\omega}(\varphi) \cup \bar{\omega}(\psi)) \subseteq \max _{<}(\bar{\omega}(\varphi)) \cup$ $\max _{<}(\bar{\omega}(\psi))$. As $\bar{\omega}(\varphi \wedge \psi)=\bar{\omega}(\varphi) \cup \bar{\omega}(\psi)$, we have that $\max _{<}(\bar{\omega}(\varphi \wedge \psi)) \subseteq \max _{<}(\bar{\omega}(\varphi)) \cup$ $\max _{<}(\bar{\omega}(\psi))$ which means that $\bar{\omega}(\varphi) \subseteq \bar{\omega}(\varphi \wedge \psi)$. This implies that 


$$
K-\varphi \wedge \psi=K \cap \bigcap \mu_{<}(\varphi \wedge \psi)
$$

Moreover,

$$
\begin{aligned}
K \dot{-} \varphi \cap K \dot{-} \psi & =\left(K \cap \bigcap \mu_{<}(\varphi)\right) \cap\left(K \cap \bigcap \mu_{<}(\psi)\right) \\
& =K \cap\left(\bigcap \mu_{<}(\varphi)\right) \cap\left(\bigcap \mu_{<}(\psi)\right) \\
& =K \cap \bigcap\left(\mu_{<}(\varphi) \cup \mu_{<}(\psi)\right) .
\end{aligned}
$$

Given two collection of sets $A$ and $B$, if $A \subseteq B$, then $\bigcap B \subseteq \bigcap A$. Thus, as $\mu_{<}(\varphi \wedge \psi) \subseteq$ $\mu_{<}(\varphi) \cup \mu_{<}(\psi)$, we have that $\bigcap \mu_{<}(\varphi) \cup \mu_{<}(\psi) \subseteq \bigcap \mu_{<}(\varphi \wedge \psi) \subseteq \mu_{<}(\varphi)$. This, together with (A.15) and (A.16), implies that

$$
\begin{gathered}
K \cap \bigcap\left(\mu_{<}(\varphi) \cup \mu_{<}(\psi)\right) \subseteq K \cap \bigcap \mu_{<}(\varphi \wedge \psi) \\
K \dot{-} \varphi \cap K \dot{-} \psi \subseteq K \dot{-} \varphi \wedge \psi .
\end{gathered}
$$

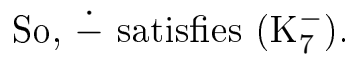

Lemma 3.4. Let $A$ and $B$ be two sets ordered by a contra-headed $<$ that satisfies mirroring. If some maximal element of $A$ is also a maximal element of $A \cup B$, then $\max _{<}(A) \subseteq \max _{<}(A \cup B)$.

Proof. Let us suppose, by contradiction, that there is a maximal element $S$ in $A$ that is also a maximal element in $A \cup B$, and $\max _{<}(A) \nsubseteq \max _{<}(A \cup B)$. So, there is a maximal element $S^{\prime}$ of $A$ that is not in $\max _{<}(A \cup B)$. Clearly, $S \neq S^{\prime}$, and $S \nless S^{\prime}$ and $S^{\prime} \notin S$, as both are maximal in $A \cup B$. As $\max _{<}(A)$ is not included in $\max _{<}(A \cup B)$, we have that there is a maximal element $S^{\prime \prime}$ of $B$ in $\max _{<}(A \cup B)$. Therefore, $S^{\prime}<S^{\prime \prime}$ which implies from condition 1 that $S<S^{\prime \prime}$ which is a contradiction since $S$ is maximal. Therefore, we conclude that $\max _{<}(A) \subseteq \max _{<}(A \cup B)$.

Lemma 3.5. Let $\mu_{<}$be an annulment. Every complete theory $S$ in $\mu_{<}(\varphi \wedge \psi)$ is either in $S \in \mu_{<}(\varphi)$ or $S \in \mu_{<}(\psi)$.

Proof. Let us suppose for contradiction that there is a complete theory $S$ in $\mu_{<}(\varphi \wedge \psi)$ such that $S \notin \mu_{<}(\varphi)$ and $S \notin \mu_{<}(\psi)$. This means that there is a $S^{\prime} \in \bar{\omega}(\varphi)$ such that $S<S^{\prime}$. As $S \in$ $\mu_{<}(\varphi \wedge \psi)=\max _{<}(\bar{\omega}(\varphi) \cup \bar{\omega}(\psi))$ and $S^{\prime} \in \bar{\omega}(\varphi)$, both $S$ and $S^{\prime}$ belongs to $\bar{\omega}(\varphi) \cup \bar{\omega}(\psi)$. Thus, due to $S<S^{\prime}$, we have $S \notin \max _{<}(\bar{\omega}(\varphi) \cup \bar{\omega}(\psi))$. Thus, $S \notin \mu_{<}(\varphi \wedge \psi)$ which is a contradiction. Therefore, we conclude that $S \in \mu_{<}(\varphi)$ or $S \in \mu_{<}(\psi)$.

Theorem 3.6. Let $\dot{-}$ be a $B C F$ w.r.t an annulment $\mu_{<}$. If $<$satisfies mirroring, then $\dot{-}$ satisfies $\left(K_{8}^{-}\right)$. 
Proof. Let us suppose that $\dot{-}$ is a BCF w.r.t an annulment $\mu_{<}$and that $<$satisfies mirroring. Moreover, let $K$ be a belief set and $\varphi$ a formula such that $\varphi \notin K \dot{-} \varphi \wedge \psi$ which means that $\varphi \wedge \psi \notin K \dot{-} \varphi \wedge \psi$ as well. Thus, from definition of BCF, it follows that

$$
\begin{aligned}
K \dot{-} \varphi \wedge \psi & =K \cap \bigcap \mu_{<}(\varphi \wedge \psi) \text { and } \\
K \dot{-} \varphi & =K \cap \bigcap \mu_{<}(\varphi) .
\end{aligned}
$$

As $\varphi$ is not in the contraction, then necessarily some $S$ in $\mu_{<}(\varphi \wedge \psi)$ is from $\bar{\omega}(\varphi)$. So, from Lemma 3.5, $S \in \mu_{<}(\varphi)$. Then, from Lemma 3.4, $\mu_{<}(\varphi) \subseteq \mu_{<}(\varphi \wedge \psi)$ which implies that $\left(\bigcap \mu_{<}(\varphi \wedge \psi)\right) \subseteq$ $\left(\bigcap \mu_{<}(\varphi)\right)$.

$$
K \cap \bigcap \mu_{<}(\varphi \wedge \psi) \subseteq K \cap \bigcap \mu_{<}(\varphi)
$$

which from (A.17) and (A.18) implies

$$
K \dot{-} \varphi \wedge \psi \subseteq K \dot{-} \varphi
$$

That is, $\dot{-}$ satisfies $\left(\mathrm{K}_{8}^{-}\right)$.

Observation A.2. Let $A, B$ and $C$ be three sets such that $C \cap B=\emptyset$. If $C \cup B \subseteq C \cup A$ then $B \subseteq A$.

Proof. Let $S$ be a theory in $B$, we have to show that $S \in A$. As $C \cap B=\emptyset$, we have that $S \notin C$. From $S \in B$, it follows that $S \in C \cup B$ which from hypothesis implies that $S \in C \cup A$. Therefore, as $S \notin C$, we get $S \in A$.

Lemma A.3. Given a theory $K$ and a formula $\varphi$ : $\operatorname{dec}(K+\varphi)=\operatorname{dec}(K) \cap \omega(\varphi)$.

Proof. First, from Lemma 3.2, $\operatorname{dec}(K+\varphi)=\operatorname{dec}(K) \cap \operatorname{dec}(\operatorname{Cn}(\varphi))$. Recall that $\omega$ each formula for the set of complete consistent theories that contains such a formula, whereas dec maps theories to the collection of all complete consistent theories that contains such theory. Therefore, $\omega(\varphi)=$ $\operatorname{dec}(C n(\varphi))$. This implies that

$$
\operatorname{dec}(K+\varphi)=\operatorname{dec}(K) \cap \omega(\varphi) .
$$

Observation 3.7. Let $K$ be a theory, and $\dot{-}_{\delta}$ be an $E C F$, there is a saturated $K$-grounded choice function $\delta^{\prime}$ such that $K \dot{-}_{\delta} \varphi=K \dot{-}_{\delta^{\prime}} \varphi$, for every formula $\varphi$.

Proof. Let $\dot{-}_{\delta}$ be an ECF. We will construct a $K$-grounded choice function $\delta^{\prime}$, such that $K \dot{-}_{\delta} \varphi=$ $K \dot{-}_{\delta^{\prime}} \varphi$. We make $\delta^{\prime}$ mimic $\delta$, whenever $\varphi \in K$, but $\varphi$ is not a tautology. Otherwise, if $\varphi$ is a tautology we make $\delta^{\prime}$ chooses the whose set of the complete theories; and in the case that $\varphi \notin K$, we fill up $\delta(\varphi)$ with $\bar{\omega}(\varphi)$. Thus, 


$$
\delta^{\prime}(\varphi)=\left\{\begin{array}{cl}
\operatorname{dec}(\bigcap \delta(\varphi)) & \text { if } \varphi \in K \text { and } C n(\varphi) \neq C n(\emptyset) \\
\operatorname{dec}(K) \cap \bar{\omega}(\varphi) & \text { if } \varphi \notin K \\
T_{L} & \text { if } C n(\varphi)=C n(\emptyset) .
\end{array}\right.
$$

It is trivial that $\delta^{\prime}$ is a choice function and K-grounded. We have to show that (i) $\delta^{\prime}$ is saturated and (ii) $K \dot{-}_{\delta} \varphi=K \dot{-}_{\delta^{\prime}} \varphi$.

(i) We need to show that $\delta^{\prime}(\varphi)=\operatorname{dec}\left(\bigcap \delta^{\prime}(\varphi)\right)$. Let $\varphi$ be a non-tautological formula in $K$, and $K^{\prime}$ be a theory. So, from Lemma $3.2, K^{\prime}=\bigcap \operatorname{dec}\left(K^{\prime}\right)$ which implies that

$$
\operatorname{dec}\left(K^{\prime}\right)=\operatorname{dec}\left(\bigcap \operatorname{dec}\left(K^{\prime}\right)\right)
$$

From $\delta^{\prime}$ construction: (a) $\delta^{\prime}(\varphi)=\operatorname{dec}(\bigcap \delta(\varphi))$ or $(\mathrm{b}) \delta^{\prime}(\varphi)=\operatorname{dec}(K) \cap \bar{\omega}(\varphi)$.

(a) $\delta^{\prime}(\varphi)=\operatorname{dec}(\bigcap \delta(\varphi))$. Let $K^{\prime}$ stand for $\bigcap \delta(\varphi)$. Thus, $\operatorname{dec}\left(K^{\prime}\right)=\operatorname{dec}(\bigcap \delta(\varphi))$ which means that

$$
\operatorname{dec}\left(K^{\prime}\right)=\delta^{\prime}(\varphi)
$$

Substitution of $\delta^{\prime}(\varphi)=\operatorname{dec}\left(K^{\prime}\right)$ in (A.19) gives us, $\delta^{\prime}(\varphi)=\operatorname{dec}\left(\bigcap \delta^{\prime}(\varphi)\right)$.

(b) $\delta^{\prime}(\varphi)=\operatorname{dec}(K) \cap \bar{\omega}(\varphi)$. From Lemma A.3, we have that

$$
\operatorname{dec}(K+\neg \varphi)=\operatorname{dec}(K) \cap \omega(\neg \varphi) .
$$

Simplifying, $\operatorname{dec}(K+\neg \varphi)=\operatorname{dec}(K) \cap \bar{\omega}(\varphi)$. This implies from Lemma 3.2 that $K+\neg \varphi=$ $\bigcap(\operatorname{dec}(K) \cap \bar{\omega}(\varphi))$. Therefore,

$$
K+\neg \varphi=\bigcap \delta^{\prime}(\varphi)
$$

Let $K^{\prime}$ stand for $K+\neg \varphi$. So, $\operatorname{dec}\left(K^{\prime}\right)=\operatorname{dec}(K+\neg \varphi)$ which from (A.20) implies

$$
\operatorname{dec}\left(K^{\prime}\right)=\delta^{\prime}(\varphi)
$$

Substitution of the expression above in (A.19) gives us

$$
\delta^{\prime}(\varphi)=\operatorname{dec}\left(\bigcap \delta^{\prime}(\varphi)\right)
$$

(ii) In the case that $\varphi$ is tautological or $\varphi \notin K$, we have from definition of ECF that $K \dot{-}_{\delta} \varphi=$ $K \dot{-}_{\delta^{\prime}}=K$. So we proceed to the case that $\varphi$ is not tautological and $\varphi \in K$. We have from definition of ECF that

$$
K \dot{-}_{\delta} \varphi=K \cap \bigcap \delta(\varphi) \quad \text { and } \quad K \dot{-}_{\delta^{\prime}} \varphi=K \cap \bigcap \delta^{\prime}(\varphi)
$$


From the construction of $\delta^{\prime}$ we have that $\delta^{\prime}(\varphi)=\operatorname{dec}(\bigcap \delta(\varphi))$. This means that

$$
\bigcap \delta^{\prime}(\varphi)=\bigcap \operatorname{dec}(\bigcap \delta(\varphi))
$$

As $K \dot{-}{ }_{\delta} \varphi=K \cap \bigcap \delta(\varphi)$, we get from the expression above

$$
\begin{aligned}
K \dot{-}_{\delta} \varphi & =K \cap \bigcap \operatorname{dec}(\bigcap \delta(\varphi) \\
& =K \cap \bigcap \delta^{\prime}(\varphi) .
\end{aligned}
$$

Therefore, $K \dot{-}_{\delta} \varphi-K \dot{-}_{\delta^{\prime}} \varphi$.

Lemma A.4. Given two theories $K$ and $K^{\prime}: \operatorname{dec}\left(K \cap K^{\prime}\right)=\operatorname{dec}(K) \cup \operatorname{dec}\left(K^{\prime}\right)$.

Proof. We need to show that $\operatorname{dec}(K) \cup \operatorname{dec}\left(K^{\prime}\right) \subseteq \operatorname{dec}\left(K \cap K^{\prime}\right)$ and $\operatorname{dec}\left(K \cap K^{\prime}\right) \subseteq \operatorname{dec}(K) \cup \operatorname{dec}\left(K^{\prime}\right)$. The former is straightforward from the reverse-monotonicity of $d e c$. So we proceed to the latter case: $\operatorname{dec}\left(K \cap K^{\prime}\right) \subseteq \operatorname{dec}(K) \cup \operatorname{dec}\left(K^{\prime}\right)$.

We will show that $\operatorname{dec}\left(K \cap K^{\prime}\right) \subseteq \operatorname{dec}(K) \cup \operatorname{dec}\left(K^{\prime}\right)$. Let $S \in \operatorname{dec}\left(K \cap K^{\prime}\right)$, we have to show that $S \in \operatorname{dec}(K) \cup \operatorname{dec}\left(K^{\prime}\right)$. As $S$ is consistent, there is a model $M$ such that $M \models S$. Thus, as $K \cap K^{\prime} \subseteq S$, we get that $M \models K \cap K^{\prime}$ which implies that $M \in \llbracket K \cap K^{\prime} \rrbracket$. From item (2) of Observation 3.5 we get that $M \in \llbracket K \cup K^{\prime} \rrbracket$. This means that $M \models K \cup K^{\prime}$. Therefore, $M \models K$ which implies that $M \in \operatorname{dec}(K)$. Therefore, $K \subseteq T h(M)$ which means that $T h(M) \in \operatorname{dec}(K) \cup \operatorname{dec}\left(K^{\prime}\right)$. As $T h(M)=S$, we get $S \in \operatorname{dec}(K) \cup \operatorname{dec}\left(K^{\prime}\right)$.

Observation A.3. Given three sets $A, B$ and $C$. If $A \cup B \subseteq A \cup C$ and $A \cap B=\emptyset$ and $A \cap C=\emptyset$ then $B \subseteq C$.

Proof. Let us suppose for contradiction that $A \cup B \subseteq A \cup C$ and $A \cap B=\emptyset$ and $A \cap C=\emptyset$, but $B \nsubseteq C$. Thus, there is some element $x \in B$ such that $X \notin C$. As $A \cup B \subseteq A \cup C$, this implies that $x \in A \cup C$. Therefore, $x \in A$ which is a contradiction since $A \cap B=\emptyset$.

Lemma 3.6. Let $\dot{-}_{\delta}$ be an $E C F$, and $\varphi$ and $\psi$ be two non-tautological formulae. If $\delta$ is a saturated $K$-grounded choice function, and $\varphi \wedge \psi \in K$ then

(C1) If $\dot{-}_{\delta}$ satisfies $\left(K_{7}^{-}\right)$, then $\delta(\varphi \wedge \psi) \subseteq \delta(\varphi) \cup \delta(\psi)$;

(C2) If $\dot{-}_{\delta}$ satisfies $\left(K_{8}^{-}\right)$, then $\delta(\varphi) \subseteq \delta(\varphi \wedge \psi)$ if there is some theory $S$ of $\delta(\varphi)$ in $\delta(\varphi \wedge \psi)$.

Proof. As $\varphi \wedge \psi$ is not a tautology and $\varphi \wedge \psi \in K$, we get that

$$
\begin{aligned}
K \dot{-}_{\delta} \varphi \wedge \psi & =K \cap \bigcap \delta(\varphi \wedge \psi) \\
K \dot{-}_{\delta} \varphi & =K \cap \bigcap \delta(\varphi) \\
K \dot{-}_{\delta} \psi & =K \cap \bigcap \delta(\psi) .
\end{aligned}
$$


(C1:) Let us suppose that $\dot{-}_{\delta}$ satisfies $\left(\mathrm{K}_{7}^{-}\right)$, we will show $\delta(\varphi \wedge \psi) \subseteq \delta(\varphi) \cup \delta(\psi)$. Then, $K \dot{-}_{\delta} \varphi \cap$ $K \dot{-}_{\delta} \psi \subseteq K \dot{-}_{\delta} \varphi \wedge \psi$ which implies that

$$
K \cap(\bigcap \delta(\varphi)) \cap(\bigcap \delta(\psi)) \subseteq K \cap \bigcap \delta(\varphi \wedge \psi)
$$

As dec is reverse monotonic, we get

$$
\operatorname{dec}(K \cap \bigcap \delta(\varphi \wedge \psi)) \subseteq \operatorname{dec}(K \cap(\bigcap \delta(\varphi)) \cap(\bigcap \delta(\psi)))
$$

From Lemma A.4, we get

$$
\operatorname{dec}(K) \cup \operatorname{dec}(\bigcap \delta(\varphi \wedge \psi)) \subseteq \operatorname{dec}(K) \cup \operatorname{dec}(\bigcap \delta(\varphi)) \cup \operatorname{dec}(\bigcap \delta(\psi))
$$

As $\delta$ is $K$-grounded we have that $\delta(\alpha)=\operatorname{dec}(\bigcap \delta(\alpha))$, for every non-tautological formula $\alpha \in K$. Therefore,

$$
\operatorname{dec}(K) \cup \delta(\varphi \wedge \psi) \subseteq \operatorname{dec}(K) \cup \delta(\varphi) \cup \delta(\psi)
$$

Moreover, as $\varphi \wedge \psi \in K$, we have that $\delta$ for either $\varphi, \psi$ and $\varphi \wedge \psi$ has no complete theory of $K$. Therefore, from Observation D.4,

$$
\delta(\varphi \wedge \psi) \subseteq \delta(\varphi) \cup \delta(\psi)
$$

(C2:) Let us suppose that $\dot{-}_{\delta}$ satisfies $\left(\mathrm{K}_{8}^{-}\right)$, and that there is some theory $S$ of $\delta(\varphi)$ in $\delta(\varphi \wedge \psi)$. We will show $\delta(\varphi) \subseteq \delta(\varphi \wedge \psi)$. From $\left(\mathrm{K}_{8}^{-}\right)$we have

$$
K \dot{-}_{\delta} \varphi \wedge \psi \subseteq K \dot{-}_{\delta} \varphi
$$

This means that

$$
K \cap \bigcap \delta(\varphi \wedge \psi) \subseteq K \cap \delta(\varphi)
$$

As dec is reverse-monotonic, we get

$$
K \cap \delta(\varphi) \subseteq K \cap \bigcap \delta(\varphi \wedge \psi)
$$

From Lemma A.4, we have

$$
\operatorname{dec}(K) \cup \operatorname{dec}(\bigcap \delta(\varphi)) \subseteq \operatorname{dec}(K) \cup \operatorname{dec}(\bigcap \delta(\varphi \wedge \psi))
$$

As $\delta$ is $K$-grounded, it follows that

$$
\operatorname{dec}(K) \cup \delta(\varphi) \subseteq \operatorname{dec}(K) \cup \delta(\varphi \wedge \psi) .
$$

Therefore, as $K$ does not share any complete consistent theory neither with $\varphi$ neither with 
$\varphi \wedge \psi$, it follows from Observation D.4

$$
\delta(\varphi) \subseteq \delta(\varphi \wedge \psi)
$$

Observation A.4. Given a theory $K$, an $E C F \dot{-}_{\delta}$ that satisfies $\left(K_{7}^{-}\right)$and $\left(K_{8}^{-}\right)$, and two nontautological formulae $\varphi$ and $\psi$. If $\dot{-}_{\delta}$ is $K$-grounded, and both $\varphi$ and $\psi$ are not in $K$, then $\delta(\varphi) \cup$ $\delta(\psi)=\delta(\varphi \wedge \psi)$.

Proof. As $\dot{-}_{\delta}$ is $K$-grounded and neither $\varphi$ nor $\psi$ are in $K$, it is immediate that

$$
\begin{aligned}
\delta(\varphi) & =\operatorname{dec}(K) \cap \omega(\neg \varphi) \\
\delta(\psi) & =\operatorname{dec}(K) \cap \omega(\neg \psi) \\
\delta(\varphi \wedge \psi) & =\operatorname{dec}(K) \cap \omega(\neg \varphi \vee \neg \psi) .
\end{aligned}
$$

Let us take $\delta(\varphi) \cup \delta(\psi)$. So,

$$
\delta(\varphi) \cup \delta(\psi)=\operatorname{dec}(K) \cap(\omega(\neg \varphi) \cup \omega(\neg \psi))
$$

Notice that $\omega(\neg \varphi \vee \neg \psi)=\omega(\neg \varphi) \cup \omega(\neg \psi)$. So,

$$
\delta(\varphi \wedge \psi)=\operatorname{dec}(K) \cap(\omega(\neg \varphi) \cup \omega(\neg \psi))
$$

Substitution of expression (A.22) in (A.21) give us

$$
\delta(\varphi) \cup \delta(\psi)=\delta(\varphi \wedge \psi)
$$

Lemma A.5. If a choice function is K-grounded, and $\delta(\varphi) \cap \delta(\psi) \neq \emptyset$ then either (i) both $\varphi$ and $\psi$ are in $K$, or (ii) neither $\varphi$ nor $\psi$ are in $K$.

Proof. Let us suppose for contradiction that neither (i) nor (ii) are satisfied. Then, without loss of generality $\varphi \notin K$ and $\psi \in K$. Therefore, as $\delta$ is K-grounded, we have that $\delta(\varphi)=\operatorname{dec}(K) \cap \bar{\omega}(\varphi)(\varphi)$ and $\delta(\psi) \subseteq \bar{\omega}(\psi)$. Thus,

$$
\delta(\varphi) \cap \delta(\psi)=\operatorname{dec}(K) \cap \bar{\omega}(\varphi) \cap \delta(\psi)
$$

As $\psi \in K$, we get that $\operatorname{dec}(K) \cap \bar{\omega}(\psi)=\emptyset$ which implies that $\operatorname{dec}(K) \cap \delta(\psi)=\emptyset$. Therefore,

$$
(\delta(\varphi) \cap \bar{\omega}(\psi)) \cap \delta(\psi)=\emptyset
$$

This implies that $\delta(\varphi) \cap \delta(\psi)=\emptyset$ which is a contradiction. So either (i) or (ii) are satisfied. 
Corollary 3.6. Let $\dot{-}_{\delta}$ be an ECF that satisfies $\left(K_{7}^{-}\right)$and $\left(K_{8}^{-}\right)$, and $\varphi$ and $\psi$ be two non-tautological formulae. If $\delta$ is K-grounded and saturated, and $\delta(\varphi) \cap \delta(\psi) \neq \emptyset$ then $\delta(\varphi \wedge \psi)=\delta(\varphi) \cup \delta(\psi)$.

Proof. Due to $\delta(\varphi) \cap \delta(\psi) \neq \emptyset$, it follows from Lemma A.5 that: either (i) both $\varphi$ and $\psi$ are in $K$, or (ii) neither $\varphi$ nor $\psi$ are in $K$. For the latter, it is straightforward from Observation A.4 that $\delta(\varphi) \cup \delta(\psi)=\delta(\varphi \wedge \psi)$. So we proceed to case (i): both $\varphi$ and $\psi$ are in $K$. As $\dot{-}_{\delta}$ satisfies both $\left(\mathrm{K}_{7}^{-}\right)$and $\left(\mathrm{K}_{8}^{-}\right)$we have From Lemma 3.6 that $\delta(\varphi) \cup \delta(\psi) \subseteq \delta(\varphi \wedge \psi)$ and $\delta(\varphi \wedge \psi) \subseteq \delta(\varphi) \cup \delta(\psi)$. This means that

$$
\delta(\varphi \wedge \psi)=\delta(\varphi) \cup \delta(\psi)
$$

Proposition 3.9. Let $\dot{-}_{\delta}$ be an $E C F, \delta$ be $K$-grounded, and $\varphi$ and $\psi$ be two non-tautological formulae. If $C \notin \delta(\varphi), C \in \delta(\psi)$ and $C \in \delta(\varphi \wedge \psi)$, then $\dot{-}_{\delta}$ does not satisfy (K $\left.K_{7}^{-}\right)$or $\left(K_{8}^{-}\right)$.

Proof. Let us suppose for contradiction that $\dot{-}_{\delta}$ does satisfy $\left(\mathrm{K}_{7}^{-}\right)$and $\left(\mathrm{K}_{8}^{-}\right)$, and that

$$
C \notin \delta(\varphi), C \in \delta(\psi) \text { and } C \in \delta(\varphi \wedge \psi)
$$

As $C \in \delta(\varphi) \cap \delta(\psi)$, it follows that $C \in \bar{\omega}(\varphi) \cap \bar{\omega}(\psi)$ which means that $C$ does not entail $\varphi$. Thus $C$ entails $\neg \varphi$ which means $C$ does entail $\neg \varphi \vee \psi$ which implies that $C \notin \bar{\omega}(\neg \varphi \vee \psi)$ and thus

$$
C \notin \delta(\neg \varphi \vee \psi) \text {. }
$$

Notice that $\varphi \wedge(\neg \varphi \vee \psi) \equiv \varphi \wedge \psi$. Thus, we have from condition (3) of choice function definition that

$$
\delta(\varphi \wedge \psi)=\delta(\varphi \wedge(\neg \varphi \vee \psi))
$$

From C1 of Lemma 3.6, $\delta(\varphi \wedge(\neg \varphi \vee \psi)) \subseteq \delta(\varphi) \cup \delta(\neg \varphi \vee \psi)$ which implies that

$$
C \notin \delta(\varphi \wedge(\neg \varphi \vee \psi)),
$$

because $C \notin \delta(\varphi)$ and $C \notin \delta(\neg \varphi \vee \psi)$. However, from (A.23), $C \in \delta(\varphi \wedge \psi)$ which means $\delta(\varphi \wedge$ $(\neg \varphi \wedge \psi)) \neq \delta(\varphi \wedge \psi)$ which contradicts (A.24).

Lemma 3.7. Given an $E C F \dot{-}_{\delta}$ satisfying $\left(K_{7}^{-}\right)$and $\left(K_{8}^{-}\right)$, and non-tautological formulae $\varphi$ and $\psi$ such that $\delta$ is $K$-grounded. If $\delta(\varphi) \cap \delta(\psi) \neq \emptyset$ then $<_{\varphi} \cup<_{\psi} \subseteq<_{\varphi \wedge \psi}$.

Proof. We need to show that if a pair $(A, B)$ is in $<_{\varphi} \cup<_{\psi}$, then $(A, B) \in<_{\varphi \wedge \psi}$. Let us suppose for contradiction that $(A, B) \in<_{\varphi} \cup<_{\psi}$, but $(A, B) \notin<_{\varphi \wedge \psi}$. As $(A, B) \in<_{\varphi} \cup<_{\psi}$, we have that (i) $(A, B) \in<_{\varphi}$ or (ii) $(A, B) \in<_{\psi}$.

(i) $(A, B) \in<_{\varphi}$. So, from definition of $<_{\varphi}$,

$$
B \in \delta(\varphi) \text { and } A \in(\bar{\omega}(\varphi) \backslash \delta(\varphi))
$$


which means that

$$
A \notin \delta(\varphi) \text { and } A \in \bar{\omega}(\varphi)
$$

As by hypothesis $\delta(\varphi) \cap \delta(\psi) \neq \emptyset$, it follows from Corollary 3.6 that $\delta(\varphi \wedge \psi)=\delta(\varphi) \cup \delta(\psi)$. This implies, as from (A.25) $B \in \delta(\varphi)$, that

$$
B \in \delta(\varphi \wedge \psi)
$$

From definition of $<_{\varphi \wedge \psi}$, a pair $(A, B) \in<_{\varphi \wedge \psi}$ iff $A \notin \delta(\varphi \wedge \psi)$ and $B \in \delta(\varphi \wedge \psi)$. As we have supposed that $(A, B) \notin<_{\varphi \wedge \psi}$, this implies that $A \in \delta(\varphi \wedge \psi)$ or $B \notin \delta(\varphi \wedge \psi)$. However, as $B \in \delta(\varphi \wedge \psi)$, we conclude that $A \in \delta(\varphi \wedge \psi)$. Thus, $A \in \delta(\varphi)$ or $A \in \delta(\psi)$, as $A \in \delta(\varphi \wedge \psi)=\delta(\varphi) \cup \delta(\psi)$. However, from (A.26), $A \notin \delta(\varphi)$, so we conclude that $A \in \delta(\psi)$ which implies that $A \in \delta(\varphi \wedge \psi)$. Summarizing, so far we have that

$$
A \notin \delta(\varphi), A \in \delta(\psi) \text { and } A \in \delta(\varphi \wedge \psi)
$$

This implies through Proposition 3.9 that $\dot{-}_{\delta}$ does not satisfy $\left(\mathrm{K}_{7}^{-}\right)$or $\left(\mathrm{K}_{8}^{-}\right)$which is contradiction as we have assumed that $\dot{-}_{\delta}$ satisfies $\left(\mathrm{K}_{7}^{-}\right)$and $\left(\mathrm{K}_{8}^{-}\right)$.

So, we conclude that $(A, B) \in<_{\varphi \wedge \psi}$.

Case (ii) $(A, B) \in<_{\psi}$ is analogous to case (i). Therefore, we conclude that $<_{\varphi} \cup<_{\psi} \subseteq<_{\varphi \wedge \psi}$.

Theorem 3.7. If an $E C F \dot{-}_{\delta}$ satisfies $\left(K_{7}^{-}\right)$and $\left(K_{8}^{-}\right)$, and $\delta$ is $K$-grounded, then the choice function $\delta$ is an annulment.

Proof. Let $\dot{-}_{\delta}$ be an ECF that satisfies $\left(\mathrm{K}_{7}^{-}\right)$and $\left(\mathrm{K}_{8}^{-}\right)$, and $\delta$ a $K$-grounded choice function. Moreover, let $<$ be the shadow of $\delta$ and $\mu_{<}$an annulment. We need to prove that, $\delta$ and $\mu_{<}$are equivalent. First of all, an annulment consults $<$ only for non-tautological formulae. So, if $\varphi$ is tautological it suffices to make $\mu_{<}(\varphi)=\delta(\varphi)$. We proceed for the case that $\varphi$ is not tautological. Let us suppose, for contradiction, that $\mu_{<}$is not equivalent to $\delta$. So, for some non-tautological formula $\varphi, \mu_{<}(\varphi) \neq \delta(\varphi)$. This means that, there is a theory $A$ in $\delta(\varphi)$ that is not maximal in $\bar{\omega}(\varphi)$ w.r.t $<$. In other words,

$$
\text { there is }(A, B) \in<\text {, s.t } A, B \in \bar{\omega}(\varphi) \text { and } A \in \delta(\varphi) \text {. }
$$

From definition of shadow, we have that a pair $(A, B) \in<\operatorname{iff}(A, B) \in<_{\varphi}$ or for some non-tautological formula $\psi,(A, B) \in<_{\psi}$ or $(A, B) \in \nabla(\varphi, \psi) \cup \nabla(\psi, \varphi)$. For the latter, $(A, B) \in \nabla(\varphi, \psi)$ or $(A, B) \in$ $\nabla(\psi, \varphi)$. In either case, $A \notin \delta(\varphi)$ which is a contradiction as $A \in \delta(\varphi)$. Assuming $(A, B) \in<_{\varphi}$ is also a contradiction, because from definition $(A, B) \in<_{\varphi}$ means that $A \notin \delta(\varphi)$, however we have supposed that $A \in \delta(\varphi)$. So, we assume that $(A, B)$ has to be in $<_{\psi}$ which from its definition implies that $B \in \delta(\psi)$ and $A \in \bar{\omega}(\psi)$. Summarizing,

$$
(A, B) \in<_{\psi}, B \in \delta(\psi) \text { and } A \in \bar{\omega}(\psi)
$$

As from (A.29) $B \in \bar{\omega}(\varphi)$, we have two options to consider: (i) $B \in \delta(\varphi)$ or (ii) $B \notin \delta(\varphi)$ : 
(i) $B \in \delta(\varphi)$. So, we have that $\delta(\varphi) \cap \delta(\psi) \neq \emptyset$, as from (A.30) $B \in \delta(\psi)$. This implies from from Corollary 3.6 that $\delta(\varphi \wedge \psi)=\delta(\varphi) \cup \delta(\psi)$. Then, both $A$ and $B$ belongs to $\delta(\varphi \wedge \psi)$, as $B \in \delta(\psi)$ and from (A.29) $A \in \delta(\varphi)$. It follows from definition of $<_{\varphi \wedge \psi}$ that a pair $(A, B) \in<_{\varphi \wedge \psi}$ iff $A \notin \delta(\varphi \wedge \psi)$ and $B \in \delta(\varphi \wedge \psi)$. However, as $A \in \delta(\varphi \wedge \psi)$, we conclude that $(A, B) \notin<_{\varphi \wedge \psi}$. As $\delta(\varphi) \wedge \delta(\psi) \neq \emptyset$ and $\dot{-}_{\delta}$ satisfies $\left(\mathrm{K}_{7}^{-}\right)$and $\left(\mathrm{K}_{8}^{-}\right)$, it follows from Lemma 3.7 that $<_{\varphi} \cup<_{\psi} \subseteq<_{\varphi \wedge \psi}$. Thus, as $(A, B) \notin<_{\varphi \wedge \psi}$, we have that $(A, B)$ does not belong either to $<_{\varphi}$ nor to $<_{\psi}$ which is a contradiction as, from (A.30), $(A, B) \in<_{\psi}$.

(ii) $B \notin \delta(\varphi)$. As from (A.29) $B \in \bar{\omega}(\varphi)$ and $A \in \delta(\varphi)$, it follows from $<_{\varphi}$ definition that $(B, A) \in<_{\varphi}$. From C1 of Lemma 3.6, $\delta(\varphi \wedge \psi) \subseteq \delta(\varphi) \cup \delta(\psi)$ which means that for each theory $S$ of $\delta(\varphi \wedge \psi), S \in \delta(\varphi)$ or $S \in \delta(\psi)$. Therefore, from C2 of Lemma 3.6, we have that: (1.) $\delta(\varphi) \subseteq \delta(\varphi \wedge \psi)$ or $(2.) \delta(\psi) \subseteq \delta(\varphi \wedge \psi)$.

1. $\delta(\varphi) \subseteq \delta(\varphi \wedge \psi)$. So, $A \in \delta(\varphi \wedge \psi)$, which implies from definition of $<_{\varphi \wedge \psi}$ that $(A, B) \notin<_{\varphi \wedge \psi}$. We have to cases to consider: (a) $\delta(\varphi) \cap \delta(\psi) \neq \emptyset$ or (b) $\delta(\varphi) \cap \delta(\psi=\emptyset$.

(a) If $\delta(\varphi) \cap \delta(\psi) \neq \emptyset$, then $<_{\varphi} \cup<\psi \subseteq<_{\varphi \wedge \psi}$. Thus, as $(A, B) \notin<_{\varphi \wedge \psi}$, then $(A, B)$ does not belong either to $<_{\varphi}$ nor to $<_{\psi}$, which contradicts $(A, B) \in<\psi$.

(b) $\delta(\varphi) \cap \delta(\psi)=\emptyset$. So, as $A \in \delta(\varphi)$, then $A \notin \delta(\psi)$. Notice that if some $S$ of $\delta(\psi)$ is in $\delta(\varphi \wedge \psi)$, then

$$
\delta(\varphi \wedge \psi)=\delta(\varphi) \cup \delta(\psi)
$$

Then, we have two cases: $\delta(\varphi \wedge \psi)=\delta(\varphi) \cup \delta(\psi)$ or $\delta(\varphi)=\delta(\varphi \wedge \psi)$, since $\delta(\varphi) \subseteq \delta(\varphi \wedge \psi)$. For the former, as $A \in \delta(\varphi)$ and $A \notin \delta(\psi)$ we have that $A \in \delta(\varphi \wedge \psi)$. Thus, from Proposition 3.9 , we have that $\dot{-}_{\delta}$ does not satisfy $\left(\mathrm{K}_{7}^{-}\right)$or $\left(\mathrm{K}_{8}^{-}\right)$which is a contradiction. So it only rest to us to assume that $\delta(\varphi)=\delta(\varphi \wedge \psi)$. Summarizing, so far we have

$$
A \in \delta(\varphi), B \in \delta(\psi), A \in \delta(\varphi \wedge \psi) \text { and } B \notin \delta(\varphi \wedge \psi)
$$

As A belongs to both $\bar{\omega}(\varphi)$ and $\bar{\omega}(\psi)$, we have that $A$ does not entail either $\varphi$ nor $\psi$, which means $A$ entails $\neg \psi \vee \varphi$. Thus, $A \notin \bar{\omega}(\neg \psi \vee \psi)$. Now notice that the formulae $\psi \wedge(\neg \psi \vee \varphi) \equiv$ $\varphi \wedge \psi$. As $\delta$ is a choice function, this implies that

$$
\delta(\psi \wedge(\neg \psi \vee \varphi))=\delta(\varphi \wedge \psi)
$$

From conditions $\mathrm{C} 1$ and $\mathrm{C} 2$ of Lemma 3.6 we have the two following scenarios: (i) if some $S$ of $\delta(\psi)$ is chosen, then $S$ belongs to $\delta(\varphi \wedge \psi)$ as well; (ii) analogously, if $S \in \delta(\neg \psi \vee \varphi)$ that is also belongs to $\delta(\varphi \wedge \psi)$. For the former, $B \in \delta(\varphi \wedge \psi)$ which is a contradiction as $B \notin \delta(\varphi \wedge \psi)$. For the latter, $A \notin \delta(\varphi \wedge \psi)$ which is also a contradiction, as $A \in \delta(\varphi \wedge \psi)$.

2. $\delta(\psi) \subseteq \delta(\varphi \wedge \psi)$. Analogous to item (I). 
Therefore, we conclude that $\mu_{<}$is equivalent to $\delta$.

Theorem 3.8. Let $\dot{-}_{\delta}$ be an ECF that satisfies $\left(K_{7}^{-}\right)$and $\left(K_{8}^{-}\right)$. If $\delta$ is K-grounded, then the shadow $<$ of $\delta$ satisfies mirroring.

Proof. Let $\dot{-}_{\delta}$ be a ECF that satisfies $\left(\mathrm{K}_{7}^{-}\right)$and $\left(\mathrm{K}_{8}^{-}\right)$, and $<$the shadow of $\delta$. Let us suppose, for contradiction, that $<$ does not satisfy mirroring. So, for some complete theories $A, B$ and $M$,

$$
A \nless B \text { and } B \nless A \text { and } A<M \text { and } B \nless M \text {. }
$$

As $A<M$, and $<$ is the shadow of $\delta$, we have from $<$ definition that:

$$
\text { (i) }(A, M) \in<_{\varphi} \text { or }(i i)(A, M) \in \nabla(\varphi, \psi) \cup \nabla(\psi, \varphi) \text {. }
$$

(i) $(A, M) \in<_{\varphi}$. Then, from definition of $<_{\varphi}$,

$$
M \in \delta(\varphi), \text { and } A, M \in \bar{\omega}(\varphi)
$$

First we will need to notice that $B \notin \bar{\omega}(\varphi)$. To see this, let us suppose, for contradiction, that $B \in \bar{\omega}(\varphi)$. Then, two cases arises: $B \in \delta(\varphi)$ or $B \notin \delta(\varphi)$. In either cases, we have that $A<B$ or $B<M$, which is a contradiction as we have assumed that $A \nless B$ and $B \nless M$. Therefore,

$$
B \notin \bar{\omega}(\varphi)
$$

Now we proceed with the proof. Let $\psi$ be a formula that is not entailed by $B$, then

$$
B \in \bar{\omega}(\psi)
$$

We have two cases to consider: $\delta(\varphi) \cap \delta(\psi) \neq \emptyset$ or $\delta(\varphi) \cap \delta(\psi)=\emptyset$.

- $\delta(\varphi) \cap \delta(\psi) \neq \emptyset$ then, from Corollary 3.6, $\delta(\varphi \wedge \psi)=\delta(\varphi) \cup \delta(\psi)$ which implies that

$$
M \in \delta(\varphi \wedge \psi)
$$

because $M \in \delta(\varphi)$.

We recall that, $\bar{\omega}(\varphi \wedge \psi)=\bar{\omega}(\varphi) \cup \bar{\omega}(\psi)$. Thus, as $A, M \in \bar{\omega}(\varphi)$ and $B \in \bar{\omega}(\psi)$, we have that $A, B, M \in \bar{\omega}(\varphi \wedge \psi)$. So, either: (a) $B<M$, if $B \notin \delta(\psi)$; or (b) $A<B$, if $B \in \delta(\psi)$.

(a) $B \notin \delta(\psi)$. Then, as $B \notin \delta(\varphi)$ and $\delta(\varphi \wedge \psi)=\delta(\varphi) \cup \delta(\psi)$, we have

$$
B \notin \delta(\varphi \wedge \psi) .
$$

Moreover, $M \in \delta(\varphi \wedge \psi)$. Thus, from definition, $(B, M) \in<_{\varphi \wedge \psi}$ which means $B<M$ which is a contradiction, as we have assumed that $B \nless M$.

(b) $B \in \delta(\psi)$. We have that $A \in \delta(\psi)$ or $A \notin \delta(\psi)$. In either cases, $A \nless M$ or $A<B$ which is a contradiction, as by hypothesis $A<M$ and $A \nless B$. 
- $\delta(\varphi) \cap \delta(\psi)=\emptyset$. If we assume that $\delta(\varphi \wedge \psi)=\delta(\varphi) \cup \delta(\psi)$, then we will get the same contradiction as in the previous case. Thus, we assume $\delta(\varphi \wedge \psi) \neq \delta(\varphi) \cup \delta(\psi)$. We have two cases to consider: $B \in \delta(\psi)$ or $B \notin \delta(\psi)$. For the former case, $A \in \delta(\psi)$ or $A \notin \delta(\psi)$. In either cases, $A \nless M$ and $A<B$ which is a contradiction as by hypothesis $A<M$ and $A \nless B$. Then, it only rest for us to assume that $B \notin \delta(\psi)$. Clearly, $B \notin \delta(\varphi)$, as $B \notin \bar{\omega}(\varphi)$. This implies that $B \in \bar{\omega}(\psi) \backslash \delta(\varphi)$. Recall that $M \in \delta(\varphi)$, thus from triangulation $(B, M) \in \nabla(\psi, \varphi)$. This implies that $B<M$ which is a contradiction as by hypothesis $B \nless M$. Thus, we conclude

$$
(A, M) \notin<_{\varphi}
$$

(ii) (a) $(A, M) \in \nabla(\varphi, \psi)$ or (b) $(A, M) \in \nabla(\psi, \varphi)$.

(a) Let us suppose that $(A, M) \in \nabla(\varphi, \psi)$, then

$$
A \in \bar{\omega}(\varphi), A \notin \delta(\varphi), A \notin \bar{\omega}(\psi) \operatorname{and} M \in \delta(\psi) .
$$

This implies that,

$$
A \in(\bar{\omega}(\varphi) \backslash(\delta(\varphi) \cup \bar{\omega}(\varphi))
$$

Let $\alpha \in((A \backslash M) \backslash B)$, that is, $\alpha$ is a formula entailed by $A$ but that is not entailed either by $M$ nor $B$. Thus,

$$
A \notin \bar{\omega}(\alpha) \text { and } B, M \in \bar{\omega}(\alpha) \text {. }
$$

There are three possible cases w.r.t $B$ we have to consider:

(1) $B \in \delta(\psi)$, or

(2) $B \in \delta(\alpha)$, or

(3) otherwise, that is, $B \notin \delta(\psi)$ and $B \notin \delta(\alpha)$.

For (1), $B \in \delta(\psi)$, which together with (A.36) implies through triangulation definition that $(A, B) \in \nabla(\varphi, \psi)$. Thus, $A<B$ which is a contradiction, as by hypothesis $A \nless B$.

For (2), assume that $B \in \delta(\alpha)$. From (A.35) and (A.37), we have

$$
A \in \bar{\omega}(\varphi), A \in A \notin \delta(\varphi) \text { and } A \notin \bar{\omega}(\alpha) \text {, }
$$

which means that $A \in(\bar{\omega}(\varphi \backslash(\delta(\varphi \cup \bar{\omega}(\alpha)))$. Thus, as $B \in \delta(\alpha)$, we have $(A, B) \in \nabla(\varphi, \alpha)$ which also implies $A<B$ which is a contradiction, as by hypothesis $A \nless B$.

For (3), assume that $B \notin \delta(\psi)$ and $B \notin \delta(\alpha)$. Thus, we $B \in \bar{\omega}(\alpha) \backslash \delta(\psi)$ which implies that $(B, M) \in \nabla(\alpha, \psi)$, as $M \in \delta(\psi)$. Then, $B<M$ which is also a contradiction as by hypothesis $B \nless M$.

As (1), (2) and (3) all leads to contradictions, we conclude that $(A, M) \notin \nabla(\varphi, \psi)$. The case (b) $(A, M) \in \nabla(\psi, \varphi)$ is analogous. So we conclude that, 


$$
(A, M) \notin \nabla(\varphi, \psi) \text { and }(A, M) \notin \nabla(\psi, \varphi) \text {. }
$$

So, from (A.34) and (A.38) we have that $(A, M) \notin<_{\varphi}$ and $(A, M) \notin \nabla(\varphi, \psi) \cup \nabla(\psi, \varphi)$ which contradictions (A.33). Thus, we conclude that $<$ does satisfy mirroring.

Corollary 3.9. Every contraction function that satisfies all the eight postulates is equivalent to $a B C F$.

Proof. As it satisfies the six basic postulates, we have that it is equivalent to an ECF over a CF $\delta$. We have from Observation 3.7 that there is a $K$-grounded choice function $\delta^{\prime}$ such that $\dot{-}_{\delta}$ is equivalent to $\dot{-}_{\delta^{\prime}}$. So, we only need to prove that $\delta^{\prime}$ is an annulment. Let $<$ be the shadow of $\delta^{\prime}$. Thus, from Theorem 3.7 we have that $\mu_{<}$is equivalent to $\delta^{\prime}$. Moreover, from Theorem 3.8 we have that $<$ satisfies mirroring. Furthermore, maximal cut follows directly from its definition. So, such a function is a BCF. 
APPENDIX A 


\section{Appendix B}

\section{Proofs for Chapter 3, Section 3.4}

Theorem 3.10. Every ERF satisfies $\left(K_{1}^{*}\right)-\left(K_{6}^{*}\right)$.

Proof. Let $*_{\delta}$ be a ERF, $K$ a theory and $\varphi$ a formula. The case $\left(\mathbf{K}_{1}^{*}\right)$ is trivial, so we focus on the others.

$\left(\mathbf{K}_{2}^{*}\right)$ Trivially, $\varphi \in K+\varphi$. For the another case, every theory in $\delta(\varphi)$ entails $\varphi$. Therefore, $\varphi \in \bigcap \delta(\varphi)$.

$\left(\mathbf{K}_{3}^{*}\right)$ There is nothing to prove for the case $K *_{\delta} \varphi=K+\varphi$. Thus, let us suppose that $K *_{\delta}$ $\varphi=\bigcap \delta(\varphi)$. From definition, $\perp \in K+\varphi$. Therefore, $\neg \varphi \in K$. This means that $K+\varphi=C n(\perp)$ which means that $K *_{\delta} \varphi \subseteq K+\varphi$.

( $\left.\mathbf{K}_{4}^{*}\right)$ Let us suppose that $\neg \varphi \notin K$. Then, $K *_{\delta} \varphi=K+\varphi$ which implies that $K+\varphi \subseteq K *_{\delta} \varphi$.

$\left(\mathbf{K}_{5}^{*}\right)$ The direction " $\left.\Leftarrow\right)$ " is trivial, so we consider the another direction.

" $(\Rightarrow)$ " Let us suppose that $K *_{\delta} \varphi=C n(\perp)$. Then, from definition of ERF, either $K *_{\delta} \varphi=$ $K+\varphi$ or $K *_{\delta} \varphi=\bigcap \delta(\varphi)$. Clearly, the second case cannot be satisfied, since $\delta$ selects only consistent complete theories which implies that $\bigcap \delta(\varphi) \subset C n(\perp)$, that is, $\bigcap \delta(\varphi) \subset K *_{\delta} \varphi$. This leave us only with the first case, that is, $K *_{\delta} \varphi=K+\varphi$. From definition of ERF, we have that $K+\varphi \neq C n(\perp)$ or $C n(\varphi)=C n(\perp)$. Since, from hypothesis $K *_{\delta} \varphi=C n(\perp)$, we get that $C n(\varphi)=C n(\perp)$.

$\left(\mathbf{K}_{6}^{*}\right)$ Let $C n(\varphi)=C n(\psi)$. Thus, either $K *_{\delta} \varphi=K+\varphi$ or $K *_{\delta} \varphi=\bigcap \delta(\varphi)$. For the former, $K+\varphi=K+\psi$. For the latter, it follows from the condition (3) of a choice function that $\delta(\varphi)=\delta(\psi)$. Thus, $\bigcap \delta(\varphi)=\bigcap(\psi)$, that is, $K * \varphi=K * \psi$.

Proposition 3.10. Let $*$ be a revision function that satisfies $\left(K_{1}^{*}\right)-\left(K_{6}^{*}\right) ; \perp \notin K+\varphi$ or $C n(\varphi)=$ $C n(\perp)$ iff $K * \varphi=K+\varphi$.

Proof. " $\Rightarrow$ " Let us suppose that $\perp \notin K+\varphi$ or $C n(\varphi)=C n(\perp)$. We have to show that $K * \varphi=K+\varphi$.

- Let us suppose that $\perp \notin K+\varphi$. Then, $\varphi \rightarrow \perp \notin K$ which implies that $\neg \varphi \notin K$. Thus, from $\left(\mathbf{K}_{4}^{*}\right)$, we have that $K+\varphi \subseteq K * \varphi$ which together with $\left(\mathbf{K}_{3}^{*}\right)$ implies that $K * \varphi=K+\varphi$. 
- Let us suppose that $C n(\varphi)=C n(\perp)$. Thus, $K+\varphi=C n(\perp)$ and $\neg \varphi \in C n(\emptyset)$ which implies from $\left(\mathbf{K}_{5}^{*}\right)$ that $K * \varphi=C n(\perp)$. Therefore, $K * \varphi=K+\varphi$.

" $\Leftarrow$ " Let us suppose for contradiction that $K * \varphi=K+\varphi$, but $\perp \in K+\varphi$ and $C n(\varphi) \neq C n(\perp)$. It follows from $\perp \in K+\varphi$ that $K+\varphi=C n(\perp)$. Thus, as $K * \varphi=K+\varphi$, we have

$$
K * \varphi=C n(\perp) .
$$

It follow from $C n(\varphi) \neq C n(\perp)$ that $\neg \varphi \notin C n(\emptyset)$ which implies from $\left(\mathbf{K}_{5}^{*}\right)$ that $K * \varphi \neq C n(\perp)$. This however contradicts (B.1). This ends the proof.

Observation B.1. If $\varphi \in K$ then $K * \varphi=K+\varphi$, for every AGM rational revision function $*$.

Proof. It is straightfowrd from the $\left(\mathrm{K}_{3}^{*}\right)$ and $\left(\mathrm{K}_{4}^{*}\right)$.

Theorem 3.11. If a revision function satisfies the six basic postulates, then it is equivalent to some ERV.

Proof. Let $*$ a revision function that satisfies $\left(\mathbf{K}_{1}^{*}\right)$ to $\left(\mathbf{K}_{6}^{*}\right)$.

According to Lemma 3.2, every consistent theory $K$ can be written as the intersection of a collection of consistent complete theories, that is, $K=\bigcap \operatorname{dec}(K)$. This means that we can construct a casting function from $d e c$ as follows:

$$
\delta(\varphi)=\left\{\begin{array}{cl}
\operatorname{dec}(K * \varphi) & \text { if } C n(\varphi) \neq C n(\perp) \\
T_{L} & \text { otherwise. }
\end{array}\right.
$$

It is easy to check that $\delta$ is indeed a casting function. First, notice that for every formula $\varphi, \delta(\varphi) \neq \emptyset$. For condition (2) and (3), take two formulae $\varphi$ and $\psi$ logically equivalent, that is, $C n(\varphi)=C n(\psi)$. Clearly, if $\varphi \neq C n(\perp)$, then $\operatorname{dec}(\varphi)=\operatorname{dec}(\psi)$ which contains only consistent complete theories that entail $\varphi$ (respectively $\psi$ ); and if $C n(\varphi)=C n(\perp)$, then $\delta(\varphi)=\delta(\psi)=T_{L}$. This proofs that $\delta$ is a casting function.

Now, we will construct a revision function $*_{\delta}$ founded in the function $\delta$. Then, we will show that $K * \varphi=K *_{\delta} \varphi$.

$$
K *_{\delta} \varphi= \begin{cases}K+\varphi & \text { if } \perp \notin K+\varphi \text { or } C n(\varphi)=C n(\perp) \\ \bigcap \delta(\varphi) & \text { w.w }\end{cases}
$$

Is is straightforward from its definition that $*_{\delta}$ is an ERF. As both $*_{\delta}$ and $*$ are ERV, both satisfies the six basic AGM revision postulates. Thus, from Proposition 3.10, we have that

$$
\begin{aligned}
K * \varphi & =K+\varphi \text { iff } \perp \notin K+\varphi \text { or } C n(\varphi)=C n(\perp) \\
K *_{\delta} \varphi & =K+\varphi \text { iff } \perp \notin K+\varphi \text { or } C n(\varphi)=C n(\perp)
\end{aligned}
$$

Thus,

$$
K * \varphi=K+\varphi \text { iff } \perp \notin K+\varphi \text { or } C n(\varphi)=C n(\perp) .
$$


So, to complete the demonstration that $K * \varphi=K *_{\delta} \varphi$, we just need to show what happens when $\perp \in K+\varphi$ and $C n(\varphi) \neq C n(\perp)$. It follows from definition of $\delta$ that $\delta(\varphi)=\operatorname{dec}(K * \varphi)$. It follows from Lemma 3.2 that $K * \varphi=\bigcap \operatorname{dec}(K * \varphi)$. This implies that, $\bigcap \delta(\varphi)=\bigcap \operatorname{dec}(K * \varphi)=K * \varphi$. Thus,

$$
\begin{aligned}
K *_{\delta} \varphi & =\bigcap \delta(\varphi) \\
& =K * \varphi .
\end{aligned}
$$

Therefore,

$$
K *_{\delta} \varphi=K * \varphi, \text { if } \perp \in K+\varphi \text { and } C n(\varphi) \neq C n(\perp) .
$$

Therefore, from (B.2) and (B.3), we conclude that $K * \varphi=$ and $K *_{\delta} \varphi$.

Lemma B.1. If there is a $S \in \nu_{<}(\varphi)$ such that $\varphi \wedge \psi \in S$, then $S \in \nu_{<}(\varphi \wedge \psi)$.

Proof Sketch: We recall that $\nu_{<}(\varphi)=\max _{<}(\omega(\varphi))$, that is the maximal elements of $\omega(\varphi)$ ordered via a binary relation $<$. Let us suppose that there is a complete theory $S \in \nu_{<}(\varphi)$ such that $\varphi \wedge \psi \in S$. This means that $S \in \omega(\varphi \wedge \psi)$ and $S$ is maximal element of $\omega(\varphi)$. The latter implies that $S$ is a maximal element of any subset of $\omega(\varphi)$. Therefore, as $\omega(\varphi \wedge \psi) \subseteq \omega(\varphi)$, we conclude that $S$ is a maximal element of $\omega(\varphi \wedge \psi)$, that is, $S \in \nu_{<}(\varphi \wedge \psi)$.

Proof. We recall that $\nu_{<}(\varphi)=\max _{<}(\omega(\varphi))$, that is the maximal elements of $\omega(\varphi)$ ordered via a binary relation $<$. Let us suppose that there is a complete theory $S \in \nu_{<}(\varphi)$ such that $\varphi \wedge \psi \in S$. This means that

$$
\text { for all } S^{\prime} \in \omega(\varphi), S \nless S^{\prime} \text {. }
$$

From $\varphi \wedge \psi$, we get that $S \in \omega(\varphi \wedge \psi)$. As $\omega(\varphi \wedge \psi)=\omega(\varphi) \cap \omega(\psi)$, we know that $\omega(\varphi \wedge \psi) \subseteq \omega(\varphi)$ which from (B.4) implies that

$$
\text { for all } S^{\prime} \in \omega(\varphi \wedge \psi), S \nless S^{\prime} \text {. }
$$

Therefore, $S \in \max _{<}(\omega(\varphi \wedge \psi))$, that is, $S \in \nu(\varphi \wedge \psi)$.

Lemma B.2. Let $A$ be a collection of complete consistent theories. If $\alpha \in \bigcap(A \cap \omega(\psi))$, then $\psi \rightarrow \alpha \bigcap A$.

Proof. Let $\alpha$ be a formula in $\bigcap(A \cup \omega(\psi))$. The collection $\omega(\psi)$ contains all the consistent complete theories that entail $\psi$. Therefore $A \cap \omega(\psi)$ contains all the consistent complete theories from $A$ that entail $\psi$. As $\alpha \in \bigcap(A \cup \omega(\psi))$, it implies that every consistent complete theory of $A$ that entails $\psi$ also entails $\alpha$, that is,

$$
\text { for all } S \in A \text {, if } \psi \in S \text { then } \alpha \in S \text {. }
$$

Given a $S \in A$, we have two cases to consider: 
(i) $\psi \notin S$. Then, $\neg \psi \in S$, as $S$ is a complete theory. Therefore, $\psi \rightarrow \alpha \in S$.

(ii) $\psi \in S$. Then, from (B.6), $\alpha \in S$ which implies that $\psi \rightarrow \alpha \in S$.

Thus, from (i) and (ii), we conclude that for every $S \in A, \psi \rightarrow \alpha \in S$. Therefore, $\psi \rightarrow \alpha \in \bigcap A$.

Proposition 3.11. Let $K$ be a theory, $\psi$ a formula and $A$ a set of complete theories such that $K=\bigcap A$. If $K+\psi$ is consistent then $K+\psi=\bigcap(A \cap \omega(\psi))$.

Proof. As $K+\psi$ is consistent and $K=\bigcap A$, we get that $A$ has at least one consistent complete theory that entails $\psi$. This means that $A \cap \omega(\psi) \neq \emptyset$, as $\omega(\psi)$ contains all the consistent complete theories that entail $\psi$. As $A \cap \omega(\psi) \subseteq A$, we get that $\bigcap(A) \subseteq \bigcap(A \cap \omega(\psi))$. Thus, as $\bigcap A=K$,

$$
K \subseteq \bigcap(A \cap \omega(\psi))
$$

We will need the expression above later on this proof.

To show that $\bigcap(A \cap \omega(\psi))=K+\psi$, we will show that $\bigcap(A \cap \omega(\psi)) \subseteq K+\psi$ and thereafter $K+\psi \subseteq \bigcap(A \cap \omega(\psi))$.

" $\Rightarrow)$ " We will show that $K+\psi \subseteq \bigcap(A \cap \omega(\psi))$.

As by hypothesis $K+\psi$ is consistent, we have that $\neg \psi$ is in none of the consistent complete theories of $A$. This means that some consistent complete theory of $A$ entail $\psi$. Therefore, $\bigcap(A \cap$ $\omega(\psi)) \neq \emptyset$. Let $\alpha \in K+\psi$, we will show that $\alpha \in(\bigcap(A \cap \omega(\psi))$. Thus, $\psi \rightarrow \alpha \in K$ which from (B.7) implies that $\psi \rightarrow \alpha \in(\bigcap(A \cap \omega(\psi))$. All the consistent complete theories in $A \cap \omega(\psi)$ entails $\psi$, which means that $\psi \in \bigcap(A \cap \omega(\psi))$. Therefore, $\alpha \in \bigcap(A \cap \omega(\psi))$.

" $(\Leftarrow)$ " We will show that $\bigcap(A \cap \omega(\varphi)) \subseteq K+\varphi$. Let $\alpha \in \bigcap(A \cap \omega(\psi))$, then from Lemma B.2 $\psi \rightarrow \alpha \in \bigcap A$. This implies that $\psi \rightarrow \alpha \in K$, as $K=\bigcap A$. Therefore, $\alpha \in K+\psi$.

Theorem 3.12. Every mirrored CRF satisfies $\left(K_{7}^{*}\right)$.

Proof. Let $*$ be a CRF, and $K$ a theory. We have to show that for every formulae $\varphi$ and $\psi, K * \varphi \wedge \psi \subseteq$ $K * \varphi+\psi$. We have two main cases to consider: either $K * \varphi+\psi=C n(\perp)$ or $K * \varphi+\psi \neq C n(\perp)$. There is nothing to prove for the former, as it states directly that $K * \varphi \wedge \psi \subseteq K * \varphi+\psi$, as every theory is a subset of $C n(\perp)$. Thus we just need to check the case $K * \varphi+\psi \neq C n(\perp)$. This means that $\perp \notin K * \varphi+\psi$ which implies

$$
\begin{array}{r}
\varphi \not \equiv \perp \text { and } \psi \not \equiv \perp ; \\
\neg \psi \notin K * \varphi .
\end{array}
$$

According to the CRF definition, either (i) $K * \varphi \wedge \psi=K * \varphi \wedge \psi$ or (ii) $K * \varphi \wedge \psi=\bigcap \mu_{<}(\varphi \wedge \psi)$. We recall that every CRF is an ERF. 
(i) $K * \varphi \wedge \psi=K+\varphi \wedge \psi$. Thus, from ERF definition $\perp \notin K+\varphi \wedge \psi$ or $\varphi \wedge \psi \equiv \perp$. However, as $\varphi \not \equiv \perp$ and $\psi \not \equiv \perp$, this implies that $\perp \notin K+\varphi \wedge \psi$. Therefore, $\neg \varphi \vee \neg \psi \notin K$ which means

$$
\neg \varphi \notin K \text { and } \neg \psi \notin K \text {. }
$$

Thus, $K * \varphi=K+\varphi$. Moreover, as $K+\varphi \wedge \psi=(K+\varphi)+\psi$, we have that $K+\varphi \wedge \psi=K * \varphi+\psi$ which implies $K * \varphi \wedge \psi=K * \varphi+\psi$. Therefore, $K * \varphi \wedge \psi \subseteq K * \varphi+\psi$.

(ii) $K * \varphi \wedge \psi=\bigcap \mu_{<}(\varphi \wedge \psi)$. This implies from ERF definition that $\neg \varphi \vee \neg \psi \in K$. There are two possible cases to consider: $K * \varphi=K+\varphi$ or $K * \varphi=\bigcap \mu_{<}(\varphi)$. For the former, $K * \varphi+\psi=K+\varphi+\psi$ which implies that $K * \varphi+\psi=K+\varphi \wedge \psi$. Thus, from ( $\mathbf{K}_{3}^{*}$ ) we have that $K * \varphi \wedge \psi \subseteq K+\varphi \wedge \psi$, which gives us that $K * \varphi \wedge \psi \subseteq K * \varphi+\psi$. Now, there is only the second case left to be checked, that is, $K * \varphi=\bigcap \mu_{<}(\varphi)$. This implies from (B.9) that there is a $S \in \mu_{<}(\varphi)$ such that $\neg \psi \notin S$. As the logic is closed under classical negation, this means that $\psi \in S$. As every theory of $\mu_{<}(\varphi)$ entails $\varphi$, we have that $\varphi \wedge \psi \in S$, which from Lemma B.1 implies that $S \in \mu_{<}(\varphi \wedge \psi)$. Therefore, $\mu_{<}(\varphi) \cap \omega(\psi) \subseteq \mu_{<}(\varphi \wedge \psi)$. Thus,

$$
\bigcap \mu_{<}(\varphi \wedge \psi) \subseteq \bigcap\left(\mu_{<}(\varphi) \cap \omega(\psi)\right)
$$

As, from Proposition 3.11, $K * \varphi+\psi=\bigcap\left(\mu_{<}(\varphi) \cap \omega(\psi)\right)$, it follows that

$$
K * \varphi \wedge \psi \subseteq K * \varphi+\psi
$$

Observation B.2. If $\neg \psi \notin K * \varphi$ and $\neg \varphi \vee \neg \psi \in K$, then $K * \varphi \neq K+\varphi$

Proof. Let us suppose for contradiction that $\neg \psi \notin K * \varphi$ and $\neg \varphi \vee \neg \psi \in K$, but $K * \varphi=K+\varphi$. Therefore, $\neg \psi \notin K+\varphi$. This implies that $\varphi \rightarrow \neg \psi \notin K$, that is, $\neg \varphi \vee \neg \psi \notin K$ which contradicts out hypothesis that $\neg \varphi \vee \neg \psi \in K$.

Theorem 3.13. Every CRF satisfies ( $\left.K_{8}^{*}\right)$.

Proof. Let us suppose that $\neg \psi \notin K * \varphi$.

The case $K * \varphi \wedge \psi=K+\varphi \wedge \psi$ is analogous to this same case for $\left(\mathrm{K}_{7}^{*}\right)$. So we will not repeat the same proof here. We are left then with the case $K * \varphi \wedge \psi=\mu_{<}(\varphi \wedge \psi)$. This implies, from the definition of CRF that $K+\varphi \wedge \psi=L$. Therefore, $\neg \varphi \vee \neg \psi \in K$ which together with our hypothesis that $\neg \psi \notin K * \varphi$ implies from Observation B.2 that $K * \varphi \neq K+\varphi$. Therefore, according to CRF definition: $K * \varphi=\bigcap \mu_{<}(\varphi)$.

- $K * \varphi=\bigcap \mu_{<}(\varphi)$. From hypothesis $\neg \psi \notin K * \varphi$, which implies that there is a theory $S \in$ $\mu_{<}(\varphi)$ that entails $\psi$. This means that $S \in \delta(\varphi) \cap \omega(\psi)$. Let us suppose for contradiction

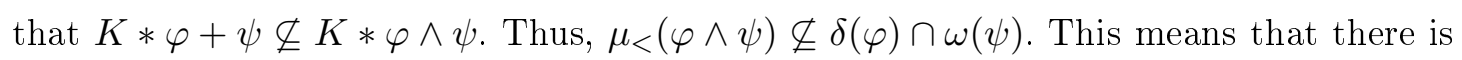
some $S^{\prime} \in \mu_{<}(\varphi \wedge \psi)$ such that $S^{\prime} \notin \mu_{<}(\varphi) \cap \omega(\psi)$. As every complete consistent theory in $\mu_{<}(\varphi \wedge \psi)$ entails both $\varphi$ and $\psi$, we get that $S^{\prime}$ are in both $\omega(\psi)$ and $\omega(\varphi)$. Therefore, 
we get that $S^{\prime} \notin \mu_{<}(\varphi)$. This implies that for some $M \in \omega(\varphi), S^{\prime} \leq M$. Moreover, as $S^{\prime} \in \mu_{<}(\varphi \wedge \psi)$, we get that for every $M^{\prime} \in \omega(\varphi \wedge \psi), S^{\prime} \not \leq M^{\prime}$. This means that $M \notin \omega(\varphi \wedge \psi)$. Therefore, $M \in \omega(\varphi)$. So far, we have that $S \nless S^{\prime}$ and $S^{\prime} \nless S$, as both are in $\mu_{<}(\varphi \wedge \psi)$. However, as $S^{\prime} \leq M$, it follows from mirroring that $S \leq M$ which is a contradiction.

Observation 3.11. For every casting function $\delta$, there is a saturated casting function $\delta^{\prime}$ such that $K *_{\delta} \varphi=K *_{\delta^{\prime}} \varphi$.

Proof. Let us make $\delta^{\prime}(\varphi)=\operatorname{dec}(\bigcap \delta(\varphi))$. We need to show that $\delta^{\prime}(\varphi)=\operatorname{dec}\left(\bigcap \delta^{\prime}(\varphi)\right)$. Let $K^{\prime}$ be a theory. So, from Lemma $3.2, K^{\prime}=\bigcap \operatorname{dec}\left(K^{\prime}\right)$ which implies that

$$
\operatorname{dec}\left(K^{\prime}\right)=\operatorname{dec}\left(\bigcap \operatorname{dec}\left(K^{\prime}\right)\right)
$$

Let $K^{\prime}$ stand for $\bigcap \delta(\varphi)$. Thus, $\operatorname{dec}\left(K^{\prime}\right)=\operatorname{dec}(\bigcap \delta(\varphi))$ which means that

$$
\operatorname{dec}\left(K^{\prime}\right)=\delta^{\prime}(\varphi)
$$

Substitution of $\delta^{\prime}(\varphi)=\operatorname{dec}\left(K^{\prime}\right)$ in (B.10) gives us $\delta^{\prime}(\varphi)=\operatorname{dec}\left(\bigcap \delta^{\prime}(\varphi)\right)$.

Corollary B.1. Let $*_{\delta}$ be an ERF and $\varphi$ a formula. If $K *_{\delta} \varphi=\bigcap \delta(\varphi)$ and $\delta$ is saturated, then $\operatorname{dec}\left(K *_{\delta} \varphi\right)=\delta(\varphi)$

Proof. Let us suppose that $K *_{\delta} \varphi=\bigcap \delta(\varphi)$ and that $\delta$ is saturated. Then,

$$
\operatorname{dec}\left(K *_{\delta} \varphi\right)=\operatorname{dec}(\bigcap \delta(\varphi))
$$

Thus, as $\delta$ is saturated,

$$
\operatorname{dec}\left(K *_{\delta} \varphi\right)=\delta(\varphi)
$$

Lemma 3.8. Let $*_{\delta}$ be an ERF, $K$ a theory and $\varphi$ and $\psi$ two consistent formulae such that $K *_{\delta} \varphi=\bigcap \delta(\varphi)$ and $K *_{\delta} \varphi \wedge \psi=\bigcap \delta(\varphi \wedge \psi)$. If $\delta$ is saturated then

(R1) if $*_{\delta}$ satisfies $\left(\boldsymbol{K}_{7}^{*}\right)$ then $\delta(\varphi) \cap \omega(\psi) \subseteq \delta(\varphi \wedge \psi)$;

(R2) if $*_{\delta}$ satisfies $\left(\boldsymbol{K}_{8}^{*}\right)$ and $\delta(\varphi) \cap \omega(\psi) \neq \emptyset$ then $\delta(\varphi \wedge \psi) \subseteq \delta(\varphi) \cap \omega(\psi)$.

Proof. (R1) Let us suppose that $*_{\delta}$ satisfies $\left(\mathrm{K}_{7}^{*}\right)$. We sill show that $\delta(\varphi) \cap \omega(\psi) \subseteq \delta(\varphi \wedge \psi)$. Let $S$ be a consistent complete theory in $\delta(\varphi) \cap \omega(\psi)$, we only need to show that $S \in \delta(\varphi \wedge \psi)$. To show this, we will first need to show that $\bigcap(\varphi \wedge \psi) \subseteq S$. From $\left(\mathrm{K}_{7}^{*}\right)$, we get that $K *_{\delta} \varphi \wedge \psi \subseteq$ $K *_{\delta} \varphi+\psi$. From hypothesis, $K *_{\delta} \varphi=\bigcap \delta(\varphi)$ and $K *_{\delta} \varphi \wedge \psi=\bigcap \delta(\varphi \wedge \psi)$. Therefore,

$$
\bigcap \delta(\varphi \wedge \psi) \subseteq(\delta(\varphi))+\psi
$$


This implies that if a formula $\alpha \in \bigcap \delta(\varphi \wedge \psi)$, then $\alpha \in(\bigcap \delta(\varphi))+\psi$. Let $\alpha$ be a formula in $\bigcap \delta(\varphi \wedge \psi)$, then $\psi \rightarrow \alpha \in \bigcap \delta(\varphi)$. As $S \in \delta(\varphi)$, this give us that $\psi \rightarrow \alpha \in S$. Thus, as $\psi \in S$, we get that $\alpha \in S$. Therefore,

$$
\bigcap \delta(\varphi \wedge \psi) \subseteq S
$$

Now we are ready to show that $S \in \delta(\varphi \wedge \psi)$. As $K *_{\delta} \varphi \wedge \psi=\bigcap \delta(\varphi \wedge \psi)$, we get from the expression above that $K *_{\delta} \varphi \wedge \psi \subseteq S$. This implies that $S \in \operatorname{dec}\left(K *_{\delta} \varphi \wedge \psi\right)$. Moreover, from Corollary B.1 we have

$$
\delta(\varphi \wedge \psi)=\operatorname{dec}\left(K *_{\delta} \varphi \wedge \psi\right)
$$

Therefore, $S \in \delta(\varphi \wedge \psi)$.

(R2) Let us suppose that $*_{\delta}$ satisfies $\left(\mathrm{K}_{8}^{*}\right)$, and $\delta(\varphi) \cap \omega(\psi) \neq \emptyset$. Moreover, let $S$ a consistent complete theory in $\delta(\varphi \wedge \psi)$, we will show that $S \in \delta(\varphi) \cap \omega(\psi)$. To show this, we will first need to show that $\bigcap \delta(\varphi) \subseteq \bigcap \delta(\varphi \wedge \psi)$. Let $\alpha \in \bigcap \delta(\varphi)$. Thus, $\psi \rightarrow \alpha \in \bigcap \delta(\varphi)$ which implies that $\psi \rightarrow \alpha \in(\bigcap \delta(\varphi))+\psi$. As $K * \varphi \bigcap \delta(\varphi)$ and $\delta(\varphi) \cap \omega(\psi) \neq \emptyset$, we have that $\neg \psi \notin K * \varphi$. Thus, from $\left(\mathrm{K}_{8}^{*}\right)$, we get $\bigcap \delta(\varphi)+\psi \subseteq \bigcap \delta(\varphi \wedge \psi)$. This give us that $\psi \rightarrow \alpha \in \bigcap \delta(\varphi \wedge \psi)$. Thus, $\alpha \in \bigcap \delta(\varphi \wedge \psi)$. This means that

$$
\bigcap \delta(\varphi) \subseteq \bigcap \delta(\varphi \wedge \psi)
$$

As $S \in \delta(\varphi \wedge \psi)$, we have from (B.12) that $\bigcap \delta(\varphi) \subseteq S$. We have two cases to consider: either $\bigcap \delta(\varphi)=S$ or $\bigcap \delta(\varphi) \subset S$. The former case trivially implies that $\delta(\varphi)=\{S\}$. So we need to check only the latter case, that is, $\bigcap \delta(\varphi) \subset S$. This implies that $\bigcap \delta(\varphi) \cup S=S$. Let us fix $K=\bigcap \delta(\varphi)$. So, we have $K \subset S$ which implies that $K \cap \bigcap \delta(\varphi)=K$ and $K \cap S=K$. Thus, $K \cap S \subset S$. Putting it all together, we have that $K \cap \bigcap \delta(\varphi) \subseteq K \cap \bigcap\{S\}$, and $\bigcap \delta(\varphi) \cup \bigcap\{S\} \nsubseteq K$. Thus, from Lemma 2 (appendix to put), we get $\{S\} \subseteq \delta(\varphi)$ which means $S \in \delta(\varphi)$. As $\varphi \wedge \psi \in S$, we conclude that $S \in \omega(\psi)$ as well. Therefore, $S \in \delta(\varphi) \cap \omega(\psi)$.

Proposition 3.12. Let $*_{\delta}$ be an Exhaustive Revision Function that satisfies $\left(K_{7}^{*}\right)$ and $\left(K_{8}^{*}\right), \delta$ be saturated, and $<_{\delta}$ the projection of $\delta$. For every consistent formula $\varphi, \delta(\varphi)=\nu_{<\delta}(\varphi)$.

Proof. Let $\varphi$ be a consistent formula. As $\nu_{<\delta}(\varphi)=\max _{<\delta}(\omega(\varphi))$, we need to show that:

(i) if $A \in \max _{<}(\omega(\varphi))$, then $A \in \delta(\varphi)$

(ii) if $A \in \delta(\varphi)$, then $A \in \max _{<}(\omega(\varphi))$.

(i) Let us suppose for contradiction that there is a $A \in \max _{<}(\omega(\varphi))$ such that $A \notin \delta(\varphi)$. Therefore, it follows from the construction of $<$ that for some $B \in \delta(\varphi), A \leq B$ which implies that $A \notin \max _{<}(\omega(\varphi))$ which is a contradiction. 
(ii) $A \in \delta(\varphi)$. Let us suppose for contradiction that $A \in \delta(\varphi)$, but

$$
A \notin \max _{<\delta}(\omega(\varphi)) .
$$

Thus, for some $B \in \omega(\varphi),(A, B) \in<$.So we have two cases to consider: either (a) the pair $(A, B) \in<_{\varphi}$ or (b) the pair $(A<B)$ comes from a triangulation $\boldsymbol{\nabla}(\psi, \varphi)$ or $\boldsymbol{\nabla}(\varphi, \psi)$.

(a) $(A, B) \in<_{\varphi}$. Then, for some formula consistent formula $\psi, A, B \in \omega(\psi), B \in \delta(\psi)$ and $A \notin \delta(\psi)$. This implies that both $A$ and $B$ are in $\omega(\varphi) \cap \omega(\psi)$, which means that $A, B \in \omega(\varphi \wedge \psi)$. Moreover, as $*_{\delta}$ satisfies $\left(\mathrm{K}_{7}^{*}\right)$ and $\left(\mathrm{K}_{8}^{*}\right)$, it follows from $\mathrm{R} 1$ that $\delta(\varphi) \cap \omega(\psi) \subseteq \delta(\varphi \wedge \psi)$ and $\delta(\psi) \cap \omega(\varphi) \subseteq \delta(\varphi \wedge \psi)$ which implies that

$$
A \in \delta(\varphi \wedge \psi) \text { and } B \in \delta(\varphi \wedge \psi)
$$

Moreover, as $B \in \delta(\psi)$ and $B \in \omega(\varphi)$, we get that $\delta(\psi) \cap \omega(\varphi) \neq \emptyset$. Thus, we have from $\mathrm{R} 2$ that $\delta(\varphi \wedge \psi) \subseteq \delta(\psi) \cap \omega(\psi)$ which means that $A \in \delta(\psi)$ which is a contradiction.

(b) $(A, B) \in \mathbf{\nabla}(\psi, \varphi)$ or $(A, B) \in \mathbf{\nabla}(\varphi, \psi)$. The latter cases implies that $A \notin \delta(\varphi)$ which is a contradiction. So we are left with the former cases, that is, $(A, B) \in \mathbf{\nabla}(\psi, \varphi)$. Therefore, from triangulation definition we have that $A \in \omega(\psi) \backslash(\delta(\psi) \cup \delta(\varphi))$ and $B \in \delta(\varphi)$. This means that $A \notin \delta(\varphi)$ which is a contradiction, as from hypothesis $A \in \delta(\varphi)$.

Thus, we conclude that $A \in \max _{<\delta}(\omega(\varphi))$.

Theorem 3.14. Let $*_{\delta}$ be an ERF that satisfies both $\left(K_{7}^{*}\right)$ and $\left(K_{8}^{*}\right), \delta$ be saturated and let $<_{\delta}$ be the projection of $\delta$. The $C R F *_{<\delta}$ is equivalent to $*_{\delta}$.

Proof. Let $<_{\delta}$ be the projection of $\delta$. To show that $*_{\delta}$ and $*_{<\delta}$ are equivalent, we need to show that for every formula $\varphi, K *_{\delta} \varphi=K *_{<\delta} \varphi$. We split the proof in two cases: $\varphi$ is inconsistent or $\varphi$ is consistent.

(1) if $\varphi$ is inconsistent, then $K+\varphi=C n(\perp)$, which implies that $K *_{\delta} \varphi=C n(\perp)$ and $K *<\delta \varphi=C n(\perp)$. Thus,

$$
K *_{\delta} \varphi=K *_{<\delta} \varphi
$$

(2) $\varphi$ is consistent. There are two possible cases: $K+\varphi=L$ or $K+\varphi \neq L$. For the latter,

$$
K *_{\delta} \varphi=K *_{<\delta} \quad \varphi=K+\varphi .
$$

So we are left with the case $K+\varphi=L$, which implies that

$$
K *_{\delta} \varphi=\bigcap \delta(\varphi) \text { and } K *_{<\delta} \varphi=\bigcap \nu_{<\delta}(\varphi)
$$


As $\varphi$ is consistent, we have from Proposition 3.12 that $\delta(\varphi)=\nu_{<\delta}(\varphi)$. Therefore, $K *_{\delta} \varphi=$ $\bigcap \nu_{<\delta}(\varphi)$ which means that

$$
K *_{\delta} \varphi=K *_{<\delta} \varphi
$$

Therefore, we conclude that $*_{\delta}$ is equivalent to $*_{<\delta}$.

Corollary 3.7. Given a theory $K$ and an $E R F *_{\delta}$. If $*_{\delta}$ is $K$-royal and saturated then for every consistent formula $\varphi, K *_{\delta} \varphi=\bigcap \delta(\varphi)$.

Proof. It is straightforward from ERF definition. Let $\varphi$ be a consistent formula, such that $K+\varphi \neq$ $C n(\perp)$. We have two cases to consider:

(1) $K+\varphi=C n(\perp)$. Therefore, $K *_{\delta} \varphi=\bigcap \delta(\varphi)$.

(2) $K+\varphi \neq C n(\perp)$. Thus, $K *_{\delta} \varphi=K+\varphi$. Moreover, we have from Lemma 3.2 that $K+\varphi=\bigcap \operatorname{dec}(K+\varphi)$. Therefore,

$$
K *_{\delta} \varphi=\bigcap \operatorname{dec}(K+\varphi)
$$

As $\delta$ is $K$-royal, we have that $\delta(\varphi)=\operatorname{dec}(K+\varphi)$. Therefore,

$$
K *_{\delta} \varphi=\bigcap \delta(\varphi)
$$

Observation 3.12. Let $K$ be a theory, and $*_{\delta}$ an ERF, there is a saturated $K$-royal casting function $\delta^{\prime}$ such that $K *_{\delta} \varphi=K *_{\delta^{\prime}} \varphi$, for every formula $\varphi$.

Proof. We need to construct a $K$-royal casting function $\delta^{\prime}$. The first thing to do is to make $\delta(\varphi)=$ $\operatorname{dec}(K+\varphi)$, whenever $K+\varphi \neq C n(\perp)$. For the other cases, we just make $\delta^{\prime}$ mimick $\delta$. That is,

$$
\delta^{\prime}(\varphi)=\left\{\begin{array}{cl}
\operatorname{dec}(K+\varphi) & \text { if } K+\varphi \neq C n(\perp) \\
\delta(\varphi) & \text { otherwise. }
\end{array}\right.
$$

It is easy to see that $\delta^{\prime}$ is $K$-royal. The next step is to show that $*_{\delta}$ and $*_{\delta^{\prime}}$ are equivalent revision functions. Let $\varphi$ be a formula. If either $\varphi$ is inconsistent or $K+\varphi \neq C n(\perp)$, then $K *_{\delta} \varphi=K+\varphi$ and $K *_{\delta^{\prime}} \varphi=K+\varphi$. So we are left with the case that $K+\varphi=C n(\perp)$ and $\varphi$ is consistent. This means that

$$
K *_{\delta} \varphi=\bigcap \delta(\varphi) \text { and } K *_{\delta^{\prime}} \varphi=\bigcap \delta^{\prime}(\varphi)
$$


From the construction of $\delta^{\prime}$, we have that $\delta^{\prime}(\varphi)=\delta(\varphi)$. Therefore, $K *_{\delta} \varphi=\bigcap \delta^{\prime}(\varphi)$ which means that

$$
K *_{\delta} \varphi=K *_{\delta^{\prime}} \varphi
$$

Therefore, for every formula $\varphi, K *_{\delta} \varphi=K *_{\delta^{\prime}} \varphi$.

Lemma B.3. Let $*_{\delta}$ be an ERF revision function that satisfies ( $\left.K_{7}^{*}\right), K$ a theory and $\varphi$ a consistent formula. If $\delta$ is saturated and $K$-royal, and a complete theory $A$ belongs to $\omega(\varphi)$ but $A \notin \delta(\varphi)$, then $A \notin \delta(\varphi \vee \psi)$, for every formula $\psi$.

Proof. Let us suppose for contradiction that

$$
A \in \omega(\varphi), A \notin \delta(\varphi) \text {, but } A \in \delta(\varphi \vee \psi) \text {, for some formula } \psi \text {. }
$$

As $\varphi$ is consistent, so is $\varphi \vee \psi$. Therefore, as $\delta$ is $K$-royal, we have that $K *_{\delta} \varphi=\bigcap \delta(\varphi)$ and $K *_{\delta} \varphi \vee \psi=\bigcap \delta(\varphi \vee \psi)$. We recall that $(\varphi \vee \psi) \wedge \varphi \equiv \varphi$. Thus, we have from condition R1 of Lemma 3.8 that

$$
\begin{aligned}
& \delta((\varphi \vee \psi) \cap \omega(\varphi) \subseteq \delta((\varphi \vee \psi) \wedge \varphi) \\
& \delta((\varphi \vee \psi) \cap \omega(\varphi) \subseteq \delta(\varphi) .
\end{aligned}
$$

As $A \in \delta(\varphi \vee \psi)$, and $A \in \omega(\varphi)$, it follows from the expression above that $A \in \delta(\varphi)$ which is a contradiction. Therefore, we conclude that $A \notin \delta(\varphi \vee \psi)$.

Proposition B.1. Let $*_{\delta}$ be a fully AGM ration revision function, and $K$ and $\varphi$ be respectively $a$ theory and a consistent formula. If $\delta$ is saturated and $K$-royal and a complete theory $A$ belongs to $\omega(\varphi)$, but $A \notin \delta(\varphi)$ and $\delta(\varphi \vee \psi) \cap \omega(\psi) \neq \emptyset$ for some formula $\psi$, then $A \notin \delta(\psi)$.

Proof. Let us suppose for contradiction that $A \notin \delta(\varphi), \delta(\varphi \vee \psi) \cap \omega(\psi) \neq \emptyset$, but $A \in \delta(\psi)$. It follows from $\delta(\varphi \vee \psi) \cap \omega(\psi) \neq \emptyset$ that there is a complete theory that entails $\psi$. Therefore, both $\psi$ and $\varphi \vee \psi$ are consistent. Thus, as $\delta$ is $K$-royal we get that $K *_{\delta} \psi=\bigcap \delta(\psi)$ and $K *_{\delta} \varphi \vee \psi=\bigcap \delta(\varphi \vee \psi)$. This implies from R2 of Lemma 3.8 that

$$
\delta((\varphi \vee \psi) \wedge \psi) \subseteq \delta(\varphi \vee \psi) \cap \omega(\psi)
$$

As $(\varphi \vee \psi) \wedge \psi \equiv \psi$, we get

$$
\delta(\psi) \subseteq \delta(\varphi \vee \psi) \cap \omega(\psi)
$$

Thus, as $A \in \delta(\psi)$, we get that $A \in \delta(\varphi \vee \psi)$. Moreover, as $A \in \omega(\varphi)$ and $A \notin \delta(\varphi)$, we get from Lemma B.3 that $A \notin \delta(\varphi \vee \psi)$ which is a contradiction. Therefore, we conclude that $A \notin \delta(\psi)$.

Lemma B.4. Let $*_{\delta}$ be a fully AGM rational revision function, $K$ a theory and $\varphi$ a consistent theory. If $\delta$ is $K$-royal, and $M \in \delta(\varphi)$ and for some $M^{\prime} \in \omega(\varphi), M^{\prime} \in \delta(\varphi \vee \psi)$ then $M \in \delta(\varphi \vee \psi)$. 
Proof. Let us suppose for contradiction that $M \in \delta(\varphi)$ but $M \notin \delta(\varphi \vee \psi)$. As $M^{\prime} \in \delta(\varphi \vee \psi)$ and $M^{\prime} \in \omega(\varphi)$ we get that $\delta(\varphi \vee \psi) \cap \omega(\psi) \neq \emptyset$ which implies from condition R2 of Lemma 3.8 that

$$
\delta((\varphi \vee \psi) \wedge \varphi) \subseteq \delta(\varphi \vee \psi) \cap \omega(\varphi)
$$

As $(\varphi \vee \psi) \wedge \varphi) \equiv \varphi$, we have

$$
\delta(\varphi) \subseteq \delta(\varphi \vee \psi) \cap \omega(\varphi)
$$

As $M \in \delta(\varphi)$, the expression above implies that $M \in \delta(\varphi \vee \psi)$ which is a contradiction. Therefore, we conclude that $M \in \delta(\varphi \vee \psi)$.

Observation B.3. Let $\varphi$ and $\psi$ be two consistent formulae, and $<_{\delta}$ the projection of a casting function $\delta$ such that for some complete theories $A, B$ and $C: A \not \leq B, B \not \leq A, A \leq C$ but $B \not \leq C$. If $A \in \omega(\varphi) \backslash(\delta(\varphi) \cup \delta(\psi)$ and $C \in \delta(\psi)$, then $B \notin \omega(\varphi)$ and $B \notin \omega(\psi)$.

Proof. Let us suppose fro contradiction that $B \in \omega(\varphi)$ or $B \in \omega(\psi)$. Before we proceed, let us see what happens if $B \in \delta(\psi)$. As $A \in \omega(\varphi) \backslash(\delta(\varphi) \cup \delta(\psi)$, this would imply $(A, B) \in \mathbf{\nabla}(A, B)$ which means that $A \leq B$ which is a contradiction. Therefore,

$$
B \notin \delta(\psi)
$$

So, we proceed to the cases: $B \in \omega(\varphi)$ or $B \in \omega(\psi)$.

(1) $B \in \omega(\varphi)$. If $B \in \delta(\varphi)$ then we would have $A \leq B)$ which is a contradiction. If $B \notin \delta(\varphi)$, then as $B \in \delta(\psi)$, we get that $B \in \omega(\varphi) \backslash(\delta(\varphi \cup \delta(\psi))$ which implies that $(B, C) \in \mathbf{\nabla}(B, C)$ which means $B \leq C$ which is also a contradiction.

(2) $B \in \omega(\psi)$. However, as $B \notin \delta(\psi)$ it implies that $B \leq C$ which is a contradiction.

As both cases (1) and (2) lead to contradictions, we conclude that $B \notin \omega(\varphi)$ and $B \notin \omega(\psi)$.

Theorem 3.15. Let $K$ be a theory, and $*_{\delta}$ an an ERF that satisfies both ( $\left.K_{7}^{*}\right)$ and ( $\left.K_{8}^{*}\right)$. If $\delta$ is saturated and $K$-royal then its projection $<_{\delta}$ satisfies mirroring.

Proof. Let us suppose for contradiction that $<_{\delta}$ does not satisfies mirroring. Then, for some complete consistent theories $A, B$ and $C$ :

$$
A \not \leq B, B \not \leq A, A \leq C \text { but } B \not \subset C .
$$

As $\delta$ is $K$-royal, we get from Corollary 3.7 that

$$
\text { for every consistent theory } \varphi, K *_{\delta} \varphi=\bigcap \delta(\varphi) \text {. }
$$

As $A \leq C$, it follows from construction of $<$ that for some consistent formulae $\varphi$ and $\psi$ : either (i) $(A, C) \in<_{\varphi}$ or (ii) $(A, C) \in \mathbf{\nabla}(\varphi, \psi) \cup \mathbf{\nabla}(\psi, \varphi)$. 
(i) $(A, C) \in<_{\varphi}$. Therefore, $A \in \omega(\varphi)$ and $C \in \delta(\varphi)$. Note that $B$ is not at $\omega(\varphi)$. To see this, note that if $B$ were at $\omega(\varphi)$ then either $A \leq B$ (if $B \in \delta(\varphi)$ ) or $B \leq C$ (if $B \notin \delta(\varphi)$ ). Both cases leads to a contradiction. So, we get that

$$
B \notin \omega(\varphi) .
$$

As $B$ is a consistent complete theory, then $B$ entails some consistent formula $\alpha$. We have two cases to consider: $B \notin \delta(\alpha)$ or $B \in \delta(\alpha)$.

(1) $B \notin \delta(\alpha)$. Thus, as $B \in \omega(\alpha) \backslash \delta(\alpha)$ and $C \in \delta(\varphi)$, we get $(B, C) \in \mathbf{\nabla}(\alpha, \varphi)$. This means that $B \leq C$ which is a contradiction.

(2) $B \in \delta(\alpha)$. Let $M$ be a complete theory of $\delta(\varphi \vee \alpha)$. Thus, as $\delta(\varphi \vee \alpha) \subseteq \omega(\varphi \vee \alpha)$ and $\omega(\varphi \vee \alpha)=\omega(\varphi) \cup \omega(\alpha)$ : either $M \in \omega(\alpha)$ or $M \in \omega(\varphi)$.

(2a) $M \in \omega(\alpha)$. We get then from Lemma B.4 that $B \in \delta(\varphi \vee \alpha)$. As $\alpha$ is consistent and $\delta$ is $K$-royal, we have from Corollary 3.7 that $K *_{\delta} \alpha=\bigcap \delta(\alpha)$. Thus, as $B \in \omega(\alpha)$, we get $\delta(\varphi \vee \alpha) \cap \omega(\alpha) \neq \emptyset$. This implies from Proposition B.1 that $A \notin \delta(\alpha)$ which means that $(A, B) \in \mathbf{\nabla}(\varphi, \alpha)$. This means that $A \leq B$ which is a contradiction.

(2b) $M \in \omega(\varphi)$. Then, from Lemma B.4, $C \in \delta(\varphi \vee \alpha)$. We are left with two cases to consider regarding $B: B \in \delta(\varphi \vee \alpha)$ or $B \notin \delta(\varphi \vee \alpha)$. At case (2a) we already know that $B \in \delta(\varphi \vee \alpha)$ leads to a contradiction. So, we need to consider only the case $B \notin \delta(\varphi \vee \alpha)$ which gives us that $(B, C) \in<_{\varphi \vee \alpha}$. This implies that $B \leq C$ which is a contradiction.

Thus, we get that $(A, C) \notin<_{\varphi}$.

(ii) $(A, C) \in \mathbf{\nabla}(\varphi, \psi) \cup \mathbf{\nabla}(\psi, \varphi)$, that is, $(A, C) \in \mathbf{\nabla}(\varphi, \psi)$ or $(A, C) \in \mathbf{\nabla}(\varphi, \psi)$. Without loss of generality, let us assume that $(A, C) \in \mathbf{\nabla}(\varphi, \psi)$ (the another case is analogous and no further detail will be necessary). Therefore,

$$
A \in \omega(\varphi) \backslash(\delta(\varphi) \cup \delta(\psi)) \text {, and } C \in \delta(\psi) \text {. }
$$

From observation B.3, we have that

$$
B \notin \omega(\varphi) \text { and } B \notin \omega(\psi)
$$

As $B$ is a complete theory, we get that for some consistent formula $\alpha, B$ entails $\alpha$ which means that $B \in \omega(\alpha)$. We have two cases to consider: either $B \in \delta(\alpha)$ or $B \notin \delta(\alpha)$. In either case we will get a contradiction.

(3) For the case $B \notin \delta(\alpha)$, as $B \notin \omega(\psi)$, we get that $B \notin \delta(\psi)$. This implies that $B \notin \delta(\alpha) \cup \delta(\psi)$ which means that $B \in \omega(\alpha) \backslash(\delta(\alpha) \cup \delta(\psi))$. Hence, as $C \in \delta(\psi)$, we get that $(B, C) \in \mathbf{\nabla}(\alpha, \psi)$. This means that $B \leq C$ which is a contradiction. 
(4) For $B \in \delta(\alpha)$. Let $M$ be a complete theory of $\delta(\varphi \vee \alpha)$, which means that $M \in$ $\omega(\varphi) \cup \omega(\alpha)$. We have two cases to consider: $M \in \omega(\alpha)$ or $M \in \omega(\varphi)$.

(4a) $M \in \omega(\alpha)$. We get then from Lemma B.4 that $B \in \delta(\psi \vee \alpha)$. As $\alpha$ is consistent and $\delta$ is $K$-royal, we have from Corollary 3.7 that $K *_{\delta} \alpha=\bigcap \delta(\alpha)$. Thus, as $B \in \omega(\alpha)$, we get that $\delta(\varphi \vee \alpha) \cap \omega(\alpha) \neq \emptyset$. This implies from Proposition B.1 that $A \notin \delta(\alpha)$. Therefore, $A \in \omega(\varphi) \backslash(\delta(\varphi \cup \delta(\alpha))$ which means that $(A, B) \in \mathbf{\nabla}(\varphi, \alpha)$. This means that $A \leq B$ which is a contradiction.

(4b) $M \in \omega(\varphi)$. Then, from Lemma B.4, $C \in \delta(\psi \vee \alpha)$. We are left with two cases to consider: $B \in \delta(\psi \vee \alpha)$ or $B \notin \delta(\psi \vee \alpha)$. From case (4a), we already know that $B \in \delta(\psi \vee \alpha)$ leads to a contradiction. So, we need to check only the case $B \notin \delta(\psi \vee \alpha)$ which gives us that $(B, C) \in<_{\psi \vee \alpha}$. This implies that $B \leq C$ which is a contradiction.

As both (i) and (ii) leads to contradictions, we conclude then that $<_{\delta}$ satisfies mirroring.

Corollary 3.8. If an ERF $*_{\delta}$ satisfies both ( $\left.K_{7}^{*}\right)$ and $\left(K_{8}^{*}\right)$ and $\delta$ is saturated, then there is a CRV $*_{<}$equivalent to $*_{\delta}$ such that $<$ satisfies mirroring.

Proof. Let $K$ be a theory, and $*_{\delta}$ be an ERF that satisfies both $\left(\mathrm{K}_{7}^{*}\right)$ and $\left(\mathrm{K}_{8}^{*}\right)$. Then, from Observation 3.12 , there is a $K$-royal casting function $\delta^{\prime}$ such that the ERFs $*_{\delta}$ and $*_{\delta^{\prime}}$ are equivalent. This implies from Theorem 3.15 that the projection $<_{\delta^{\prime}}$ satisfies mirroring. Therefore, from Theorem 3.14 we get that the $\mathrm{CRF} *_{<\delta^{\prime}}$ is equivalent to $*_{\delta}$. 
APPENDIX B 


\section{Appendix C}

\section{Proofs for the Chapter 3, Section 3.5}

Theorem 3.17. If a contraction function $\dot{-}_{\delta}$ satisfies $\left(\boldsymbol{K}_{1}^{-}\right)$to $\left(\boldsymbol{K}_{4}^{-}\right)$and $\left(\boldsymbol{K}_{6}^{-}\right)$, then the revision operator $*$ obtained from $\dot{-}_{\delta}$ via the Levi's Identity satisfies the six AGM revision postulates.

Proof. $\left(\mathbf{K}_{1}^{*}\right)$ and $\left(\mathbf{K}_{2}^{*}\right)$ are trivial, and $\left(\mathbf{K}_{6}^{*}\right)$ is straightforward from $\left(\mathbf{K}_{6}^{-}\right)$.

$\left(\mathbf{K}_{3}^{*}\right) K * \varphi \subseteq K+\varphi$. From $\left(\mathbf{K}_{2}^{-}\right): K \dot{-}_{\delta} \neg \varphi \subset K$ or $K \dot{-}_{\delta} \neg \varphi=K$. As the latter case is trivial, we need to prove only the case $K \dot{-}_{\delta} \neg \varphi \subset K$. Therefore, from monotonicity, $\left(K \dot{-}_{\delta} \neg \varphi\right)+\varphi \subseteq K+\varphi$ which implies $K * \varphi \subseteq K+\varphi$.

$\left(\mathbf{K}_{4}^{*}\right) \neg \varphi \notin K$, then $K+\varphi \subseteq K * \varphi$. As $\neg \varphi \notin K$, it follows from $\left(\mathbf{K}_{3}^{-}\right)$that $K \dot{-}_{\delta} \neg \varphi=K$. Thus,

$$
\begin{aligned}
& K * \varphi=(K \dot{-} \delta \neg \varphi)+\varphi \\
& K * \varphi=K+\varphi
\end{aligned}
$$

which implies $K+\varphi \subseteq K * \varphi$.

$\left(\mathbf{K}_{5}^{*}\right) . K * \varphi=C n(\perp)$ iff $\neg \varphi \in C n(\emptyset)$. The direction $\Leftarrow$ is trivial, we need to prove only $\Rightarrow$ direction.

Let us suppose, for contradiction, that $K * \varphi=C n(\perp)$ and $\neg \varphi \notin C n(\emptyset)$. So, from $\left(\mathbf{K}_{4}^{-}\right)$, $\neg \varphi \notin K \dot{-}_{\delta} \neg \varphi$, which means that there is a model $M \in \llbracket K \dot{-}_{\delta} \neg \varphi \rrbracket$ such that $M \models \varphi$. This implies that $M \models\left(K \dot{-}_{\delta} \neg \varphi\right)+\varphi=K * \varphi$ which means $K * \varphi$ is consistent, that is, $K * \varphi \neq$ $C n(\perp)$ which contradicts our hypothesis. Therefore, we conclude that $\neg \varphi \in C n(\emptyset)$.

Theorem 3.18. If a contraction function $\dot{-}_{\delta}$ is fully AGM rational, then revision function * obtained via Levi identity is fully AGM rational.

Proof. ( $\mathbf{K}_{7}^{*}$ ) We need to show that $K * \varphi \wedge \psi \subseteq K * \varphi+\psi$. Let $\varphi \in K * \varphi \wedge \psi$, so we have to show that $\varphi \in K * \varphi+\psi$. From Levi's identity, we have

$$
K * \varphi \wedge \psi=K \dot{-}(\neg \varphi \vee \neg \psi)+\varphi \wedge \psi \text { and } K * \varphi=(K \dot{-} \neg \varphi)+\varphi
$$


First, notice that $(\neg \varphi \vee \neg \psi) \wedge(\varphi \rightarrow \psi) \equiv \neg \varphi$.

From $\left(\mathbf{K}_{7}^{-}\right)$, we have that $K \dot{-}-(\neg \varphi \vee \neg \psi) \cap K \dot{-}(\varphi \rightarrow \psi) \subseteq K \dot{-}(\neg \varphi \vee \neg \psi) \wedge(\varphi \rightarrow \psi)$, which implies that

$$
K \dot{-}(\neg \varphi \vee \neg \psi) \cap K \dot{-}(\varphi \rightarrow \psi) \subseteq K \dot{-} \neg \varphi .
$$

As $\alpha \in K * \varphi \wedge \psi=K \dot{-}(\neg \varphi \vee \neg \psi)+\varphi \wedge \psi$, it follows from Observation 5.2 that

$$
\varphi \wedge \psi \rightarrow \alpha \in K \dot{-}(\neg \varphi \vee \neg \psi)
$$

This means that $\varphi \wedge \psi \rightarrow \alpha \in K$, because $K \dot{-}(\neg \varphi \vee \neg \psi) \subseteq K$.

To make the things a bit clearer, let us fix $\beta=(\varphi \wedge \psi) \rightarrow \alpha$. So $\beta \in K$, which implies that $(\varphi \rightarrow \psi) \rightarrow \beta \in K$.

From $\left(\mathbf{K}_{5}^{-}\right), K \subseteq K \dot{-}(\varphi \rightarrow \psi)+\varphi \rightarrow \psi$. Therefore, as $\beta \in K$, we get $\beta \in K \dot{-}(\varphi \rightarrow \psi)+\varphi \rightarrow \psi$. Thus, from Observation 5.2,

$$
(\varphi \rightarrow \psi) \rightarrow \beta \in K \dot{-} \varphi \rightarrow \psi
$$

As $(\varphi \rightarrow \psi) \rightarrow \beta$ belongs to both $K \dot{-}(\neg \varphi \vee \neg \psi)$ and $K \dot{-} \varphi \rightarrow \psi$, it follows that

$$
(\varphi \rightarrow \psi) \rightarrow \beta \in K \dot{-} \neg \varphi
$$

This implies that $(\varphi \rightarrow \psi) \rightarrow \beta \in K \dot{-} \neg \varphi+\varphi$; that is, $(\varphi \rightarrow \psi) \rightarrow \beta \in K * \varphi$. Thus, $(\varphi \rightarrow \psi) \rightarrow \beta \in K * \varphi+\psi$.

As $\psi \in K * \varphi+\psi$, it follows that $\varphi \rightarrow \psi \in K * \varphi+\psi$. This together with identity above implies that $\beta \in K * \varphi+\psi$.

The final pieces of the puzzle are $\beta=(\varphi \wedge \psi) \rightarrow \alpha$ and $\varphi \wedge \psi \in K * \varphi+\psi$, which implies that $\alpha \in K * \varphi+\psi$, as we wanted to show.

( $\left.\mathbf{K}_{8}^{*}\right)$ Let us suppose that $\neg \psi \notin K * \varphi$. We want to show that $K * \varphi+\psi \subseteq K *(\varphi \wedge \psi)$. Let $\alpha \in K * \varphi+\psi$, we have to show that $\alpha \in K * \varphi \wedge \psi$. As $K * \varphi=K \dot{-} \neg \varphi+\varphi$ and $\neg \psi \notin K * \varphi$, it follows that

$$
\varphi \rightarrow \neg \psi \notin K \dot{-} \neg \varphi
$$

Now, note that $(\varphi \rightarrow \neg \psi) \wedge \neg \varphi \equiv \neg \varphi$. Thus, $\varphi \rightarrow \neg \psi \notin K \dot{-}(\varphi \rightarrow \neg \psi) \wedge \neg \varphi$. Thus, from $\left(\mathbf{K}_{8}^{-}\right)$,

$$
\begin{array}{r}
K \dot{-}(\varphi \rightarrow \neg \psi) \wedge \neg \varphi \subseteq K \dot{-} \varphi \rightarrow \neg \psi \\
K \dot{-} \neg \varphi \subseteq K \dot{-} \varphi \rightarrow \neg \psi
\end{array}
$$

As $\alpha \in K * \varphi+\psi$, it follows from Observation 5.2 that $\psi \rightarrow \alpha \in K * \varphi$. This implies that $\psi \rightarrow \alpha \in K \dot{-} \neg \varphi+\varphi$, because $K * \varphi=K \dot{-} \neg \varphi+\varphi$. Thus, it also follows from Observation 5.2 that

$$
\varphi \rightarrow(\psi \rightarrow \alpha) \in K \dot{-} \neg \varphi
$$


Thus from (C.5), $\varphi \rightarrow(\psi \rightarrow \alpha) \in K \dot{-}(\varphi \rightarrow \neg \psi)$. Thus, as $\varphi, \psi \in K \dot{-}(\varphi \rightarrow \neg \psi)+\varphi \wedge \psi=$ $K * \varphi \wedge \psi$, it follows that $\psi \rightarrow \alpha \in K * \varphi \wedge \psi$. Therefore, $\alpha \in K * \varphi \wedge \psi$.

Theorem 3.19. If a revision function $*$ is AGM rational, then the contraction function $\dot{-}_{\delta}$ obtained via Harper identity satisfies $\left(\boldsymbol{K}_{1}^{-}\right)$to $\left(\boldsymbol{K}_{6}^{-}\right)$.

Proof. Postulates $\left(\mathbf{K}_{1}^{-}\right)$and $\left(\mathbf{K}_{2}^{-}\right)$are trivial, and $\left(\mathbf{K}_{6}^{-}\right)$follows straightforwardly from $\left(\mathbf{K}_{6}^{*}\right)$.

$\left(\mathbf{K}_{3}^{-}\right)$Let us suppose that $\varphi \notin K$. Then, it follows from $\left(\mathbf{K}_{3}^{*}\right)$ and $\left(\mathbf{K}_{4}^{*}\right)$ that $K * \neg \varphi=K+\neg \varphi$. Thus,

$$
\begin{aligned}
K \dot{-}_{\delta} \varphi & =K \cap(K * \neg \varphi) \\
& =K \cap(K+\varphi) .
\end{aligned}
$$

As the logic is monotonic, we have that $K \subseteq K+\varphi$, which means that $K=K \cap(K+\varphi)$ which together with (C.6) implies

$$
K \dot{-}{ }_{\delta} \varphi=K
$$

$\left(\mathbf{K}_{4}^{-}\right)$There is nothing to prove for the case that $\varphi \equiv \perp$, as it is trivial. Then, we proceed with the proof for $\varphi$ as a consistent formula.

From $\left(\mathbf{K}_{2}^{*}\right)$, we have that $\neg \varphi \in K * \neg \varphi$ which together with $\left(\mathbf{K}_{5}^{*}\right)$ implies that $\varphi \notin K * \neg \varphi$. Thus, $\varphi \notin K * \neg \varphi$. This means that $\varphi \notin K \cap K * \neg \varphi$. Therefore, $\varphi \notin K \dot{-}_{\delta} \varphi$.

( $\left.\mathbf{K}_{5}^{-}\right)$Clearly, $K \subseteq K \cap(K * \neg \varphi)$ which from Harper Identity implies that

$$
K \subseteq K \dot{-}_{\delta} \varphi
$$

Moreover, as the logic is monotonic:

$$
K \dot{-}_{\delta} \varphi \subseteq\left(K \dot{-}_{\delta} \varphi\right)+\varphi
$$

Thus, from (C.7) and (C.8), we have

$$
K \subseteq\left(K \dot{-}_{\delta} \varphi\right)+\varphi
$$

Lemma 3.9. If $\varphi \notin K$, then $(K \cap K *(\neg \varphi \vee \neg \psi))+\neg \varphi \vee \neg \psi=K *(\neg \varphi \vee \neg \psi)$.

Proof. Let us suppose that $\varphi \notin K$. Thus, $\neg(\neg \varphi \vee \neg \psi) \equiv \varphi \wedge \psi \notin K$ which implies from (K $\left.\mathbf{K}_{3}^{*}\right)$ and $\left(\mathbf{K}_{4}^{*}\right)$ that $K *(\neg \varphi \vee \neg \psi)=K+(\neg \varphi \vee \neg \psi)$. Moreover, as $K \subseteq K+(\neg \varphi \vee \neg \psi)$, it follows that $K \cap K *(\neg \varphi \vee \neg \psi)=K$. Thus, 


$$
\begin{aligned}
(K \cap K *(\neg \varphi \vee \neg \psi))+(\neg \varphi \vee \neg \psi) & = \\
& =K+\neg \varphi \vee \neg \psi \\
& =K *(\neg \varphi \vee \neg \psi) .
\end{aligned}
$$

Theorem 3.20. If a revision function is fully AGM rational, then the contraction function obtained via Harper Identity is also fully AGM rational.

Proof. $\quad\left(\mathbf{K}_{7}^{-}\right)$We want to show that $K \dot{-} \varphi \cap K \dot{-} \psi \subseteq K \dot{-} \varphi \wedge \psi$. Let $\alpha \in K \dot{-} \varphi \cap K \dot{-} \psi$, then we need to show that $\alpha \in K \dot{-} \varphi \wedge \psi$. From harper identity,

$$
K-\varphi \wedge \psi=K \cap K *(\neg \varphi \vee \neg \psi)
$$

From $\left(\mathbf{K}_{7}^{*}\right)$ we have

$$
\begin{aligned}
& K *(\neg \varphi \vee \neg \psi) \wedge \neg \varphi \subseteq K *(\neg \varphi \vee \neg \psi)+\neg \varphi \\
& K *(\neg \varphi \vee \neg \psi) \wedge \neg \psi \subseteq K *(\neg \varphi \vee \neg \psi)+\neg \psi
\end{aligned}
$$

As $(\neg \varphi \vee \neg \psi) \wedge \neg \varphi \equiv \neg \varphi$ and $(\neg \varphi \vee \neg \psi) \wedge \neg \psi \equiv \neg \psi$,

$$
\begin{aligned}
& K * \neg \varphi \subseteq K *(\neg \varphi \vee \neg \psi)+\neg \varphi \\
& K * \neg \psi \subseteq K *(\neg \varphi \vee \neg \psi)+\neg \psi .
\end{aligned}
$$

As $\alpha \in K \dot{-} \varphi \cap K \dot{-} \psi$, we have that $\alpha \in K$ and also in both $K \dot{-} \neg \varphi$ and $K \dot{-} \neg \psi$. From Harper identity we have, $K \dot{-} \varphi=K \cap K * \neg \varphi$ and $K \dot{-} \psi=K \cap K * \neg \psi$. Thus, $\alpha \in K * \neg \varphi$ and $\alpha \in K * \neg \psi$. So, we have from (C.12) and (C.13) that

$$
\begin{aligned}
& \alpha \in K *(\neg \varphi \vee \neg \psi)+\neg \varphi, \text { and } \\
& \alpha \in K *(\neg \varphi \vee \neg \psi)+\neg \psi .
\end{aligned}
$$

Therefore, $\neg \varphi \rightarrow \alpha \in K *(\neg \varphi \vee \neg \psi)$ and $\neg \psi \rightarrow \alpha \in K *(\neg \varphi \vee \neg \psi)$, which implies that $(\neg \varphi \vee \neg \psi) \rightarrow \alpha \in K *(\neg \varphi \vee \neg \psi)$. Thus, as $\neg \varphi \vee \neg \psi \in K *(\neg \varphi \vee \neg \psi)$, we get $\alpha \in K *(\neg \varphi \vee \neg \psi)$. This means that $\alpha \in K \cap K * \neg \varphi \vee \neg \psi=K \dot{-} \varphi \wedge \psi$.

$\left(\mathbf{K}_{8}^{-}\right)$Let us suppose that $\varphi \notin K \dot{-}-(\varphi \wedge \psi)$. Let $\alpha \in K \dot{-}(\varphi \wedge \psi)$, we will show that $\alpha \in K \dot{-} \varphi$. From Harper Identity, we have that

$$
K \dot{-} \varphi \wedge \psi=K \cap(K * \neg \varphi \vee \neg \psi)
$$


As $\varphi \notin K \dot{-}(\varphi \wedge \psi)$, it follows from Observation 3.15 that

$$
\varphi \notin K \dot{-}(\varphi \wedge \psi)+(\neg \varphi \vee \neg \psi)
$$

By substituting, (C.14) in (C.15), we get that $\varphi \notin K \cap(K * \neg \varphi \vee \neg \psi)+\neg \varphi \vee \neg \psi$. This means that either $\varphi \notin K$ or $\varphi \notin K *(\neg \varphi \vee \neg \psi)$. The former implies, from Lemma 3.9 that $\varphi \notin K *(\neg \varphi \vee \neg \psi)$ as well. Thus, we have

$$
\varphi \notin K *(\neg \varphi \vee \neg \psi) .
$$

Thus from $\left(\mathrm{K}_{8}^{*}\right)$, we have that $K *(\neg \varphi \vee \neg \psi)+\neg \varphi \subseteq K *(\neg \varphi \vee \neg \psi) \wedge \neg \varphi$. As $(\neg \varphi \vee \neg \psi) \wedge \neg \varphi \equiv \neg \varphi$, we have that $K *(\neg \varphi \vee \neg \psi)+\neg \varphi \subseteq K * \neg \varphi$.

It follows from it that $\alpha \in K * \neg \varphi \cap K$, since from hypothesis $\alpha \in K \cap K *(\neg \varphi \vee \neg \psi)$. Therefore, as $K \cap K * \neg \varphi=K \dot{-} \varphi$, we have that

$$
\alpha \in K \dot{-} \varphi
$$


APPENDIX C 


\section{Appendix D}

\section{Proofs for Chapter 4}

Observation D.1. $\omega\left(K \cap K^{\prime}\right)=\omega(K) \cup \omega\left(K^{\prime}\right)$.

Proof. We have to show that

(i) $\omega(K) \cup \omega\left(K^{\prime}\right) \subseteq \omega\left(K \cap K^{\prime}\right)$;

(ii) $\omega\left(K \cap K^{\prime}\right) \subseteq \omega(K) \cup \omega\left(K^{\prime}\right)$.

Case (i) is easy to check. Let $S \in \omega(K) \cup \omega\left(K^{\prime}\right)$, then it either contains $K$ or $K^{\prime}$. In either case, since $K \cap K^{\prime}$ is a subset of both $K$ and $K^{\prime}$, we get that $K \cap K^{\prime} \subseteq S$. Therefore, $S \in \omega\left(K \cap K^{\prime}\right)$.

For case (ii), first notice that for any two collection of sets $A$ and $B$,

$$
\text { if } A \subseteq B \text {, then } \bigcap B \subseteq \bigcap A \text {. }
$$

Since we already proved case (i), we can use it in our behalf. From case (i) and the expression above we get that

$$
\bigcap\left(\omega(K) \cup \omega\left(K^{\prime}\right)\right) \subseteq \bigcap \omega\left(K \cap K^{\prime}\right)
$$

Another useful property of intersection we can use is that $\bigcap(A \cup B)=(\bigcap A) \cap(\bigcap B)$. This allows us to rewrite the expression above as follows:

$$
(\bigcap \omega(K)) \cap\left(\bigcap \omega\left(K^{\prime}\right)\right) \subseteq \bigcap \omega\left(K \cap K^{\prime}\right) .
$$

We recall that $\omega(S)=S$, for any consistent theory $S$. Therefore,

$$
K \cap K^{\prime} \subseteq K \cap K^{\prime}
$$

Theorem 4.2. Every splinter function $\diamond_{\delta}$ satisfies $(K \diamond 1)$ to $(K \diamond 5)$ and $(K \diamond 9)$.

Proof. Let $\diamond_{\delta}$ be a splinter function, and $K$ and $\varphi$ be respectively a theory and a formula. Postulates $(\mathrm{K} \diamond 1),(\mathrm{K} \diamond 2)$ and $(\mathrm{K} \diamond 4)$ are trivial. Postulate $(\mathrm{K} \diamond 5)$ is straightforward from condition (D4) of appointee definition. So, we are left with $(\mathrm{K} \diamond 3)$ and $(\mathrm{K} \diamond 9)$ : 
$(\mathbf{K} \diamond \mathbf{3})$ Let us suppose that $\varphi \in K$. If $K+\varphi=L$, then $K \diamond \varphi=L$ and $K=L$. Thus, $K=K \diamond \varphi$. On the other hand, if $K+\varphi \neq L$, then

$$
K \diamond \varphi=\bigcap_{S \in \omega(K)} \delta(S, \varphi)
$$

As $\varphi \in K$, we get that for every $S \in \omega(K), \varphi \in S$. Therefore, from condition (1) of $\delta$, we get that $\delta(S, \varphi)=\{S\}$. This means that

$$
\bigcap_{S \in \omega(K)} \delta(S, \varphi)=\bigcap \omega(K)
$$

Therefore, as $K=\bigcap \omega(K)$, we get that $K \diamond \varphi=K$.

$(\mathbf{K} \diamond \mathbf{9})$ Given two theories $K$ and $K^{\prime}$, we will show that

$$
\left(K \cap K^{\prime}\right) \diamond_{\delta} \varphi=\left(K \diamond_{\delta} \varphi\right) \cap\left(K \diamond_{\delta} \varphi\right)
$$

The case that either $\varphi$ is inconsistent or at least one of $K$ or $K^{\prime}$ is inconsistent is also trivial. So we proceed to the case that all of them are consistent. Thus,

$$
\begin{aligned}
K \diamond_{\delta} \varphi & =\bigcap_{S \in \omega(K)} \bigcap \delta(K, \varphi) \text { and } \\
K^{\prime} \diamond_{\delta} \varphi & =\bigcap_{S \in \omega\left(K^{\prime}\right)} \bigcap \delta(K, \varphi) .
\end{aligned}
$$

We will need the following property which is trivial to notice.

$$
\bigcap_{S \in(A \cup B)} S=\left(\bigcap_{S \in A} S\right) \cap\left(\bigcap_{S \in B} S\right)
$$

As $K$ and $K^{\prime}$ are consistent, we get that $K \cap K^{\prime}$ also is consistent. Therefore,

$$
\left(K \cap K^{\prime}\right) \diamond_{\delta} \varphi=\bigcap_{S \in \omega\left(K \cap K^{\prime}\right)} \bigcap \delta(S, \varphi) .
$$

From Observation D.1, $\omega\left(K \cap K^{\prime}\right)=\omega(K) \cup \omega\left(K^{\prime}\right)$. Thus, we can rewrite the expression above as

$$
\left(K \cap K^{\prime}\right) \diamond_{\delta} \varphi=\bigcap_{S \in \omega(K) \cup \omega\left(K^{\prime}\right)} \bigcap \delta(S, \varphi) .
$$


This together with (D.1), we get

$$
\begin{aligned}
& \left(K \cap K^{\prime}\right) \diamond_{\delta} \varphi= \\
& \quad\left(\bigcap_{S \in \omega(K)} \bigcap \delta(S, \varphi)\right) \cap\left(\bigcap_{S \in \omega\left(K^{\prime}\right)} \bigcap \delta(S, \varphi)\right) .
\end{aligned}
$$

Therefore,

$$
\left(K \cap K^{\prime}\right) \diamond_{\delta} \varphi=\left(K \diamond_{\delta} \varphi\right) \cap\left(K \diamond_{\delta} \varphi\right)
$$

Observation D.2. If a consistent theory $K$ is not complete, then $\omega(K)$ has at least two different complete consistent theories.

Proof. Let $K$ a consistent theory, but not complete. We need to show tha there are two different complete consistent treories $S_{1}$ and $S_{2}$ in $\omega(K)$. As $K$ is not complete, we get that for some formula $\varphi, K+\varphi \neq L$. Let $K_{1}=K+\varphi$ and $K_{2}=K+\neg \varphi$. Moreover, let $S_{1} \in \omega\left(K_{1}\right)$ and $S_{2} \in \omega\left(K_{2}\right)$. Clearly, $S_{1}$ and $S_{2}$ are different, since the former has the formula $\varphi$ and the latter $\neg \varphi$. Since $K$ is a subset of both of them, we get that $S_{1}$ and $S_{2}$ belongs to $\omega(K)$.

Lemma D.1. Let $\diamond$ be an update function, $K$ be a consistent theory and $\varphi$ a consistent formula. If for some $K^{\prime} \in \omega(K)$ a formula $\alpha$ is in $\bigcap_{S \in \omega(K)} S \diamond \varphi$ then $\alpha \in K^{\prime} \diamond \varphi$.

Proof. As $\alpha \in \bigcap_{S \in \omega(K)} S \diamond \varphi$, we get that $\alpha \in S \diamond \varphi$, for every $S \in \omega(K)$. As $K^{\prime} \in \omega(K)$, this implies that $\alpha \in K^{\prime} \diamond \varphi$.

Corollary D.1. Given a consistent theory $K$ and an update function $\diamond$. If $A \subseteq \omega(K)$ and a formula $\alpha$ belongs to $\bigcap_{S \in \omega(K)} S \diamond \varphi$ then $\alpha \in \bigcap_{S \in A} S \diamond \varphi$.

Proof. Let $\alpha \in \bigcap_{S \in \omega(K)} S \diamond \varphi$ and $K^{\prime} \in A$. Thus, $S \in \omega(K)$, as $A \subseteq \omega(K)$. Thus, from Lemma D.1, we get that $\alpha \in K^{\prime} \diamond \varphi$. This means that for all $S \in A, \alpha \in S \diamond \varphi$. Therefore, $\alpha \in \bigcap_{S \in A} S \diamond \varphi$.

Theorem 4.3. Every update function is a splinter

Proof. Given an $\diamond$ we will show that for some appointee $\delta$, the functions $\diamond$ and $\diamond_{\delta}$ coincide.

We construct $\delta$ as follows:

(i) $\delta(K, \varphi)=\omega(K \diamond \varphi)$, if both $K$ and $\varphi$ are consistent;

(ii) $\delta(K, \varphi)=T_{L}$, otherwise.

It is easy to check that $\delta$ is an appointee. Now we proceed to show that $\diamond_{\delta}$ and $\diamond$ coincides. First of all, we already have from Observation 4.1 that when either $K$ or $\varphi$ is inconsistent both $K \diamond \varphi$ and $K \diamond_{\delta} \varphi$ result in $C n(\perp)$. So, we are only left to the case that both $K$ and $\varphi$ are consistent. This means, we need to show that, for every consistent theory $K$ and consistent formula $\varphi$,

$$
K \diamond \varphi=\bigcap_{S \in \omega(K)} \bigcap \delta(S, \varphi)
$$


We recall that when $K$ is a complete consistent theory $\omega(K)=\{K\}$. Thus, $K \diamond_{\delta} \varphi=\bigcap \delta(K, \varphi)$. As $K$ is complete, we have from the construction of $\delta$, that $\delta(K, \varphi)=\omega(K \diamond \varphi)$. Therefore,

$$
\begin{aligned}
K \diamond_{\delta} \varphi & =\bigcap \delta(K, \varphi) \\
& =\bigcap \omega(K \diamond \varphi)
\end{aligned}
$$

As $\bigcap \omega(K \diamond \varphi)=K \diamond \varphi$, we have that for every complete consistent theory $K$,

$$
K \diamond_{\delta} \varphi=K \diamond \varphi
$$

This together with Observation 4.2 give us that

$$
K \diamond_{\delta} \varphi=\bigcap_{S \in \omega(K)} S \diamond \varphi
$$

To show that $\diamond$ and $\diamond_{\delta}$ match, we need to show that (a) $K \diamond \varphi \subseteq K \diamond_{\delta} \varphi$, and (b) $K \diamond_{\delta} \varphi \subseteq K \diamond \varphi$.

(a) We will show that $K \diamond \varphi \subseteq K \diamond_{\delta} \varphi$. It follows from the monotonicity of $\diamond$ that $K \diamond \varphi \subseteq S \diamond \varphi$, for every $S \in \omega(K)$. Thus,

$$
K \diamond \varphi \subseteq \bigcap_{S \in \omega(K)} S \diamond \varphi
$$

This implies from (D.2) that

$$
K \diamond \varphi \subseteq K \diamond_{\delta} \varphi
$$

(b) We will show that $K \diamond_{\delta} \varphi \subseteq K \diamond \varphi$.

We already have from (D.2) that this occurs when $K$ is a complete consistent theory. To conclude the proof, let us assume that $K$ is not complete. Let $\alpha \in K \diamond_{\delta} \varphi$, we will show that $\alpha \in K \diamond \varphi$. As $K$ is not complete, we have from Observation D.2, that $\omega(K)$ has at least two different complete consistent theories. Let $K_{1}$ be one of these theories in $\omega(K)$. Moreover, let us make $A=\omega(K) \backslash\left\{K^{\prime}\right\}$ which implies that $\omega(K)=A \cup\left\{K_{1}\right\}$. Hence,

$$
\bigcap\left(A \cup\left\{K_{1}\right\}\right)=\bigcap \omega(K) .
$$

We know that $A$ is not empty, since $\omega(K)$ has at least one other theory different from $K_{1}$. Moreover, recall that $\bigcap \omega(K)=K$. Thus, we get that

$$
K_{1} \cap \bigcap A=K .
$$

As $\diamond$ satisfies $(K \diamond 9)$, we get that

$$
\left(K_{1} \cap \bigcap A\right) \diamond \varphi=\left(K_{1} \diamond \varphi\right) \cap((\bigcap A \diamond \varphi)) .
$$


Therefore,

$$
K \diamond \varphi=\left(K_{1} \diamond \varphi\right) \cap((\bigcap A \diamond \varphi))
$$

In order to make things readable, let us make $K^{\prime}=\bigcap A$. We then rewrite the above formula as follows:

$$
K \diamond \varphi=\left(K_{1} \diamond \varphi\right) \cap\left(K^{\prime} \diamond \varphi\right)
$$

It is not hard to check that $\omega\left(K^{\prime}\right)=A$ which implies that $\omega\left(K^{\prime}\right) \subseteq \omega(K)$, as $A \subseteq \omega(K)$. As $\alpha \in K \diamond_{\delta} \varphi$, we get that $\alpha \in \bigcap_{S \in \omega(K)} S \diamond_{\delta} \varphi$. As $K_{1} \in \omega(K)$, it follows from Lemma D.1 that $\alpha \in K \diamond \varphi$. Moreover, as $\omega\left(K^{\prime}\right) \subseteq \omega(K)$, we get from Corollary D.1 that $\alpha \in \bigcap_{S \in \omega\left(K^{\prime}\right)} K \diamond \varphi$. Notice that $K \diamond \varphi=\bigcap_{S \in \omega\left(K^{\prime}\right)} K \diamond \varphi$. Therefore, $\alpha \in K \diamond \varphi$.

Thus, from (a) and (b) we conclude that $\diamond$ and $\diamond_{\delta}$ matches. Hence, we conclude that $\diamond$ is a splinter.

Observation D.3. Let $A$ be a set of complete consistent theories, and $K$ a theory. Let $\Gamma=\bigcap A$. Thus,

$$
\omega(C n(\Gamma \cup K))=\omega(\Gamma) \cap \omega(K)
$$

Proof. We will show that

$$
\omega(C n(\Gamma \cup K)) \subseteq \omega(\Gamma) \cap \omega(K) \text { and } \omega(\Gamma) \cap \omega(K) \subseteq \omega(C n(\Gamma \cup K)) .
$$

- $\omega(C n(\Gamma \cup K)) \subseteq \omega(\Gamma) \cap \omega(K)$. Let $S \in \omega(C n(\Gamma \cup K))$. Thus, $\Gamma \subseteq S$ and $K \subseteq S$. Therefore, $S \in \omega(\Gamma)$ and $S \in \omega(K)$.

- $\omega(\Gamma) \cap \omega(K) \subseteq \omega(C n(\Gamma \cup K))$. Let $S \in \omega(\Gamma) \cap \omega(K)$. Thus, $\Gamma \subseteq S$ and $K \subseteq S$. As $C n$ is Tarskian, this implies that $C n(\Gamma \cup K) \subseteq S$. Therefore, $S \in \omega(\Gamma \cup K)$.

Corollary D.2. Let $K_{1}, K_{2}$ and $K_{3}$ be three theories, if $K_{1} \subseteq C n\left(K_{2} \cup K_{3}\right)$, then $\omega\left(K_{2}\right) \cap \omega\left(K_{3}\right) \subseteq$ $\omega\left(K_{1}\right)$.

Proof. Let $K_{1} \subseteq C n\left(K_{2} \cup K_{3}\right)$, thus from Observation D.3, $\omega\left(K_{2} \cup K_{3}\right)=\omega\left(K_{2}\right) \cap \omega\left(K_{3}\right)$. Therefore,

$$
\omega\left(K_{1}\right) \subseteq \omega\left(K_{2}\right) \cap \omega\left(K_{3}\right)
$$

Corollary D.3. Given a theory $K, K+\varphi=\bigcap(\omega(K) \cap \omega(\varphi))$.

Proof. As $C n(K \cup C n(\varphi))=K+\varphi$, we get from Observation D.3,

$$
\omega(K+\varphi)=\omega(K) \cap \omega(\varphi)
$$


Therefore,

$$
\bigcap \omega(K+\varphi)=\bigcap(\omega(K) \cap \omega(\varphi)) .
$$

which implies

$$
K+\varphi=\bigcap(\omega(K) \cap \omega(\varphi)) .
$$

Proposition D.1. Let $\Gamma$ be a set of distinct complete theories and $B$ a consistent complete theory which is not in $\Gamma$. Then, $(B \cap \bigcap \Gamma) \subset \bigcap \Gamma$.

Proof. Analogous to the classical case.

Observation D.4. Let $A$ and $B$ be two collections of non-empty sets. If $A \subseteq B$ then $\bigcap B \subseteq \bigcap A$.

Proof. The case that $A=B$ is trivial. So we focus on $A \subset B$. Let us suppose for contradiction that $\bigcap B \nsubseteq \bigcap A$. Then, for some element $x \in \bigcap B, x \notin \bigcap A$. Thus,

$$
\text { for all } S \in B, x \in S \text { and }
$$

for some $S \in A, x \notin S$.

However, as $A \subseteq B$, it implies that every $S$ in $A$ is also in $B$. Let $S$ be one of the sets that does not have $x$. Therefore, $S \in B$ and $x \notin S$ which is a contradiction.

Observation D.5. Let $K$ be a theory, and $A$ be a set of a complete consistent theories. If $K=\bigcap A$ then $A=\omega(K)$.

Proof. As $\omega(K)$ have all the consistent complete theories that contains $K$, we get that $A \subseteq \omega(K)$. Let us suppose for contradiction that $A \neq \omega(K)$. Therefore

$$
A \subset \omega(K) .
$$

This implies that there is some complete consistent theory $S \in \omega(K)$ such that $S \notin A$. Thus, from Proposition D.1 that

$$
S \cap \bigcap A \subset \bigcap A .
$$

Notice that $A \subset A \cup\{S\}$ and $S \cap \bigcap A=\bigcap(A \cup\{S\})$. Therefore,

$$
\begin{aligned}
& \bigcap(A \cup\{S\}) \subset \bigcap A \\
& \bigcap(A \cup\{S\}) \subset K .
\end{aligned}
$$


So far, we have that $(A \cup\{S\}) \subseteq \omega(K)$ and $A \subset(A \cup\{S\})$. Thus, we get from Observation D.4 that

$$
\bigcap \omega(K) \subseteq \bigcap(A \cup\{S\}) \text { and } \bigcap(A \cup\{S\}) \subset \bigcap A \text {. }
$$

This means that, $\bigcap \omega(K) \subset \bigcap A$. Thus, $K \subset \bigcap A$ which is a contradiction. Therefore, $A=\omega(K)$.

Lemma 4.1. Let $\diamond_{\delta}$ be a royal splinter, and $K$ a complete consistent theory,

(i) if $\diamond_{\delta}$ satisfies $(K \diamond 6)$ then $\left.\delta(K, \varphi) \cap \omega(\psi) \subseteq \delta(K, \varphi \wedge \psi)\right)$;

(ii) if $\diamond_{\delta}$ satisfies $(K \diamond 8)$ then $\delta(K, \varphi) \cap \delta(K, \psi) \subseteq \delta(K, \varphi \vee \psi)$.

Proof. (i) From $(\mathrm{K} \diamond 6) K \diamond_{\delta} \varphi \wedge \psi \subseteq K \diamond_{\delta} \varphi+\psi$. Thus,

$$
\omega\left(K \diamond_{\delta} \varphi+\psi\right) \subseteq \omega\left(K \diamond_{\delta} \varphi \wedge \psi\right)
$$

As $\mathrm{K}$ is complete $K \diamond_{\delta} \varphi \wedge \psi=\bigcap \delta(K, \varphi \wedge \psi)$ which implies, from Observation D.5 that

$$
\omega\left(K \diamond_{\delta} \varphi \wedge \psi\right)=\delta(K, \varphi \wedge \psi)
$$

Therefore,

$$
\omega\left(K \diamond_{\delta} \varphi+\psi\right) \subseteq \delta(K, \varphi \wedge \psi)
$$

From Observation D.3 we get

$$
\omega\left(K \diamond_{\delta} \varphi+\psi\right)=\omega\left(K \diamond_{\delta} \varphi\right) \cap \omega(\psi)
$$

Thus,

$$
\omega\left(K \diamond_{\delta} \varphi\right) \cap \omega(\psi) \subseteq \delta(K, \varphi \wedge \psi)
$$

As $K$ is complete, we get that $K \diamond_{\delta} \varphi=\bigcap \delta(K, \varphi)$ which implies from Observation D.5 that $\omega\left(K \diamond_{\delta} \varphi\right)=\delta(K, \varphi)$. Therefore,

$$
\delta(K, \varphi) \cap \omega(\psi) \subseteq \delta(K, \varphi \wedge \psi)
$$

(ii) From $(\mathrm{K} \diamond 8) K \diamond_{\delta} \varphi \vee \psi \subseteq K \diamond_{\delta} \varphi \cup K \diamond_{\delta} \psi$. Thus,

$$
\omega\left(K \diamond_{\delta} \varphi \cup K \diamond_{\delta} \psi\right) \subseteq \omega(\varphi \vee \psi)
$$

From Observation D.3, we get

$$
\omega\left(K \diamond_{\delta} \varphi \cup K \diamond_{\delta} \psi\right)=\omega\left(K \diamond_{\delta} \varphi\right) \cap \omega\left(K \diamond_{\delta} \psi\right) .
$$


Thus,

$$
\omega\left(K \diamond_{\delta} \varphi\right) \cap \omega\left(K \diamond_{\delta} \psi\right) \subseteq \omega(\varphi \vee \psi)
$$

As $K$ is complete, we get $K \diamond_{\delta} \varphi=\bigcap \delta(K, \varphi), K \diamond_{\delta} \psi=\bigcap \delta(K, \psi)$ and $K \diamond_{\delta} \varphi \vee \psi=\bigcap \delta(K, \varphi \vee \psi)$. Therefore, from Observation D.5 we get that

$$
\begin{aligned}
\omega\left(K \diamond_{\delta} \varphi\right) & =\delta(K, \varphi) \\
\omega\left(K \diamond_{\delta} \psi\right) & =\delta(K, \psi) \\
\omega\left(K \diamond_{\delta} \varphi \vee \psi\right) & =\delta(K, \varphi \vee \psi) .
\end{aligned}
$$

Therefore,

$$
\delta(K, \varphi) \cap \delta(K, \psi) \subseteq \omega(\varphi, \psi) .
$$

Observation D.6. Let $\diamond_{\delta}$ be a royal splinter, and $K$ a complete consistent theory,

(i) $\delta(K, \varphi) \cap \omega(\psi) \subseteq \delta(K, \varphi \wedge \psi))$

(ii) $\delta(K, \varphi) \cap \delta(K, \psi) \subseteq \delta(K, \varphi \vee \psi)$.

Proof. (i) Let us suppose for contradiction that

$$
\delta(K, \varphi) \cap \omega(\psi) \not \delta(K, \varphi \wedge \psi)) .
$$

Thus, there exists some $S \in \delta(K, \varphi) \cap \omega(\psi)$ such that $S \notin \delta(K, \varphi \wedge \psi)$. Thus, for some $S^{\prime} \in \omega(\varphi \wedge \psi), S \leqslant S^{\prime}$ which implies that $S \notin \mu_{<}(\varphi)$. This means that $S \notin \delta(K, \varphi)$ which is a contradiction.

(ii) Let us suppose for contradiction that $\delta(K, \varphi) \cap \delta(K, \psi) \nsubseteq \delta(K, \varphi \vee \psi)$. Thus, there is some $S \in \delta(K, \varphi) \cap \delta(K, \psi)$ such that $S \notin \delta(S, \varphi \vee \psi)$. Thus, for some $S^{\prime} \in \omega(\varphi \vee \psi), S \leqslant S^{\prime}$. As $S^{\prime} \in \omega(\varphi \vee \psi)$, we have that $S^{\prime} \in \omega(\varphi)$ or $S^{\prime} \in \omega(\psi)$. In either case, we get that $S \notin \mu_{<}(\varphi)$ or $S \notin \mu_{<}(\psi)$. This means that $S \notin \delta(\varphi)$ or $S \notin \delta(\psi)$. Therefore, $S \notin \delta(\varphi) \cap \delta(\psi)$ which is a contradiction.

Theorem 4.4. Every royal splinter satisfies $(K \diamond 6)$ and $(K \diamond 8)$.

Proof. Let $\diamond_{\mu}$ be a royal splinter.

$(\mathbf{K} \diamond \mathbf{6})$ Let $K$ be a consistent theory. From Observation D.6 (i), we have that for every consistent complete theory $S$,

$\mu(S, \varphi) \cap \omega(\psi) \subseteq \mu(S, \varphi \wedge \psi))$. Therefore,

$$
\bigcap \mu(S, \varphi \wedge \psi) \subseteq \bigcap \mu(S, \varphi) \cap \omega(\psi)
$$


As $S$ is complete, we have that $S \diamond_{\mu} \varphi \wedge \psi=\bigcap \mu(S, \varphi \wedge \psi)$, and $S \diamond_{\mu} \varphi=\bigcap \mu(S, \varphi)$. Therefore, from Observation D.5, we have that $\omega\left(S \diamond_{\mu} \varphi\right)=\mu(S, \varphi)$. Therefore,

$$
S \diamond_{\mu} \varphi \wedge \psi \subseteq \bigcap \omega\left(S \diamond_{\mu} \varphi\right) \cap \omega(\psi)
$$

It follows from Corollary D.3 that $S \diamond_{\mu} \varphi+\psi=\bigcap \omega\left(S \diamond_{\mu} \varphi\right) \cap \omega(\psi)$. Therefore,

$$
S \diamond_{\mu} \varphi \wedge \psi \subseteq K+\psi
$$

As $K \subseteq S$, it follows from the monotonicity of $\diamond$ that

$$
K \diamond_{\mu} \varphi \wedge \psi \subseteq S \diamond_{\mu} \varphi \wedge \psi
$$

Therefore,

$$
K \diamond_{\mu} \varphi \wedge \psi \subseteq S \diamond_{\mu} \varphi+\psi, \text { for all } S \in \omega(K) .
$$

Recall that given a theory $K^{\prime}, K^{\prime}+\varphi=C n(K \cup C n(\varphi))$.

It follows from the expression above that,

$$
\begin{aligned}
K \diamond_{\mu} \varphi \wedge \psi & \subseteq \bigcap_{S \in \omega(K)}\left(S \diamond_{\mu} \varphi+\psi\right) \\
& \subseteq\left(\bigcap_{S \in \omega(K)} S \diamond_{\mu} \varphi\right)+\psi .
\end{aligned}
$$

As $S \diamond_{\mu} \varphi=\mu(S, \varphi)$, for every consistent complete theory $S$, we get that

$$
K \diamond_{\mu} \varphi \wedge \psi \subseteq\left(\bigcap_{S \in \omega(k)} \mu(S, \varphi)\right)+\psi
$$

Therefore, $K \diamond_{\mu} \varphi \wedge \psi \subseteq K \diamond_{\mu} \varphi+\psi$.

$(\mathbf{K} \diamond \mathbf{8})$ Let $K$ be a complete theory, so we have from Observation D.6 (ii) that $\mu(K, \varphi) \cap$ $\mu(K, \psi) \subseteq \mu(K, \varphi \vee \psi)$. This means that

$$
\bigcap(\mu(K, \varphi \vee \psi) \subseteq \bigcap(\mu(K, \varphi) \cap \mu(K, \psi))
$$

As $K$ is complete we get that $K \diamond_{\mu} \varphi=\bigcap \mu(K, \varphi)$ and $K \diamond_{\mu} \psi=\bigcap \mu(K, \psi)$. This implies, from Observation D.5 that $\omega\left(K \diamond_{\mu} \varphi\right)=\mu(K, \varphi)$ and $\omega\left(K \diamond_{\mu} \psi\right)=\mu(K, \psi)$. Thus,

$$
\bigcap\left(\mu(K, \varphi \vee \psi) \subseteq \bigcap\left(\omega\left(K \diamond_{\delta} \varphi\right) \cap \omega\left(K \diamond_{\delta} \psi\right)\right)\right.
$$

From Observation D.3, we have that $\omega\left(K \diamond_{\delta} \varphi \cup K \diamond_{\delta} \psi\right)=\omega\left(K \diamond_{\delta} \varphi\right) \cap \omega\left(K \diamond_{\delta} \psi\right)$. Therefore, 


$$
\bigcap\left(\mu(K, \varphi \vee \psi) \subseteq \bigcap\left(\omega\left(K \diamond_{\mu} \varphi \cup K \diamond_{\mu} \psi\right)\right)\right.
$$

this implies

$$
\bigcap\left(\mu(K, \varphi \vee \psi) \subseteq K \diamond_{\mu} \varphi \cup K \diamond_{\mu} \psi\right.
$$

As $K$ is complete we get that $K \diamond_{\mu} \varphi \vee \psi=\bigcap \mu(K, \varphi \vee \psi)$, which means

$$
K \diamond_{\mu} \varphi \vee \psi \subseteq K \diamond_{\mu} \varphi \cup K \diamond_{\delta} \psi
$$

Proposition 4.2. Let $\delta$ be the appointee from Example 16. The splinter $\diamond_{\delta}$ satisfies $(K \diamond 8)$ and $(K \diamond 6)$.

Proof. (1) Let us suppose for contradiction that $\diamond_{\delta}$ does not satisfy $(\mathrm{K} \diamond 8)$. Therefore, for some complete consistent theory $K$, there is some $A \in \delta(K, \varphi) \cap \delta(K, \psi)$ but $A \notin \delta(K, \varphi \vee \psi)$. Thus, $\delta(K, \varphi \vee \psi)=\omega(\varphi \vee \psi) \backslash \omega(G p)$. Moreover, If either $\delta(K, \varphi)=\omega(\varphi) \backslash G p$ or $\delta(K, \psi)=\omega(\psi) \backslash G p$ then $A \in \omega(\varphi \vee \psi) \backslash G p$. Therefore, we get that both $\delta(K, \varphi)=\omega(\varphi)$ and $\delta(K, \psi)=\omega(\psi)$. This means that $\omega(G p)$ is in both $\omega(\varphi)$ and $\omega(\psi)$ which implies that $G p \models \varphi \wedge \psi$ and $\varphi \models \bigvee S$ and $\psi \models \bigvee S$, for some finite set $S \subset \Gamma$. This implies that $\varphi \vee \psi \models \bigvee S$. Therefore, $\delta(K, \varphi \vee \psi)=\omega(\varphi)$ which is a contradiction.

(2) Let us suppose for contradiction that $\diamond_{\delta}$ does not satisfy $(\mathrm{K} \diamond 6)$. Thus, for some theory $K^{\prime}$, $K \diamond_{\delta} \varphi \wedge \psi \nsubseteq K \diamond_{\delta} \varphi+\psi$. This means that, for some complete consistent theory $K \in \omega(K)$, we get $\delta(K, \varphi) \cap \omega(\psi) \subseteq \delta(K, \varphi \wedge \psi)$. This implies, according to the definition of $\delta$, that $\delta(K, \varphi \wedge \psi)=$ $\omega(\varphi \wedge \psi) \backslash G p$ and $\delta(K, \varphi)=\omega(\varphi)$. This means that $G p \models \psi$ and $G p \models \varphi$ and $\varphi \models \bigvee S$, for some finite $S \subset \Gamma$. This implies that $G p \models \varphi \wedge \psi$ and $\varphi \wedge \psi \models \bigvee S$ which means that $\delta(K, \varphi \wedge \psi)=\omega(\varphi \wedge \psi)$ which is a contradiction.

Proposition 4.3. Every royal splinter satisfies DisCo.

Proof. Let us suppose for contradiction that not all royal splinter satisfies Disco. Thus, there is some royal splinter $\diamond_{\delta}$, a complete theory $K$, and a formula $\psi$ such that $K \diamond_{\delta} \psi \nsubseteq \bigcup_{\varphi \in \Gamma} K \diamond_{\delta} \varphi$, for some set of formulae $\Gamma$. As $\diamond_{\delta}$ is royal, we have that $\delta$ is relational. Let $<$ be the relation over which $\delta$ relies to choose its complete theories. So we get that

$$
\bigcap_{\varphi \in \Gamma} \delta(K, \varphi) \not \subset \delta(K, \psi)
$$

This means that there is some complete theory $A$ in $\delta(K, \varphi)$, for every $\varphi \in \Gamma$, but $A \notin \delta(K, \psi)$. Therefore, $A$ is not maximal which implies that there is some complete theory $B \in \omega(\psi)$ such that $(A, B) \in<$. As $C n(\psi)=\bigcap_{\varphi \in \Gamma} C n(\varphi)$, we get that $\omega(\psi)=\bigcup_{\varphi \in \Gamma} \omega(\varphi)$. Therefore, $B \in \omega(\varphi)$ for 
some $\varphi \in \Gamma$. This implies that $A$ is not maximal in $\omega(\varphi)$ modulo $<$. This implies that $A \notin \delta(K, \varphi)$ which is a contradiction.

Lemma 4.3. Let $\diamond_{\mu}$ be a royal splinter, such that $\mu$ is quasi-reflected. Given a complete consistent theory $K$, if $\varphi \in K \diamond_{\mu} \psi$ and $\psi \in K \diamond_{\mu} \varphi$, then $K \diamond_{\mu} \varphi=K \diamond_{\mu} \psi$.

Proof. Let be an splinter. Let us suppose, for contradiction, that $\mu$ is reflected, but $\diamond_{\mu}$ does not satisfies $(\mathrm{K} \diamond 7)$. Therefore, for some theory $K$ and formulae $\varphi$ and $\psi$, we have that $\psi \in K \diamond_{\mu} \varphi$ and $\varphi \in K \diamond_{\mu} \psi$, but $K \diamond_{\mu} \varphi \neq K \diamond_{\mu} \psi$. Therefore, either $K \diamond_{\mu} \varphi \nsubseteq K \diamond_{\mu} \psi$, or $K \diamond_{\mu} \varphi \nsubseteq K \diamond_{\mu} \psi$. Without loss of generality, let us assume that

$$
K \diamond_{\mu} \varphi \nsubseteq K \diamond_{\mu} \psi
$$

As $K \diamond_{\mu} \varphi=\bigcap \mu(K, \varphi)$ and $K \diamond_{\mu} \psi=\bigcap \mu(K, \psi)$. Therefore,

$$
\bigcap \mu(K, \varphi) \nsubseteq \bigcap \mu(K, \psi) \text {. }
$$

Notice that for any two collection of sets $A$ and $B$,

$$
\text { if } A \subseteq B \text {, then } \bigcap B \subseteq \bigcap A \text {. }
$$

Therefore, from the counterpositive of the expression above, we get that $\mu(K, \psi) \nsubseteq \mu(K, \varphi)$. Therefore, there is some consistent complete theory $S \in \mu(K, \psi)$ such that

$$
S \notin \mu(K, \varphi) .
$$

Thus, $S \notin \max _{\leqslant K}\left(\omega(\varphi)\right.$ which means that $S \leqslant_{K} S^{\prime}$, for some complete theory $S^{\prime} \in \omega(\varphi)$. As $\varphi \in K \diamond_{\mu} \psi$, we get that $\psi \in S$ which means that $S \in \omega(\psi)$. Therefore, as $S \in \diamond_{\mu}(K, \psi)$, we get that $S^{\prime} \notin \omega(\psi)$.

Moreover, let $B \in \mu(K, \psi)$. As $\varphi \in K \diamond_{\mu} \psi$, we get that $B \in \omega(\varphi)$. Therefore, as both $S$ and $B$ are in $\omega(\varphi) \cap \omega(\psi)$ and $S \in \mu(K, \varphi)$ and $B \in \mu(K, \psi)$, we get that $S \Varangle_{K} S$ and $S \Varangle_{K} B$. Thus, as $S \leqslant_{K} S^{\prime}$ and $\leqslant_{K}$ satisfies quasi-reflection, we get thet either (i) $S \leqslant_{K} S^{\prime}$ or (ii) if $S^{\prime} \leqslant_{K} C$ then $B \leqslant_{K} C$. The first is not possible, since we know that $B \in \mu(K, \psi)$, that is, $B \Varangle_{K} S^{\prime}$. Thus, we focus only on the second case:

$$
\text { if } S^{\prime} \leqslant_{K} C \text { then } B \leqslant{ }_{K} C \text {. }
$$

As $\varphi \in K \diamond_{\mu} \psi$, it follows that every theory $A \in \delta(K, \psi)$ satisfies $\varphi$. This means that if $A \in$ $\mu(K, \psi)$, then $A \in \omega(\varphi)$. As $S^{\prime} \notin \omega(\varphi)$, we get that $S^{\prime} \notin \diamond_{\mu}(K, \psi)$. Therefore, $S^{\prime} \leqslant C$, for some $C \in$ $\omega(\psi)$. Therefore, we have from the expression above that $B \leqslant{ }_{K} C$ which implies that $B \notin \mu(K, \psi)$. This means $B \notin \delta(K, \psi)$ which is a contradiction. Thus, we conclude that $K \diamond_{\mu} \varphi=K \diamond_{\mu} \psi$.

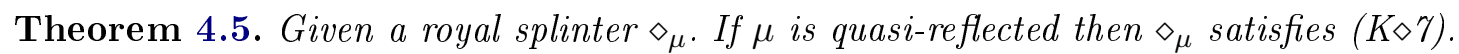

Proof. Let $K$ be a theory, and $\diamond_{\mu}$ an splinter function whose appointee $\mu$ is quasi-reflected. 
Moreover, let $\varphi$ and $\psi$ be two formulae such that $\varphi \in K \diamond_{\mu} \psi$ and $\varphi \in K \diamond_{\mu} \psi$. We need to show that $K \diamond_{\mu} \varphi=K \diamond_{\mu} \psi$. We have to cases to consider, (i) $K \diamond_{\mu} \varphi=C n(\perp)$ or $K+\varphi \neq C n(\perp)$.

(i) $K+\varphi=C n(\perp)$. Then, either $K=C n(\perp)$ or $C n(\varphi)=C n(\perp)$. From the former $K \diamond_{\mu} \psi=$ $C n(\perp)$. For the latter, we get that $\perp \in K \diamond_{\mu} \psi$ which implies that $K \diamond \psi=C n(\perp)$. Therefore, in either case, $K \diamond_{\mu} \varphi=K \diamond_{\mu} \psi$.

(ii) $K+\varphi \neq C n(\perp)$. Thus, $K \neq C n(\perp)$, and every formula in $K \diamond_{\mu} \varphi$ is consistent. As $\psi \in K \diamond_{\mu} \varphi$ we get $\psi$ is consistent. Therefore, $K \diamond_{\mu} \psi \neq C n(\perp)$. Thus, we have that,

$$
\begin{aligned}
K \diamond_{\mu} \varphi & =\bigcap_{S \in \omega(K)} \delta(S, \varphi) \text { and } \\
K \diamond_{\mu} \psi & =\bigcap_{S \in \omega(K)} \delta(S, \psi) .
\end{aligned}
$$

Let $S$ be a complete consistent theory that contains $K$, that is, $K \subseteq S$. Thus,

$$
K \diamond_{\mu} \varphi \subseteq S \diamond_{\mu} \varphi \text { and } K \diamond_{\mu} \diamond \psi \subseteq S \diamond_{\mu} \psi
$$

As $\psi \in K \diamond_{\mu} \varphi$, we get that $\psi \in S \diamond_{\mu} \varphi$. Analogously, $\varphi \in K \diamond_{\mu} \psi$. Therefore, from Lemma 4.3 we get that $S \diamond_{\mu} \varphi=S \diamond_{\mu} \psi$. So we get that,

$$
K \diamond_{\mu} \varphi=\bigcap_{S \in \omega(K)} S \diamond_{\mu} \varphi
$$

Which implies that

$$
K \diamond_{\mu} \varphi=\bigcap_{S \in \omega(K)} S \diamond_{\mu} \psi
$$

Therefore, as $S \diamond_{\mu} \psi=\bigcap \delta(S, \psi)$, we get that

$$
K \diamond_{\mu} \varphi=\bigcap_{S \in \omega(K)} \delta(S, \psi)
$$

Thus,

$$
K \diamond_{\mu} \varphi=K \diamond_{\mu} \psi
$$

Proposition 4.4. Given a splinter $\diamond_{\delta}$, and a consistent complete theory $K$. If $\diamond_{\delta}$ is fully $K M$ rational, then the projection of $\delta$ modulo $K$ is the silhouette of $\delta$ within $K$.

Proof. Let $K$ be a consistent complete theory, and $\leqslant_{K}$ the projection of $\delta$ modulo $K$. To show that $\leqslant_{K}$ is the silhouette of $\delta$ modulo $K$, we need to show that for every formula consistent formula $\varphi$

$$
\delta(K, \varphi)=\operatorname{Max}_{\leqslant_{K}}(\omega(\varphi)) .
$$


We need to show that (i) $\delta(\varphi) \subseteq \operatorname{Max}_{\leqslant_{K}}(\varphi)$ and (ii) $\operatorname{Max}_{\leqslant_{K}}(\varphi) \subseteq \delta(\varphi)$.

(i) $\delta(K, \varphi) \subseteq \operatorname{Max}_{\leqslant_{K}}(\varphi)$. Let us suppose for contradiction that $\delta(K, \varphi) \nsubseteq \operatorname{Max}_{\leqslant_{K}}(\varphi)$.

Thus, there is a complete consistent theory $A \in \delta(K, \varphi)$ such that $A \notin \operatorname{Max}_{\leqslant_{K}}(\varphi)$. Therefore, there is some $B \in \omega(\varphi)$ such that $(A, B) \in \leqslant_{K}$ but $(B, A) \notin \leqslant K$. From $\leqslant_{K}$ definition, it means that for some formula $\psi,(A, B) \in \leqslant_{\psi}$ and

$$
(B, A) \notin \leqslant \alpha^{\prime}, \text { for all formula } \alpha^{\prime} .
$$

This means that for all formula $\alpha^{\prime}$,

$$
B \in \delta\left(K, \alpha^{\prime}\right) \text { or both } A \text { and } B \text { are in } \delta(K, \alpha), \text { for some formula } \alpha \text {. }
$$

From $(A, B) \in \leqslant_{\psi}$ we get $A \notin \delta(\psi)$ and

either $A$ or $B$ are not in $\delta(K, \alpha)$, for all formula $\alpha$.

As $A$ is in both $\omega(\psi)$ and $\delta(K, \varphi)$, we have from Lemma 4.1 that $A \in \delta(K, \varphi \wedge \psi)$. Thus, from (D.5) above we get $B \notin \delta(K, \varphi \wedge \psi)$. This implies from the counterpositive of Lemma 4.1 (i) that $B \notin \delta(K, \psi)$. This implies from (D.4) above that for some formula $\alpha$, both $A$ and $B$ are in $\delta(K, \alpha)$ which contradicts (D.5). Thus, we conclude that $\delta(K, \varphi) \subseteq \operatorname{Max}_{\leqslant_{K}}(\varphi)$.

(ii) $\operatorname{Max}_{\leqslant_{K}}(\varphi) \subseteq \delta(K, \varphi)$. Let us suppose for contradiction that $\operatorname{Max}_{\leqslant_{K}}(\varphi) \nsubseteq \subseteq \delta(K, \varphi)$. This means that there is a complete consistent theory $A \in \max _{<}(\varphi)$ such that $A \notin \delta(\varphi)$. Therefore, for every $B \in \omega(\varphi),(A, B) \notin \leqslant_{\varphi}$. This implies, from $\leqslant_{\varphi}$ definition that either $A \in \delta(\varphi)$ or there is some formula $\psi$, such that both $A$ and $B$ are in $\delta(\psi)$. As $A \notin \delta(\varphi)$, we get that

for all $B \in(\omega(\varphi) \backslash\{A\}), A$ and $B$ are in $\delta(\psi)$ for some $\psi$.

Let us then assign for each complete consistent theory $B$ a formula $\psi_{B}$ such that $A, B \in \delta\left(\psi_{B}\right)$. Moreover, let $\Gamma=\left\{\psi_{B} \mid B \in \omega(\varphi) \backslash\{A\}\right\}$ be the collection of all these selected formulae. Note that $B \in \psi_{B} \wedge \varphi$, as $B \in \omega\left(\psi_{B}\right)$ and $\omega(\varphi)$. Moreover, from Lemma 4.1, we get $A \in \delta\left(\psi_{B} \wedge \varphi\right)$, since $A \in \delta\left(\psi_{B}\right) \cap \omega(\varphi)$. In summary,

$$
B \in \omega\left(\psi_{B} \wedge \varphi\right) \text { and } A \in \delta\left(\psi_{B} \wedge \varphi\right), \text { for every } \psi_{B} \in \Gamma
$$

So, let us make $\Gamma_{\wedge}=\left\{\psi_{B} \wedge \varphi \mid \psi_{B} \in \Gamma\right\}$. So, we get

$$
\omega(\varphi)=\bigcup_{\alpha \in \Gamma_{\wedge}} \omega(\alpha)
$$

Thus,

$$
\bigcap_{\alpha \in \Gamma_{\wedge}} \delta(\alpha) \subseteq \delta(\varphi) .
$$


Therefore, as for each $\alpha \in \Gamma_{\wedge}, \alpha=\psi_{B} \wedge \varphi$, and $A \in \delta\left(K, \psi_{B} \wedge \varphi\right)$ we get that

$$
A \in \bigcap_{\alpha \in \Gamma_{\wedge}} \delta(\alpha)
$$

which means that $A \in \delta(\varphi)$ which is a contradiction.

Theorem 4.6. The appointee of a fully KM rational function $\diamond_{\delta}$ is relational.

Proof. Let $\diamond_{\delta}$ be a fuly KM rational function, therefore a splinter. To show that $\delta$ is relational, we need to show that for every complete consistent theory $S$, there is a contra-headed relation $\leqslant_{S}$ such that $\delta(S, \varphi)=\operatorname{Max}_{\leqslant_{S}}(\omega(\varphi))$. In other words, we need to construct a silhouette $\leqslant_{S}$ of $\delta$ within $S$. From Proposition 4.4, we get that the projection of $\delta$ modulo $S$ is the silhouette $\leqslant_{S}$ of $\delta$ within $S$. This also implies that $\leqslant_{S}$ is a contra-headed. To see this, notice that for every consistent formula $\varphi, \operatorname{Max}_{\leqslant S}(\omega(\varphi))=\delta(S, \varphi)$. Moreover, as $\delta$ is an appointee, it follows from condition (D1) of appointee definition that $\delta(S, \varphi) \neq \emptyset$ whenever $\varphi$ is consistent. Therefore, $\operatorname{Max}_{\leqslant S}(\omega(\varphi)) \neq \emptyset$, for every consistent formula $\varphi$, that is, $\leqslant_{S}$ satisfies maximal-cut. This means that $\leqslant_{S}$ is contraheaded.

Theorem 4.7. Let $\mu$ be the appointee from a fully KM rational splinter. Given a complete consistent theory $K$, the projection $\leqslant_{K}$ of $\delta$ modulo $K$ is quasi-reflected.

Proof. Let $\diamond_{\mu}$ be a fully rational splinter, and $S$ a consistent complete theory. Moreover, let $\leqslant S$ be the projection of $\mu$ modulo $S$. We will show that $\leqslant_{S}$ is quasi-reflected. Let us suppose for contradiction that it is not quasi-reflected. Then, for some complete consistent theories $A, B$ and $C$ :

$$
A \Varangle_{S} B, B \Varangle_{S} A, A \leqslant{ }_{S} C \text {, but } B \nless_{S} C
$$

As $A$ is a consistent complete theory, it follows that for some consistent formula $\varphi, A \in \omega(\varphi)$. This gives us two main cases to consider: (1) $B \in \omega(\varphi)$ or (2) $B \notin \omega(\varphi)$.

(1) $B \in \omega(\varphi)$. As $A \leqslant_{S} C$, we get that: (a) the pair $(A, C)$ is either in $\leqslant_{\varphi}$ or (b) for some formula $\alpha,(A, C) \in \nabla(\varphi, \alpha)$ or $(A, C) \in \nabla(\alpha, \varphi)$.

(a) $(A, C) \in \leqslant_{\varphi}$. This implies that $C \in \delta(\varphi)$, and $A \notin \delta(\varphi)$. Concerning $B$, we have that either $B \in \delta(\varphi)$, which implies that $A \leqslant_{\varphi} B$, or $B \notin \delta(\varphi)$, which leads to $B \leqslant_{\varphi} C$. In either case we reach a contradiction, as $A \Varangle_{S} B$ and $B \Varangle_{S} C$.

(b) $(A, C) \in \nabla(\varphi, \alpha)$ or $(A, C) \in \nabla(\alpha, \varphi)$. For the latter case, we have that $A \notin \omega(\varphi)$ which is a contradiction. So we focus only on the case $(A, C) \in \nabla(\varphi, \alpha)$. So $A \notin \delta(\varphi)$ and $C \in \delta(\alpha)$. Regarding $B$, we have that either $B \in \delta(\varphi)$, which implies that $A \leqslant S B$, or $B \notin \delta(\varphi)$ which implies that $B \leqslant_{S} C$. In either case we get a contradiction, as $A \Varangle_{S} B$ and $B \&_{S} C$.

(2) $B \notin \omega(\varphi)$. Thus, for some formula $\psi, B \in \omega(\psi)$. Moreover, for some formula $\alpha$ : either (a) $(A, C) \in \leqslant_{\alpha}$ or (b) $(A, C) \in \nabla(\varphi, \alpha)$ or $(A, C) \in \nabla(\alpha, \varphi)$. 
(a) $(A, C) \in \leqslant \alpha$. This means that $A \notin \delta(\alpha)$ and $C \in \delta(\alpha)$. Regarding $B$, we have two cases: (i) $B \in \omega(\alpha)$ or (ii) $B \notin \omega(\alpha)$.

(i) $B \in \omega(\alpha)$. Thus, either $B \in \delta(\alpha)$ which implies that $A \leqslant S B$, or $B \notin \delta(\alpha)$ which implies that $B \leqslant_{S} C$. In either case, we reach a contradiction, as $A \Varangle_{S} B$ and $B \Varangle_{S} C$.

(ii) $B \notin \omega(\alpha)$. Thus, either $B \in \delta(\varphi)$, which implies from the triangulation $\nabla(\alpha, \psi)$ that $A \leqslant S B$, or $B \notin \delta(\psi)$ which implies from the triangulation $\nabla(\psi, \alpha)$ that $B \leqslant{ }_{S} C$. In ither case we get a contradiction, since $A \Varangle_{S} B$ and $B \Varangle_{S} C$.

(b) $(A, C) \in \nabla(\varphi, \alpha)$ or $(A, C) \in \nabla(\alpha, \varphi)$. For the latter case, we have that $A \notin \omega(\varphi)$ which is a contradiction. So we proceed to the case $(A, C) \in \nabla(\varphi, \alpha)$. Thus, $A \notin \delta(\varphi)$ and $C \in \delta(\alpha)$. Regarding $B$, we have that: either (i) $B \in \delta(\psi)$ or (ii) $B \notin \delta(\psi)$.

(i) $B \in \delta(\psi)$. Thus, from the triangulation $\nabla(\varphi, \psi)$ we have that $A \leqslant_{S} B$, which is a contradiction, since $A \$_{S} B$.

(ii) $B \in \delta(\psi)$. In the case that $B \notin \omega(\alpha)$ we get from $\nabla(\psi, \alpha)$ that $B \leqslant{ }_{S} C$ which is a contradiction. On the other hand, if $B \in \omega(\alpha)$ we get that either $B \in \delta(\alpha)$, which implies that $A \leqslant S B$, or $B \notin \delta(\alpha)$ which implies that $B \leqslant{ }_{S} C$. In either case, we get a contradiction, as $A \Varangle_{S} B$ and $B \Varangle_{S} C$.

Thus we conclude that $\leqslant_{S}$ is quasi-reflected.

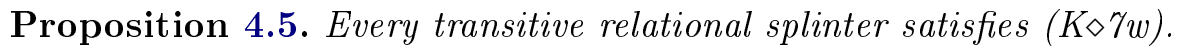

Proof. Let us suppose for contradiction that there is some transitive relational splinter $\diamond_{\delta}$ that does not satisfies $(\mathrm{K} \diamond 7 \mathrm{w})$. Thus, that for some formulae $\varphi$ and $\alpha, \delta(\varphi) \subset \omega(\alpha)$, but $\delta(\varphi) \nsubseteq \delta(\alpha)$ and there is $\psi^{\prime}$ that entails $\alpha$, such that $\delta\left(\psi^{\prime}\right) \cap \delta(\varphi) \neq \emptyset$.

Thus, there is a $A \in \delta(\varphi)$ such that $\varphi \notin \delta(\alpha)$. Thus for some formula $\psi \in \Gamma, A \notin \delta(\psi)$. This means that $A \leqslant B$ for some $B \in \omega(\psi)$. Let $S$ be the set of all theories $B$ in $\omega(\psi)$ such that $A \leqslant B$. As $\leqslant$ is transitive and all $C \in \delta(\psi)$ is in $\omega(\varphi)$ we get that $S \subseteq \omega(\psi) \backslash \omega(\varphi)$, since $A \in \delta(\varphi)$. This implies that there is a formula $\psi^{\prime}$ such that $S=\omega\left(\psi^{\prime}\right)$. This means that there is some $C \in \delta\left(\psi^{\prime}\right)$, that is, $C$ is maximal in $\omega\left(\psi^{\prime}\right)$ modulo $\leqslant$. Moreover, as $\omega(\alpha)=\bigcup_{\psi \in \Gamma} \omega(\psi)$, we get that for some $\psi \in \Gamma, C \in \omega(\psi)$. However, as $C \in \omega\left(\psi^{\prime}\right)=S$, we get that $C \notin \delta(\varphi)$ which means that there is some $B \in \omega(\psi)$ such that $(C \leqslant B$. Thus, as $\leqslant$ si transitive we get that $A \leqslant B$ which means that $A$ is not maximal in $\omega(\varphi)$ which is a contradiction since it belongs to $\delta(\varphi)$.

Lemma D.2. Let $\diamond_{\mu}$ be a mirrored royal splinter. Given a complete consistent theory $S$,

$$
\text { if } \neg \psi \notin K \diamond_{\mu} \varphi \text {, then } \mu(S, \varphi \wedge \psi) \subseteq \mu(S, \varphi) \cap \omega(\psi) \text {. }
$$

Proof. Let us suppose for contradiction that $\diamond_{\delta}$ does not satisfy $(\mathrm{K} \diamond 10)$. Therefore, for some complete consistent theory $K$ and formula $\psi$, we get that $\neg \psi \notin K \diamond_{\delta} \varphi$, but $\delta(\varphi \wedge \psi) \nsubseteq \varnothing \delta(\varphi) \cap \omega(\psi)$. Thus there is a $S \in \delta(\varphi \wedge \psi)$ such that $S \notin \delta(\varphi) \cap \omega(\psi)$. As $S \in \delta(\varphi \wedge \psi)$, we get that $S \in \omega(\varphi \wedge \psi)$ which means that $S \in \omega(\psi)$. Therefore, $S \notin \delta(\varphi)$. Thus, for some $A \in \omega(\varphi), S \leqslant_{K} A$. As $\neg \psi \notin K \diamond_{\delta} \varphi$, we get that for some $B \in \delta(\varphi), B \in \omega(\psi)$. Therefore, $B \in \omega(\varphi \wedge \psi)$. As $S \in \delta(\varphi \wedge \psi)$, we get that 
$S \Varangle_{K} B$, and since $B \in \delta(\varphi)$, we get that $B \Varangle_{K} A$ and $B \nless S$. However, as the relation $\leqslant_{K}$ is mirrored this implies that $B \leqslant A$ which is a contradiction.

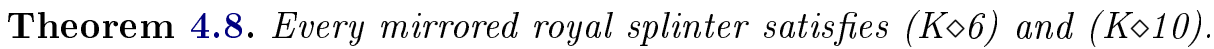

Proof. Let $\diamond_{\mu}$ be a mirrored royal splinter. As $\diamond_{\mu}$ is royal we have, from Theorem 4.4 that it satisfies $(\mathrm{K} \diamond 6)$. To show that it satisfies $(\mathrm{K} \diamond 10)$, let $S$ be a complete consistent theory, such that $\neg \psi \notin K \diamond_{\mu} \varphi$, we will show that $K \diamond_{\mu} \varphi+\psi \subseteq K \diamond_{\mu} \varphi \wedge \psi$. From Lemma D.2, we get that $\mu(K, \varphi \wedge \psi) \subseteq \mu(K, \varphi) \cap \omega(\psi)$.

As $K$ is complete, we get from Observation 4.2 that

$$
K \diamond_{\mu} \varphi=\bigcap \mu(K, \varphi)
$$

Therefore, $\mu(K, \varphi) \subseteq \omega\left(K \diamond_{\mu} \varphi\right)$. Thus,

$$
\mu(K, \varphi \wedge \psi) \subseteq \omega\left(K \diamond_{\mu} \varphi\right) \cap \omega(\psi) .
$$

Thus,

$$
\bigcap\left(\omega\left(K \diamond_{\mu} \varphi\right) \cap \omega(\psi)\right) \subseteq \bigcap \mu(K, \varphi \wedge \psi)
$$

This implies from Corollary D.3 that

$$
K \diamond_{\mu} \varphi+\psi \subseteq \bigcap \mu(K, \varphi \wedge \psi)
$$

Which from Observation 4.2 gives us

$$
K \diamond_{\mu} \varphi+\psi \subseteq K \diamond_{\mu} \varphi \wedge \psi
$$

Proposition D.2. Given a splinter $\diamond_{\delta}$, and a consistent complete theory K. If $\diamond_{\delta}$ satisfies both $(K \diamond 6)$ and $(K \diamond 8)$, then the projection of $\delta$ modulo $K$ is the silhouette of $\delta$ within $K$.

Proof. Let $K$ be a consistent complete theory, and $\leqslant_{K}$ the projection of $\delta$ modulo $K$. To show that $\leqslant_{K}$ is the silhouette of $\delta$ modulo $K$, we need to show that for every formula consistent formula $\varphi$

$$
\delta(K, \varphi)=\operatorname{Max}_{\leqslant_{K}}(\omega(\varphi)) .
$$

We need to show that (i) $\operatorname{Max}_{\leqslant_{K}}(\omega(\varphi)) \subseteq \delta(K, \varphi)$ (ii) $\delta(K, \varphi) \subseteq \operatorname{Max}_{\leqslant_{K}}(\omega(\varphi)$ ). Case (i) is trivial, since $S^{\prime} \leqslant_{K} S$ for every $S \in \delta(K, \varphi)$ and $S^{\prime} \in \omega(\varphi) \backslash \delta(S, \varphi)$. So we proceed to the case (ii).

(ii) Let us suppose for contradiction that $\delta(K, \varphi) \nsubseteq \operatorname{Max}_{\leqslant_{K}}(\omega(\varphi))$. Thus, there is some $S \in$ $\delta(K, \varphi)$ such $S \notin \operatorname{Max}_{\leqslant_{K}}(\omega(\varphi))$. Thus, $S \leqslant_{K} S^{\prime}$, for some $S^{\prime} \in \omega(\varphi)$. Thus, (a) $\left(S, S^{\prime}\right) \in \leqslant_{\varphi}$ or (b) $\left(S, S^{\prime}\right) \in \nabla(\varphi, \psi)$, for some formula $\psi$. 
(a) $\left(S, S^{\prime}\right) \in \leqslant_{\psi}$. This implies that $S \notin \delta(K, \varphi)$ which is a contradiction.

(b) $\left(S, S^{\prime}\right) \in \nabla(\varphi, \psi)$. Thus, $S^{\prime} \in \delta(\psi)$ and $S \in \omega(\varphi) \backslash(\delta(\varphi) \cup \omega(\varphi))$. This implies that $S \notin \delta(\varphi)$ which is a contradiction.

Thus, $\delta(K, \varphi) \subseteq \operatorname{Max}_{\leqslant_{K}}(\omega(\varphi))$.

Therefore, we conclude that $\delta(K, \varphi)=\operatorname{Max}_{\leqslant_{K}}(\omega(\varphi))$.

Theorem D.1. Every update function that satisfies $(K \diamond 6)$ and $(K \diamond 10)$ is a mirrored royal splinter.

Proof. Let $\diamond_{\delta}$ be an update function that satisfies both $(\mathrm{K} \diamond 6)$ and $(\mathrm{K} \diamond 10)$. From Proposition D.2, we have that every complete consistent theory $K$ the projection of $\delta$ modulo $K$ is the silhouette of $\delta$ within $K$. Therefore, analogously to the proof of Theorem 4.6, get that $\diamond_{\delta}$ is relational. Therefore, $\diamond_{\delta}$ is a royal splinter $\diamond_{\mu}$. We only need to show now that for every complete consistent theory $S$, the projection $\leqslant_{S}$ is mirrored. Let us suppose for contradiction that it is not mirrored. Then, for some complete consistent theories $A, B$ and $C$ :

$$
A \Varangle_{S} B, B \Varangle_{S} A, A \leqslant{ }_{S} C \text {, but } B \nless_{S} C
$$

As $A$ is a consistent complete theory, it follows that for some consistent formula $\varphi, A \in \omega(\varphi)$. This gives us two main cases to consider: (1) $B \in \omega(\varphi)$ or (2) $B \notin \omega(\varphi)$.

(1) $B \in \omega(\varphi)$. As $A \leqslant{ }_{S} C$, we get that: (a) the pair $(A, C)$ is either in $\leqslant_{\varphi}$ or (b) for some formula $\alpha,(A, C) \in \nabla(\varphi, \alpha)$ or $(A, C) \in \nabla(\alpha, \varphi)$.

(a) $(A, C) \in \leqslant{ }_{\varphi}$. This implies that $C \in \delta(\varphi)$, and $A \notin \delta(\varphi)$. Concerning $B$, we have that either $B \in \delta(\varphi)$, which implies that $A \leqslant_{\varphi} B$, or $B \notin \delta(\varphi)$, which leads to $B \leqslant_{\varphi} C$. In either case we reach a contradiction, as $A \Varangle_{S} B$ and $B \Varangle_{S} C$.

(b) $(A, C) \in \nabla(\varphi, \alpha)$ or $(A, C) \in \nabla(\alpha, \varphi)$. For the latter case, we have that $A \notin \omega(\varphi)$ which is a contradiction. So we focus only on the case $(A, C) \in \nabla(\varphi, \alpha)$. So $A \notin \delta(\varphi)$ and $C \in \delta(\alpha)$. Regarding $B$, we have that either $B \in \delta(\varphi)$, which implies that $A \leqslant{ }_{S} B$, or $B \notin \delta(\varphi)$ which implies that $B \leqslant_{S} C$. In either case we get a contradiction, as $A \Varangle_{S} B$ and $B{ }_{S} C$.

(2) $B \notin \omega(\varphi)$. Thus, for some formula $\psi, B \in \omega(\psi)$. Moreover, for some formula $\alpha$ : either (a) $(A, C) \in \leqslant_{\alpha}$ or $(\mathrm{b})(A, C) \in \nabla(\varphi, \alpha)$ or $(A, C) \in \nabla(\alpha, \varphi)$.

(a) $(A, C) \in \leqslant \alpha$. This means that $A \notin \delta(\alpha)$ and $C \in \delta(\alpha)$. Regarding $B$, we have two cases: (i) $B \in \omega(\alpha)$ or (ii) $B \notin \omega(\alpha)$.

(i) $B \in \omega(\alpha)$. Thus, either $B \in \delta(\alpha)$ which implies that $A \leqslant{ }_{S} B$, or $B \notin \delta(\alpha)$ which implies that $B \leqslant_{S} C$. In either case, we reach a contradiction, as $A \Varangle_{S} B$ and $B \mathbb{S}_{S} C$.

(ii) $B \notin \omega(\alpha)$. Thus, either $B \in \delta(\varphi)$, which implies from the triangulation $\nabla(\alpha, \psi)$ that $A \leqslant_{S} B$, or $B \notin \delta(\psi)$ which implies from the triangulation $\nabla(\psi, \alpha)$ that $B \leqslant_{S} C$. In ither case we get a contradiction, since $A \Varangle_{S} B$ and $B \$_{S} C$. 
(b) $(A, C) \in \nabla(\varphi, \alpha)$ or $(A, C) \in \nabla(\alpha, \varphi)$. For the latter case, we have that $A \notin \omega(\varphi)$ which is a contradiction. So we proceed to the case $(A, C) \in \nabla(\varphi, \alpha)$. Thus, $A \notin \delta(\varphi)$ and $C \in \delta(\alpha)$. Regarding $B$, we have that: either (i) $B \in \delta(\psi)$ or (ii) $B \notin \delta(\psi)$.

(i) $B \in \delta(\psi)$. Thus, from the triangulation $\nabla(\varphi, \psi)$ we have that $A \leqslant_{S} B$, which is a contradiction, since $A \Varangle_{S} B$.

(ii) $B \in \delta(\psi)$. In the case that $B \notin \omega(\alpha)$ we get from $\nabla(\psi, \alpha)$ that $B \leqslant{ }_{S} C$ which is a contradiction. On the other hand, if $B \in \omega(\alpha)$ we get that either $B \in \delta(\alpha)$, which implies that $A \leqslant_{S} B$, or $B \notin \delta(\alpha)$ which implies that $B \leqslant_{S} C$. In either case, we get a contradiction, as $A \Varangle_{S} B$ and $B \Varangle_{S} C$.

Thus we conclude that $\leqslant_{S}$ is mirrored. 


\section{Appendix E}

\section{Proofs for Chapter 5}

Theorem 5.2. If a belief change operator $*$ is AGM rational then its induced inference relation $\sim$ is a non-monotonic inference relation.

Proof. Let $K$ be a theory, * an AGM rational revision function, and $\sim$ the induced inference relation from $*$. Then, a formula $\psi \in K * \varphi$ iff $\varphi \sim \psi$.

N1. Let us suppose that $\psi \in C n(\varphi)$, then as $C n$ is Tarskian, we have $C n(\psi) \subseteq C n(\varphi)$. Moreover, from $\left(\mathbf{K}_{2}^{*}\right)$, we have that $\varphi \in K * \varphi$ which from the monotonicity of $C n$ implies that $C n(\varphi) \subseteq K * \varphi$. Therefore, $C n(\psi) \subseteq K * \varphi$ which means that $\psi \in K * \varphi$. This implies that $\varphi \sim \psi$.

N2. It is straightforward from $\left(\mathbf{K}_{6}^{*}\right)$.

N3. Let us suppose that $\varphi \sim \psi$ and $\alpha \in C n(\psi)$. This means that $\psi \in K * \varphi$. Then, as $C n$ is Tarskian, we have $C n(\alpha) \subseteq C n(\psi)$ and $C n(\psi) \subseteq K * \varphi$. Thus, $C n(\alpha) \subseteq K * \varphi$ which implies that $\alpha \in K * \varphi$. Therefore, $\varphi \sim \alpha$.

N4. Let us suppose that $\varphi \sim \psi$ and $\varphi \sim \alpha$. Then, $\psi, \alpha \in K * \varphi$. As $K * \varphi$ is a theory, this implies that $\psi \wedge \alpha \in K * \varphi$. Therefore, $\varphi \sim \psi \wedge \alpha$.

N5. Let us suppose that $\varphi \sim \psi$. Then, $\psi \in K * \varphi$. We have two cases to consider: $\varphi \in K$ or $\varphi \notin K$. We will show that on both cases $\varphi \rightarrow \psi \in K$.

- If $\varphi \in K$, then we have from $\left(\mathbf{K}_{3}^{*}\right)$ that $K * \varphi \subseteq K$. Then, as $\psi \in K * \varphi$, we have that $\psi \in K$ which implies that $\varphi \rightarrow \psi \in K$.

- If $\varphi \notin K$, then we have that either (i) $\neg \varphi \in K$ or (ii) $\varphi, \neg \varphi \notin K$. The case (i) is trivial that $\varphi \rightarrow \psi \in K$. For the case (ii), we have from $\left(\mathbf{K}_{3}^{*}\right)$ and $\left(\mathbf{K}_{4}^{*}\right)$ that $K * \varphi=K+\varphi$. Thus, as from hypothesis $\psi \in K * \varphi$, it follows from Observation 5.2 that $\varphi \rightarrow \psi \in K$.

So, we have that $\varphi \rightarrow \psi \in K$. As $K * \top=K$, this implies that $\varphi \rightarrow \psi \in K * \top$ which means that

$$
\sim \varphi \rightarrow \psi .
$$




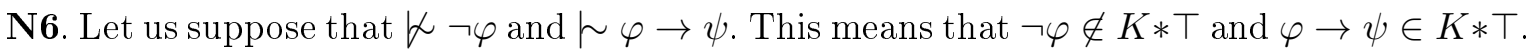
As $K * \top=K$, we have that

$$
\neg \varphi \notin K \text { and } \varphi \rightarrow \psi \in K .
$$

Then, it follows from $\left(\mathbf{K}_{4}^{*}\right)$ that $K+\varphi \subseteq K * \varphi$. Moreover, as $C n$ is Tarskian, we have that $K \subseteq K+\varphi$. Therefore, $K \subseteq K * \varphi$. Then, as $\varphi \rightarrow \psi \in K$, we have that

$$
\varphi \rightarrow \psi \in K * \varphi
$$

Thus, as from $\left(\mathbf{K}_{2}^{*}\right) \varphi \in K * \varphi$, we have that $\psi \in K * \varphi$, that is, $\varphi \sim \psi$.

N7. Its is straightforward from $\left(\mathbf{K}_{5}^{*}\right)$.

Theorem 5.3. Let $*$ be a belief revision operator that satisfies $\left(\boldsymbol{K}_{1}^{*}\right)$, and $\sim$ be its induced inference relation. Then,

$\boldsymbol{i}$ if $*$ satisfies $\left(\boldsymbol{K}_{7}^{*}\right)$, then $\sim$ satisfies $\mathbf{N 8}$;

ii if $*$ satisfies $\left(\boldsymbol{K}_{8}^{*}\right)$, then $\sim$ satisfies $\mathbf{N g}$.

Proof. Let $K$ be a theory and $\varphi$ and $\psi$ tow formulae. Let us suppose that $*$ satisfies $\left(\mathbf{K}_{1}^{*}\right)$.

i Let us suppose that $*$ satisfies $\left(\mathbf{K}_{7}^{*}\right)$, we will show that $\sim \sim$ satisfies $\mathbf{N 8}$. To show that, let us suppose that $\varphi \wedge \psi \sim \alpha$, thus we will have to show that $\varphi \sim \psi \rightarrow \alpha$. As $\sim \sim$ is induced from $*$, we have that $\alpha \in K * \varphi \wedge \psi$. Moreover, as by hypothesis $*$ satisfies $\left(\mathbf{K}_{7}^{*}\right)$, we have that

$$
K * \varphi \wedge \psi \subseteq K * \varphi+\psi
$$

which implies that $\alpha \in K * \varphi+\psi$. This together with Observation 5.2 implies that $\psi \rightarrow \alpha \in$ $K * \varphi$. Therefore, $\varphi \sim \psi \rightarrow \alpha$.

ii Let us suppose that $*$ satisfies $\left(\mathbf{K}_{8}^{*}\right)$, we will show that $\sim$ satisfies $\mathbf{N 9}$. To show that, let us suppose that $\varphi \not \alpha \neg \psi$ and $\varphi \sim \alpha$, thus we will show that $\varphi \wedge \psi \sim \alpha$.

As $\sim$ is induced from $*$, we have that $\psi \in K * \varphi$ iff $\varphi \sim \psi$. Thus, as by hypothesis $\varphi \not \downarrow \neg \psi$ and $\varphi \sim \alpha$, we have that $\neg \psi \notin K * \varphi$ and $\alpha \in K * \varphi$. As $*$ satisfies $\left(\mathbf{K}_{8}^{*}\right)$ and $\neg \psi \notin K * \varphi$, it follows that

$$
K * \varphi+\psi \subseteq K *(\varphi \wedge \psi)
$$

As $C n$ is Tarskian, we have that $K * \varphi \subseteq K * \varphi+\psi$. Therefore, as $\alpha \in K * \varphi$, we have $\alpha \in K * \varphi+\psi$ which from (E.1) implies that $\alpha \in K * \varphi \wedge \psi$. This means that $\varphi \wedge \psi \sim \alpha$.

Theorem 5.4. Let $\sim$ be the induced inference relation from the belief change function $*$ of Example 17 , then $\sim *$ is a non-monotonic inference relation. 
Proof. We have two main cases to consider: (i) $K * \varphi=K \otimes \varphi$ and (ii) $K * \varphi=(K+p)+\varphi$. As $\otimes$ satisfies all the basic AGM postulates, for the case that $K * \varphi=K \otimes \varphi$ it follows from Theorem 5.2 that $\sim$ satisfies the basic non-monotonic axioms. So we just need to focus on the case (ii) $K * \varphi=(K+p)+\varphi$. The axioms $\mathbf{N} 1$ and $\mathbf{N} 2$ are trivial. So we focus only on the another ones.

N3. Let us suppose that $\varphi \sim \psi$ and $\alpha \in C n(\psi)$. This means that $\psi \in K * \varphi$. Then, due to monotonicity, we have $C n(\alpha) \subseteq C n(\psi)$ and $C n(\psi) \subseteq K * \varphi$. Thus, $C n(\alpha) \subseteq K * \varphi$ which implies that $\alpha \in K * \varphi$. Therefore, $\varphi \sim \alpha$.

N4 Let us suppose that $\sim \psi$ and $\sim \alpha$. This means that $\psi \in K * \top$ and $\alpha \in K * \top$. Thus $\varphi \wedge \psi \in K * \top$, which implies that $\sim \psi \wedge \alpha$.

N5 Let us suppose that $\varphi \sim \psi$. Then, $\psi \in K * \varphi$. As we are considering the case $K * \varphi=$ $K+p+\varphi$, we have that $\psi \in K+p+\varphi$ which from Observation 5.2 implies that $\varphi \rightarrow \psi \in K+p$. As $K * \top=K+p$, we have that $\varphi \rightarrow \psi \in K * \top$. Thus, $\sim \varphi \rightarrow \psi$.

N6 Let us suppose that $\not \mathcal{\varphi} \neg$ and $\sim \varphi \rightarrow \psi$. This means that $\neg \varphi \notin K * \top$ and $\varphi \rightarrow \psi \in K * \top$. From definition of $*$, we have that $K * \top=K+p+\top$ which means that

$$
K * \top=C n(p)
$$

Moreover, we are considering that $K * \varphi=K+p+\varphi$, which implies:

$$
C n(p) \subseteq K * \varphi
$$

As by hypothesis $\varphi \rightarrow \psi \in K * \top$ and $K * \top=C n(p)$, we have then that $\varphi \rightarrow \psi \in C n(p)$. Thus, as $C n(p) \subseteq K * \varphi$, we have that $\varphi \rightarrow \psi \in K * \varphi$. Then, as as $\varphi \in K * \varphi$, it follows that $\psi \in K * \varphi$. Therefore, $\varphi \sim \psi$.

N7 This case is trivially satisfied, as for the case that $K * \varphi=K+p+\varphi, K * \varphi$ is always consistent. To see this, let us suppose for contradiction that $K * \varphi=K+p+\varphi$ and $\perp \in K * \varphi$. Thus, from definition of $*$, we have that

$$
\varphi \rightarrow \neg p \notin K
$$

Moreover, from $\perp \in K * \varphi$ and $K * \varphi=K+p+\varphi$ we have that $\perp \in K+p+\varphi$. Thus, from Observation 5.2 we get that $p \rightarrow \neg \varphi \in K$. As $p \rightarrow \neg \varphi$ is equivalent to $\varphi \rightarrow \neg p$, we get that $\varphi \rightarrow \neg p \in K$. This, however, contradicts (E.2). Therefore, for $K * \varphi=K+p+\varphi, \perp \notin K * \varphi$, that is, $\varphi \not \downarrow \perp$ which trivially satisfies $\mathbf{N} 7$.

Lemma E.1. If $\neg \psi \notin K+p+\varphi$, then $\neg \varphi \vee \neg \psi \vee \neg p \notin K$

Proof. Let us suppose for contradiction that $\neg \varphi \vee \neg \psi \vee \neg p \in K$, then $\varphi \rightarrow(\neg \psi \vee \neg p) \in K$. This implies that $\varphi \rightarrow(\neg \psi \vee \neg p) \in K+p+\varphi$. However, as $\varphi \in K+p+\varphi$ it follows that $\neg \psi \vee \neg p \in K+p+\varphi$. 
This means that $p \rightarrow \neg \psi \in K+p+\varphi$. Then, as $p \in K+p+\varphi$, we have that $\neg \psi \in K+p+\varphi$ which is a contradiction, as we assumed that $\neg \psi \notin K+p+\varphi$.

Observation 5.3. The belief change operator $*$ from Example 17 satisfies the supplementary AGM revision postulates.

Proof. We recall that the operator $\otimes$ on Example 17 is assumed to satisfy all the AGM revision postulates including the supplementary ones.

( $\left.\mathbf{K}_{7}^{*}\right)$ We need to show that $K * \varphi \wedge \psi \subseteq K * \varphi+\psi$. We have two cases to consider: (i) $(\varphi \wedge \psi) \rightarrow \neg p \in K$ or (ii) $(\varphi \wedge \psi) \rightarrow \neg p \notin K$

(i) $(\varphi \wedge \psi) \rightarrow \neg p \in K$, then $\neg \varphi \vee \neg \psi \in K$ which implies that $\varphi \wedge \psi \rightarrow \neg p \in K$. Thus,

$$
K * \varphi \wedge \psi=K \otimes \varphi \wedge \psi
$$

As $\otimes$ satisfies $\left(\mathbf{K}_{7}^{*}\right)$, we get that $K \otimes \varphi \wedge \psi \subseteq K \otimes \varphi+\psi$. This together with (E.3) implies

$$
K * \varphi \wedge \psi \subseteq K \otimes \varphi+\psi .
$$

We have two cases to consider: (a) $\varphi \rightarrow \neg p \in K$ or (b) $\varphi \rightarrow \neg p \notin K$.

(a) If $\varphi \rightarrow \neg p \in K$, then from definition of $*$ we have that $K * \varphi=K \otimes \varphi$ which from (E.4) implies that $K * \varphi \wedge \psi \subseteq K * \varphi+\psi$.

(b) If $\varphi \rightarrow \neg p \notin K$, then $K * \varphi=K+\varphi+p=K \otimes \varphi+p$. As $K \otimes \varphi \wedge \psi \subseteq K \otimes \varphi+\psi$ this implies that $K \otimes \varphi \wedge \psi \subseteq K \otimes \varphi+\psi+p$. Thus,

$$
K * \varphi \wedge \psi \subseteq K * \varphi+\psi
$$

Thus, from (a) and (b) we get thet $K * \varphi \wedge \psi \subseteq K * \varphi+\psi$.

(ii) $(\varphi \wedge \psi) \rightarrow \neg p \notin K$, then from definition of $*$, we have

$$
K * \varphi \wedge \psi=K+\varphi \wedge \psi+p
$$

As $(\varphi \wedge \psi) \rightarrow \neg p$ is equivalent to $\neg \varphi \vee \neg \psi \vee \neg p$ which is not in $K$, it follows that $\neg \varphi \vee \neg p \notin K$. This means that $\varphi \rightarrow \neg p \notin K$ which from the definition of $*$ implies that

$$
K * \varphi=K+\varphi+p
$$

As $K+\varphi \wedge \psi=K+\varphi+\psi$, it follows from (E.8) that

$$
\begin{aligned}
K * \varphi \wedge \psi & =K+\varphi+\psi+p \\
& =K+\varphi+p+\psi .
\end{aligned}
$$

This implies from (E.6) that

$$
K * \varphi \wedge \psi=K * \varphi+\psi
$$


Thus, $K * \varphi \wedge \psi \subseteq K * \varphi+\psi$.

$\left(\mathbf{K}_{8}^{*}\right)$ Suppose that $\neg \psi \notin K * \varphi$. Then, either (i) $\varphi \rightarrow \neg p \in K$ or (ii) $\varphi \rightarrow \neg p \notin K$

(i) $\varphi \rightarrow \neg p \in K$, then $\varphi \wedge \psi \rightarrow \neg p \in K$ which implies that $K * \varphi=K \otimes \varphi$ and $K * \varphi \wedge \psi=K \otimes \varphi \wedge \psi$. As $\otimes$ satisfies $\left(\mathbf{K}_{8}^{*}\right)$, it follows that $K \otimes \varphi+\psi \subseteq K \otimes \varphi \wedge \psi$. Thus, $K * \varphi+\psi \subseteq K *(\varphi \wedge \psi)$.

(ii) $\varphi \rightarrow \neg p \notin K$. This means that

$$
K * \varphi=K+\varphi+p
$$

and $\neg \varphi \vee \neg p \notin K$. As $\neg \psi \notin K * \varphi$, it follows that $\neg \psi \notin K+p+\varphi$. Thus, it follows from Lemma E. 1 that $\neg \varphi \vee \neg \psi \vee \neg p \notin K$. This means that $\varphi \wedge \psi \rightarrow \neg p \notin K$ which implies from the definition of $*$ that

$$
K * \varphi \wedge \psi=K+(\varphi \wedge \psi)+p
$$

As $K+\varphi \wedge \psi=K+\varphi+\psi$, it follows that

$$
K * \varphi \wedge \psi=K+\varphi+\psi+p
$$

Thus,

$$
K+\varphi+p+\psi \subseteq K+\varphi+\psi+p
$$

Thus, from (E.7) and (E.8) we get

$$
K * \varphi+\psi \subseteq K *(\varphi \wedge \psi)
$$

Theorem 5.5. There is a non-monotonic inference relation that satisfies the supplementary nonmonotonic axioms, but its induced belief change operator violates one of the basic AGM revision postulates.

Proof. Let us take the non-monotonic inference relation $\sim$ whose induced belief change operation is the belief revision operator $*$ from Example 17 . We have already shown that $*$ violates $\left(\mathbf{K}_{3}^{*}\right)$. We only need to show that $\sim$ satisfies N8 and N9. From Proposition 5.3, the operator $*$ satisfies the AGM supplementary postulates. This implies, from Theorem 5.3, that $\sim$ satisfies both $\mathbf{N} 8$ and N9.

Theorem 5.6. Let $\sim$ be a inference relation and $*$ its induced belief change operator thus

(i) if $\sim$ satisfies $\mathbf{N 1}$, then $*$ satisfies $\left(\boldsymbol{K}_{2}^{*}\right)$ (success);

(ii) if $\sim$ satisfies $\mathbf{N 2}$, then $*$ satisfies $\left(\boldsymbol{K}_{6}^{*}\right)$ (extensionality);

(iii) if $\sim$ satisfies both $\boldsymbol{N}^{\boldsymbol{\gamma}}$ and $\boldsymbol{N 1}$ then $*$ satisfies $\left(\boldsymbol{K}_{5}^{*}\right)$ (consistency). 
Proof. (i) Let us suppose that $\sim$ satisfies N1. Then, as $\varphi \in C n(\varphi)$, we have from $\mathbf{N} 1$ that $\varphi \mid \sim \varphi$ which implies that $\varphi \in K * \varphi$. Thus, $*$ satisfies $\left(\mathbf{K}_{2}^{*}\right)$.

(ii) Let us suppose that $\sim$ satisfies $\mathbf{N 2}$. Let $\varphi$ and $\psi$ be two formulae such that $C n(\varphi)=$ $C n(\psi)$, we need to show $K * \varphi=K * \psi$. Suppose that a formula $\alpha$ is in $K * \varphi$, thus $\varphi \sim \alpha$. As $\sim$ satisfies $\mathbf{N 2}$, we have that $\psi \sim \alpha$. Thus, $K * \varphi \subseteq K * \psi$. The side that $K * \psi \subseteq K * \varphi$ is analogous. Therefore, we conclude that $K * \varphi=K * \psi$.

(iii) ' $\Rightarrow$ ' Suppose that $K * \varphi=C n(\perp)$, then $\perp \in K * \varphi$ which implies that $\varphi \sim \perp$. Thus, from N7 $C n(\varphi)=C n(\perp)$, that is, $\neg \varphi \in C n(\emptyset)$.

' $\Leftarrow$ ' Suppose that $\neg \varphi \in C n(\emptyset)$, that is, $C n(\varphi)=C n(\perp)$. Thus, as $\varphi \in K * \varphi$ and $C n$ is Tarskian, we have that $C n(\varphi) \subseteq K * \varphi$. Then, as $C n(\varphi)=C n(\perp)$, it follows that $C n(\perp) \subseteq$ $K * \varphi$. However, $C n(\perp)$ contains all the formulae. Therefore, $K * \varphi=C n(\perp)$.

Proposition 5.1. An inference relation $\sim$ satisfies Closure $_{\mathrm{NM}}$ if and only if the belief change operator $*$ induced by it satisfies $\left(\boldsymbol{K}_{1}^{*}\right)$.

Proof. " $\Leftarrow$ " By the contrapositive, this is equivalent to show that if $\sim$ does not satisfy the closure then it does not satisfy $\left(\mathbf{K}_{1}^{*}\right)$. Let us suppose that $\sim$ does not satisfy the closure. Thus, for some formulae $\varphi$ and set of formulae $A$,

$$
\varphi \sim \psi, \text { for all } \psi \in A,
$$

and there is a formula $\alpha \in C n(A)$ such that $\varphi \not \alpha \alpha$. Thus,

$$
\alpha \notin K * \varphi
$$

From (E.9), we have that $A \subseteq K * \varphi$ which implies from the monotonicity of $C n$ that $C n(A) \subseteq$ $C n(K * \varphi)$. This implies that $\alpha \in C n(K * \varphi)$. Therefore, as $\alpha \notin K * \varphi$ and $\alpha \in C n(K * \varphi)$, we conclude that $K * \varphi$ is not a theory which violates $\left(\mathbf{K}_{1}^{*}\right)$.

" $\Rightarrow$ " Let $\sim$ be an inference relation that satisfies the closure. To show that its induced belief change operator $*$ satisfies $\left(\mathbf{K}_{1}^{*}\right)$, we need to show that each formula $\alpha \in C n(K * \varphi)$ is also in $K * \varphi$. Let us take a formula $\alpha \in C n(K * \varphi)$. As $*$ is induced from $\sim$, we have that a formula $\psi \in K * \varphi$ iff $\varphi \sim \psi$. Therefore, $\varphi \sim \psi$ for all formulae $\psi \in K * \varphi$. Thus, as $\alpha \in C n(K * \varphi)$, it follows from the closure that $\varphi \sim \alpha$. This implies that $\alpha \in K * \varphi$. Therefore, $*$ satisfies $\left(\mathbf{K}_{1}^{*}\right)$.

Proposition 5.2. The closure axioms implies N3 and N4.

Proof. Let $\sim$ be an inference relation that satisfies closure, and $A$ be a set of all formulae entailed from $\varphi$ by $\sim$, that is,

$$
A=\{\psi \in L \mid \varphi \sim \psi\}
$$


N3 Let us suppose that $\varphi \sim \psi$ and $\alpha \in C n(\psi)$. Thus, $\psi \in C n(A)$. Then, as $C n$ is Tarskian, we have that $C n(\psi) \subseteq C n(A)$. This implies that $\alpha \in C n(A)$. Therefore, from the closure, $\varphi \mid \sim \alpha$.

N4 Let us suppose that $\varphi \sim \psi$ and $\varphi \sim \alpha$. Thus, $\varphi \wedge \psi \in C n(A)$. Therefore, from the closure, $\varphi \sim \psi \wedge \alpha$.

Proposition 5.4. The belief change operation from Example 18 induces an inference relation $\sim$ which satisfies all basic axioms of non-monotonicity.

Proof. There are two cases to consider $K * \varphi=K \otimes \varphi$ or $K * \varphi=C n(\varphi)$. As $\otimes$ satisfies all the AGM postulates, for the case that $K * \varphi=K \otimes \varphi$, it follows from Theorem 5.2 that $\sim$ satisfies all the basic axioms of non-monotonicity. So we need to focus only on the case that

$$
K * \varphi=C n(\varphi)
$$

which from definition means that $\neg \varphi \notin K$. As $C n$ satisfies the deduction theorem, we have that

$$
\text { If } \psi \in C n(\varphi) \text {, then } \varphi \rightarrow \psi \in C n(\top) \text {. }
$$

The only two cases that deserve attention are N5 and N6, all the others are trivial.

N5 Let us suppose that $\varphi \mid \sim \psi$. Thus, $\psi \in K * \varphi$. Therefore, as $K * \varphi=C n(\varphi)$, we have that $\psi \in C n(\varphi)$. This implies from (E.10) that $\varphi \rightarrow \psi \in C n(\top)$, which means that $\varphi \rightarrow \psi \equiv \top$, therefore $\varphi \rightarrow \psi \in K * \top$. Thus, $\sim \varphi \rightarrow \psi$.

N6 Let $\not \mathcal{\neg} \varphi$ and $\sim \varphi \rightarrow \psi$. Thus, $\neg \varphi \notin K * \top$ and $\varphi \rightarrow \psi \in K * \top$. As $K * \top=C n(\top)$, it follows that $\varphi \rightarrow \psi \equiv \top$. Thus, as $K * \varphi$ is a theory, and all the tautologies are in every theory, we have that

$$
\varphi \rightarrow \psi \in K * \varphi
$$

Thus, as $\varphi \in K * \varphi$, we have that $\psi \in K * \varphi$ which implies that $\varphi \mid \sim \psi$. 
APPENDIX E 


\section{Bibliography}

Alchourrón, C. E. (1995). Defeasible logics: Demarcation and affinities. In Crocco, G., Del Cerro, L. F. n., and Herzig, A., editors, Conditionals: From philosophy to computer science, pages 67-102. Oxford University Press, Oxford, UK. 5, 6

Alchourrón, C. E., Gärdenfors, P., and Makinson, D. (1985). On the logic of theory change: partial meet contraction and revision functions. The Journal of Symbolic Logic, 50(2):510-530. 1, 12, $14,15,16,17,19,71,72$

Baader, F., Calvanese, D., McGuinness, D., Patel-Schneider, P., and Nardi, D. (2003). The description logic handbook: Theory, implementation and applications. Cambridge university press. 2, 21

Besnard, P. (2013). An introduction to default logic. Springer Science \& Business Media. 71

Bonanno, G. (2009). Belief revision in a temporal framework. New Perspectives on Games and Interaction, 4:45-80. 6

Cantwell, J. (2000). Logics of belief change without linearity. The Journal of Symbolic Logic, $65(4): 1556-1575.6$

Clarke, E. M., Grumberg, O., and Peled, D. A. (2001). Model checking. MIT Press. 7, 31

Darwiche, A. and Pearl, J. (1997). On the logic of iterated belief revision. Artificial Intelligence, $89(1): 1-29.6$

Dix, J. and Makinson, D. (1992). The relationship between KLM and MAK models for nonmonotonic inference operations. Journal of Logic, Language and Information, 1(2):131-140. 72

Flouris, G. (2006). On Belief Change and Ontology Evolution. PhD thesis, University of Crete. 8, $21,22,86$

Fuhrmann, A. (1991). Theory contraction through base contraction. J. Philosophical Logic, 20(2):175-203. 22

Gabbay, D., Rodrigues, O., and Russo, A. (2008). Belief revision in non-classical logics. The Review of Symbolic Logic, 1(3):267-304. 7

Gabbay, D. M., Hodkinson, I., and Reynolds, M. (1994). Temporal Logic (Vol. 1): Mathematical Foundations and Computational Aspects. Oxford University Press, Inc., New York, NY, USA. 2

Gärdenfors, P. (1988). Knowledge in flux: Modeling the dynamics of epistemic states. The MIT press. $1,5,6,12,18,19,71,86$

Gärdenfors, P. and Makinson, D. (1994). Nonmonotonic inference based on expectations. Artificial Intelligence, 65(2):197 - 245. 4, 36, 71, 72, 73

Ginsberg, M. L. (1986). Counterfactuals. Artif. Intell., 30(1):35-79. 36 
Grove, A. (1988). Two modellings for theory change. Journal of philosophical logic, 17(2):157-170. $6,19,20,42$

Guerra, P. d. T. (2016). Revisão de Crenças no Reparo de Especificações Formais de Sistemas. PhD thesis, Universidade de São Paulo. 2, 7, 25, 83

Guerra, P. T. and Wassermann, R. (2010). Revision of CTL models. In Advances in Artificial Intelligence - IBERAMIA 2010, 12th Ibero-American Conference on AI, Bahia Blanca, Argentina, November 1-5, 2010. Proceedings, pages 153-162. 7

Hansson, S. O. (1991). Belief contraction without recovery. Studia Logica, 50(2):251-260. 22

Hansson, S. O. (1999). A Textbook of Belief Dynamics: Theory Change and Database Updating, volume 11. Kluwer Academic. 1, 12, 22, 71

Heyting, A. (1956). Intuitionism an Introduction. North-Holland, Amsterdam. 21

Horn, A. (1951). On sentences which are true of direct unions of algebras. The Journal of Symbolic Logic, 16(1):14-21. 2, 21

Katsuno, H. and Mendelzon, A. O. (2003). On the difference between updating a knowledge base and revising it. In Gärdenfors, P., editor, Belief revision, pages 183-203. Cambridge University Press. $1,53,55,56,68$

Kraus, S., Lehmann, D., and Magidor, M. (1990). Nonmonotonic reasoning, preferential models and cumulative logics. Artificial intelligence, 44(1-2):167-207. 72

Lehmann, D. J. and Magidor, M. (1992). What does a conditional knowledge base entail? Artif. Intell., 55(1):1-60. 36

Lewis, D. (1976). Counterfactuals. Oxford. 36

Lindström, S. and Rabinowicz, W. (1991). Epistemic entrenchment with incomparabilities and relational belief revision. In The logic of theory change, pages 93-126. Springer. 6, 68

Makinson, D. and Gärdenfors, P. (1991). Relations between the logic of theory change and nonmonotonic logic. In The logic of theory change, pages 183-205. Springer. 71, 72

Meyer, T. A., Labuschagne, W. A., and Heidema, J. (2000). Refined epistemic entrenchment. Journal of Logic, Language and Information, 9(2):237-259. 36

Nayak, A. C. (1994). Iterated belief change based on epistemic entrenchment. Erkenntnis, 41(3):353390. 6

Nayak, A. C., Pagnucco, M., and Peppas, P. (2003). Dynamic belief revision operators. Artificial Intelligence, 146(2):193-228. 6

Nute, D. (1994). Handbook of Logic in Artificial Intelligence and Logic Programming. Oxford University Press. 71

Restall, G. and Slaney, J. (1995). Realistic belief revision. In Proceedings of the Second World Conference on Foundations of Artificial Intelligence (WOCFAI-95), pages 367-378. 2

Ribeiro, J. S., Nayak, A., and Wassermann, R. (2018). Towards belief contraction without compactness. In Principles of Knowledge Representation and Reasoning: Proceedings of the Sixteenth International Conference, KR 2018, pages 287-296. 25, 54

Ribeiro, J. S., Nayak, A., and Wassermann, R. (2019a). Belief change and non-monotonic reasoning sans compactness. In the Thirty-Third AAAI Conference on Artificial Intelligence, (forthcoming). 73 
Ribeiro, J. S., Nayak, A., and Wassermann, R. (2019b). Belief update without compactness in nonfinitary languages. In the Twenty-Eight International Joint Conference on Artificial Intelligence, (forthcoming). 54

Ribeiro, M. M. (2013). Belief revision in non-classical logics. Springer Science \& Business Media. $2,8,21,23$

Ribeiro, M. M., Wassermann, R., Flouris, G., and Antoniou, G. (2013). Minimal change: Relevance and recovery revisited. Artificial Intelligence, 201:59-80. 2, 22, 23, 25, 30, 83, 85

Rott, H. (1992). Preferential belief change using generalized epistemic entrenchment. Journal of Logic, Language and Information, 1(1):45-78. 1, 6, 68, 71

Van Ditmarsch, H., van Der Hoek, W., and Kooi, B. (2007). Dynamic epistemic logic, volume 337. Springer Science \& Business Media. 5

Wassermann, R. (2011). On AGM for non-classical logics. Journal of Philosophical Logic, 40(2):271294. 2

Winslett, M. (1988). Reasoning about action using a possible models approach. In Proceedings of the Seventh AAAI National Conference on Artificial Intelligence, AAAI'88, pages 89-93. AAAI Press. 53

Zhang, Y. and Ding, Y. (2008). CTL model update for system modifications. Journal of Artificial Intelligence Research, 31:113-155. 7 